Portland State University

PDXScholar

Winter 2-28-2013

\title{
Examining the Mechanisms of the Work-Nonwork Boundary Fit and Health Relationship
}

Jenna Risa LeComte-Hinely

Portland State University

Follow this and additional works at: https://pdxscholar.library.pdx.edu/open_access_etds

Part of the Industrial and Organizational Psychology Commons, and the Leisure Studies Commons Let us know how access to this document benefits you.

\section{Recommended Citation}

LeComte-Hinely, Jenna Risa, "Examining the Mechanisms of the Work-Nonwork Boundary Fit and Health Relationship" (2013). Dissertations and Theses. Paper 663.

https://doi.org/10.15760/etd.663

This Dissertation is brought to you for free and open access. It has been accepted for inclusion in Dissertations and Theses by an authorized administrator of PDXScholar. Please contact us if we can make this document more accessible: pdxscholar@pdx.edu. 
Examining the Mechanisms of the Work-Nonwork Boundary Fit and Health Relationship

by

Jenna Risa LeComte-Hinely

A dissertation submitted in partial fulfillment of the requirements for the degree of

\section{Doctor of Philosophy \\ in \\ Applied Psychology}
Dissertation Committee:
Leslie B. Hammer, Chair
Todd Bodner
Charlotte Fritz
Pamela Tierney
Liu-Qin Yang

Portland State University

2013 
C 2012 Jenna Risa LeComte-Hinely 


\begin{abstract}
This study examined the construct of work-nonwork boundary fit, or the congruence between an individual's work-nonwork boundary management preferences and the work-nonwork boundary management policies and practices supplied by their employer. The present study used boundary theory and person-environment (P-E) fit theory to propose that high levels of work-nonwork boundary fit would be beneficial to mental and physical health, both directly and indirectly via the dual mechanisms of conflict and enhancement. Survey methods and latent congruence modeling (LCM) were used to test these hypotheses, which were then supplemented by polynomial regression response surface mapping and qualitative analysis.

Results showed that high levels of boundary fit were beneficial for mental health over time, both directly and indirectly via lowered work-to-nonwork conflict. There was no support for the mechanism of work-nonwork enhancement, although this may be due to range restrictions within the data, such that most of the participants experienced very high levels of work-nonwork enhancement. Contrary to hypotheses, high levels of boundary fit was found to be detrimental for physical health over time. Potential reasons for these differential effects are proposed, as are contributions to the literature, practical applications, and directions for future research.
\end{abstract}




\section{Dedication}

This dissertation is dedicated to my husband, Braden Hinely, for giving me a good reason to segment work and nonwork domains. 


\section{Acknowledgements}

I would like to acknowledge my mentor, Dr. Jill Robinson, for inspiring me in this field, and for keeping my spirits up during graduate school. Without her, I would have never pursued this doctorate. I would like to acknowledge my committee members, Drs. Leslie Hammer, Todd Bodner, Charlotte Fritz, Pamela Tierney, and Liu-Qin Yang, for their critiques. This dissertation is immeasurably better due to their valuable advice. I would also like to acknowledge my cohort member, Gabriela Burlacu, for reviewing early drafts of this document, for giving me feedback, for helping me code the qualitative responses, and for supporting me during this process. I would also like to thank other members of my lab for their patience with me during the time it took me to design and implement this study, especially Chris Harper, Sarah Van Dyck, Tori Crain, Caitlin Demsky, and David Meier. I would also like to thank Juliana Slemenda, Jamie Taylor, Rachel Palmer, and Samantha Reiter-Akhavan for their support and for keeping me sane during the process. Mostly, I would like to acknowledge my family for their unending support, especially my parents, Dr. Juliet Brosing and Keith LeComte, and my husband, Braden Hinely. Without all of you, this doctorate would not have been possible. 


\section{Table of Contents}

Abstract ......................................................................................................................

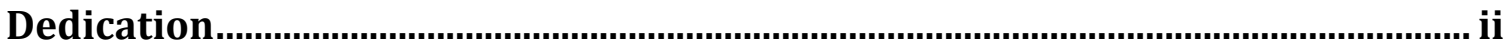

Acknowledgements ..................................................................................................

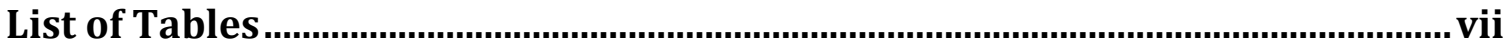

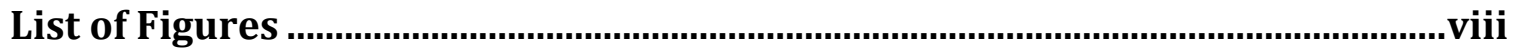

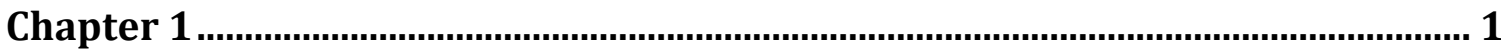

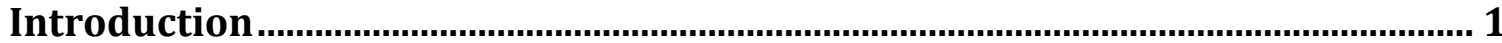

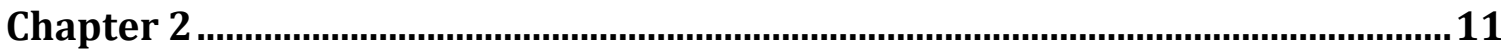

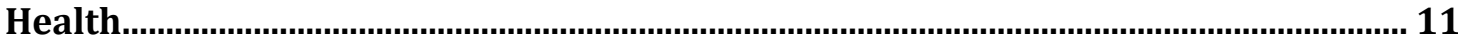

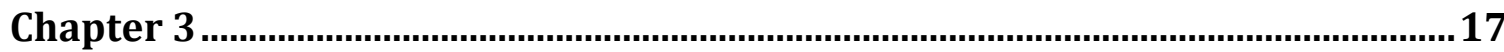

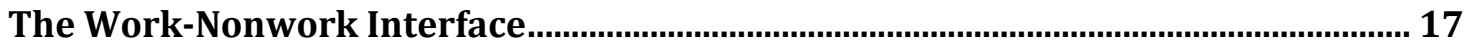

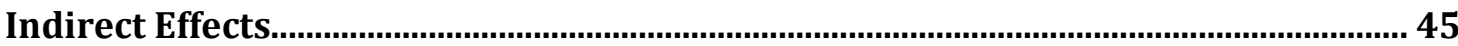

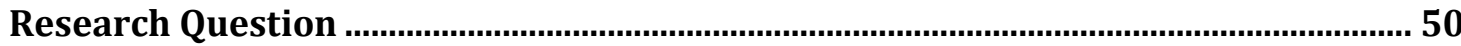

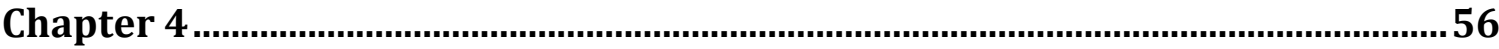

Methods ................................................................................................................. 56

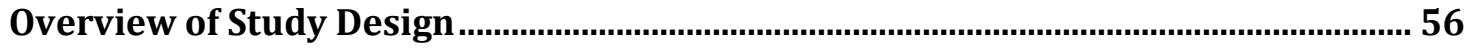

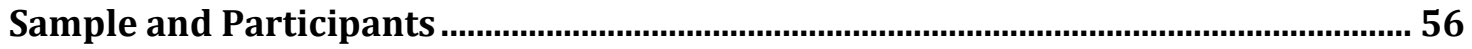

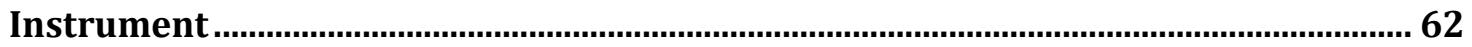

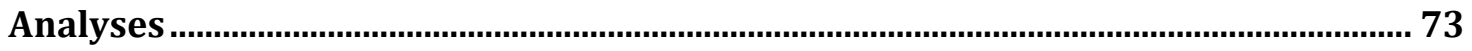


Chapter $5 \ldots \ldots \ldots \ldots \ldots \ldots \ldots \ldots$

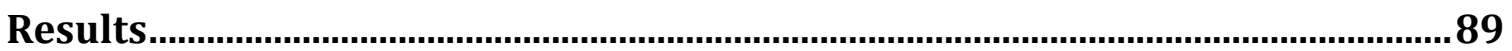

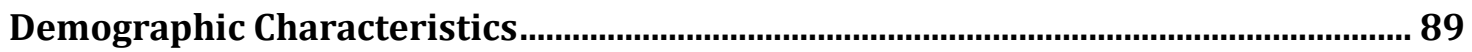

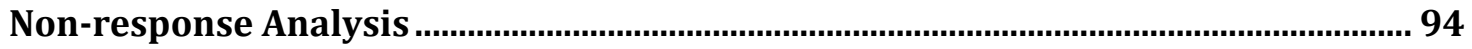

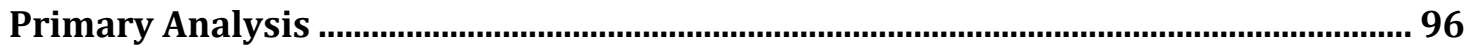

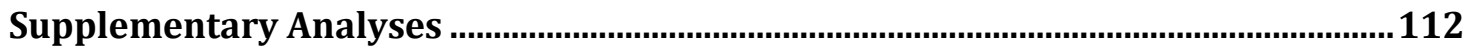

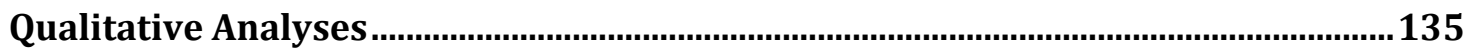

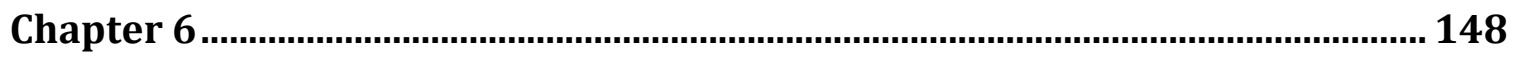

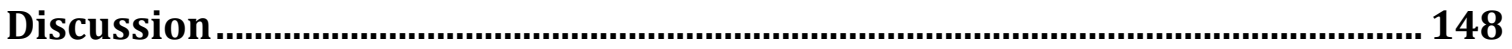

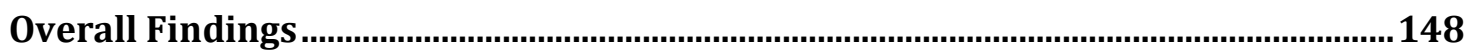

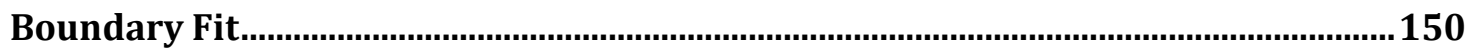

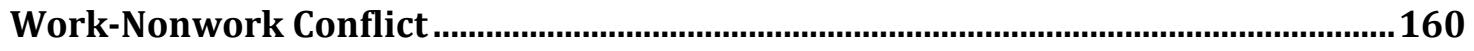

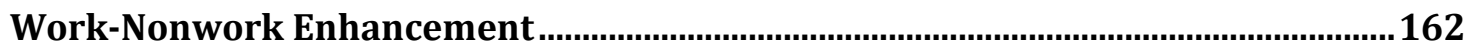

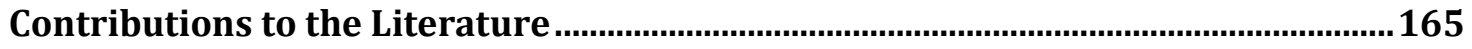

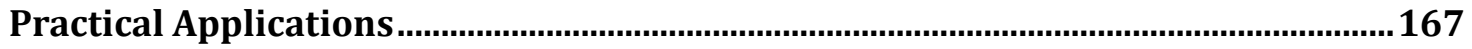

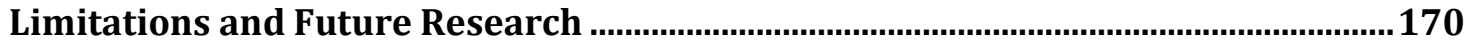

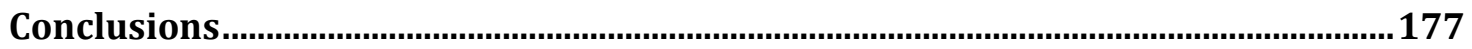

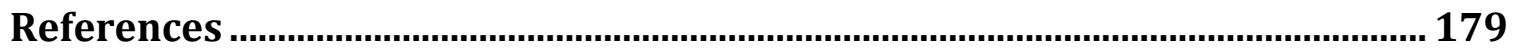

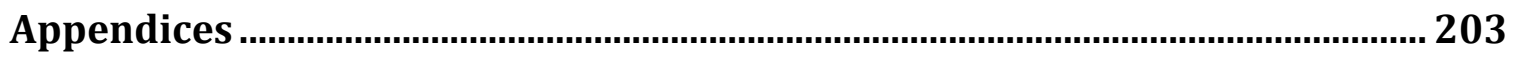

Appendix A: Time 1 Recruitment Materials................................................................. 204

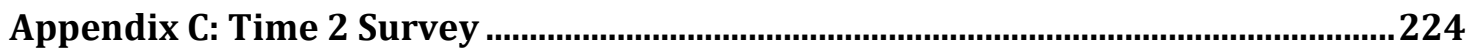

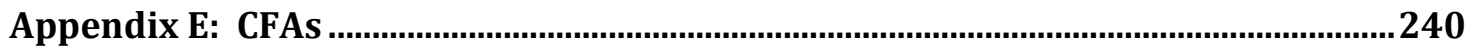




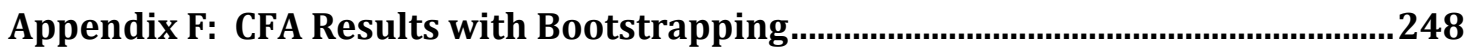

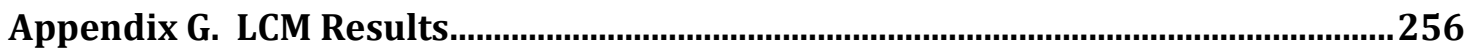

Appendix H: Qualitative Code List........................................................................

Appendix I: Simplified LCM without Mediator Results .................................................309

Appendix J: Significance Comparisons between Time 1 and Time 2 Mediator

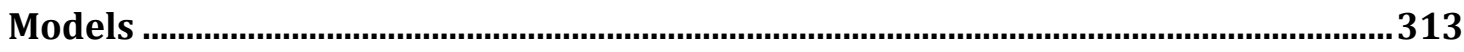

Appendix K. Supplementary Analyses for Non-Significant Polynomial Regression

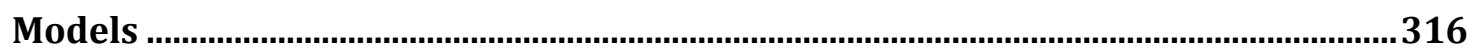




\section{List of Tables}

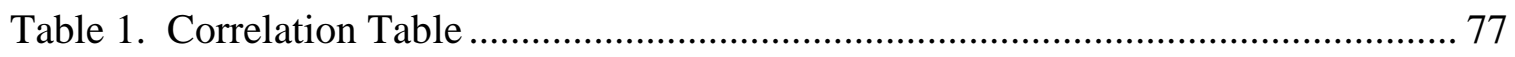

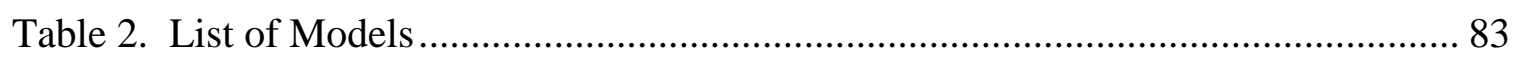

Table 3. Number of Open-Ended Responses ........................................................... 87

Table 4. Descriptive Statistics Regarding Income ..................................................... 90

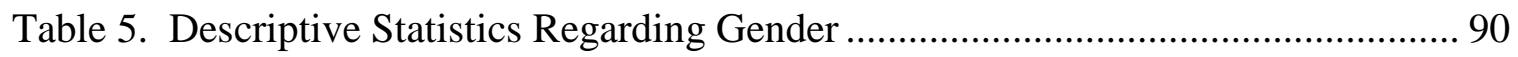

Table 6. Descriptive Statistics Regarding Education............................................ 91

Table 7. Descriptive Statistics Regarding Industry............................................... 91

Table 8. Descriptive Statistics Regarding Household Status at Time 1 ....................... 92

Table 9. Descriptive Statistics Regarding Household Status at Time 2 ....................... 92

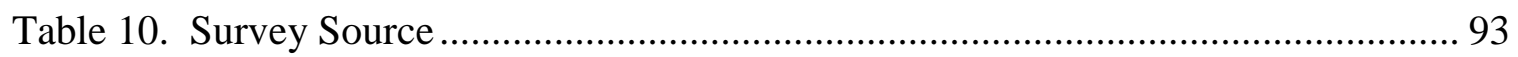

Table 11. Summary of Hypotheses and Results .................................................... 105

Table 12. Polynomial Regression Coefficient Significance Testing of Boundary Fit on

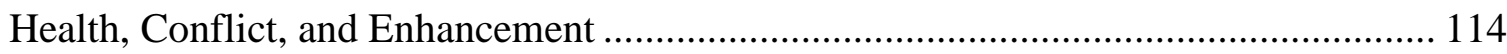




\section{List of Figures}

Figure 1. A graphic representation of the proposed model.......................................... 4

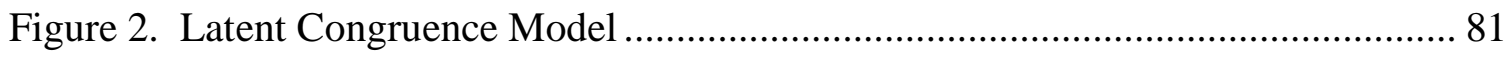

Figure 3. Simplified LCM without Mediators .......................................................... 111

Figure 4. Response Surface Map of Congruence \& Mental Health ............................. 116

Figure 5. $P=S$ and $P=-S$ Slopes for Congruence \& Mental Health ........................ 116

Figure 6. Response Surface Map of Congruence \& Physical Health ............................ 119

Figure 7. $P=S$ and $P=-S$ Slopes for Congruence \& Physical Health....................... 119

Figure 8. Response Surface Map of Congruence \& Work-to-Nonwork Conflict .......... 122

Figure 9. $P=S$ and $P=-S$ Slopes for Congruence $\&$ Work-to-Nonwork Conflict...... 123

Figure 10. Response Surface Map of Congruence \& Work-to-Nonwork Behavior-Based

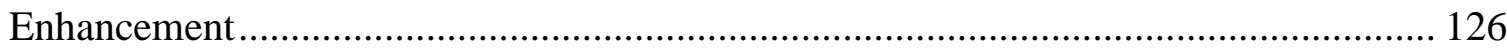

Figure 11. $P=S$ and $P=-S$ Slopes for Congruence \& Work-to-Nonwork Behavior-

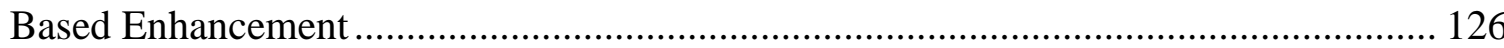

Figure 12. Response Surface Map of Congruence \& Work-to-Nonwork Value-Based

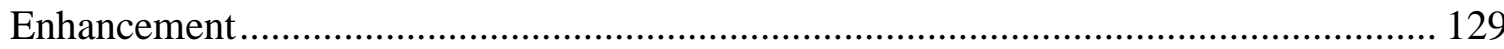

Figure 13. $P=S$ and $P=-S$ Slopes for Congruence \& Work-to-Nonwork Value-Based

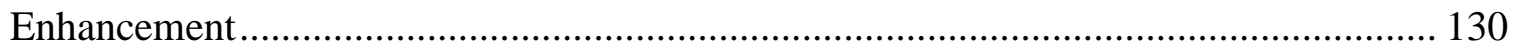

Figure 14. Response Surface Map of Congruence \& Nonwork-to-Work Value-Based

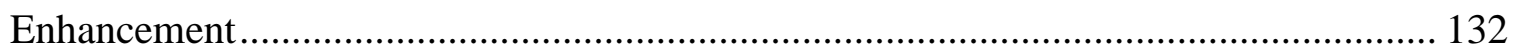

Figure 15. $P=S$ and $P=-S$ Slopes for Congruence $\&$ Nonwork-to-Work Value-Based

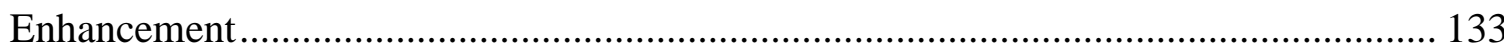




\section{Chapter 1}

\section{Introduction}

In 2010, nearly 4 million nonfatal workplace injuries and illnesses were reported in the U.S. (US Department of Labor, Bureau of Labor Statistics, 2011). More than onehalf of these reported illness and injury cases required days away from work, job transfer, or subsequent job restriction cases (U.S. Department of Labor, 2011). Even more concerning, recent research shows strong evidence that such estimates provided by national data systems such as the Bureau of Labor Statistics may be underestimating the number of workplace injuries and illnesses by more than two-thirds due to underreporting (Probst \& Estrada, 2010). For example, Rosenman, Kalush, Reilly, Gardiner, Reeves and Luo (2006) estimated that $68 \%$ of workplace accidents and injuries are not captured by the Occupational Safety and Health Administration (OSHA) or the BLS, while Probst, Brubaker, and Barsotti (2008) found that a disturbing 78\% of all actual accidents were unreported. This indicates that, realistically speaking, an estimated 12 million workers become injured or ill as a result of their work in a single year.

These statistics should be concerning to workers and organizations alike. Unhealthy workers, those suffering from occupational illness or injury, cannot function fully in their jobs, in their nonwork lives, nor in society as a whole. The absence of illness and disease, as well as the presence of positive states such as happiness and satisfaction, are necessary for individuals to make and reach goals, meet needs, and successfully cope with everyday life (Raphael et al., 1999). Healthy workers are better for the organization - they have higher productivity, lower absenteeism, and lower 
worker's compensation claims, to name a few desirable outcomes (Goetzel, Guindon, Turshen, \& Ozminkowski, 2001). Organizations with stellar employees like that can be much more successful than organizations with sick, exhausted, or absent employees. In order to be fully functioning, a society must have both successful individuals and successful organizations_-and this means promoting health.

How can we protect and promote worker health? What characteristics or aspects can organizations influence to encourage and improve the health of their workers?

Previous research indicates that it is possible for organizations to influence the health of their workers by providing a workplace that balances well with employee home life. In today's world, nearly every individual holds multiple roles in their life. These roles can be work-related roles, such as the role of a supervisor or a line worker, or nonworkrelated roles, such as the role of parent, spouse, religious leader or friend (Kahn, Wolfe, Quinn, Snoek, \& Rosenthal, 1964). It is inevitable that individuals who hold multiple roles may experience overlap between the roles, and that characteristics of the interface between domains can have an influence on outcomes such as health. This dissertation seeks to further investigate how to design the work-nonwork interface to promote and protect worker health.

For some workers, the ideal situation may be one where work and nonwork roles are completely segmented, with strict boundaries in place between the two domains. Conversely, other workers may function best when they can integrate their work and nonwork roles, answering calls from family members at work, and taking work emails after dinner at their home. Similarly, organizations may differ in terms of how much they 
allow or encourage their employees to segment or integrate their work and nonwork roles by allowing workers flexibility, control, and autonomy. For instance, one organization may expect workers to be available for consultation at home, or even oncall, while another organization may support workers who want to be completely "off the grid" when they are away from work (Ashforth, Kreiner, \& Fugate, 2000).

Previous research indicates that the degree of fit between how the individual prefers to balance their work and nonwork roles and how their organization allows or encourages that balance can significantly predict health (Edwards \& Rothbard, 1999). It appears that when organizations allow employees to manage their work and nonwork roles according to their specific preferences, the health of the employee is positively influenced.

Health also appears to be influenced by other aspects of the work-nonwork interface. For example, work-nonwork conflict, a form of interrole conflict that occurs when work and nonwork domains are mutually incompatible, has been shown to be damaging to the health of an individual (e.g., Allen, Herst, Bruck, \& Sutton, 2000; Kossek \& Ozeki, 1999; Mesmer-Magnus \& Viswesvaran, 2005; Van Steenbergen \& Ellemers, 2009). Conversely, work-nonwork enhancement, which occurs when positive affect, skills, behaviors, or values cross over between roles, has been shown to be beneficial for individual health (e.g., Allis \& O’Driscoll, 2008; Grzywacz \& Bass, 2003; Hanson, Hammer, \& Colton, 2006; Van Steenbergen \& Ellemers, 2009).

Despite these promising aspects of the work-nonwork interface that influence health, it appears that no single study has yet proposed how these three constructs may 
relate to each other and to health. In this dissertation, I built on theory and empirical research on the constructs of work-nonwork boundary fit, work-nonwork conflict, and work-nonwork enhancement to construct a single model that depicts how these three constructs can influence (and be used to improve) health over time. See Figure 1 for a visual representation of the proposed model. In developing and testing this model in a diverse sample of participants, I hope to shed more light on what organizations can do to help their employees successfully balance work and nonwork roles and subsequently experience better health, thus improving both individual lives and the organizational bottom line.

Figure 1. A graphic representation of the proposed model

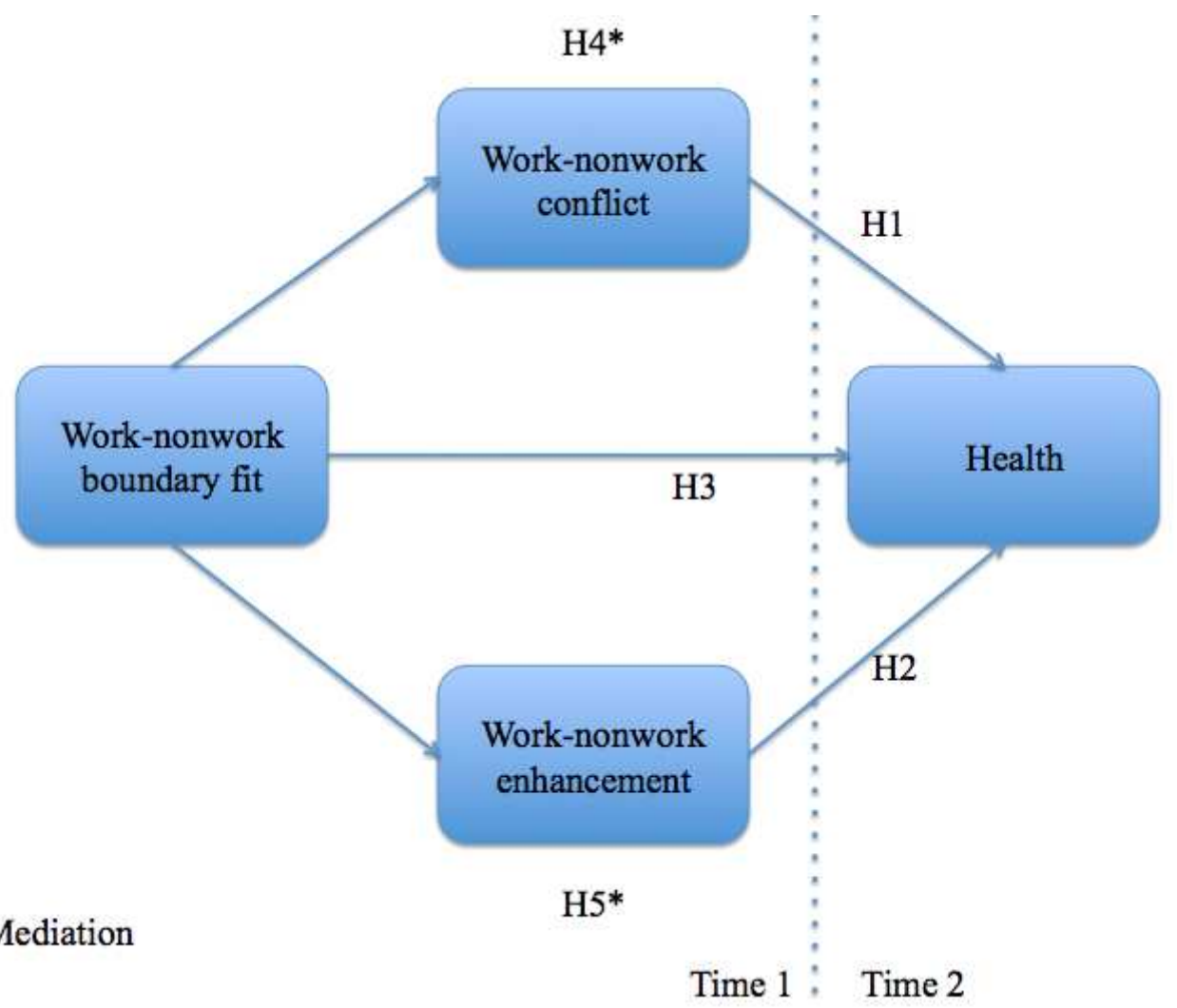


This dissertation offers several unique contributions to the occupational health psychology (OHP) field, including 1) creation of a single, concise model that includes work-nonwork boundary fit, work-nonwork conflict, work-nonwork enhancement, and two types of health, 2) design that includes multiple data collections to enable stronger causal inferences, 3) relatively rare application of P-E fit theory to work-nonwork research, 4) use of broad constructs to examine these phenomena in individuals without traditional families, thereby enabling better understanding of the work-nonwork interface for audiences traditionally underserved in I/O psychology, 5) cutting-edge method of analysis that allows more precise analysis of congruence issues, and 6) unusual hybrid study design that allows participants to express their experience both quantitatively and in their own words.

First, to my knowledge, previous research has only examined single parts of this proposed model, such as the link between work-nonwork boundary fit and work-nonwork conflict, or the link between conflict and health. However, my review of the literature indicates that no research yet exists that incorporates all of these findings into a single model, as is the case in this dissertation. To my knowledge, only one prior study examined boundary fit and health (Edwards \& Rothbard, 1999), and this study did not differentiate between the different types of health to assess potential subtleties of the relationship. The measures included in the current study allow for an examination of the differential effects of boundary fit on physical and mental health. Additionally, the majority of previous research on this topic (e.g., Chen, Powell, \& Greenhaus, 2009; Edwards \& Rothbard, 1999) has examined these phenomena cross-sectionally. To 
address this shortcoming, this study examined the constructs at two time points. While this does not completely enable causal inferences, it does create greater confidence in directionality of relationships. The literature in the field that did examine these phenomena over time (i.e., Kreiner, 2006) did not assess all measures at both time points, thus hindering the ability to examine questions of reverse causality. To address this, the current study includes all measures at all time points in order to give greater certainty to causal inferences by examining phenomena over time and allowing statistical study of potential reverse causation.

This dissertation was also designed to answer the call to apply personenvironment (P-E) fit literature to work-nonwork research. P-E fit is an excellent lens that can aid in our understanding of work-nonwork phenomena that has been, for the most part, neglected in the work-nonwork field (Chen et al., 2009; for exceptions, see Kreiner, 2006; Rothbard et al., 2005). The use of this theoretical lens to frame the current study contributes new knowledge to both the P-E fit field and the work-nonwork field, as well as providing an example of how the two fields can intersect for future research.

An additional contribution of this dissertation is to the broader field of worknonwork research. For many years, this research has focused strictly on work and family rather than the broader concept of nonwork or personal life. This restriction of the construct has resulted in work-family research focusing especially on married or partnered individuals with children living at home (Rothausen, 1999). While this research is helpful, as many of these workers do experience high levels of work-family conflict, it has restricted our broader knowledge, limited the generalizability of our 
research, and excluded examination of many populations of workers who may experience the work-nonwork interface much differently than married individuals with children. Several experts in the field have called for researchers to take a more inclusive stance on work-nonwork research and broaden the population included in their research (Parasuraman \& Greenhaus, 2002; Rothausen, 1999). By examining the constructs of work-nonwork conflict and work-nonwork enhancement (rather than the more restrictive constructs of work-family conflict and work-family enhancement) and by recruiting a sample diverse in nonwork roles and responsibilities, the results of this dissertation allow findings to be generalized to workers with many types of nonwork responsibilities, not just those with children at home.

Another unique contribution of this study is the method of analysis. I utilized latent congruence modeling (LCM), a new adaptation of structural equation modeling (SEM) designed to examine congruence issues in organizational research (Chen et al., 2009; Cheung, 2009). LCM has shown great promise in applications that examine the congruence between work-nonwork boundary preferences and workplace boundary policies and procedures (Chen et al., 2009). The statistical technique offers improvements over past techniques by allowing examination of individual components' impact on the outcome while simultaneously estimating and controlling for measurement error (Cheung, 2009). By using LCM, this study advances the field of congruence research by utilizing the best and most recent methodology to examine relationships, and advances the measurement field by lending further support to a relatively new technique. 
The survey instrument used in this study offered participants the opportunity to comment on these phenomena in the form of open-ended questions. This data was analyzed qualitatively, and allows greater depth of understanding of the quantitative findings. This hybrid study design is relatively unusual in I/O psychology, and allows a greater depth of insight to the theorized relationships between work-nonwork boundary fit, work-nonwork conflict, work-nonwork enhancement, and health (Lee, Mitchell, \& Harman, 2011).

This study makes several contributions to the literature. By including multiple types of health and multiple potential mediators, these results shed light on the complex relationship between work-nonwork boundary congruence, conflict, enhancement, and health and the differential effects of boundary fit on various outcomes. New directions for future research, built upon the current findings and theory, are proposed as a way to move the field forward.

This dissertation is organized as follows: in Chapter Two, the outcome of interest, health, is defined in order to give readers a deeper understanding of the complexity of this construct. To emphasize the importance of health, outcomes of poor health are described for individuals, organizations, and societies. To emphasize what can be done to influence health and thereby avoid negative consequences, predictors of health are reviewed. While health can be influenced by many factors, this dissertation focused on those factors within the realm of the work-nonwork interface. Thus, the work-nonwork interface is described next in Chapter Three, including three key constructs: work-nonwork conflict, work-nonwork enhancement, and work-nonwork boundary fit. First, work-nonwork 
conflict is defined, including relevant theory and previous research in relation to health, which leads to the proposal of Hypothesis 1. Next, work-nonwork enhancement is defined, including relevant theory and previous research in relation to health, which leads to the proposal of Hypothesis 2. Finally, work-nonwork boundary fit is defined, including relevant theory and previous research in relation to health, which leads to the proposal of Hypothesis 3. Subsequently, a rationale for indirect effects is proposed, leading to the proposal of Hypotheses 4 and 5. Finally, as an ancillary point, worknonwork boundary segmentation level is briefly discussed, leading to the proposal of a research question.

Chapter Four, the methods section, describes the recruitment in depth, and then moves on to the instrument, including each of the measures and their reliability. Next, the survey procedure is laid out in detail. Finally, the rationale for the choice of analysis technique is described, followed by an in-depth discussion of the three methods of analysis.

Chapter Five, the results section, begins with a demographic profile of the respondents, followed by primary analysis results, which are broken out by hypothesis. Additional analyses are discussed, followed by the secondary analysis results, and finally, the qualitative analysis results.

Finally, Chapter Six, the discussion section, begins with a summary of the main significant findings. Potential explanations are put forth to explain results that were not as hypothesized. Contributions to the literature and practical recommendations are 
discussed. Future directions for research are proposed, drawing from the limitations of the current study and the potential new avenues presented in the qualitative analyses. 


\section{Chapter 2}

\section{Health}

In order to adequately study the concept of employee health, we must first understand what health is and why organizations should be concerned with the health of employees. Health is made up of both physical and psychological attributes (e.g., Greenhaus, Allen, \& Spector, 2006). These can include physical health symptoms, overall health problems, emotions, satisfactions, dissatisfactions, mental disorders and more (Greenhaus et al., 2006). The term "health" generally includes both physiological and psychological symptomology in a medical context. In contrast, "well-being" is a broader construct that encompasses health but also includes context-free measures of life experiences (Danna \& Griffin, 1999). However, this particular study focuses on the health aspects within the broader construct of well-being, and thus only the term "health" is described in depth here.

Health has been conceptualized as the ability to make and reach goals, meet needs, and successfully cope with everyday life (Raphael et al., 1999). Positive health consists not only of a lack of negative conditions and diseases, but also a presence of positive states and conditions (Nelson \& Simmons, 2003). In other words, the absence of physical and mental complaints does not automatically create positive health in an individual.

Outcomes of health. It is no secret that positive health is a desirable state for individuals. Being free from physical and mental illnesses, having high levels of positive emotions and satisfactions, and low levels of dissatisfaction are all desirable outcomes 
(Nelson \& Simmons, 2003). Healthy individuals are able to make and reach goals, meet needs, and successfully cope with everyday life (Raphael et al., 1999). Individuals suffering from poor health are unable to fully function in any of their roles, be it work or nonwork-related. Healthy people are capable of enjoying a vigorous, satisfying life where they are able to contribute to society and to their own pastimes (Straub, 2007). Healthy individuals have an increased life expectancy, and more of those years are likely to be high quality life (U.S. Department of Health and Human Services, 2011). Positive health lays the foundation for success in all arenas of life. Thus, it is clear that it is in the best interests of individuals and society as a whole to have healthy, happy employees.

Promoting and protecting employee health is in the best interests of the organization as well. It is difficult to accurately capture the costs of poor employee health in organizations, simply due to the large number of factors that play into the equation. An unhealthy employee is expensive for an organization due to higher rates of absenteeism, lowered productivity while at work, higher health insurance costs, higher workers' compensation premiums, and potential legal costs (Goetzel et al., 2001). For example, individuals who suffer from psychological distress (anxiety and depression) are absent twice as often as those who are not distressed (Hardy, Woods, \& Wall, 2003). Absenteeism can be not only inconvenient, but also costly. Research estimates that costs associated with employee absenteeism and reduced productivity due to poor health are two to three times greater than the costs of medical and pharmacy claims (Edington \& Burton, 2003; Loeppke \& Hymel, 2006, Loeppke \& Hymel, 2008). One meta-analysis examining strain and absenteeism found that the annual losses due to absenteeism 
resulting from work strain cost between $\$ 17,400$ for small companies and $\$ 1.13$ million for larger companies, even without accounting for indirect costs such as health insurance claims or lost productivity (Darr \& Johns, 2008).

If all of these costs are accounted for, what is the total cost of poor employee health for organizations? One benchmarking study of nearly one million participants in 43 American companies determined that the median organizational loss due to employee health problems was $\$ 9,992$ per employee (Goetzel et al., 2001).

Other research has made an effort to determine the costs of individual illnesses for an organization. For example, Goetzel, Long, Ozminkowski, Hawkins, Wang, and Lynch (2004) studied the cost of poor health, based on absence, short-term disability, and productivity losses, for 10 major health conditions. Based on average impairment and prevalence estimates, their results showed that most health conditions such as hypertension, heart disease, depression, and arthritis cost the organization an average of anywhere between $\$ 300$ to $\$ 400$ per eligible employee per year (Goetzel et al., 2004). Similarly, another study found that asthma, high blood pressure, smoking, and obesity each reduce annual productivity by $\$ 200$ to $\$ 400$ per person (Mitchell \& Bates, 2011). Another study examining sleep patterns found that individuals suffering from insomnia and insufficient sleep syndrome had significantly worse productivity, and estimated that these productivity losses cost the organization approximately $\$ 2,000$ per employee per year (Rosekind, Gregory, Mallis, Brandt, Seal, \& Lerner, 2010). Depression alone is estimated to cost employers across the U.S. $\$ 44$ billion per year in lost worker productivity (Stewart, Ricci, Chee, Hahn, \& Morganstein, 2003). This enormous cost is 
likely due to the fact that depressed employees have an average productivity loss of more than $10 \%$ due to absenteeism and an additional $15 \%$ due to lack of presenteeism (Goetzel et al., 2004).

Thus, it is apparent that it is in the best interests of the organization to foster employee health, as it is beneficial for the individual, the organization, and society. The question then becomes, what can organizations do to promote and enhance the health of employees, thus protecting their workforce and their bottom line?

The answer to this question lies in a deeper understanding of the predictors of health. Once controllable predictors of health have been identified and understood, organizations can begin to alter them in ways that protect the health of employees, and through this, the profit margins of the organization. For example, one meta-analysis found that absenteeism costs were reduced by approximately $\$ 2.73$ and medical costs were reduced by approximately $\$ 3.27$ for every dollar spent on workplace wellness programs (Baicker, Cutler, \& Song, 2010). Thus, it is critical to study predictors of health that can be altered in the workplace if we are to make a difference for employees and organizations.

Predictors of health. Health is influenced by a vast multitude of factors, stemming from individual differences, genetics, behaviors, and environmental characteristics. Some of these factors, such as genetic predispositions or individual personality traits, are relatively invariable. Given this study focused on primary prevention of poor health and promotion of positive health states, these relatively unalterable factors are not the focus of this literature review. Instead, in keeping with the 
tenets of occupational health psychology (OHP), this literature review will focus on those predictors that are controllable and primarily under the control of the organization.

Even with these limitations, there are many aspects of the workplace that can have an impact on the health of workers. For instance, the simple logistics of work can have an influence on employee health, such as when and where work is done. Working nonstandard hours is detrimental to health (e.g., Smith, Folkard, \& Fuller, 2003), as is working in dangerous environments that can cause occupational illnesses or injuries (e.g., Barling \& Frone, 2004; Zohar, 2003). Having too much work to do can also negatively impact health by resulting in high levels of stress and fatigue (Grech, Neal, Yeo, Humphreys, \& Smith, 2009).

Characteristics of the work itself can also influence health. For example, decision latitude is a characteristic of work that allows employees authority over how their knowledge is utilized. Individuals who lose decision latitude or have chronically low decision latitude can suffer from high levels of cardiovascular disease (CVD), hypertension, and atherosclerosis (Landsbergis, Schnall, Pickering, Warren, \& Schwartz, 2003).

Who work is done with can also influence health. For example, employees who have supportive, effective leaders have lower levels of perceived stress, job strain, burnout, and depression (Kelloway \& Barling, 2010). In contrast, employees with abusive leaders who engage in sustained displays of hostile behaviors (Tepper, Henle, Lambert, Gacalone, \& Duffy, 2008) can experience negative health outcomes such as higher levels of burnout, feelings of helplessness, diminished self-efficacy, lowered 
organizational commitment, and higher levels of strain (Kelloway \& Barling, 2010).

Leaders aren't the only individuals at work who can impact the health of others.

Individuals who experience incivility, low-deviant behavior in violation of workplace norms for mutual respect, can have chronic feelings of discomfort, distress, and emotional exhaustion that can increase instances of blood pressure and heart disease (Lim, Cortina, \& Magley, 2008; Van Jaarsveld, Walker, \& Skarlicki, 2010). This incivility can be damaging whether it comes from supervisors, coworkers, or even customers (Van Jaarsveld et al., 2008).

Even a brief perusal of the literature makes it clear that there are many workplace factors that can influence an individual's health. In this study, I chose to focus on a set of predictors of health that stem not just from the workplace, but from the intersection of work and other life domains. I chose to focus on this complex set of predictors based upon their wide applicability to a variety of workers, the strong theories backing existing research, and the opportunity to explore under-developed areas of OHP. 


\section{Chapter 3}

\section{The Work-Nonwork Interface}

Role theory states that the majority of everyday actions are created by socially defined categories that are accumulated in an individual's life, such as supervisor, employee, spouse, parent, church leader, and more (Kahn et al., 1964). With each role comes a set of rights, duties, expectations, norms, and behaviors specific to the context of the role. Practically speaking, almost everyone holds more than one role. In the industrialized world today, these roles are primarily divided up into domains, where some roles reside within the work domain and other roles reside outside the work domain.

What types of roles are included in work and nonwork domains? The construct of work has a primarily agreed-upon definition: it generally refers to a set of tasks that an individual performs in a proscribed position in a particular organization for a wage (Geurts \& Demerouti, 2003). Roles in the work domain may include supervisor, subordinate, coworker, line worker, or many more. Many individuals spend a large amount of their waking hours in the work domain, and for many, work roles such as job titles are part of how they define their sense of self (Geurts \& Demerouti, 2003). For example, an individual may gain a great deal of self worth based upon their work role as a doctor or a nurse.

Alternatively, roles may reside in the nonwork domain. There is much less consensus regarding how to operationalize the broad, and sometimes vague, area of life known as the nonwork domain. Many researchers choose to tackle this by examining a single portion of the nonwork domain, the family domain (Eby, Casper, Lockwood, 
Bordeaux, \& Brinley, 2005). This makes a great deal of sense, as many individuals have significant family responsibilities that take up the majority of their nonworking hours, and since family roles are another way that individuals often define their sense of self (Geurts \& Demerouti, 2003). For example, an individual might take great pride in being known as "Billy's father" or "Mr. Smith's wife”. Roles in the family domain may include parent, spouse, sibling, or child, among others. However, the nonwork domain is broader than simply family life. The nonwork domain encompasses activities and responsibilities not just in the family domain, but in other, nonwork, non-family areas of life, such as leisure time, household activities, and social obligations, among others (Geurts \& Demerouti, 2003). For example, an individual may be highly involved in activities with their faith or religion, and may strongly identify themselves as a Catholic or a Protestant. In order to gain a true picture of how individuals manage their multiple life roles, we must examine both the work domain and the broad domain of nonwork.

The boundaries between these two domains of work and nonwork are becoming blurred with the changing workforce. During the time period of industrialization, there was a major segregation between work roles and nonwork roles, where the two domains were spatially, temporally and mostly socially distinct (Wilensky, 1960). With the passage of time, the work and nonwork roles have become more highly interrelated, creating more issues regarding the boundaries between the two (Geurts \& Demerouti, 2003). For instance, an individual in today's world of work may conduct part of their work at home (telework), answer work calls after hours at home, have lunch with their child at the organization's on-site day-care, or take a personal call at their desk during 
working hours. Thus, the distinction between what is work and what is nonwork is more blurred than it once was. What remains clear is that individuals have multiple obligations and responsibilities in both their work domain and in their nonwork domain (Geurts \& Demerouti, 2003).

How do workers balance these multiple obligations and responsibilities for their work and nonwork domains? Several theories regarding this work-nonwork interface have been advanced, some with negative outcomes and other with positive outcomes. This dissertation will review three work-nonwork constructs: work-nonwork conflict, work-nonwork enhancement, and work-nonwork boundary fit.

Work-nonwork conflict. Early role theorists posited that the social expectations and demands for the work role were likely to be in conflict with those of the nonwork roles (Katz \& Kahn, 1966). That is, holding multiple roles should result in role conflict, a psychological tension aroused by conflicting role pressures (Katz \& Kahn, 1966). Based upon this structural-functionalist role theory, responsibilities and social scripts from multiple domains competed for limited amounts of time, physical energy, and psychological resources (Marshall, Chadwick, \& Marshall, 1991). The scarcity hypothesis (Goode, 1960) states that an individual has a finite amount of resources, and expending resources in one domain (work or nonwork) reduces the resources available in the other domain. This supports the "compensation" hypothesis of the relationship between work and nonwork, positing a negative association between work and nonwork (Staines, 1980). These structural-functionalist role theory perspectives proposed negative 
consequences of combining multiple roles, resulting in the definition of the concept of work-nonwork conflict (Marshall et al., 1991).

One of the first conceptualizations of this construct was elaborated by Greenhaus and Beutell (1985) as work-family conflict, a narrower version of the work-nonwork conflict construct examined here. These authors put forth that work-family conflict was defined as "a form of inter-role conflict in which the role pressures from the work and family domains are mutually incompatible in some respect. That is, participation in the work (family) role is made more difficult by virtue of participation in the family (work) role" (Greenhaus \& Beutell, 1985, p. 77). This definition also specified three forms of work-family conflict: time-based conflict, strain-based conflict, and behavior-based conflict. Time-based conflict occurs when the demands associated with one role take up a great deal of time, restricting the amount of time that can be dedicated to the other role. An example of this would be if an individual was staying late at work, which then made them miss the opportunity to eat dinner with their family. Strain-based conflict occurs when the demands of a particular role cause stress that interferes with the participation in the second role. An example of strain-based conflict would be if the demands of work caused an individual to be irritable and anxious when at home, thus hampering their ability to actively engage in enjoyable home activities. The last form of work-family conflict, behavior-based conflict, occurs when a particular behavior is encouraged in one role but is inappropriate in the other role. For example, authoritarian top-down decision making processes may be valued in the workplace, but create conflict if these decision- 
making processes are taken to the family domain where collaborative decision-making processes are expected (Greenhaus, Allen, \& Spector, 2006).

In the early 1990s, it was recognized that work-family conflict was reciprocal in nature, such that conflict can arise from work interfering with family, but also from family interfering with work (Gutek, Searle, \& Klepa, 1991). As such, Netemeyer, Boles, and McMurrian (1996) developed a scale that is widely used today to measure work interference with family (WIF) and family interference with work (FIW). Subsequent research supported the idea that the two directions of work-family conflict are related but distinct, with separate antecedents and outcomes, and only a modest correlation between the two subscales $(r=.30, r=-.55$; Frone, Russell, \& Cooper, 1992; Frone, Russell, \& Cooper, 1997; Netemeyer et al., 1996). Specifically, the antecedents of conflict stem primarily from the role that causes the interference, and the outcomes of conflict reside in the role that is the object of interference (Frone, 2003). For example, work demands predict WIF more strongly than family demands, but the major outcomes of WIF reside in the family domain rather than the work domain. Similarly, the majority of predictors of FIW stem from the family domain, but the outcomes lie primarily in the work domain (Greenhaus et al., 2006).

The concept of a negative relationship between work and nonwork domains has been called by many names, including work-home interference, negative spillover, workhome conflict, work-life conflict, and others. Some authors use the term "work/non-work conflict" as a synonym for "work-family conflict", citing the same definition by Greenhaus and Beutell in 1985 (e.g., Allan, Loudoun, \& Peetz, 2007; Rice, Frone, \& 
McFarlin, 1992; Wallace, 1999). These authors also cite identical antecedents and outcomes for work-nonwork conflict as those for work-family conflict. Other scholars argue that work-family conflict represents a narrower subset of the broader construct of work-nonwork conflict (e.g., Jones \& McKenna, 2002; Kreiner, 2006; Wallace, 1999). In this line of thinking, the terminology "work-family conflict" is socially limited to individuals with families, while the terms "work home conflict", "work-nonwork conflict", and "work-life conflict" are more broadly inclusive and less limiting in their definitions of the nonwork domain (Bulger et al., 2007; Jones \& McKenna, 2002; Kreiner, 2006).

In this study, I utilize the broader conceptualization of work-nonwork conflict. Considering the broader construct of work-nonwork conflict allows the examination of the relationship between work and all nonwork activities such as leisure, community involvement, or social activities that may include but are not restricted to the family domain. While family is an important part of life, individuals likely also hold other important roles and responsibilities that impact their experience of the intersection between work and nonwork (Fisher, Bulger, \& Smith, 2009). Utilizing this broad construct definition is especially necessary if we are to explore the experience of the work-nonwork interface for all workers. Changes in the composition of the workforce, such as increase in aging workers, make it less likely that every worker has a traditional family structure outside of work (Fisher et al., 2009). Additionally, Young (1999) suggested that the research focus on work and family, while admirable and worthy, may have created a work-family backlash based upon perceptions of unfairness or favoring 
those with children. She suggested that in order to counter this backlash, researchers and practitioners turn to more inclusive work-nonwork research, seeking solutions for all workers to gain work-nonwork balance. Consequently, the expansion of the definition allows examination of childless or unmarried workers who still may experience role conflict, but who are not traditionally captured in work-family research (Wallace, 1999). In recent years, several scholars have responded to this criticism by expanding their research to include more broad conceptualizations of the nonwork domains, such as work-life, work-home, or work-nonwork, rather than the narrower conceptualization of work-family (e.g., Fisher et al., 2009; Kreiner, 2006; Olson-Buchanan \& Boswell, 2006). This dissertation seeks to continue this trend by examining the work and nonwork domains and how to improve the balance for all types of workers.

Work-nonwork conflict as a predictor of health. How does work-nonwork conflict influence health? Several meta-analyses of the relationship between worknonwork conflict and health have found that effect sizes range from small but significant to medium (.13 to .35 ), depending on the direction of conflict and the indicator of health examined (Allen et al., 2000; Kossek \& Ozeki, 1999; Mesmer-Magnus \& Viswesvaran, 2005). For instance, in their meta-analysis of 67 articles, Allen and colleagues (2000) found that work interference with family was associated with several negative health outcomes, including general psychological health $(r=.29)$, somatic/physical symptoms $(r$ $=.29)$, depression $(r=.32)$, alcohol abuse $(r=.13)$, burnout $(r=.18)$, work-related stress $(r=.41)$, and family-related stress $(r=.31)$. Similarly, a newer meta-analysis of 98 articles found that work-to-nonwork conflict was significantly and positively related to 
health problems $(r=.28)$, psychological strain $(r=.35)$, somatic/physical symptoms $(r$ $=.29)$, depression $(r=.23)$, substance use/abuse $(r=.08)$, stress $(r=.54)$, and anxiety $(r$ $=.14 ;$ Amstad, Meier, Fasel, Elfering, \& Semmer, 2011). The same meta found that nonwork-to-work conflict was significantly and positively related to these same health indicators, including health problems $(r=.24)$, psychological strain $(r=.21)$, somatic/physical symptoms $(r=.14)$, depression $(r=.22)$, substance use/abuse $(r=.10)$, stress $(r=.39)$ and anxiety $(r=.19$; Amstad et al., 2011).

More specifically, previous research has shown that work-nonwork conflict is positively related to physical symptoms and strain, as well as negatively related to overall physical health (Allen et al., 2000). Work-nonwork conflict has been shown to be positively related to several specific health complaints and diseases as well, including hypertension, diastolic blood pressure, obesity, and cholesterol levels (Frone et al., 1997; Thomas \& Ganster, 1995; Van Steenbergen \& Ellemers, 2009). Additionally, worknonwork conflict has been associated with unhealthy behaviors, such as substance dependence, tobacco use, extensive medication use, alcohol use, low levels of physical exercise, and poor food choices (Frone, 2003; Greenhaus et al., 2006).

Mental health also suffers as a result of work-nonwork conflict, including high levels of depression, mood disorders, and anxiety (Frone, 2000; Frone et al., 1997; Grzywacz \& Bass, 2003). Work-nonwork conflict appears to increase the presence of negative emotions such as life stress and psychological strain (Greenhaus et al., 2006). There is strong evidence that high levels of work-nonwork conflict are linked to lowered levels of life satisfaction (Carlson \& Kacmar, 2000; Greenhaus, Collins, \& Shaw, 2003; 
Netemeyer et al., 1996; Parasuraman, Greenhaus, \& Granrose, 1992; Perrewe, Hochwarter, \& Kiewitz, 1999). Combining all of this research makes it clear: there can be dire health results if employees suffer from elevated levels of work-nonwork conflict.

Predictors of work-nonwork conflict. The research listed above makes it clear that work-nonwork conflict can be extremely harmful to health of workers, and should be avoided at all costs. So how can we lower or eliminate work-nonwork conflict? Previous research has identified several predictors of work-nonwork conflict that are controllable and changeable, and can be used to reduce work nonwork conflict. These predictors can stem from the work domain as well as other nonwork domain aspects.

Work domain predictors of work-nonwork conflict. Certain work domain characteristics have been shown to predict work-nonwork conflict. As mentioned previously, work domain characteristics can influence both directions of work-nonwork conflict, but generally have the strongest impact on conflict from the workplace to other domains (Ford, Heinen, \& Langkamer, 2007). The number of hours spent on the job, and the time at which those hours are spent on the job can also influence work-nonwork conflict. For example, long hours have been shown to have a positive relationship with instances of work-nonwork conflict (Carlson \& Perrewe, 1999; Ford et al., 2007; Greenhaus, Bedeian, \& Mossholder, 1987), as does working nonstandard shifts (Fox \& Dwyer, 1999). What individuals do during those hours can also impact work-nonwork conflict. For example, individuals with high job involvement are more likely to suffer from work-nonwork conflict (Carlson \& Perrewe, 1999; Ford et al., 2007). Individuals with higher levels of work demands, more work stress, and greater time commitment are 
also likely to suffer from work-nonwork conflict (e.g., Carlson, 1999; Ford et al., 2007; Grzywacz \& Marks, 2000). Not surprisingly, this is especially relevant to those that are self-employed (Parasuraman \& Simmers, 2001).

Not all work characteristics are bad for work-nonwork conflict, however. High levels of perceived organizational support can greatly reduce work-nonwork conflict (Carlson \& Perrewe, 1999; Greenhaus et al., 1987). A workplace that offers familyfriendly benefits can allow individuals to be sure their loved ones are cared for, thereby lowering work-nonwork conflict (Thompson, Beauvais, \& Lyness, 1999). Similarly, employees who have a great deal of control or autonomy at work are likely to be able to exert influence over their work environment to make it more rewarding, less threatening, or less likely to interfere with nonwork activities, thereby reducing work-nonwork conflict (Clark, 2002; Perrewe, Treadway, \& Hall, 2003). This control may manifest itself in terms of ability to work flexible hours, which consequently reduces worknonwork conflict (Thomas \& Ganster, 1995). Leaders can be especially instrumental in lowering work-nonwork conflict. Supervisors or mentors that are supportive, model positive behaviors, and are perceived to have similar work-family values can lower worknonwork conflict in their subordinates (Lapierre \& Allen, 2006; Nielson, \& Carlson, Lankau, 2001; Thomas \& Ganster, 1995). Supervisors that behave in ways that are especially supportive of families, such as giving modeling positive work-nonwork behaviors and engaging in creative work-family management, can reduce work-nonwork conflict over and above general supportiveness (Hammer, Kossek, Yragui, Bodner, \& Hanson, 2009). In sum, there are many aspects of the work environment or 
characteristics of the work that can be altered to reduce work-nonwork conflict and improve health.

Nonwork domain predictors of work-nonwork conflict. Aspects of the nonwork domain can also influence work-nonwork conflict. As mentioned previously, while these nonwork domain characteristics can impact both directions of work-nonwork conflict, they are most likely to strongly influence conflict from nonwork domains to work domains (Ford et al., 2007). Certain family characteristics, such as the number of children at home or the presence of childcare problems, can increase work-nonwork conflict (Carlson, 1999; Grzywacz \& Marks, 2000; Fox \& Dwyer, 1999). Similar to the research in the work domain, high levels of involvement with family or other nonwork domains can increase work-nonwork conflict, especially in the nonwork-to-work direction (Carlson \& Perrewe, 1999; Ford et al., 2007; Parasurman \& Simmers, 2001). High levels of stress in nonwork domains, such as tension or stress within a family, can also increase work-nonwork conflict (Carlson \& Perrewe, 1999; Ford et al., 2007). Similarly, individuals who are highly involved in their nonwork domains are likely to suffer higher levels of work-nonwork conflict, especially in the nonwork-to-work direction (Carlson \& Perrewe, 1999).

Similar to research conducted on work predictors of work-nonwork conflict, not all aspects of the nonwork domain are detrimental to work-nonwork conflict, either. The presence of support from spouses or other family members can reduce nonwork-to-work conflict (Grzywacz \& Marks, 2000; Matthews, Bulger, \& Barnes-Farrell, 2010). 
Additionally, some nonwork activities, such as volunteering, can lower work-nonwork conflict (Hecht \& Boies, 2009).

Certain individual differences can also impact work-nonwork conflict. Individuals with less negative affect also report less work-nonwork conflict (Carlson, 1999). Similarly, individuals with Type A personalities also have lower levels of worknonwork conflict (Carlson, 1999). Neurotic personalities are more likely to have both directions of work-nonwork conflict, while extroverts are less likely to experience conflict from the work domain to other domains (Grzywacz \& Marks, 2000).

In sum, previous empirical research indicates that work-nonwork conflict is a viable predictor of health, and that work-nonwork conflict can be altered or changed by a variety of factors residing in multiple domains. Thus,

Hypothesis 1a: Work-to-nonwork conflict will be negatively related to both physical and mental health over time.

Hypothesis 1b: Nonwork-to-work conflict will be negatively related to both physical and mental health over time.

Work-nonwork enhancement. The literature on work-nonwork conflict makes the intersection between work and nonwork domains appear to be a risky combination, fraught with negative outcomes. However, this does not represent the entire picture of the interface between work and nonwork domains. To gain a balanced perspective regarding this interface, we must also examine the positive side of the work-nonwork interface. 
The positive spillover or role enhancement perspective posits that work and nonwork domains may be mutually beneficial, due to the spillover of positive experiences from one role to another (Edwards \& Rothbard, 2000; Greenhaus \& Powell, 2006). This conceptualization of the work-nonwork interface is based on the enhancement hypothesis, which states that participation in multiple roles can provide a greater number of opportunities and resources to the individual that can then be used to enhance functioning in other domains (Marks, 1977). Marks (1977) states that "some roles may be performed without any net energy loss at all; they may even create energy for use in that role or in other role performances (Marks, 1977, p. 926). This indicates that workers' activities and actions in nonwork domains may energize them for their work, as well as visa versa. These benefits may stem from a gain in resources, positive affect, or psychological capital gained from one role that can transfer to the other role (Sui et al., 2010). Previous research seems to support this hypothesis, showing that holding more roles is associated with higher self-esteem, greater job satisfaction, and lowered psychological distress (Pietromonaco, Manis, Frohardt-Lan, 1986; Thoits, 1983).

Operational terminology for this positive side of the work-nonwork interface varies, as it has been known by terms including facilitation (Grzywacz, Almeida, \& McDonald, 2002), enrichment (Greenhaus \& Powell, 2006; Carlson, Kacmar, Wayne, \& Grzywacz, 2006), enhancement (Gordon, Whelan-Berry, \& Hamilton, 2007; Voydanoff, 2002), and positive spillover (Crouter, 1984; Edwards \& Rothbard, 2000). For many researchers, these terms are synonyms for the concept that multiple domains can be mutually beneficial. For other researchers, there are slight definitional distinctions 
between the terms. For instance, some researchers define facilitation as the extent to which involvement in one domain provides gains that contribute to functioning in another domain (Wayne, Grzywacz, Carlson, \& Kacmar, 2007), while enrichment is the degree to which experiences in one role improve the quality of life in the other role (Greenhaus \& Powell, 2006). For the purposes of this dissertation, I will refer to the overarching concept of mutually beneficial domains as "enhancement".

In the past, the most popular way to examine this construct of enhancement or enrichment is through work and family domains, rather than more broad domains of work and nonwork (e.g., Hanson, Hammer, \& Colton, 2006). Similar to the literature regarding conflict, work-family enhancement can be conceptualized as a narrower subsection of work-nonwork enhancement. This narrow construct definition implies a sample restriction to only those who have a traditionally defined family, i.e., a spouse and children. In order to be more inclusive in my sample and my study, I chose to study the broader conceptualization of work-nonwork enhancement. Logically, expanding from the family domain to broader nonwork domains should be in the spirit of enhancement, as this definitional expansion allows examination of a greater number of roles, which should in theory result in greater positive outcomes. For instance, Kirchmeyer (1995) found that each nonwork domain had its unique set of roles that enhanced work differently than other domains. Her participants believed that parenting provided unique rewards and a strong buffer against work problems, while community work strongly increased an individual's value and work and provided ideas for work, and recreation most strongly impacted energy and the ability to forget work problems (Kirchmeyer, 1995). This 
research shows the value in considering the positive spillover from all non-work domains, rather than just the family role. Thus, this study examines the broader concept of work-nonwork enhancement and how it can influence health.

How does work-nonwork enhancement occur? Edwards and Rothbard (2000) posit that there are four types of enhancement or spillover: moods, values, skills, and behaviors. Mood enhancement occurs when the mood generated by one domain positively impacts the mood in the other domain, increasing the probability of additional positive interactions, such as happiness or optimism. This is sometimes known as affective work-nonwork enhancement (Greenhaus \& Powell, 2006; Hanson et al., 2006). Value enhancement can occur when values originating in one domain positively influence values in another domain, such as curiosity. Skills enhancement occurs when an individual learns valuable skills in one domain that are transferable and applicable to another domain, such as money management. Behavioral enhancement occurs when an individual learns an adaptive behavior in one role that can be transferred to another role with positive outcomes, such as collaborative problem-solving (Edwards \& Rothbard, 2000). These latter three types of enhancement can be described as instrumental worknonwork enhancement (Greenhaus \& Powell, 2006; Hanson et al., 2006).

Recent developments have shown that, similar to work-nonwork conflict, worknonwork enhancement is bidirectional in nature. That is, the enhancement can come from the work domain to other domains, or from outside domains into the work domain (Greenhaus \& Powell, 2006). Again paralleling the work-nonwork conflict structure, recent evidence shows that each direction of work-nonwork enhancement has distinct 
antecedents and outcomes (Grzywacz \& Marks, 2000). Additionally, recent research has indicated that work-nonwork conflict and work-nonwork enhancement are not mutually exclusive concepts, and that each process may operate simultaneously, depending on individual differences, work domain characteristics, and nonwork domain characteristics (Geurts \& Demerouti, 2003). In other words, when work and nonwork domains interact in a way that produces conflict, it does not also rule out interactions that may produce enhancement. However, it is easily apparent that the ideal state occurs when little to no conflict arises between the two domains and much enhancement occurs between the two domains.

Work-nonwork enhancement as a predictor of health. How does work-nonwork enhancement impact health? In theory, work-nonwork enhancement creates positive emotions, which in turn promotes flexibility, creativity, and proactivity, which contribute to personal thriving and overall health (Van Steenbergen \& Ellemers, 2009). Worknonwork enhancement also creates affective or instrumental resources across domains that can lead to more energy, which allows individuals to engage in healthy behaviors (Greenhaus \& Powell, 2006). Previous empirical research has supported the idea that work-nonwork enhancement is linked to improved health. For example, a recent metaanalysis suggested that both directions of work-nonwork enhancement have a positive relationship with overall physical and mental health (McNall, Nicklin, \& Masuda, 2010). Work-nonwork enhancement has been linked to the presence of positive health aspects, such as positive psychological well-being (Carlson et al., 2006), positive mental health (Hanson et al., 2006), and good sleep quality (Williams, Franche, Ibrahim, Mustard, \& 
Layton, 2006). Work-nonwork enhancement has also been linked to the absence or reduction of negative health outcomes, including fewer depressive symptoms (Grzywacz \& Bass, 2003; Hammer, Cullen, Neal, Sinclair, \& Shafiro, 2005), lower levels of psychological distress (Haar \& Bardoel, 2008), fewer chronic health problems (Grzywacz, 2000), lowered cholesterol levels, and lowered body-mass index (BMI) levels (Van Steenbergen \& Ellemers, 2009). One study by Grzywacz and Bass (2000) found that a one-unit increase in work-nonwork enhancement was associated with a $15 \%$ decrease in depression and a $38 \%$ decrease in problem drinking. Overall, this previous research supports the idea that work-nonwork enhancement is a positive predictor of health, although this relationship has not been examined as extensively as the worknonwork conflict and well-being relationship has.

Predictors of work-nonwork enhancement. Thus, previous empirical research seems to indicate that work-nonwork enhancement is a desirable predictor of health. How can organizations increase the work-nonwork enhancement of their employees? Previous empirical research indicates that certain characteristics and attributes of both the work and nonwork domains can influence levels of work-nonwork enhancement.

Work domain predictors of work-nonwork enhancement. Certain work characteristics can influence an individual's experience of work-nonwork enhancement. For instance, high pressure at work is associated with decreased work-nonwork enhancement in both directions (Grzywacz \& Marks, 2000). However, there are many things an organization can do to improve their workers' levels of work-nonwork enhancement. For example, a workplace that offers family-friendly organizational 
policies is likely to result in workers with increased levels of work to nonwork enhancement (Sui et al., 2010). Individuals who have a great deal of job autonomy or decision latitude are also likely to have work-nonwork enhancement in both directions (Grzywacz \& Marks, 2000; Sui et al., 2010). Supportive supervisors can increase the likelihood that their subordinates will experience work-nonwork enhancement (Sui et al., 2010; Thompson \& Prottas, 2006). This is especially true when supervisors exhibit specific family supportive behaviors, rather than just general support (Hammer et al., 2009). Support from coworkers can also lead to high levels of work-nonwork enhancement (Thompson \& Prottas, 2006). Overall, these sources of support can lead to a workplace that has an informal culture of supporting individuals with their nonwork lives, especially their family domain. This family-supportive culture can increase instances of work-nonwork enhancement (Wayne, Randel, \& Stevens, 2006).

Nonwork domain predictors of work-nonwork enhancement. Characteristics of various nonwork domains can also influence work-nonwork enhancement. The majority of this research pertains to the family domain, and within that, the importance of the role of a spouse or partner.

Marital status is a predictor of enhancement, such that married individuals have higher levels of enhancement than unmarried individuals (Grzywacz \& Marks, 2000). Individuals who are satisfied with their relationships appear to also have elevated levels of enhancement, at least for men (Stevens et al., 2007).

How might the presence of a spouse improve an individual's levels of worknonwork enhancement? One mechanism is via support. Previous research has shown 
that spousal support, especially emotional support, is positively related to an individual's levels of work-nonwork enhancement (Aryee, Srivivas, \& Tan, 2005; Kinnunen, Feldt, Geurts, \& Pulkkinen, 2006; Sui et al., 2010; Wayne, Randel, \& Stevens, 2006).

Another reason for the beneficial effects of a spouse may be due to the spouse's own experienced enhancement. One recent study examined the positive spillovercrossover model in dual-earner couples (Demerouti, 2012). The results showed that when one partner, Partner A, experiences positive resources at work, it leads to high levels of work-to-nonwork enhancement, which creates increased levels of energy in Partner A. Partner A then brings this increased energy into the home domain, which allows him or her to contribute more to the demands and responsibilities of the home domain. Thus, Partner B's perception of his or her home resources are increased, resulting in his or her own experience of nonwork-to-work enhancement and a subsequent increase in energy that can be utilized in the workplace (Demerouti, 2012). Thus, an individual's experience of enhancement appears to positively influence their partner's experience of enhancement as well.

Other aspects of the family domain can also positively influence an individual's experience of work-nonwork conflict. Support from other family members, not just spouses, can increase enhancement levels (Aryee, Srivivas, \& Tan, 2005; Kinnunen, Feldt, Geurts, \& Pulkkinen, 2006; Sui et al., 2010; Wayne, Randel, \& Stevens, 2006). Family cohesion is also positively related to work-nonwork enhancement (Stevens, Minnotte, Mannon, \& Kiger, 2007). 
There is limited research on non-work/non-family predictors of enhancement. However, one study found that involvement in certain nonwork activities, specifically team sports and volunteering, can increase nonwork-to-work positive spillover, indicating that work-nonwork enhancement can be influenced by factors outside the family domain (Hecht \& Boies, 2009).

In sum, theory and previous empirical research support the idea that worknonwork enhancement can influence health, and that work-nonwork enhancement itself can be changed and altered. Thus,

Hypothesis 2a: Work-to-nonwork behavior-based enhancement will be positively related to both physical and mental health over time.

Hypothesis 2b: Work-to-nonwork value-based enhancement will be positively related to both physical and mental health over time.

Hypothesis 2c: Work-to-nonwork affective enhancement will be positively related to both physical and mental health over time.

Hypothesis 2d: Nonwork-to-work behavior-based enhancement will be positively related to both physical and mental health over time.

Hypothesis 2e: Nonwork-to-work value-based enhancement will be positively related to both physical and mental health over time.

Hypothesis 2f: Nonwork-to-work affective enhancement will be positively related to both physical and mental health over time.

Work-nonwork boundary fit. The two theories and corresponding constructs presented above make it clear that work and nonwork domains do, in fact, interact, and 
that the results of this interaction can be detrimental (conflict) or beneficial (enhancement). Implicit in both theories is the idea that work and nonwork domains are separate, individualized domains, and that boundaries are present to keep the domains separate. It is this concept of boundaries between domains, rather than consequences of combining domains, that we examine now. Two theories address the presence of these boundaries: boundary theory (Nippert-Eng, 1996) and work-family border theory (Clark, 2000).

Boundary theory was originally developed as a cognitive sociological perspective used to understand how individuals classify any set of entities into bounded categories (Zerubavel, 1991). This theory was adapted for the work-family field by Nippert-Eng (1996), and further refined to focus on developing testable models of role transitions (Ashforth, Kreiner, \& Fugate, 2000) and the interactions between boundary preferences and available employer policies (Kreiner, 2002). Boundary theory examines how boundaries are established and maintained when an individual is active in multiple life domains (Ashforth et al., 2000). The principle behind boundary theory is that individuals deliberately create boundaries around their work and personal life, and that the construction of these boundaries depends on a multitude of factors, including occupation type and individual preference (Nippert-Eng, 1996). Boundary theory states that cognitive, physical, and/or behavioral boundaries exist between an individual's work and nonwork domains (Ashforth et al., 2000; Clark, 2000).

Work-family border theory was developed to address how boundaries divide the times, places, and people that are associated with each role (Clark, 2000). Work-family 
border theory proposes that work-family balance is an ideal state where an individual experiences "satisfaction and good functioning at work and at home, with a minimum of role conflict" (Clark, 2000, p. 751). This balance depends on characteristics of the individual and the environment, such as the similarity of the work and home domains and the strength of the boundaries between these domains.

Work-family border theory and boundary theory have many similarities. Both theories state that strategies for managing multiple domains can vary on a continuum from highly segmented to highly integrated (Ashforth et al., 2000; Kreiner, 2006; Nippert-Eng, 1996). Highly segmented domains occur when work and nonwork domains rarely overlap; when an individual is physically in one domain, they are not mentally or behaviorally active in another. In this scenario, boundaries between the two domains are rarely crossed. In contrast, highly integrated domains allow an individual to address issues or activities in one domain, such as family, while physically present in another domain, such as work. In this scenario, boundaries between the two domains are crossed frequently and easily. It has been proposed that highly segmented domains preserve role clarity between domains, but may sacrifice ease of transitions between roles (Ashforth et al., 2000). Conversely, highly integrated domains may facilitate ease of transitions between roles, but increase role blurring or confusion (Ashforth et al., 2000).

Both work-family border theory and boundary theory posit that the strength of a particular boundary can be characterized by permeability and flexibility. A permeable boundary exists when elements from one domain are readily found in the other domain (Ashforth et al., 2000; Clark, 2000). This may include actual interruptions or intrusions 
from one domain into the other that may not be under the control of the employee. Permeability occurs when an individual is physically located in one domain but behaviorally engaged in a different domain (Bulger, Matthews, \& Hoffman, 2007). The other dimension of boundary strength, flexibility, is the hypothetical or perceived capacity to strengthen or weaken a particular boundary based on the needs of another domain (Matthews, Barnes-Farrell, \& Bulger, 2010). This includes both an individuals' willingness to flex boundaries as well as the individual's ability to do so.

According to recent research utilizing work-family border theory and boundary theory, there is no universally adaptive border management strategy. In other words, neither integration nor segmentation is "best" for individual outcomes. Rather, the optimum solution is influenced by a variety of factors. Chief among these is the individual preference for border characteristics. Individuals vary in the degree to which they prefer to segment or integrate their work and nonwork domains (Rothbard, Phillips, \& Dumas, 2005). These preferences can be formed by employee characteristics such as time-management skills, social influence at home and at work, and the personal importance they attach to each role (Desrochers, Hilton, Larwood, 2005). Individuals choose their segmentation/integration strategy in order to best minimize the difficulty of balancing both roles (Ashforth et al., 2000). In the literature, this concept is referred to as segmentation preference.

Additionally, differences exist in the degree to which a particular workplace or organization supplies or provides an environment that allows or permits employees to segment their work and nonwork affairs (Chen et al., 2009). This can be embodied by 
the organizational climate and culture surrounding work-nonwork boundaries, including both formal work-family policies, such as the availability of onsite childcare, and informal expectations, such as the unstated expectation that individuals will be available for phone calls at any time of the day or night (Carr, Schmidt, Ford, \& DeShon, 2003). In the literature, the individual's perception of this organizational climate for work-nonwork boundary management is referred to as segmentation supplies, or the degree to which the individual perceives that the organization promotes segmentation or integration of domains.

The congruence between segmentation preferences and segmentation supplies has been examined using a person-environment (P-E) fit theoretical lens (Chen et al., 2009; Kreiner, Hollensbe, \& Sheep, 2006). Strong P-E fit occurs when there is compatibility between an individual and his or her environmental characteristics (Kristof-Brown \& Guay, 2011). Fit can exist on any range of personal attributes (such as needs, traits, goals, values, or, in this case, boundary preferences) and environmental attributes (such as job demands, working conditions, organizational culture, all of which can contribute to organizational supplies; Kristof-Brown \& Guay, 2011). P-E fit theory posits that stress occurs when there is a perceived mismatch between the environment and an individual's preferences, goals, or values (Edwards \& Rothbard, 1999).

There are two research streams concerning what constitutes "good fit". Unfortunately, these two research streams are characterized by a lack of integration (Kristof, 1996). One research stream focuses on supplementary fit, which emphasizes the similarity between the person and his or her environment. In this paradigm, values are 
beliefs that pertain to desirable end states, guide behavior, and vary in terms of relative importance (Schwartz, 1992). Thus, individual values guide behavior, and organizational value systems create norms that specify how resources should be allocated and how organizational members should behave (Cable \& Edwards, 2004). Value congruence occurs when there is a similarity between an individual's values and the value system of the organization (Kristof, 1996). In short, supplementary fit assumes that similarity begets compatibility. In theory, value congruence creates positive outcomes because individuals are attracted to those who are similar to them (e.g., Byrne, 1969; Tsui \& O’Reilly, 1989). Thus, employees are likely to be comfortable working for organizations where their values are mirrored in other employees (Cable \& Edwards, 2004). This similarity should improve communication and other interpersonal relationships by reducing uncertainty (Kalliath, Bluedorn, \& Strube, 1999). Instances where values are not reflected by the organization create cognitive dissonance and poor reactions (O'Reilly, Chatman, \& Caldwell, 1991). An example of research conducted under this paradigm is research examining value congruence between employees and organizations (Kristof, 1996).

The second stream of research focuses on complementary fit, where good fit occurs when each aspect of the dyad (person and environment) meets the needs of the other, while not necessarily being similar (Cable \& Edwards, 2004; Muchinsky \& Monahan, 1987). In this paradigm, employees have certain psychological needs acquired through learning and socialization, which are then compared to the environmental supplies, or extrinsic and intrinsic resources and rewards, provided by the organization to 
meet those needs (Cable \& Edwards, 2004). An individual's perceptions of needs and supplies are central to this theory, as a person can react to misfit between needs and supplies only if he or she is aware that misfit exists (e.g., Edwards, Caplan, \& Harrison, 1998). In short, the underlying process is an individual's cognitive comparison of the desired amount of a certain resource relative to the amount of that resource that is supplied by the organization (French, Caplan, \& Harrison, 1982). Individuals become dissatisfied when the supplies provided by the employer do not meet the individual's desired level. Satisfaction occurs as supplies increase toward desires. Research has shown that instances of excess supplies can result in variety of reactions, depending on the specific needs and desires examined (Cable \& Edwards, 2004). Much of the research conducted in the complementary perspective has been in regards to psychological need fulfillment (Edwards, 1991). This research examines how individual's attitudes are impacted by the fit between their desires and the supplies in the work environment that are available to meet those desires.

Within complementary fit, there is a further distinction between needs-supplies fit and demands-abilities fit (Caplan, 1987; Edwards, 1991; Kristof, 1996). The needssupplies perspective states that P-E fit occurs when the organization fulfills the individual's needs or wants. In this paradigm, organizations supply financial, physical, and psychological resources to meet employee needs (Kristof, 1996). Conversely, the demands-abilities perspective states that fit occurs when the individual's abilities satisfy organizational demands. These demands may include time, effort, knowledge, skills, and abilities (Kristof, 1996). 
The current study seeks to examine the concept of fit in terms of complementary fit examined through the needs-supplies perspective; that is, individual's perceptions of how the organization meets their work-nonwork boundary needs and desires. This is in line with previous research on the topic and previous use of the proposed measures (e.g., Chen et al., 2009; Kreiner, 2006).

Regardless of the particular operationalization of what constitutes good fit, it is clear that good fit produces positive outcomes. When there is good fit between a person and his or her environment, favorable outcomes occur for both the organization and the individual (Cable \& Edwards, 2004). Previous research has linked P-E fit to desirable outcomes such as job satisfaction, organizational commitment, satisfaction with coworkers, feelings of cohesion within a team, satisfaction with supervisors, lower level of stress, higher contextual performance, lower levels of turnover intentions (KristofBrown \& Guay, 2011).

When applied to the concept of work-nonwork boundaries, an individual's segmentation preferences is the "person", while the organization's segmentation supplies constitutes the "environment" (Chen et al., 2009). Thus, a misfit between an individual's segmentation preferences and the segmentation supplies provided by the organization should theoretically result in high stress levels and poor outcomes for both individuals and organizations, while positive outcomes should theoretically arise from instances of good fit (Kristof-Brown \& Guay, 2011). Recent research supports the idea that work-life fit is a strong predictor of important workplace outcomes. For example, the recent Workplace Retention Survey found that work-life fit was a very important predictor of 
turnover (APA, 2012). In fact, work-life fit topped the list of reasons for remaining with one's current employer, tying with "I enjoy the work I do" at $67 \%$ of respondents, and beating out other popular reasons such as benefits (60\%) and pay (59\%; APA, 2012).

Work-nonwork boundary fit as a predictor of health. Previous research using the theoretical lens of P-E fit to examine boundary congruence has supported this notion that good fit leads to positive outcomes. Specifically, some research has shown that good fit is beneficial for health. Edwards and Rothbard (1999) examined autonomy, relationships, security, and segmentation "values" (i.e., individual preferences) compared to "supplies" for those same constructs (i.e., available levels of those variables in the workplace, such as human resource policies on flextime, flexplace, and other formal and informal policies). Their results show that well-being (as measured by satisfaction, depression, anxiety, irritation, and somatic symptoms) increased as supplies approached the values and then decreased as supplies exceeded the values. This indicates that the highest well-being is produced when individual preferences for work-nonwork boundary conditions are met, and that too little or too much segmentation beyond that preferred level is detrimental to well-being. The authors found that this relationship primarily impacted affective indices of well-being, such as satisfaction, depression, and irritation, rather than the other well-being outcomes of anxiety and somatic symptoms.

While the Edwards and Rothbard (1999) study provided encouraging evidence that work-nonwork boundary fit is a predictor of health and well-being, the study was cross-sectional, making causal inferences difficult. Additionally, the sample used was all from a single organization (a large university). This sample restriction may limit the 
generalizability of the results to other organizations or occupations. The current study addresses both of these limitations by examining the phenomena at two time points using a sample drawn from a wide variety of organizations and industries. Additionally, this study adds to the knowledge gained from the Edwards and Rothbard (1999) study by examining outcome measures of overall mental and physical health, rather than specific mental illnesses. Thus,

Hypothesis 3a: Good fit between individual work-nonwork boundary management preferences and organizational work-nonwork boundary management supplies will be positively related to mental health over time.

Hypothesis 3b: Good fit between individual work-nonwork boundary management preferences and organizational work-nonwork boundary management supplies will be positively related to physical health over time.

\section{Indirect Effects}

The theory and previous empirical research presented in the preceding sections have shown that three aspects of the work-nonwork interface (work-nonwork conflict, work-nonwork enhancement, and work-nonwork boundary fit) are viable predictors of health. However, no argument has yet been made for how these three constructs relate to each other, and to the ultimate outcome of interest, health. Logically, how do worknonwork conflict, work-nonwork enhancement, and work-nonwork boundary fit relate to one another?

In order to answer this question, we must first consider what each of these constructs includes. Work-nonwork boundary fit, as previously discussed, is made up of 
the individual's preferences for work-nonwork boundary management and the organization's supplies for work-nonwork boundary management. Organizational boundary supplies are reflective of the culture or the climate of the workplace in regards to employee boundary management. Thinking of work-nonwork boundary fit in this way, the construct implies a snapshot of how the employee perceives the workplace in comparison to how they would ideally want their workplace to function. In short, it describes aspects of the work environment.

Work-nonwork conflict and work-nonwork enhancement, alternatively, are constructs that describe what occurs when work and nonwork domains intersect. Thus, it makes logical sense that characteristics of the workplace must precede outcomes of the workplace interacting with other roles and responsibilities. In this manner, it seems that work-nonwork boundary fit is conceptually prior to work-nonwork conflict and worknonwork enhancement. This ordering leads me to propose that the influence of worknonwork boundary fit on health and well-being occurs not only directly, but also indirectly through the mechanisms of work-nonwork conflict and work-nonwork enhancement.

By definition, indirect effects such as those proposed above posit that $\mathrm{X}$ is related to $\mathrm{Y}$ via a mechanism $\mathrm{M}$, such that $\mathrm{X} \rightarrow \mathrm{M} \rightarrow \mathrm{Y}$ (Mathieu, DeShon, \& Bergh, 2008). In order for a variable to be proposed as a likely mechanism of a relationship, there should be evidence of three things: 1) X predicts $Y, 2) X$ predicts $M$, and 3) M predicts $Y$. Thus, the previous research presented in this dissertation proposal has shown that worknonwork boundary fit, work-nonwork conflict and work-nonwork enhancement do 
indeed predict health and well-being, fulfilling both the "X predicts $\mathrm{Y}$ " and the " $\mathrm{M}$ predicts $\mathrm{Y}^{\prime}$ requirement. The remaining question is whether $\mathrm{X}$, in this case, worknonwork boundary fit, predicts both work-nonwork conflict and work-nonwork enhancement, the mechanism.

Work-nonwork conflict as a mechanism. Let us first examine the construct of work-nonwork conflict as a potential mechanism between work-nonwork boundary fit and health. Logically, it makes sense that the fit between an individual's work-nonwork boundary preferences and the organization's practices and policies surrounding worknonwork boundary management would have an impact on work-nonwork conflict. If an individual felt that the organizational boundary management supplies matched well with their boundary preferences, this implies that the individual would be free to manage their work and nonwork lives as they wished, resulting in a state without conflict between the two.

Previous research seems to support this theoretical relationship, such that good fit does indeed relate negatively to work-nonwork conflict. For example, Chen and colleagues (2009) found that individuals experienced less work-family conflict when there was a good fit between their preferences for work-family segmentation and the supplies for segmentation provided by the workplace, supporting logic and theory. Similarly, Kreiner (2006) examined the impact of the perceived fit between an individual's work-home segmentation preference and the perceived supplies of segmentation provided by the work-environment on work-home conflict, stress, and job satisfaction using a P-E fit theoretical base and a longitudinal survey design. Results 
showed that as segmentation supplies increased toward preferences, work-home conflict decreased, indicating that positive fit between segmentation preferences and organizational segmentation supplies reduced instances of work-nonwork conflict.

In sum, it appears that the fit between individual boundary preferences and organizational boundary management supplies do indeed impact work-nonwork conflict. As predicted by P-E fit theory, situations of good fit tend to result in lower levels of conflict. Additionally, as reviewed previously in this dissertation proposal, worknonwork conflict has been shown to predict a variety of health outcomes. Thus, it appears that work-nonwork conflict may indeed be a viable mechanism of the worknonwork boundary fit-health and health relationship. Following that,

Hypothesis 4a: There will be a significant positive indirect effect of worknonwork boundary fit on both physical and mental health through work-tononwork conflict.

Hypothesis 4b: There will be a significant positive indirect effect of worknonwork boundary fit on both physical and mental health through nonwork-towork conflict.

Work-nonwork enhancement as a mechanism. Let us now move to examining the construct of work-nonwork enhancement as a potential mechanism of the worknonwork boundary fit-health and health relationship. Assuming that a good fit between individual preferences and organizational supplies allows workers to manage their worknonwork boundaries in the way they prefer, it is logical to assume that this ideal 
boundary management situation would create positive moods, values, skills and behaviors that could spill over between domains. Does the research support this idea?

Previous research indicates that the level of congruence between segmentation preferences and supplies relates positively to individual experiences of work-nonwork enhancement. Chen and colleagues (2009) found that employees who have a good fit between their work-nonwork boundary preferences and their workplace supplies have higher levels of instrumental work-family positive spillover, indicating that when individuals' preferences for boundary control is being met, it may generate additional resources that may be applied toward improving their family lives. However, they did find that, counter to their hypotheses, there was a significant negative effect of boundary fit on affective work-family spillover.

In sum, it appears that work-nonwork boundary fit does indeed positively predict work-nonwork enhancement, which in turn positively predicts health and well-being. Thus,

Hypothesis 5a: There will be a significant positive indirect effect of worknonwork boundary fit on both physical and mental health through work-tononwork behavior-based enhancement.

Hypothesis 5b: There will be a significant positive indirect effect of worknonwork boundary fit on both physical and mental health through work-tononwork value-based enhancement. 
Hypothesis 5c: There will be a significant positive indirect effect of worknonwork boundary fit on both physical and mental health through work-tononwork affective enhancement.

Hypothesis 5d: There will be a significant positive indirect effect of worknonwork boundary fit on both physical and mental health through nonwork-towork behavior-based enhancement.

Hypothesis 5e: There will be a significant positive indirect effect of work-nonwork boundary fit on both physical and mental health through nonwork-to-work valuebased enhancement.

Hypothesis 5f: There will be a significant positive indirect effect of work-nonwork boundary fit on both physical and mental health through nonwork-to-work affective enhancement.

\section{Research Question}

As described earlier, previous research has indicated that work-nonwork boundary fit is an important predictor of several outcomes, including work-nonwork conflict, worknonwork enhancement, and overall health. Interestingly, many of these studies that examined the concept of work-nonwork boundary fit discovered unexpected findings regarding the importance of boundary segmentation level as a predictor. Specifically, boundaries that are more highly segmented (rather than more highly integrated) seem to be beneficial for these same outcomes.

For example, in their study of work-nonwork boundary fit, Edwards and Rothbard (1999) unexpectedly also found that segmentation level had an impact on well-being. 
Their showed that well-being was higher when segmentation supplies and values were both high as opposed to when both were low, indicating that overall, level of worknonwork boundary segmentation was also a predictor of well-being. These findings indicate that wanting and attaining a high degree of segmentation between work and family may be better for well-being than wanting and attaining a high degree of integration between work and family (Edwards \& Rothbard, 1999). Similarly, Kossek, Lautsch, and Eaton (2006) found that highly segmented boundaries were related to lower levels of depression, although the effects did not reach statistical significance.

High levels of segmentation appear to be beneficial for work-nonwork conflict as well. In his study of work-nonwork fit and work-home conflict, Kreiner (2006) also unexpectedly found significant main effects of segmentation level in addition to effects of work-nonwork boundary fit. As mentioned previously, results showed that as segmentation supplies increased towards preferences, work-home conflict decreased. However, results showed that work-home conflict continued to decrease as segmentation supplies surpassed preferences. This suggests that there exists a main effect of segmentation level, such that in general, highly segmented environments may protect workers from work-home conflict. Similarly, Kossek and colleagues (2006) found that individuals with highly integrated boundary management strategies had higher levels of work-nonwork conflict, especially in the nonwork-to-work direction.

This pattern regarding the importance of segmentation level seems to hold true for the outcome of instrumental work-nonwork enhancement as well. The study conducted by Chen and colleagues (2009), similar to other studies mentioned here, focused on the 
effects of work-nonwork boundary fit, but also found results that indicated that the level of boundary segmentation had a simple effect on instrumental work-nonwork enhancement. Specifically, the study found that higher levels of segmentation lead to more instrumental work-family positive spillover.

In short, it appears that the overall level of segmentation present in a particular boundary may also be a predictor of the outcomes examined in this proposed dissertation. But why might this be the case? Each of the studies cited here were theoretically grounded in work-family border theory and boundary theory, which state that there is no single "right" way to manage the boundaries between domains. Specifically, Ashforth and colleagues (2000) posit that there are both advantages and disadvantages to each type of boundary management style. For individuals with highly segmented boundaries, the transitions between roles are effortful and involve many rites, but reduce any sort of confusion about roles. In contrast, individuals with highly integrated boundaries have transitions between roles that are much easier and involve fewer rites, but this ease comes at the cost of increased blurring between roles and uncertainty (Ashforth et al., 2000). Individuals with highly integrated work-nonwork boundaries may make these role transitions almost constantly throughout the day, while individuals with highly segmented work-nonwork boundaries only transition twice a day-once as they go to work and again as they leave work (Ashforth et al., 2000). Role boundaries with high levels of flexibility and permeability allow for more frequent role transitions. Highly integrated roles have far more blurring of roles, which may foster confusion about which role should be the most salient and interruptions that disrupt functioning (Ashforth et al., 
2000). The high level of role integration may result in increased levels of cognitive complexity, subsequently resulting in higher work-nonwork conflict, frustration, and depression (Kossek et al., 2006). For example, Kossek and colleagues (2006) found that individuals with highly integrated boundary management strategies have significantly higher family-to-work conflict, and slightly higher work-to-family conflict and depression levels. Kossek and colleagues (2006) concluded that boundary management strategies that favor separation of work and nonwork are strong positive predictors of individual well-being.

The answer to why high levels of boundary segmentation seem beneficial for individual outcomes may lie in the literature on the topic of recovery from the workplace. Recovery, the process during which individual functional systems that have been drained during a stressful experience return to their prestressor levels, can protect health (Sonnentag \& Fritz, 2007). Four types of recovery experiences have been proposed. In order to recover from the demands of the workplace, individuals may 1) psychologically detach from work (not just physically distance oneself from work), 2) relax in a state of low activation and increased positive affect, 3 ) engage in mastery experiences, or learning opportunities in domains outside of work, and/or 4) exert control over leisure time in choosing which activities to pursue and when to pursue them (Sonnentag \& Fritz, 2007). High levels of job stressors, such as a heavy workload or emotional dissonance, create a need for recovery (Sonnentag, Kuttler, \& Fritz, 2010). Individuals who engage in emotion-focused coping are more likely to engage in these recovery strategies as a way to cope with negative situations at work. The ability to recover from work has an impact 
on health and well-being. For example, Sonnentag and Fritz (2007) found that recovery experiences, especially psychological detachment, predicted psychological wellbeing.

It is easy to draw parallels from the recovery literature to the work-nonwork boundary literature; highly segmented roles allow psychological detachment and thus, can lower work-nonwork conflict and improve health. Recent research supports the idea that a relationship does exist between work-nonwork boundary segmentation level and psychological detachment. Specifically, Park, Fritz, and Jex (2011) found that individuals with a stronger preference for segmenting the work and nonwork domain (rather than integrating the two) had higher levels of psychological detachment from work.

Thus, it appears that although theory does not specifically state that highly segmented boundary management strategies are more beneficial, previous empirical results and other related theory from other areas of OHP seem to indicate that individuals with highly segmented boundaries are likely to have more positive outcomes than those who choose to integrate their domains. It is worth noting that this idea of segmentation level as a predictor of desirable outcomes is complementary to, rather than conflicting with, the theory of work-nonwork boundary fit. Both level of boundary segmentation and congruence between individual segmentation preferences and organizational segmentation supplies can influence outcomes. The most optimal outcomes are produced when an individual wants a high level of segmentation and is able to achieve that level of segmentation (e.g., Chen et al., 2009; Kossek et al., 2006; Kreiner, 2006). 
Due to the relative lack of theoretical underpinning, this supposition is not presented as a hypothesis, but rather as a research question. Specifically, Research Question: Are segmented boundary management strategies better for the outcomes of work-nonwork conflict, work-nonwork enhancement, and health than integrated boundary management strategies? 


\section{Chapter 4}

\section{Methods}

\section{Overview of Study Design}

This study utilized an online survey method to examine work-nonwork boundary fit in relation to health. The survey was approximately 15 minutes long, confidential in nature, and was incentivized with a lottery-style chance of winning one of four $\$ 50$ Visa gift cards. A two-wave design was utilized in order to strengthen causal inferences; a three-month time lag separated measurement periods. More details and the rationale behind each of these choices are detailed below.

\section{Sample and Participants}

Time 1 Recruitment and Data Collection. Initial participants for the present study were recruited using a snowball sampling approach (Scott, 1991). In this approach, I sent out initial recruitment requests via email (see Appendix A for the full text of the recruitment materials for Time 1). I then asked others to pass the recruitment posting on to their contacts that might be willing to participate in the survey. This widespread recruitment technique may have resulted in a very high non-response rate, although it is difficult to estimate. However, the technique also allowed for recruitment of a diverse sample from a variety of occupations, organizations, and geographic locations. Additionally, this methodology was able to reach participants quickly, enabling the project to move forward in a timely manner.

The recruitment email included details about the study, the purpose, and the researchers. Additionally, it described the inclusion criterion: the survey was only open 
to individuals who worked 20 hours or more per week. The purpose of this inclusion criterion was to obtain a sample that had significant roles in both the work and nonwork domains, thus increasing the possibility of role conflict and enhancement. Unlike many traditional work-family studies, there was no inclusion criterion regarding the nonwork role. While previous research has indicated that certain home role variables can impact work-nonwork conflict and work-nonwork enhancement, such as marital status or the presence and number of children living in the home, this study sought to examine the broader construct of work-nonwork conflict and work-nonwork enhancement, thus including individuals that did not have children and those that were not married.

In the hopes of increasing participation, the recruitment email also described the incentive: by participating in the study and entering their email address on the final page of the survey, participants were automatically entered in a lottery-style drawing to win one of four $\$ 50$ Visa gift cards. Visa gift cards were strategically chosen as an incentive, as it enabled the winners to purchase a multitude of items and therefore was appealing to many types of potential participants. Winners were notified at the conclusion of the study in September of 2012.

As an additional incentive, participants were informed that they could receive a copy of the results upon completion of the study if they were so inclined. Those who requested a copy of the results were promised an e-pamphlet of major findings once the study is concluded. This opportunity for additional study information was intended to increase the participants' feeling of ownership in the survey, and thereby increase their response rates at Time 2. See Appendix B and Appendix C for the text of this offer (in 
the introduction to the survey and on the closing "thank you" page of the survey at both time points).

Recruitment for Time 1 data collection began on January $17^{\text {th }}, 2012$ and ended on March $21^{\text {st }}, 2012$. Over the course of these nine weeks, I sent 102 recruitment emails or messages to my social contacts (see Appendix A for the full text of the recruitment email). Additionally, I sent recruitment emails out via two listservs: the Portland Industrial Organizational Psychology Association (PIOPA, approximately 103 members at the time) and the Psychology Graduate Student Association at Portland State (PGSA, approximately 75 members at the time). I also sent an email and a reminder one week later to the OMSI All Staff email list (approximately 267 individuals). Thus, via email, approximately 547 individuals received the recruitment email directly from me.

Unfortunately, there is no way to calculate the number of individuals who passed it on, and thus, response rate for Time 1 data collection cannot be calculated.

I also utilized social networking sites to recruit participants. I posted the recruitment on Facebook three times over the course of the nine weeks, and, to my knowledge, it was reposted by at least seven individuals. I also posted the recruitment on my Google + page, and it was reposted at least once that I know of (see Appendix A for the full text of the recruitment posting for social networking sites).

Lastly, the recruitment was posted on two online forums on About.com. The first was a thread in the psychology forum, entitled "Requests for Research Participants" (available online at http://forums.about.com/n/pfx/forum.aspx ?folderId=5\&listMode=13\&nav=messages \&we 
btag=ab-psychology). The second was a thread in the parenting forum, entitled "Working Moms" (available online at http://forums.about.com/n/pfx/forum.aspx?nav=messages\&webtag=ab-workingmoms). See Appendix A for the full text of the recruitment posting. The recruitment in the "Request for Research Participants" thread was viewed 65 times, while the recruitment on the "Working Moms" thread was viewed 11 times.

A power analysis extrapolating response rates in previous studies was used to calculate a sufficient sample size for Time 1 data collection. The power analysis was conducted by a Monte Carlo simulation using Mplus software. Inputs for the Monte Carlo simulation consisted of correlations from previously published studies on the various constructs, where at least one measure in each correlation was identical to the measures proposed for use in this dissertation. The Monte Carlo analysis was conducted for 10,000 iterations using an alpha level of .05. Results indicated that a sample size of 300 participants would lead to an approximate power level of .74 in assessing the indirect effect of congruence on health through the mechanism of work-nonwork conflict. A second simulation showed that for the indirect effect of work-nonwork boundary fit on health and well-being through work-nonwork enhancement, a sample size of 300 would result in an approximate power of .89 , provided measures of work-nonwork enhancement were instrumental in nature. Thus, it appears that a final sample of 300 participants (with data at both time points) would provide adequate power to reject the null hypothesis when it is indeed truly false. 
This final target sample size was combined with statistics on the average response rate for online organizational surveys distributed via email to calculate the target Time 1 sample size (55\%; Baruch \& Holtom, 2008). Thus, I determined that Time 1 data collection could end when at least 600 participants (with email addresses entered) had completed the survey. The nine weeks of data collection garnered 749 participants, 603 of which chose to enter their email addresses for re-contact at Time 2. Thus, the sample size was deemed sufficient at that time, and data collection for Time 1 was closed.

Time 2 Recruitment and Data Collection. All Time 1 participants who chose to include their email address when completing the survey were emailed a recruitment letter at Time $2(n=603)$. The Time 2 recruitment email was very similar to the Time 1 recruitment email; it included details about the study, the purpose, and the researchers (see Appendix D for the Time 2 recruitment materials). Participants were also informed that by participating in the second survey, they would be entered a second time in the drawing for one of four $\$ 50$ Visa gift cards. It was reiterated that they could contact the primary researcher if they wanted to receive a copy of the major findings once the study was completed.

Time 2 data collection began on April $17^{\text {th }}, 2012$, and closed on June $11^{\text {th }}, 2012$. The recruitment was strategically designed to contact each individual three months after they had completed the Time 1 survey. As mentioned previously, Time 1 participants had completed the data at various times over the course of nine weeks. Thus, to make sure all participants were recruited for the Time 2 survey three months after their original survey, Time 2 recruitment was conducted over another nine weeks. To have an exact 
three-month time lag between surveys, it would have been necessary to send out recruitment emails every day over the course of those nine weeks. To simplify this process while preserving the three-month time lag, the participants were grouped into nine separate recruitment campaigns, organized by the date at which they had completed their Time 1 survey. Thus, each week, a recruitment email went out to the group of individuals who had completed their initial Time 1 survey three months prior (plus or minus seven days). A reminder email was sent to them three days after the initial recruitment, and then once again five days after the first reminder (see Appendix D for the text of these reminder emails). This campaign format was utilized each week in order to reach all of the Time 1 participants after a time lag of three months (plus or minus seven days), regardless of when they had completed the first survey.

A total of 354 participants completed the Time 2 survey. Given that 603 individuals were recruited, this indicates a response rate of $58.71 \%$. It is worth noting, however, that several individuals mentioned to me personally that the message had been sent to their spam folder. This is likely due to the fact that the Time 2 campaigns were sent by SurveyMonkey (although they were sent on behalf of a pdx.edu account). It is impossible to know how many of these recruitment emails were sent to spam, and thus were not seen by the potential participants. Therefore, this response rate may be conservative. Previous research indicated that the average response rate for organizational surveys ranged from $38.9 \%$ (web distribution) to $62.4 \%$ (in person distribution), and that response rates for emailed surveys had an average response rate of 54.7\% (Baruch \& Holtom, 2008). Additionally, Ployhart and Vandenberg (2010) stated 
that it is not uncommon for response rates to drop in excess of $50 \%$ between time points. Thus, by these standards, a response rate of $58.71 \%$ is acceptable.

\section{Instrument}

I chose to utilize online surveys for strategic purposes. Admittedly, the method of recruitment for these online surveys is not random, and the lack of random sampling limits the generalizability of the results. There is also a possibility that using online surveys may have created a non-responder difference, as it is possible that individuals who responded are more educated or tech-savvy than those who did not respond. However, I feel that the advantages of using an online survey outweigh the disadvantages. Using an online survey enabled me to widely distribute the list for very little cost, allowing me to reach a wide variety of participants from diverse organizations and occupations with a greater geographic dispersion. Additionally, using an online survey allowed me to personalize recruitment emails and send automatic reminders, which likely increased the response rate, providing greater confidence in results.

The Time 1 survey contained 79 items and seven additional opportunities to give open-ended feedback. The Time 2 survey contained 70 items and seven additional opportunities for open-ended feedback. Most participants completed the survey in 15 to 20 minutes. All scales were included on both surveys, in order to enable investigation of the question of reverse causality (Stone-Romero, 2011). See Appendix B for the Time 1 survey and Appendix $\mathrm{C}$ for the Time 2 survey. 
In order to make causal inferences, temporal precedence is required. Thus, in the present study, data collection was conducted twice, with a three-month time lag between collection points. Ideally, the time lag between surveys would be theoretically grounded, but in reality, very few theories include precise specification of time intervals for changing phenomena (Mitchell \& James, 2001). The theories examined in this study are no exception. Thus, a more general search of the literature was conducted to justify the selection of the three-month time lag.

The hypotheses proposed in the current study are based on the stressor-strain conceptualization of health. According to this theory, stressors are events, characteristics, or situations that create stress. In contrast, strain is the individual's psychological and/or physiological response to stress (Hurrell, Nelson, \& Simmons, 1998). Cognitive-relational theory of stress states that two factors mediate the relationship between stressors and strain: appraisal and coping (Hart \& Cooper, 2001). Appraisal is a cognitive process whereby the individual assesses his or her environment and judges whether the conditions are likely to influence their well-being. Coping processes can then be enacted to alter their environment or their outcomes. These coping efforts can either reduce or increase strain, depending on the effectiveness of the selected coping method (Edwards, 1992; Lazarus, 1990). Psychological and physical symptoms arise as a result of depleted coping resources (Hockey, 1993; Melamed, Shirom, Toker, Berliner, \& Shapira, 2006). Based on this research, it is logical that an individual that appraised a stressor as a threat to his or her well-being would have an initial negative health reaction (e.g., increased adrenaline, feelings of panic). The individual would 
likely then attempt to cope with said stressor. If these efforts are unsuccessful in reducing or eliminating the stressor, the individual may invest even more coping resources, thus creating a depletion of said coping resources, and leading to psychological or physical symptoms (Darr \& Johns, 2008). In this example, it is clear that this process might take several weeks or months to use up coping resources and for symptoms to start to appear. Thus, a three-month time lag appears to be a reasonable amount of time to allow said symptoms to occur.

An examination of the field of published stress research demonstrated that a threemonth time lag was well within the norm for similar studies. Specifically, previous research examining time lags in longitudinal health studies found that time lags in published studies ranged from 1 month to 10 years or more, and the majority of time lags were 1 year or less (Zapf et al., 1996). Additionally, the outcome measure utilized in this study, the SF-12, has been designed to assess health on a monthly basis, and so it is sensitive enough to measure health changes over a three-month time lag (McDowell, 2006).

The survey was confidential in design, as email addresses were collected from participants (although they were not required, and participants could participate without entering their email address). Email addresses were removed prior to analyses, so the data was analyzed in a truly anonymous manner.

\section{Measures}

Predictor measures. Boundary fit was measured using the scale created by Kreiner (2006) for use in a very similar study. The scale consists of two sub-scales, one 
for segmentation preferences (4 items) and another for segmentation supplies (i.e., characteristics of the workplace; 4 items). Each scale is measured on a 7 point Likerttype scale from strongly disagree to strongly agree. A sample item from the segmentation preferences subscale is, "I don't like to think about work while I'm at home". A parallel sample item from the segmentation supplies sub-scale is, "My workplace lets people forget about work when they're at home". Similar to how Kreiner (2006) and Chen and colleagues (2009) operationalized it, congruence was measured by complementary fit, or the degree to which the average scores on the segmentation supplies scale are similar to the average score on the segmentation preferences scale. See Appendix B and C for the full text of these scales. Cronbach's alpha was .93 for segmentation preferences and .95 for segmentation supplies, indicating strong scale reliability.

Mediator measures. Work-nonwork conflict was measured using the multidimensional, bidirectional measure of work/nonwork interference developed by Fisher and colleagues (2009). This 11-item scale was developed expressly to answer the need to expand upon existing measures that only captured work-family domains, and broaden the concept to apply to work and nonwork. The scale consists of two sub-scales: work interference with personal life (5 items, $\alpha=.89$ ) and personal life interferences with work (6 items, $\alpha=.83$ ). A sample item from the work interference with personal life sub-scale is, "I come home from work too tired to do the things I would like to do". A sample item from the personal life interference with work sub-scale is, "My personal life drains me of the energy I need to do my job". See Appendix B and C for the full text of 
each sub-scale. The scale is theoretically grounded in many of the theories used to develop the proposed study, including role theory (Kahn et al., 1964) and the attendant role interference and role accumulation theories, as well as conservation of resources theory (Hobfoll, 1989).

While the Fisher et al. (2009) scale does include a second sub-scale to measure work-nonwork enhancement, I chose not to utilize that portion of the scale. The Fisher et al. (2009) work-nonwork enhancement scale includes 1 item each to measure affective, instrumental, and energy-based enhancement. The energy-based enhancement component is not theoretically supported by previous research, and results of a Monte Carlo power analysis indicated that using a scale that measured affective work-nonwork enhancement would likely result in an underpowered study, even with sample sizes above 1,000. Thus, only one item on the scale (instrumental enhancement) is theoretically grounded and is likely to have the power to detect real differences. This was unsatisfactory, so I decided to use a larger scale to measure work-nonwork enhancement, in order to obtain multiple items with the theoretical background and sufficient power. As there are not (to my knowledge) any existing scales that meet these criteria, I chose to adapt the measure of work-nonwork enhancement created by Hanson, Hammer, and Colton (2006).

The initial measure developed by Hanson and colleagues was designed to measure both work-to-family enhancement and family-to-work enhancement over the two theoretical components, instrumental and affective. In their scale, instrumental enhancement is represented by behavior/skills and by values. I decided to include the 
affective sub-scales as well. Although the Monte Carlo power analysis indicated that it was unlikely that these affective enhancement items will have adequate power, including this sub-scale does allow for a complete picture of both of the theoretical types of enhancement, and thus is included for theoretical purposes.

In order to adapt this scale for the broader concept of work-nonwork, the word "family" in each item was changed to words like "personal life" or "personal responsibilities", in keeping with the grammatical structure of the sentence.

As the scale measures both directions of work-nonwork enhancement across all three types of enhancement (behaviors/skills, values, and affective), there are a total of six subscales. Instrumental enhancement is measured with four sub-scales: 4 items to measure behavior-based instrumental work-to-nonwork enhancement $(\alpha=.88), 3$ items to measure value-based instrumental work-to-nonwork enhancement $(\alpha=.90), 4$ items to measure behavior-based instrumental nonwork-to-work enhancement $(\alpha=.88)$, and 3 items to measure value-based instrumental nonwork-to-work enhancement $(\alpha=.92)$. Affective enhancement is measured with two additional sub-scales: 4 items to measure work-to-nonwork affective enhancement $(\alpha=.86)$ and 4 items to measure nonwork-towork affective enhancement $(\alpha=.91)$.

A sample item from the work-to-nonwork behavior sub-scale is, "Skills developed at work help me in my personal life". A sample item from the work-tononwork value sub-scale is, "Values developed at work make me a better person outside of work". A sample item from the work-to-nonwork affective sub-scale is, "When things are going well at work, my outlook regarding my personal life is improved". A sample 
item on the nonwork-to-work behavior sub-scale is, "Skills developed in my personal life help me in my job". A sample item on the nonwork-to-work values sub-scale is, "My personal values make me a better employee". A sample item on the nonwork-to-work affective sub-scale is, "When things are going well in my home life, my outlook regarding my job is improved". The full text of the adapted items is available in Appendices B and C. For the full text of the original items, see Hanson et al. (2006).

Outcome measures. Health was measured using one of the most widely used health measures available, the Short-Form-12 Health Survey (SF-12; Ware, Kosinski, \& Keller, 1996). The SF-12 is an abbreviated version of the popular SF-36 Health Form that is designed to be brief enough for practical use in large-scale surveys but still give a strong indicator of health status. The SF-12 includes items that cover physical functioning, bodily pain, vitality, social functioning, mental health, and general health, among others. There are two principle scores that stem from the SF-12: the physical health composite score (PCS-12) and a mental health composite score (MCS-12; Ware, Kosinski, Turner-Bowker, \& Gandek, 2002). These composite scores are norm-based, and are calculated by QualityMetric, the owner of the scale, as per the terms of use agreement. Previous research shows strong scale reliability for both the PCS-12 $(\alpha=$ $.81)$ and the MCS-12 ( $\alpha=.84$; Lim \& Fisher, 1999). Additionally, initial research shows that the SF-12 is resistant to both ceiling and floor effects (Bennett et al., 2002). A sample item from the PCS-12 is, "During the past 4 weeks, how much did pain interfere with your normal work (including both work outside the home and housework)?” A sample item from the MCS-12 is, "How much of the time during the past 4 weeks have 
you felt calm and peaceful?" The full text of the SF-12 can be found in Appendices B and $\mathrm{C}$.

Given that previous research has found relatively high alpha coefficients for both the entire scale and the two sub-components of the SF-12, and given that previous research has linked the predictors in this study (work-nonwork boundary fit, worknonwork conflict, and work-nonwork enhancement) to a myriad of health indicators (e.g., Eby et al., 2005), no differential predictions are made based on the outcome measure subscales. That is, no hypotheses are created related to the relative strength of the hypothesized relationships on each of the two subscales.

Control measures. In order to increase certainty that observed differences are, in fact, due to the hypothesized relationships, potential confounding variables should be controlled for during analyses. Several control variables were selected for inclusion in this study due to previous empirical research linking them to the variables of interest included in these hypotheses. All control variables can be found in the sample surveys in Appendices B and C.

Certain characteristics of work and nonwork domains have the potential to influence an individual's experience of the work-nonwork interface. This is not surprising, given that both work-nonwork conflict and work-nonwork enhancement have been shown to be cross-domain in nature (i.e., work characteristics most strongly predict work-to-nonwork conflict, while nonwork characteristics most strongly predict nonworkto-work conflict; Ford et al., 2007). For instance, characteristics of the nonwork domain, such as marital status, number of children, and the number of children living at home, 
have been empirically linked to the constructs of work-nonwork conflict and worknonwork enhancement (Hammer \& Zimmerman, 2011). Characteristics of the work domain, such as job type and the number of hours worked per week, are also related to the constructs of interest in this study (Hammer \& Zimmerman, 2011). Thus, in order to assess whether the hypothesized relationships are generalizable for workers from a broad spectrum of work environments and a broad spectrum of home environments, the following characteristics were included as control variables: number of dependents living at home, household status, job type, industry type, and number of hours worked per week.

There is some evidence that the interface between work and nonwork looks different for individuals of different ages. Generally speaking, older workers tend to experience great work-nonwork enhancement and less work-nonwork conflict than their younger counterparts (Grzywacz et al., 2002; Matthews et al., 2010). This may be due in part to socio-emotional selectivity theory (Carstensen, 1992), which states that older adults are more focused on gratifying social relationships than younger adults, who may be more focused on career goals. Thus, in order to account for potential differences in the experience of the work-nonwork interface, age was included as a control variable.

There are mixed findings regarding whether gender alters an individual's experience of the work-nonwork interface (e.g., Eby et al., 2005). Due in part to gender role expectations, women are expected to prioritize family and caretaking roles, while men are expected to prioritize work roles (Gutek, Searle, \& Klepa, 1991). Thus, it is possible that experiences of work-nonwork boundary fit, work-nonwork conflict, and 
work-nonwork enhancement may be different for men and women. In order to account for this, gender was included as a control variable.

Lastly, socioeconomic status (SES) has been demonstrated to have a strong link to health, such that individuals with lower SES are prone to diminished health and longevity (Christie \& Barling, 2009). There may be multiple reasons for this relationship, including reduced access to healthcare, poorer quality food, lesser education on correct health behaviors, less control over work, and more dangerous jobs, among others (Christie \& Barling, 2009). Thus, in order to gain more confidence that any changes in health and well-being are, in fact, due to the hypothesized relationships and not SES differences, two measures of SES were included as control variables during analyses: level of education and income.

Additional measures. For the purpose of potential post-hoc analyses, two other measures were included in the survey. The first of these was a measure of psychological detachment. This measure is one of the four subscales from the Sonnentag and Fritz (2007) recovery from work scale. The psychological detachment subscale consists of four items. Respondents are asked to describe how well each statement fits with their experience of leisure time on a 5-point Likert-type scale that ranges from "not at all" to "always". For example, one item is "I forget about work". In the current study, Cronbach's alpha was .87, indicating strong validity.

The second additional scale was intended to measure perceived personorganization fit. This scale was adapted from a three-item scale created by Cable and Judge (1996). Respondents are asked to rate their agreement with the items on a 5-point 
scale ranging from not at all to completely. An example of one of the adapted items is, "To what degree do you feel your work-life values match or fit with those of the organization?" In the current study, Cronbach's alpha was .88, indicating strong validity.

Open-ended items. Each measure (i.e., work-nonwork boundary fit, worknonwork conflict, work-nonwork enhancement, well-being, psychological detachment, person-environment fit, and demographics) was accompanied by an open-ended question for thoughts or further explanation of the responses given to the quantitative questions. Responses to these seven open-ended questions were analyzed qualitatively. These qualitative responses give context and depth of understanding to the quantitative data. This qualitative data may inform and guide future quantitative research outside the scope of the present study (Lee, Mitchell, \& Harman, 2011). 


\section{Analyses}

Rationale for Primary Analysis Technique. Similar to Chen and colleagues' (2009) study which examined work-nonwork boundary fit, work-nonwork conflict, and work-nonwork enhancement, latent congruence modeling (LCM) was used to test the hypotheses. LCM is a variation of structural equation modeling (SEM) that has recently been developed to assist in analyzing congruence issues in organizational research (Cheung, 2009).

Studying congruence concepts in organizational research is not unusual, but operationalizing the concept of congruence has posed some difficulties (Cheung, 2009). Congruence can be operationalized as a difference score directly comparing two components, such as person and environment. However, this method of analysis can result in lower reliability and a lack of precision regarding the contributions of the individual components on the outcome measures, rather than just their difference (Cheung, 2009). Another method of congruence analysis is that of polynomial regression, which does allow examination of the relationship between each individual component and the outcome variable. However, this method, like difference score analysis, assumes that components are measured without error, thus potentially producing biased estimates of the strength of relationships (Edwards, 1994).

In order to address these issues, Cheung (2009) developed a method of analyzing congruence using structural equation modeling (SEM) called latent congruence modeling, or LCM. LCM was designed specifically to examine congruence issues in organizational research. In LCM, two higher-order factors are created that represent both the mean 
(level) and difference (congruence) of the two interdependent component measures (in this case, personal boundary preferences and organizational boundary supplies). These components are first-order latent variables, as measured by multiple indicators (in this case, individual questions on each of the appropriate scales). This allows for estimation of the error terms involved with each scale, and measurement equivalence of the two components.

Primary Data Analysis. The basic steps in conducting any SEM, including LCM, are as follows: 1) specify the model, 2) evaluate model identification, 3) select measures and collect, prepare, and screen the data, 4) estimate the model, including evaluations of fit and interpretation of parameter estimates, and 5) reporting of results (Kline, 2011). The introduction of this dissertation proposal has fulfilled the first step of specification. That is, relationships are proposed based upon theory and previous empirical evidence. This provides a path model, or a model of how the latent constructs work together, as illustrated in Figure 1. When combined with a measurement piece, this will ultimately become a structural regression model used to test hypotheses.

Step two is a check of model identification. As is clear from the proposed model in Figure 1, there are no feedback loops, indicating a recursive model. As illustrated in the measures section, each measure has an adequate number of indicators (i.e., three or more). The combination of these two factors indicates that this model is over-identified.

The third step of SEM is to select measures and then collect, prepare, and screen the data. As described in the methods section, measures were chosen with great care and data was collected at two time points. 
Basic data cleaning was conducted on the Time 1 dataset first. All responses that did not include an email address were discarded due to the inability to collect Time 2 data $(n=146)$. All individuals who did not answer at least $35 \%$ of the questions (or 25/70, e.g., those who made it past the first two pages) were removed ( $n=111)$. The "number of hours worked per week" was cleaned as follows: 1) if individuals put a range (e.g., “40-50”), it was averaged (e.g., “45”), 2) if individuals put a plus sign (e.g., “40+”), I translated it to the next highest whole number (e.g., "41"). All individuals who entered a number of hours that was smaller than $20(n=2)$ were removed in an attempt to sample only those who had significant work responsibilities. One individual was removed for obviously untrue answers (e.g., 123 children, 123 hours per week worked).

Data cleaning on the Time 2 dataset was conducted next. All of the above data cleaning assumptions were also applied to the Time 2 dataset. A total of seven participants were removed due to not completing at least $35 \%$ of the questions. In addition, individuals who indicated they had changed jobs during the time lag were removed $(n=4)$, as were those who were unemployed $(n=1)$, and those who had been on extended vacation for the prior four weeks $(n=1$, due to the fact that four weeks is the frame of reference for several scales).

The two datasets were then merged, and any individuals who did not have Time 2 data were removed $(n=229)$. This resulted in a cleaned dataset with 339 participants, all of whom had data at both time-points.

Next, scale scores were created for the variables of interest by calculating mean scores. The exception to this is the health outcome scores. As per the usage terms of the 
SF-12 agreement, the 12 items of the health outcome scored were scored automatically using QualityMetric software. This norm-based scoring created a single item for the PCS and another single item for the MCS score. When scoring the SF-12 data, no missing data estimation was used, resulting in PCS and MCS scores only for individuals with complete answers on all 12 items. This resulted in a loss of 24 participants' data, creating a final dataset of 315 individuals.

Next, I chose control variables to include in the final model. The potential control variables included in the dataset included gender, age, household status, number of children (broken up into the presence of children under 18, the presence of children over 18 , and the total number of children in the household), education, income, and industry. In order to choose which control variables to include in the final model, I examined the correlations between the control variables and the constructs of interest in the dataset (see Table 1). I chose to include the four control variables that were significantly related to three or more of the primary constructs of interest: age, the presence of older children, education, and income. It is worth noting that the presence of young children, the presence of older children, and the total number of children were all correlated at over .85 , so it is intuitively possible to simply choose one to represent the larger construct of "children". I chose older children, as it had the most statistically significant relationships with the constructs of interest. Lastly, since the remaining control variables covaried significantly with the chosen control variables, I deemed it acceptable to only include the four control variables in an attempt at parsimony. 
Table 1. Correlation Table

\begin{tabular}{|c|c|c|c|c|c|c|c|c|c|c|c|c|c|c|}
\hline & Measure & $N$ & Min & $\operatorname{Max}$ & $M$ & $S D$ & 1 & 2 & 3 & 4 & 5 & 6 & 7 & 8 \\
\hline 1 & Boundary Prefs w1 & 315 & 1.00 & 7.00 & 5.39 & 1.42 & $(.93)$ & & & & & & & \\
\hline 2 & Boundary Supplies w1 & 315 & 1.00 & 7.00 & 3.59 & 1.67 & $.161 * *$ & $(.95)$ & & & & & & \\
\hline 3 & WtN Conflict w1 & 315 & 1.00 & 5.00 & 2.85 & 0.86 & .077 & $-.493 * *$ & $(.89)$ & & & & & \\
\hline 4 & NtW Conflict w1 & 315 & 1.00 & 3.67 & 1.97 & 0.61 & -.005 & -.061 & $.130 *$ & $(.84)$ & & & & \\
\hline 5 & Enhance_WtN_Beh_w1 & 315 & 1.00 & 5.00 & 3.55 & 0.75 & $-.239 * *$ & .022 & -.042 & .008 & $(.88)$ & & & \\
\hline 6 & Enhance_WtN_Val_w1 & 315 & 1.00 & 5.00 & 3.56 & 0.83 & $-.232 * *$ & -.005 & -.066 & .014 & $.596^{* *}$ & $(.90)$ & & \\
\hline 7 & Enhance_WtN_Aff_w1 & 314 & 1.75 & 5.00 & 4.18 & 0.59 & -.03 & -.036 & .103 & -.035 & $.175^{* *}$ & $.249 * *$ & $(.87)$ & \\
\hline 8 & Enhance_NtW_Beh_w1 & 313 & 1.00 & 5.00 & 4.00 & 0.62 & -.045 & -.097 & .039 & $-.149 * *$ & $.404 * *$ & $.256^{* *}$ & $.215 * *$ & $(.88)$ \\
\hline 9 & Enhance_NtW_Val_w1 & 314 & 2.33 & 5.00 & 4.48 & 0.55 & -.062 & $-.118^{*}$ & $.121 *$ & $-.151 * *$ & $.213^{* *}$ & $.199 * *$ & $.303 * *$ & $.390 * *$ \\
\hline 10 & Enhance_NtW_Aff_w1 & 314 & 2.00 & 5.00 & 4.02 & 0.63 & -.103 & .024 & -.036 & .052 & $.285^{* *}$ & $.293 * *$ & $.513 * *$ & $.297 * *$ \\
\hline 11 & Survey Source w1 & 315 & 0.00 & 7.00 & 2.31 & 1.63 & .035 & .063 & -.043 & .018 & .002 & .042 & $-.146 * *$ & .015 \\
\hline 12 & Gender w1 & 314 & 1.00 & 2.00 & 1.30 & 0.44 & -.093 & .016 & -.025 & -.055 & -.09 & -.038 & -.006 & -.051 \\
\hline 13 & Age w1 & 308 & 22.00 & 75.00 & 40.31 & 12.19 & -.073 & -.041 & -.025 & $-.131 *$ & .096 & .083 & -.025 & $.135^{*}$ \\
\hline 14 & Household w1 & 310 & 1.00 & 3.00 & 1.46 & 0.82 & -.003 & .056 & -.051 & .036 & .037 & .045 & -.052 & $-.118^{*}$ \\
\hline 15 & Children <18 w1 & 310 & 0.00 & 6.00 & 0.60 & 0.95 & -.088 & -.080 & .013 & $.170 * *$ & .038 & .010 & -.061 & .052 \\
\hline 16 & Children >18 w1 & 300 & 0.00 & 3.00 & 0.13 & 0.44 & .043 & -.048 & -.03 & -.034 & .094 & $.121 *$ & .031 & .093 \\
\hline 17 & Total children w1 & 299 & 1.00 & 20.00 & 13.57 & 4.17 & -.049 & -.113 & .022 & $.160 * *$ & .073 & .059 & -.042 & .083 \\
\hline 18 & Industry w1 & 311 & 1.00 & 20.00 & 13.57 & 4.17 & .067 & $-.157 * *$ & $.137 *$ & .069 & .049 & .005 & -.061 & .066 \\
\hline 19 & Education w1 & 315 & 1.00 & 8.00 & 6.99 & 1.36 & -.008 & $-.126^{*}$ & .023 & .079 & .042 & -.036 & .040 & -.020 \\
\hline 20 & Physical Health w2 & 315 & 28.87 & 69.00 & 55.89 & 6.81 & -.01 & $-.147 * *$ & .013 & .021 & .049 & .040 & $.144 *$ & .059 \\
\hline 21 & Mental Health w2 & 315 & 17.47 & 65.87 & 47.19 & 9.1 & $-.130 *$ & $.248 * *$ & $-.398 * *$ & $-.345^{* *}$ & .057 & .030 & -.044 & .030 \\
\hline 22 & Household w2 & 312 & 1.00 & 3.00 & 1.36 & 0.68 & -.021 & .047 & -.033 & .059 & -.003 & -.004 & -.097 & $-.116 *$ \\
\hline 23 & Income w2 & 309 & 1.00 & 10.00 & 6.57 & 1.93 & -.106 & $-.269 * *$ & .029 & -.023 & .068 & .049 & .054 & .093 \\
\hline
\end{tabular}

Note. "w1" indicates data collected at Wave 1, aka Time 1. "w2" indicates data collected at Wave 2, aka Time 2. "WtN" = "work-to-nonwork". "NtW" =

"nonwork-to-work". "Beh" = "behavior-based". "Val" = "value-based". "Aff" = "affective".

$* p<.05 . * * p<.01 . * * * p<.001$ 


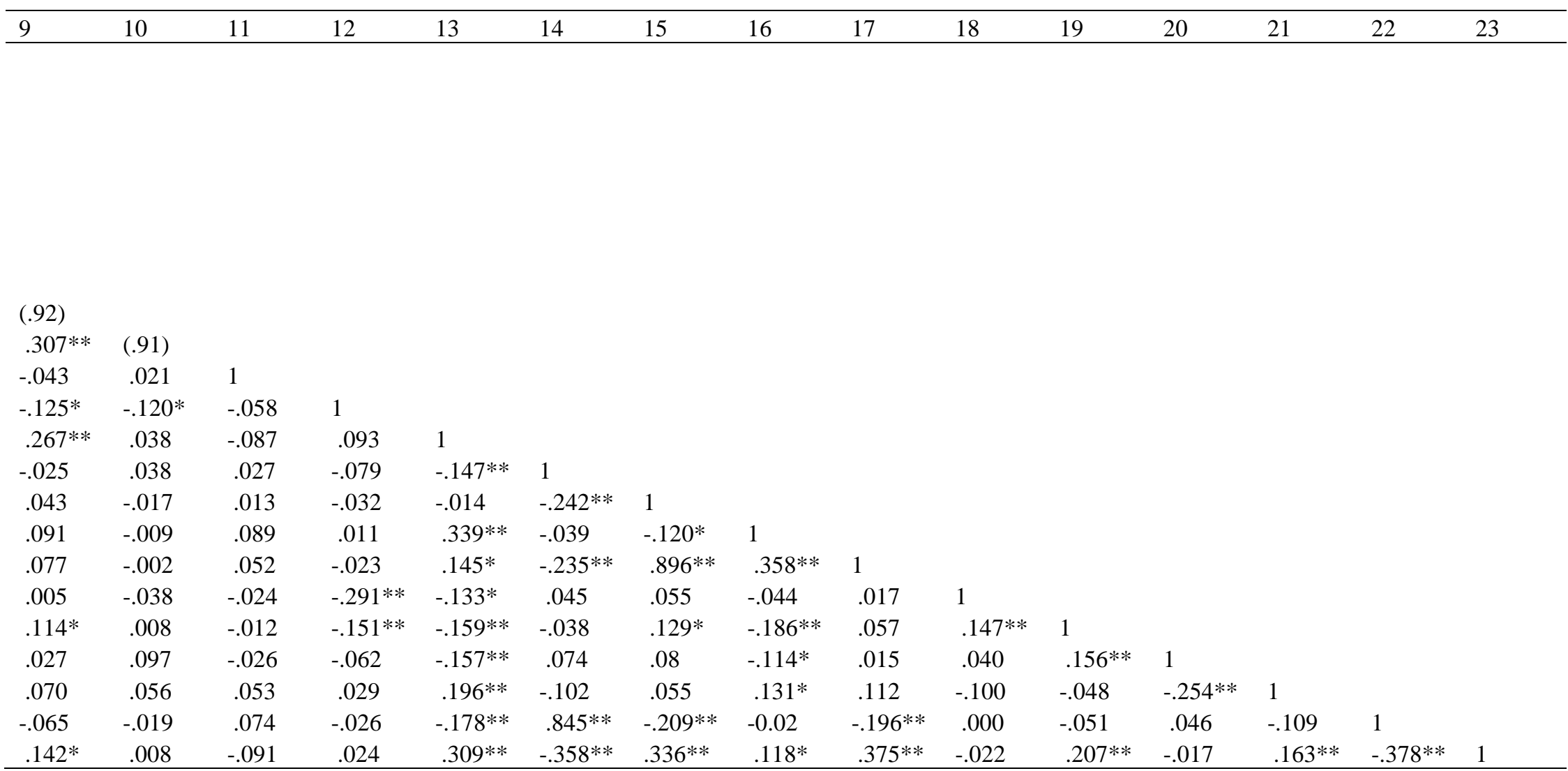

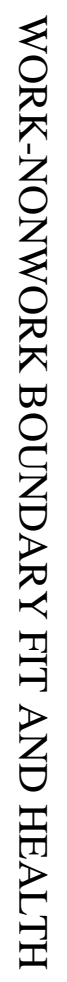


Data screening was then conducted, as per Kline (2011). To test for colinearity problems, tolerance statistics were calculated for all scale scores and the MCS and PCS. None of the tolerance values were smaller than .10, indicating no large colinearity problems. Similarly, the variance inflation factor (VIF) was calculated for all scale scores, the MCS, and the PCS. Since no VIF values were greater than 10.0, colinearity did not appear to be a problem in this dataset.

To test for outliers, the Mahalanobis distance (D) was calculated. While the Mahalanobis distance did indicate some outliers, they were determined not to be due to data entry error or from a different population (e.g., all outliers fell within the proscribed 7-point scale of an item). Thus, these outliers were retained.

To test for multivariate normality, individual univariate distributions were examined for normality. Skew and kurtosis indices were examined for all scale scores, the MCS, and the PCS. None had skew indices with an absolute value above 3.0, and none had kurtosis indices with absolute values of above 10.0, indicating univariate normality. To further test for normality, stem-and-leaf plots, box plots, and bivariate scatterplots were examined to normality. Some of these indicated slight non-normality problems.

Once the data was cleaned and screened, confirmatory factor analyses (CFAs) were conducted on all of the scale scores. The purpose of this step was to examine the measurement portion of the LCM for adequate fit (as versus the path analysis portion from Figure 1). See Appendix E for the standardized models of these CFAs and their fit statistics. All CFAs demonstrated adequate fit, with relatively strong factor loadings. 
Additionally, all the Cronbach's alphas indicated high internal consistency reliability for the scales, giving greater confidence to the usage of the scales. See Table 1 (correlation table) for more information regarding the alphas.

Due to the indications of the stem-and-leaf plots, box plots, and bivariate scatterplots that some scales may be non-normal, these CFAs were all conducted a second time utilizing a bootstrapping method with 1,000 iterations. The fit indices did not change significantly, indicating that the data appeared to be normal enough for normal estimation techniques going forward. See Appendix F for a summary of the fit statistics of each scale score utilizing bootstrapping. The fact that these two sets of CFAs (with bootstrapping and without bootstrapping) all had adequate fit indicated that it was appropriate to move on to test the fit of the entire regression model.

The full structural regression model was created using the statistical software Amos. See Figure 2 for the model as it appears in Amos. 
Figure 2. Latent Congruence Model

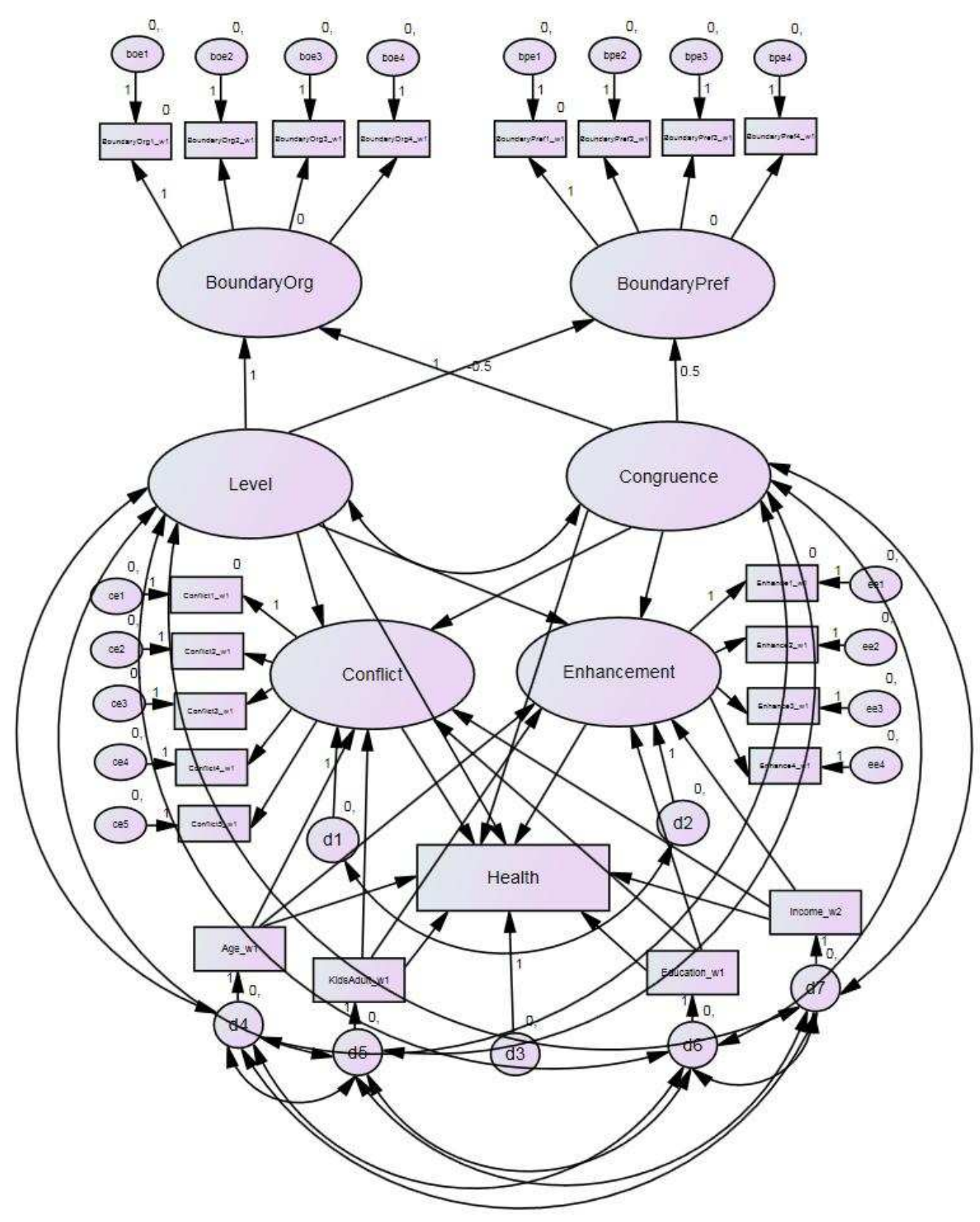

In order to properly "read" the model presented in Figure 2, the reader must be aware of the tenets of SEM. Rectangles denote observed variables, while circles or ovals 
denote latent variables. Arrows represent directed arcs, or hypothesized relationships. Double-headed arrows represent non-directed arcs, or covariances.

As illustrated in Figure 2, the latent variables "Boundary Preferences" and "Boundary Supplies" were each measured by four observed variables (the four items for each of the scales). Error terms also contributed to each of the individual scores. Then, a second-order latent variable structure was imposed ("Level" and "Congruence", as per Cheung, 2009). "Congruence", the variable of interest, was then connected to "Conflict", "Enhancement", and "Health" individually via directed paths (note that the construct of "Level" also has directed paths to each of these constructs in order to obtain adequate model fit, but since the pathways are not pertinent to the hypotheses, these results will not be discussed). The latent constructs of "Conflict" and "Enhancement" are each measured by a series of observed items (the items on the respective scales), and each also have directed paths to the outcome of interest, "Health", as per Hypotheses 1 and 2. The control variables (age, income, education, and presence of children) all have directed paths connecting them to "Health", "Conflict", and "Enhancement", as per the theoretically-based and empirically shown links discussed in the introduction section. Additionally, the disturbance terms of these control variables all covary with each other and with the other strictly exogenous variables in the model ("Level" and "Congruence"), as per structural equation modeling conventions.

Twenty-four models were necessary to test all possible combinations of the two types of conflict (work-to-nonwork and nonwork-to-work), the six types of enhancement (work-to-nonwork behavior-based, work-to-nonwork value-based, work-to-nonwork 
affective, nonwork-to-work behavior-based, nonwork-to-work value-based, and nonwork-to-work affective), and the two health outcomes (physical and mental; i.e., 2 x 6 x 2 =24). All 24 models were recursive, with a sample size of 315 . See 2 for a list of models.

Table 2. List of Models

\begin{tabular}{|c|c|c|c|c|c|}
\hline \# & Predictor & $\begin{array}{l}\text { Conflict } \\
\text { Mediator }\end{array}$ & $\begin{array}{l}\text { Enhancement } \\
\text { Mediator }\end{array}$ & $\begin{array}{c}\text { Health } \\
\text { Outcome }\end{array}$ & $\begin{array}{c}\text { Relevant } \\
\text { Hypotheses }\end{array}$ \\
\hline 1 & Congruence & WtN Conflict & WtN Behavior Enhance & Mental Health & 1a, 2a, 3a, 4a, 5a \\
\hline 2 & Congruence & NtW Conflict & WtN Behavior Enhance & Mental Health & $1 b, 2 a, 3 a, 4 b, 5 a$ \\
\hline 3 & Congruence & NtW Conflict & WtN Behavior Enhance & Physical Health & $1 b, 2 a, 3 b, 4 b, 5 a$ \\
\hline 4 & Congruence & WtN Conflict & WtN Behavior Enhance & Physical Health & $1 \mathrm{a}, 2 \mathrm{a}, 3 \mathrm{~b}, 4 \mathrm{a}, 5 \mathrm{a}$ \\
\hline 5 & Congruence & WtN Conflict & WtN Affect Enhance & Physical Health & $1 \mathrm{a}, 2 \mathrm{c}, 3 \mathrm{~b}, 4 \mathrm{a}, 5 \mathrm{c}$ \\
\hline 6 & Congruence & WtN Conflict & WtN Affect Enhance & Mental Health & $1 \mathrm{a}, 2 \mathrm{c}, 3 \mathrm{a}, 4 \mathrm{a}, 5 \mathrm{c}$ \\
\hline 7 & Congruence & NtW Conflict & WtN Affect Enhance & Mental Health & $1 b, 2 c, 3 a, 4 a, 5 c$ \\
\hline 8 & Congruence & $\mathrm{NtW}$ Conflict & WtN Affect Enhance & Physical Health & $1 b, 2 c, 3 b, 4 b, 5 c$ \\
\hline 9 & Congruence & WtN Conflict & WtN Value Enhance & Mental Health & $1 \mathrm{a}, 2 \mathrm{~b}, 3 \mathrm{a}, 4 \mathrm{a}, 5 \mathrm{~b}$ \\
\hline 10 & Congruence & WtN Conflict & WtN Value Enhance & Physical Health & $1 \mathrm{a}, 2 \mathrm{~b}, 3 \mathrm{~b}, 4 \mathrm{a}, 5 \mathrm{~b}$ \\
\hline 11 & Congruence & $\mathrm{NtW}$ Conflict & WtN Value Enhance & Physical Health & $1 b, 2 b, 3 b, 4 b, 5 b$ \\
\hline 12 & Congruence & NtW Conflict & WtN Value Enhance & Mental Health & $1 b, 2 b, 3 a, 4 b, 5 b$ \\
\hline 13 & Congruence & WtN Conflict & NtW Behavior Enhance & Physical Health & $1 \mathrm{a}, 2 \mathrm{~d}, 3 \mathrm{~b}, 4 \mathrm{a}, 5 \mathrm{~d}$ \\
\hline 14 & Congruence & WtN Conflict & NtW Behavior Enhance & Mental Health & $1 \mathrm{a}, 2 \mathrm{~d}, 3 \mathrm{a}, 4 \mathrm{a}, 5 \mathrm{~d}$ \\
\hline 15 & Congruence & $\mathrm{NtW}$ Conflict & NtW Behavior Enhance & Mental Health & $1 b, 2 d, 3 a, 4 b, 5 d$ \\
\hline 16 & Congruence & NtW Conflict & NtW Behavior Enhance & Physical Health & $1 b, 2 d, 3 b, 4 b, 5 d$ \\
\hline 17 & Congruence & NtW Conflict & NtW Affect Enhance & Physical Health & $1 b, 2 f, 3 b, 4 b, 5 f$ \\
\hline 18 & Congruence & $\mathrm{NtW}$ Conflict & NtW Affect Enhance & Mental Health & $1 b, 2 f, 3 a, 4 b, 5 f$ \\
\hline 19 & Congruence & WtN Conflict & NtW Affect Enhance & Mental Health & $1 a, 2 f, 3 a, 4 a, 5 f$ \\
\hline
\end{tabular}




\begin{tabular}{rrrrrr}
20 & Congruence & WtN Conflict & NtW Affect Enhance & Physical Health & $1 \mathrm{a}, 2 \mathrm{f}, 3 \mathrm{~b}, 4 \mathrm{a}, 5 \mathrm{f}$ \\
21 & Congruence & WtN Conflict & NtW Value Enhance & Physical Health & $1 \mathrm{a}, 2 \mathrm{e}, 3 \mathrm{~b}, 4 \mathrm{a}, 5 \mathrm{e}$ \\
22 & Congruence & WtN Conflict & $\mathrm{NtW}$ Value Enhance & Mental Health & $1 \mathrm{a}, 2 \mathrm{e}, 3 \mathrm{a}, 4 \mathrm{a}, 5 \mathrm{e}$ \\
23 & Congruence & $\mathrm{NtW}$ Conflict & $\mathrm{NtW}$ Value Enhance & Mental Health & $1 \mathrm{~b}, 2 \mathrm{e}, 3 \mathrm{a}, 4 \mathrm{~b}, 5 \mathrm{e}$ \\
24 & Congruence & $\mathrm{NtW}$ Conflict & $\mathrm{NtW}$ Value Enhance & Physical Health & $1 \mathrm{~b}, 2 \mathrm{e}, 3 \mathrm{~b}, 4 \mathrm{~b}, 5 \mathrm{e}$ \\
\hline Note. & "WtN" = "Work-to-Nonwork". "NtW" = "Nonwork-to-Work". "Enhance" = "Enhancement".
\end{tabular}

For each of these 24 models, first fit was assessed to ascertain whether there was a good fit of the model with the data, and whether it was appropriate to interpret paths. See Appendix $\mathrm{G}$ for the fit indices of each model. Once it was determined that the model fit the data well, interpretations of the specified paths was used to evaluate the hypotheses. Hypothesis 1 was assessed by examining the path coefficient between "Conflict" and "Health" in each of the 24 models. Hypothesis 2 was assessed by examining the path coefficient between "Enhancement" and "Health" each of the 24 models. Hypothesis 3 was assessed by examining the path coefficient between "Congruence" and "Health" in each of the 24 models.

Since Hypotheses 4 and 5 proposed indirect effects, it is not enough to simply examine the path coefficients between the variables of interest. Instead, the path coefficients must be used as input for the Sobel test to assess significant indirect effects (Sobel, 1986). First, the indirect effect must be calculated. For Hypothesis 4, this is found by multiplying the path coefficient from the path between the latent variables "Congruence" and "Conflict" with the path coefficient from the path between the "Conflict" and "Health". Similarly, for Hypothesis 5, the total indirect effect will be calculated by multiplying the path coefficient from the path between "Congruence" and 
"Enhancement" with the path coefficient from the path between "Enhancement" and "Health". In order to test the significance of these indirect effects, the approximate standard error for these indirect effects was calculated using the formula:

$$
S E_{a b}=\mathfrak{v b}^{2} S E_{a}^{2}+a^{2} S E_{b}^{2}
$$

Where $a$ is the unstandardized coefficient for the path $\mathrm{X}$ to $\mathrm{Y} 1$ and $S E_{a}$ is its standard error, and $b$ is the unstandardized coefficient for the path $\mathrm{Y} 1$ to $\mathrm{Y} 2$ and $S E_{b}$ is its standard error. Thus, in Hypothesis 4, $a$ represents the path from congruence to conflict, $S E_{a}$ is the standard error of congruence and conflict, $b$ represents the path from conflict to health, and $S E_{b}$ is the standard error of conflict and health. Similarly, for Hypothesis 5, $a$ represents the path from congruence to enhancement, $S E_{a}$ is the standard error of congruence and enhancement, $b$ represents the path from enhancement to health, and $S E_{b}$ is the standard error of enhancement and health.

Once this standard error was calculated for both Hypothesis 4 and Hypothesis 5, the ratio $a b / S E_{a b}$ was interpreted as the $\mathrm{z}$ test of the unstandardized indirect effect, where $p<.05$ was considered support for each hypothesis. The final step of SEM, reporting, will be fulfilled via the dissertation defense and any subsequent publications or presentations.

Secondary analysis. One drawback to using LCM to analyze congruence data is that the technique does not allow for examination of any potential asymmetry in fit, as posed in the research question. In other words, outcomes may be different depending on the direction of the misfit. For instance, does health suffer equally when supplies exceed preferences as when supplies do not meet preferences? LCM cannot answer these 
questions of the potential differential impact of types of asymmetry. Thus, response surface mapping was used to analyze polynomial regression analyses to answer the research question.

The variables Boundary Preferences and Boundary Supplies were each mean centered, and then used to create several second-order terms: Preference ${ }^{2}$, Preference $*$ Supplies, and Supplies ${ }^{2}$. These new terms, along with the centered Boundary Preference and Boundary Supplies, were used as predictors in a series of linear regressions predicting the outcomes of interest (i.e., the two types of health, two types of conflict, and six types of enhancement). The coefficients produced by this output were then added to the response surface mapping spreadsheet provided by Dr. Edwards (available online at http://public.kenan-flagler.unc.edu/faculty/edwardsj/downloads.htm). This calculation produced response surface maps and corresponding output.

These graphs were then assessed visually, and then the significance of the slopes of the $S=P$ (i.e., supplies $=$ preferences $)$ and $S=-P$ (i.e., supplies $=-$ preferences $)$ lines were assessed to determine whether there was a significant difference between levels of fit and misfit respectively. The significance of these slopes was assessed using F-tests of the coefficients in the moderated polynomial regression, as per Edwards (2002) and Edwards and Parry (1993). Syntax pertaining to these F-tests can be found on Dr. Edwards' website, http://public.kenanflagler.unc.edu/faculty/edwardsj/SPSSResponseSurfaceAnalysis.htm.

Qualitative Analysis. Each one of the survey pages contained an open-ended text box that invited participants to share any additional detail they thought needed to 
understand their numeric responses. Thus, at each time point individuals were offered seven opportunities to give written feedback (one on the boundary fit page, one on the work-nonwork conflict page, one on the work-nonwork enhancement page, one on the health page, one on the psychological detachment section, one on the P-O fit section, and a final one for overall comments). In the final dataset, there were a total of 782 responses (See Table 3 for the breakdown by individual section).

Table 3. Number of Open-Ended Responses

\begin{tabular}{lr}
\hline Section & Number of Responses \\
\hline Boundary Fit w1 & 139 \\
Work-Nonwork Conflict w1 & 61 \\
Work-Nonwork Enhancement w1 & 37 \\
Health w1 & 64 \\
Psychological Detachment w1 & 43 \\
P-O Fit w1 & 51 \\
Final w1 & 39 \\
Boundary Fit w2 & 107 \\
Work-Nonwork Conflict w2 & 50 \\
Work-Nonwork Enhancement w2 & 26 \\
Health w2 & 58 \\
Psychological Detachment w2 & 39 \\
P-O Fit w2 & 45 \\
Final w2 & 23 \\
\hline Total & 782 \\
\hline Note. "w1" = "Wave 1", the data collected at Time 1. "w2" = "Wave 2", the data collected at & \\
Time 2. &
\end{tabular}

Responses from all questions and all time points were grouped together based on the similarity of their content and their applicability to the overall concepts of interest. Codes were developed through a combination of deductive and inductive coding such 
that while some quotes were coded under theoretical constructs (such as, "examples of work-nonwork conflict"), other codes were allowed to emerge organically from the data.

Once an overarching code list was developed and applied, a second party was asked to review the quotes and come up with an independent code list. The two code lists were then compared. Where discrepancies occurred, theory and previous experience guided discussion until an agreement was made. Thus, a single code list was created and applied to the data (see Appendix $\mathrm{H}$ for the code list). The results of this coding are described in the Results section below. 


\section{Chapter 5}

\section{Results}

\section{Demographic Characteristics}

The age of participants in the sample was broadly distributed, ranging from 22 to 75 , with a mean of 40.31 and a standard deviation of 12 . The sample was highly educated; $42.9 \%$ had obtained a graduate degree and $40.6 \%$ had obtained a four year college degree. The sample was heavily female (70.1\%). The majority of the sample lived with their spouse or partner $(75.3 \%)$ rather than as the only adult in the household $(13.5 \%)$ or as an adult living with other adult roommates or adult family members (11.2\%). Respondents reported a wide range of household income, the majority $(58.7 \%)$ falling between $\$ 50,000$ and $\$ 150,000$ before taxes. The median income level was the $\$ 75,000$ to $\$ 99,000$ income category. Industries represented included education services (37.3\%), professional, scientific, and technical services (17.0\%), and health care and social assistance (10.3\%), among others. The majority of respondents (58.9\%) did not have children living in their home. The majority of those with children typically had one or two children living in their home. See Table 1 (correlation table) for descriptive statistics on the continuous variables included in analyses, and Tables 4, 5, 6, 7, 8, and 9 for descriptive statistics on categorical variables. 
Table 4. Descriptive Statistics Regarding Income

\begin{tabular}{lccc}
\hline Income & Frequency & Percent & Valid Percent \\
\hline Less than $\$ 10,000$ & 3.0 & 1.0 & 1.0 \\
Between $\$ 10,000$ and $\$ 14,999$ & 6.0 & 1.9 & 1.9 \\
Between $\$ 15,000$ and $\$ 24,999$ & 14.0 & 4.4 & 4.5 \\
Between $\$ 25,000$ and $\$ 34,999$ & 19.0 & 6.0 & 6.1 \\
Between $\$ 35,000$ and $\$ 49,999$ & 45.0 & 14.3 & 14.6 \\
Between $\$ 50,000$ and $\$ 74,999$ & 55.0 & 17.5 & 17.8 \\
Between $\$ 75,000$ and $\$ 99,000$ & 51.0 & 16.2 & 16.5 \\
Between $\$ 100,000$ and $\$ 149,999$ & 77.0 & 24.4 & 24.9 \\
Between $\$ 150,000$ and $\$ 199,999$ & 22.0 & 7.0 & 7.1 \\
$\$ 200,000$ or more & 17.0 & 5.4 & 5.5 \\
Total & 309.0 & 98.1 & 100.0 \\
Missing & 6.0 & 1.9 & \\
Total & 315.0 & 100.0 & \\
\hline
\end{tabular}

Table 5. Descriptive Statistics Regarding Gender

\begin{tabular}{lrrr}
\hline Gender & Frequency & Percent & Valid Percent \\
\hline Female & 220.0 & 69.8 & 70.1 \\
Male & 94.0 & 29.8 & 29.9 \\
Total & 314.0 & 99.7 & 100.0 \\
Missing & 1.0 & 0.3 & \\
Total & 315.0 & 100.0 & \\
\hline
\end{tabular}


Table 6. Descriptive Statistics Regarding Education

\begin{tabular}{lccc}
\hline Education Level & Frequency & Percent & Valid Percent \\
\hline Grade school or less & 2.0 & 0.6 & 0.6 \\
Some high school & 2.0 & 0.6 & 0.6 \\
High school diploma or equivalency & 4.0 & 1.3 & 1.3 \\
Some college or other higher education, no diploma & 24.0 & 7.6 & 7.6 \\
Trade school completed & 7.0 & 2.2 & 2.2 \\
2 year college degree & 13.0 & 4.1 & 4.1 \\
4 year college degree & 128.0 & 40.6 & 40.6 \\
Graduate degree & 135.0 & 42.9 & 42.9 \\
Total & 315.0 & 100.0 & 100.0 \\
\hline
\end{tabular}

Table 7. Descriptive Statistics Regarding Industry

\begin{tabular}{lccc}
\hline Industry & Frequency & Percent & Valid Percent \\
\hline Agriculture, forestry, fishing and hunting & 9.0 & 2.9 & 2.9 \\
Utilities & 3.0 & 1.0 & 1.0 \\
Construction & 4.0 & 1.3 & 1.3 \\
Manufacturing & 6.0 & 1.9 & 1.9 \\
Wholesale Trade & 3.0 & 1.0 & 1.0 \\
Retail Trade & 5.0 & 1.6 & 1.6 \\
Transportation and Warehousing & 3.0 & 1.0 & 1.0 \\
Information, Finance, and Insurance & 8.0 & 2.5 & 2.6 \\
Real Estate, Rental, and Leasing & 2.0 & 0.6 & 0.6 \\
Professional, Scientific, and Technical Services & 53.0 & 16.8 & 17.0 \\
Management of Companies and Enterprises & 3.0 & 1.0 & 1.0 \\
Administrative and Support & 15.0 & 4.8 & 4.8 \\
Education Services & 116.0 & 36.8 & 37.3
\end{tabular}




\begin{tabular}{lrrr} 
Health Care and Social Assistance & 32.0 & 10.2 & 10.3 \\
Arts, Entertainment, and Recreation & 6.0 & 1.9 & 1.9 \\
Accommodation and Foodservices & 2.0 & 0.6 & 0.6 \\
Other Services & 38.0 & 12.1 & 12.2 \\
Public Administration & 3.0 & 1.0 & 1.0 \\
Total & 311.0 & 98.7 & 100.0 \\
Missing & 4.0 & 1.3 & \\
Total & 315.0 & 100.0 & \\
\hline
\end{tabular}

Table 8. Descriptive Statistics Regarding Household Status at Time 1

\begin{tabular}{lrrc}
\hline Household Status & Frequency & Percent & Valid Percent \\
\hline Living with a spouse or partner & 234.0 & 74.3 & 74.8 \\
Have spouse or partner, not living together & 13.0 & 4.1 & 4.2 \\
Not living with a spouse or partner & 66.0 & 21.0 & 21.1 \\
Total & 313.0 & 99.4 & 100.0 \\
Missing & 2.0 & 0.6 & \\
Total & 315.0 & 100.0 & \\
\hline
\end{tabular}

Table 9. Descriptive Statistics Regarding Household Status at Time 2

\begin{tabular}{lrrc}
\hline Household Status & Frequency & Percent & Valid Percent \\
\hline Living with a spouse or partner & 235.0 & 74.6 & 75.3 \\
Only adult in the household (alone or with children) & 42.0 & 13.3 & 13.5 \\
Living with adult roommates or other adult family members & 35.0 & 11.1 & 11.2 \\
Total & 312.0 & 99.0 & 100.0 \\
Missing System & 3.0 & 1.0 & \\
Total & 315.0 & 100.0 & \\
\hline
\end{tabular}


Analysis of the IP addresses indicated that participants came from at least 24 different states in the United States, including Alaska, Alabama, Arizona, California, Colorado, Florida, Hawaii, Illinois, Indiana, Massachusetts, Maryland, Maine, Minnesota, North Carolina, Nevada, New York, Ohio, Oregon, Pennsylvania, Texas, Utah, Virginia, and Washington. Other participants were from Washington, D.C. and Canada. The majority of the respondents were located in Oregon, California, Washington, Hawaii, and Ohio, respectively.

In the first survey, respondents were asked where they heard about the survey, in order to better track the snowball sampling. See Table 10 for a summary of the survey sources listed by participants. Overall, the majority received the email from myself or someone else $(77.4 \%)$.

Table 10. Survey Source

\begin{tabular}{lrr}
\hline Source & $N$ & $\%$ \\
\hline Email from Jenna LeComte-Hinely & $981.10 \%$ \\
Email from someone other than Jenna LeComte-Hinely & 146 & $46.30 \%$ \\
Internal company email & 13 & $4.10 \%$ \\
Facebook Post from Jenna LeComte-Hinely & 9 & $2.90 \%$ \\
Facebook Post from someone other than Jenna LeComte-Hinely & 38 & $12.10 \%$ \\
PIOPA email list & 5 & $1.60 \%$ \\
Google + Post from Jenna LeComte-Hinely & 1 & $0.30 \%$ \\
Other & 5 & $1.60 \%$ \\
\hline Total & 315 & $100.00 \%$ \\
\hline
\end{tabular}


As is evident from the means and standard deviations presented in Table 1, the majority of individual boundary preferences ranged from neutral to segmented, while the majority of reported boundary supplies ranged from neutral to integrated. It is worth noting that there appears to be issues of range restriction on all six enhancement variables, such that the majority of the sample experienced high levels of enhancement (for example, the average level of nonwork-to-work value-based enhancement was 4.48 on a 5.00 scale). In contrast, there appears to be a downward range restriction on nonwork-to-work conflict, such that the majority of individuals experienced very little conflict in that direction (e.g., the lowest nonwork-to-work conflict score was 3.67 on a 5.00 scale). Thus, results regarding enhancement and nonwork-to-work conflict should be examined with these range restrictions in mind. Other important constructs (i.e., boundary preferences, boundary supplies, work-to-nonwork conflict, mental health, and physical health) did not suffer from severe range restrictions, and thus allow examination of overall trends.

\section{Non-response Analysis}

Independent samples t-tests and chi-square analyses were conducted to assess the potential presence of significant differences between those participants who completed both surveys and those who only completed the first survey. Results showed that there were no significant differences between the two groups at the $p<.05$ level for the majority of the variables, including boundary preferences, boundary supplies, work-tononwork conflict, nonwork-to-work conflict, work-to-nonwork behavior-based enhancement, work-to-nonwork-value based enhancement, work-to-nonwork affective 
enhancement, nonwork-to-work behavior-based enhancement, nonwork-to-work valuebased enhancement, nonwork-to-work affective enhancement, gender, age, and number of children in the household.

The only significant difference between the two groups was in regards to the variable of education. Individuals who completed only the first survey had significantly lower levels of education $(M=6.73)$ than those who completed the survey at both time points $(M=6.96), t(588.276)=-2.005, p<.05$ (equal variances not assumed, as per a significant Levine's test). Given that the education variable was on an 8-point scale (ranging from "less than high school" to "graduate degree"), this difference does not appear to be practically meaningful.

There was also no significant difference between individuals who completed the survey at both time points and those who only completed the Time 1 survey on the physical health composite score, $t(528)=.980, p>.05$. Similarly, there was no significant difference between the two groups on the mental health composite score, $t$ $(528)=.965, p>.05$. One caveat is worth noting, however. When conducting the analyses for this dissertation as a whole, I obtained a limited number of scoring licenses from QualityMetric, the owner of the SF-12 scale. Due to the relatively small number of licenses, I did not score all survey responses, but rather, only those who had completed the survey at both time points. In order to conduct these post-hoc non-responder analyses, I scored the PCS and MCS scores for those individuals who had only completed the survey at Time 1 for the first time. By this time, I did not have sufficient scoring licenses to score all of the individuals who only completed the survey at Time 1, and was 
only able to score 215 of the 293 possible responses. Thus, the post-hoc non-responder analyses pertaining to the composite scores compared 215 individuals who had responded at Time 1 only with the 315 individuals who responded at both time points. All other post-hoc non-responder analyses include all 293 individuals who completed only the Time 1 survey, as there was no need to obtain scoring licenses for the other variables.

Overall, the results of these post-hoc independent t-tests and chi-square analyses indicate that there are no crucial significant differences on the major variables of interest between individuals who responded at both time points and those who did not respond to the Time 2 survey. These findings do not rule out the possibility that non-responders to the second survey differed significantly from those who completed both surveys. It is entirely possible that the group that did not respond to the second survey experienced different events during the time lag, which could have resulted in significantly different Time 2 responses. However, the lack of significant differences in Time 1 scores do imbue the findings with greater confidence regarding potential response bias; it appears unlikely that any significant changes were the result of initial group differences rather than true changes.

\section{Primary Analysis}

Primary analyses were conducted via LCM to assess the path coefficients pertaining to each of the hypotheses. See Table 2 for the list of the 24 models used to test these hypotheses.

Hypothesis 1. Hypothesis 1a stated that work-to-nonwork interference would have a significant negative effect on health. Twelve models tested this hypothesis, six for 
mental health and six for physical health. All six models that used the outcome of mental health were significant. This includes Model $4(\beta=-.318, B=-5.734, p<.001)$, Model 5 $(\beta=-.316, B=-5.690, p<.001)$, Model $12(\beta=-.320, B=-5.783, p<.001)$, Model $13(\beta$ $=-.318, B=-5.737, p<.001)$, Model $20(\beta=-.319, B=-5.749, p<.001)$, and Model 21 $(\beta=-.327, B=-5.891, p<.001)$. However, none of the six models that used the outcome of physical health were significant. This includes Model 3 ( $\beta=-.121, B=-$ $1.641, p>.05)$, Model $6(\beta=-.141, \mathrm{~B}=-1.905, p>.05)$, Model $11(\beta=-.116, \mathrm{~B}=-$ $1.564, p>.05$, Model $14(\beta=-.115, B=-1.552, p>.05)$, Model $19(\beta=-.118, B=-$ $1.589, p>.05)$, and Model $22(\beta=-.125, B=-1.680, p>.05)$. Thus, it appears that Hypothesis 1a was partially supported; work-to-nonwork conflict was consistently related to decreased mental health, but not to decreased physical health.

Hypothesis $1 \mathrm{~b}$ stated that nonwork-to-work conflict would have a significant negative effect on health. Twelve models tested this hypothesis, six for mental health and six for physical health. All six models that used the outcome of mental health were significant. This includes Model $1(\beta=-.334, B=-5.602, p<.001)$, Model $8(\beta=-.340$, $B=5.705, p<.001)$, Model $9(\beta=-.332, B=-5.571, p<.001)$, Model $16(\beta=-.340, B=$ $-5.718, p<.001)$, Model $17(\beta=-.335, B=-5.618, p<.001)$, and Model $24(\beta=-.339, B$ $=-5.721, p<.001)$. However, none of the six models that used the outcome of physical health were significant. This includes Model $2(\beta=-.027, B=-.334, p>.05)$, Model 7 ( $\beta$ $=-.015, B=-.184, p>.05)$, Model $10(\beta=-.030, B=-.377, p>.05)$, Model $15(\beta=-$ $.017, B=-.212, p>.05)$, Model $18(\beta=-.031, B=-.390, p>.05)$, and Model $23(\beta=-$ $.021, B=-.268, p>.05)$. Thus, it appears that Hypothesis $1 \mathrm{~b}$ was partially supported; 
nonwork-to-work conflict was consistently related to decreased mental health, but not to decreased physical health.

Hypothesis 2. Hypothesis 2a stated that work-to-nonwork behavior-based enhancement would be positively related to health. Four models tested this hypothesis, two for mental health (Model 1, $\beta=-.038, B=-.509, p>.05$, and Model 4, $\beta=-.021, B=$ $-.288, p>.05$ ) and two for physical health (Model 2, $\beta=.082, B=.826, p>.05$, and Model 3, $\beta=.086, B=.873, p>.05)$. None of these models showed a significant path between the two variables, and therefore, Hypothesis 2 a was not supported.

Hypothesis $2 \mathrm{~b}$ stated that work-to-nonwork value-based enhancement would be positively related to health. Four models tested this hypothesis, two for mental health (Model 9, $\beta=-.051, B=-.589, p>.05$, and Model 12, $\beta=-.065, B=-.752, p>.05$ ) and two for physical health (Model 10, $\beta=.066, B=.569, p>.05$, and Model 11, $\beta=.063, B$ $=.544, p>.05)$. None of these models showed a significant path between the two variables, and therefore, Hypothesis $2 \mathrm{~b}$ was not supported.

Hypothesis 2c stated that work-to-nonwork affective enhancement would be positively related to health. Four models tested this hypothesis, two for mental health (Model 5 and Model 8) and two for physical health (Model 6 and Model 7). The two models that used physical health as an outcome, Model $6(\beta=.167, B=2.688, p<.01)$ and Model 7 ( $\beta=.152, B=2.452, p<.01)$, had significant and positive pathways between the two variables. However, the two models that used mental health as the outcome, Model $5(\beta=-.021, B=-.448, p>.05)$ and Model $8(\beta=-.080, B=-1.734, p>$ .05) did not have any significant pathways. Thus, Hypothesis 2c was partially supported; 
it appears that work-to-nonwork affective enhancement is significantly and positively related to physical health, but not to mental health.

Hypothesis 2d stated that nonwork-to-work behavior-based enhancement would be positively related to health. Four models tested this hypothesis, two for mental health (Model 13, $\beta=.019, B=.336, p>.05$, and Model 16, $\beta=-.036, B=-.647, p>.05)$ and two for physical health (Model 14, $\beta=.062, B=.836, p>.05$, and Model 15, $\beta=.062, B$ $=.838, p>.05)$. None of these models showed a significant path between the two variables, and therefore, Hypothesis $2 \mathrm{~d}$ was not supported.

Hypothesis 2e stated that nonwork-to-work value-based enhancement would be positively related to health. Four models tested this hypothesis, two for mental health (Model 21, $\beta=.064, B=1.204, p>.05$, and Model 24, $\beta=-.034, B=-.634, p>.05$ ) and two for physical health (Model 22, $\beta=.056, B=.780, p>.05$, and Model 23, $\beta=.039, B$ $=.550, p>.05)$. None of these models showed a significant path between the two variables, and therefore, Hypothesis 2e was not supported.

Hypothesis $2 \mathrm{f}$ stated that nonwork-to-work affective enhancement would be positively related to health. Four models tested this hypothesis, two for mental health (Model 17 and Model 20) and two for physical health (Model 18 and Model 19). The two models that used physical health as an outcome, Model $18(\beta=.114, B=1.294, p<$ $.05)$ and Model $19(\beta=.113, B=1.287, p<.05)$, had significant and positive pathways between the two variables. However, the two models that used mental health as the outcome, Model $17(\beta=.038, B=.573, p>.05)$ and Model $20(\beta=.030, B=.452, p>$ .05), did not have any significant pathways. Thus, Hypothesis $2 \mathrm{f}$ was partially supported; 
it appears that nonwork-to-work affective enhancement is significantly and positively related to physical health, but not to mental health.

Hypothesis 3. Hypothesis 3a stated that good fit, or congruence, between individual work-nonwork boundary management preferences and organizational worknonwork boundary management supplies would be positively related to mental health. Twelve models tested this hypothesis. Results showed that in all 12 of the models, the pathway between congruence and mental health was significant and positive. Note that the actual coefficients are negative, as LCM conceptualizes "congruence" as the amount of difference between preferences and supplies, where a large gap results in a high number, indicating less congruence. For the ease of interpretation, the signs of all path coefficients listed here have been reversed, such that a positive path coefficient indicates that a greater amount of congruence is associated with a higher amount of mental health. This sign reversal is standard practice for examining fit via LCM (Chen et al., 2009). This included Model $1(\beta=.319, B=1.389, p<.001)$, Model $4(\beta=.179, B=.779, p<$ $.01)$, Model $5(\beta=.177, B=.768, p<.01)$, Model $8(\beta=.315, B=1.370, p<.001)$, Model $9(\beta=.322, B=1.398, p<.001)$, Model $12(\beta=.187, B=.812, p<.01)$, Model $13(\beta=.175, B=.760, p<.01)$, Model $16(\beta=.309, B=1.346, p<.001)$, Model $17(\beta=$ $.307, B=1.335, p<.001)$, Model $20(\beta=.171, B=.743, p<.01)$, Model $21(\beta=.170, B$ $=.741, p<.01)$, and Model $24(\beta=.310, B=1.347, p<.001)$. Thus, Hypothesis $3 \mathrm{a}$ is fully supported.

Hypothesis $3 b$ stated that good fit, or congruence, between individual worknonwork boundary management preferences and organizational work-nonwork boundary 
management supplies would be positively related to physical health. Twelve models tested this hypothesis. Results showed that in ten of the 12 models, the pathway between congruence and physical health was significant, but negative, in the opposite direction of the hypothesis (note that once again, the signs of these path coefficients have been reversed to ease interpretation, thus, a negative path coefficient indicates that a greater amount of congruence is associated with a smaller amount of physical health). This included Model $2(\beta=-.126, B=-.411, p<.05)$, Model $3(\beta=-.184, B=-.600, p<.01)$, Model $6(\beta=-.183, B=-.594, p<.01)$, Model $7(\beta=-.115, B=-.373, p<.05)$, Model 10 $(\beta=-.121, B=-.395, p<.05)$, Model $11(\beta=-.175, B=-.568, p<.05)$, Model $14(\beta=-$ $.159, B=-.517, p<.05)$, Model $18(\beta=-.119, B=-.388, p<.05)$, Model $19(\beta=-.174, B$ $=-.566, p<.05)$, Model $22(\beta=-.164, B=-.535, p<.05)$. In two of the models, Model $15(\beta=-.105, B=-.342, p>.05)$ and Model $23(\beta=-.106, B=-.344, p>.05)$, congruence did not have a significant relationship with physical health. Thus, Hypothesis $3 b$ is not supported.

Hypothesis 4. Hypothesis 4a stated that there would be a significant positive indirect effect of work-nonwork boundary fit on health through work-to-nonwork conflict. Twelve models tested this hypothesis, six with the outcome of mental health (Model 4, Model 5, Model 12, Model 13, Model 20, and Model 21) and six with the outcome of physical health (Model 3, Model 6, Model 11, Model 14, Model 19, Model 22). The Sobel test indicated that after reversing the signs for the congruence coefficients, there was a significant positive indirect effect of congruence on mental health through work-to-nonwork for the six models, including Model $5(Z=4.667, p<$ 
$.001)$, Model $4(Z=3.446, p<.001)$, Model $12(Z=4.670, p<.001)$, Model $13(Z=$ 4.676, $p<.001)$, Model $20(Z=4.664, p<.001)$, Model $21(Z=4.734, p<.001)$.

However, none of the six models that tested the indirect pathway from congruence to physical health via work-to-nonwork conflict were significant at the $p<$ .05 level. This includes Model $3(Z=1.639, p>.05)$, Model $6(Z=1.882, p>.05)$, Model $11(Z=1.568, p>.05)$, Model $14(Z=1.555, p>.05)$, Model $19(Z=1.598, p>$ $.05)$, and Model $22(Z=1.664, p>.05)$. Thus, Hypothesis $4 \mathrm{a}$ is partially supported; it appears that there is a significant positive indirect effect of congruence on mental health through work-to-nonwork conflict, but that the same relationship does not hold true for physical health.

Hypothesis $4 \mathrm{~b}$ stated that there would be a significant positive indirect effect of work-nonwork boundary fit on health through nonwork-to-work conflict. Twelve models tested this hypothesis, six with the outcome of mental health (Model 1, Model 8, Model 9, Model 16, Model 17, Model 24) and six with the outcome of physical health (Model 2, Model 7, Model 10, Model 15, Model 18, Model 23). Sobel tests were used to assess the significance of the indirect effects. None of the six models pertaining to mental health resulted in significant indirect effects at the $p<.05$ level as hypothesized (all six models $Z=.805, p>.05)$. Similarly, none of the six models pertaining to physical health results in significant indirect effects at the $\mathrm{p}<.05$ level as hypothesized, including Model 2 ( $Z=$ $.404, p>.05)$, Model $7(Z=.239, p>.05)$, Model $10(Z=.432, p>.05)$, Model $15(Z=$ $.265, p>.05)$, Model $18(Z=.445, p>.05)$, and Model $23(Z=.324, p>.05)$. Thus, Hypothesis $4 \mathrm{~b}$ was not supported. 
Hypothesis 5. Hypothesis 5a stated that there would be a significant positive indirect effect of work-nonwork boundary fit on health through work-to-nonwork behavior-based enhancement. Four models tested this hypothesis using the Sobel test, two with the outcome of mental health (Model 1, Z =-.684, $p>.05$, and Model 4, $Z=-$ $.384, p>.05$ ) and two with the outcome of physical health (Model $2, Z=1.263, p>.05$ and Model 3, $Z=1.329, p>.05)$. None of the four models resulted in significant indirect paths as hypothesized. Thus, Hypothesis 5a was not supported.

Hypothesis $5 \mathrm{~b}$ stated that there would be a significant positive indirect effect of work-nonwork boundary fit on health through work-to-nonwork value-based enhancement. Four models tested this hypothesis using the Sobel test, two with the outcome of mental health (Model 9, $Z=-.913, p>.05$, and Model 12, $Z=-1.123, p>$ .05 ) and two with the outcome of physical health (Model 10, $Z=1.039, p>.05$, and Model 11, $Z=1.000, p>.05)$. None of the four models resulted in significant indirect paths as hypothesized. Thus, Hypothesis $5 \mathrm{~b}$ was not supported.

Hypothesis $5 \mathrm{c}$ stated that there would be a significant positive indirect effect of work-nonwork boundary fit on health through work-to-nonwork affective enhancement. Four models tested this hypothesis using the Sobel test, two with the outcome of mental health (Model 5, $Z=-.358, p>.05$, and Model 8, $Z=-.738, p>.05$ ) and two with the outcome of physical health (Model 6, $Z=.811, p>.05$, and Model 7, $Z=.804, p>.05$ ). None of the four models resulted in significant indirect paths as hypothesized. Thus, Hypothesis $5 \mathrm{c}$ was not supported. 
Hypothesis $5 \mathrm{~d}$ stated that there would be a significant positive indirect effect of work-nonwork boundary fit on health through nonwork-to-work behavior-based enhancement. Four models tested this hypothesis using the Sobel test, two with the outcome of mental health (Model 13, $Z=-.279, p>.05$, and Model 16, $Z=.383, p>.05$ ) and two with the outcome of physical health (Model 14, $Z=-.374, p>.05$, and Model $15, Z=-.373, p>.05)$. None of the four models resulted in significant indirect paths as hypothesized. Thus, Hypothesis $5 \mathrm{~d}$ was not supported.

Hypothesis 5e stated that there would be a significant positive indirect effect of work-nonwork boundary fit on health through nonwork-to-work value-based enhancement. Four models tested this hypothesis with the Sobel test, two with the outcome of mental health (Model 21, $Z=-.211, p>.05$, and Model 24, $Z=.202, p>.05$ ) and two with the outcome of physical health (Model 22, $Z=-.209, p>.05$, and Model $23, Z=-.203, p>.05)$. None of the four models resulted in significant indirect paths as hypothesized. Thus, Hypothesis 5e was not supported.

Hypothesis $5 \mathrm{f}$ stated that there would be a significant positive indirect effect of work-nonwork boundary fit on health through nonwork-to-work affective enhancement. Four models tested this hypothesis using the Sobel test, two with the outcome of mental health (Model 17, $Z=.677, p>.05$, and Model 20, $Z=.542, p>.05$ ) and two with the outcome of physical health (Model 18, $Z=1.278, p>.05$, and Model 19, $Z=1.040, p>$ $.05)$. None of the four models resulted in significant indirect paths as hypothesized. Thus, Hypothesis $5 \mathrm{f}$ was not supported.

For clarity, these results are summarized in Table 11. 
Table 11. Summary of Hypotheses and Results

\begin{tabular}{|c|c|c|c|}
\hline \multicolumn{2}{|c|}{ Hypothesis } & \multirow[t]{2}{*}{ Support } & \multirow[t]{2}{*}{ Notes } \\
\hline 1 & & & \\
\hline $1 \mathrm{a}$ & Work-to-nonwork conflict $\rightarrow$ health & Partial & Mental only \\
\hline $1 b$ & Nonwork-to-work conflict $\rightarrow$ health & Partial & Mental only \\
\hline \multicolumn{4}{|l|}{2} \\
\hline $2 \mathrm{a}$ & Work-to-nonwork behavior enhance $\rightarrow$ health & None & \\
\hline $2 b$ & Work-to-nonwork value enhance $\rightarrow$ health & None & \\
\hline $2 \mathrm{c}$ & Work-to-nonwork affect enhance $\rightarrow$ health & Partial & Physical only \\
\hline $2 \mathrm{~d}$ & Nonwork-to-work behavior enhance $\rightarrow$ health & None & \\
\hline $2 \mathrm{e}$ & Nonwork-to-work value enhance $\rightarrow$ health & None & \\
\hline $2 \mathrm{f}$ & Nonwork-to-work affect enhance $\rightarrow$ health & Partial & Physical only \\
\hline \multicolumn{4}{|l|}{3} \\
\hline $3 \mathrm{a}$ & Congruence $\rightarrow$ mental health & Full & 12 of 12 \\
\hline $3 b$ & Congruence $\rightarrow$ physical health & None & Opposite \\
\hline \multicolumn{4}{|l|}{4} \\
\hline $4 a$ & Congruence $\rightarrow$ work-to-nonwork conflict $\rightarrow$ health & Partial & Mental only \\
\hline $4 b$ & Congruence $\rightarrow$ nonwork-to-work conflict $\rightarrow$ health & None & \\
\hline \multicolumn{4}{|l|}{5} \\
\hline $5 \mathrm{a}$ & Congruence $\rightarrow$ work-to-nonwork behavior enhance $\rightarrow$ health & None & \\
\hline $5 b$ & Congruence $\rightarrow$ work-to-nonwork value enhance $\rightarrow$ health & None & \\
\hline $5 c$ & Congruence $\rightarrow$ work-to-nonwork affect enhance $\rightarrow$ health & None & \\
\hline $5 \mathrm{~d}$ & Congruence $\rightarrow$ nonwork-to-work behavior enhance $\rightarrow$ health & None & \\
\hline $5 e$ & Congruence $\rightarrow$ nonwork-to-work value enhance $\rightarrow$ health & None & \\
\hline $5 f$ & Congruence $\rightarrow$ nonwork-to-work affect enhance $\rightarrow$ health & None & \\
\hline
\end{tabular}


Additional Considerations in Hypothesis Testing. While the 24 LCMs

described above do test the proposed hypotheses, several questions remained

unanswered. Thus, three additional sets of analyses were conducted: assessing the LCMs with Time 2 mediator data, assessing the LCMs with the additional control variable of Time 1 health, and assessing the LCM models without the mediators.

Models with Time 2 Mediators. The ideal way in which to measure these hypotheses would be with a design with three time points, i.e., boundary preferences and boundary supplies from a Time 1 data collection, conflict and enhancement from Time 2 , and health from Time 3 (Shrout \& Bolger, 2002). This method would allow for greater causal inference. However, this study did not contain three time points, and thus the decision of whether to use Time 1 data or Time 2 data for the mediators must be made. This is a difficult decision, as there is no clear theoretical guidance on which to choose, as mentioned previously. As discussed in the methods section, the stressor-strain model of stress implies that in order to experience health outcomes as a result of a stressor, an individual must experience the stressor, appraise the stressor as threatening, and attempt to cope with the stressor (Darr \& Johns, 2008; Edwards, 1992; Hart \& Cooper, 2001; Hurrell et al., 1998; Lazarus, 1990). The depletion of coping resources then triggers psychological and/or physical symptomology (Hockey, 1993; Melamed et al., 2006). This theory does not explicitly address how long it takes a stressor to develop into health outcomes. However, the multiple steps between stressor and strain, as well as the connotations of the depletion process, imply that this is a process that occurs over time. Thus, it would be most logical to model the data with the stressors all from Time 1 in 
order to allow them sufficient time to have an impact on health. However, given the lack of theoretical guidance, I chose to support my logic with additional evidence. Thus, I also ran the same 24 models with the mediator variables gathered from Time 2 data collection. See Appendix J for a list of the significant findings that occurred when using Time 2 mediator data instead of Time 1 mediator data.

The results of this second set of analyses showed that many of the trends that appeared in the first round of analyses were sustained. For example, work-to-nonwork conflict again had a significant negative effect on mental health in all six of the models that included those two variables (partially supporting Hypothesis 1a). Similarly, nonwork-to-work conflict again had a significant negative relationship with mental health in all six of the models that included those two variables (partially supporting Hypothesis 1b). Congruence was again related significantly and negatively to physical health (11 out of the 12 models; Hypothesis 3a) and significantly and positively to mental health (12 out of the 12 models; Hypothesis $3 b$ ). In terms of indirect effects, once again, congruence had a significant negative indirect effect on mental health via work-to-nonwork conflict (partially supporting Hypothesis 4a).

However, there were some additional results that were significant when using Time 2 mediators that were not significant when using mediator data from Time 1. For instance, work-to-nonwork conflict now had a significant negative relationship with physical health as well as the mental health relationship demonstrated with Time 1 mediators (in five out of the six models that measured the relationship; Hypothesis 1a). This also meant that the indirect effects of congruence on physical health via work-to- 
nonwork conflict became statistically significant (Hypothesis 4a), providing additional support for this hypothesis. These two findings expand the Time 1 mediator findings by broadening the influence of work-to-nonwork conflict to physical health in addition to mental health.

Other significant effects also appeared that were not in a consistent pattern with the results from the analyses using Time 1 mediator data. For example, in one out of four models pertaining to Hypothesis 2a, work-to-nonwork behavior-based enhancement was now significantly and positively linked to physical health. Work-to-nonwork value-based behavior was significantly and positively linked to mental health in one of four models pertaining to Hypothesis $2 \mathrm{~b}$. There were several other instances where using Time 2 mediator data instead of Time 1 mediator data changed a path to significant in one of the several models used to test that hypothesis. It may be that these "one-off" relationships became significant simply due to measurement proximity, rather than actual construct difference.

I decided to base my conclusions off of the analyses conducted using Time 1 mediator data for several reasons. First, I wanted to be conservative and not claim findings as "true" if they were not, in fact, accurate. In this sense, since the analyses using Time 1 mediators had fewer significant findings, it was logical to use these analyses. Additionally, all of the major patterns found in Time 1 models were also found when running analyses on models with Time 2 mediator data-if anything changed, the support for the hypotheses were strengthened. Thus, it appears that the patterns established in the Time 1 models are accurate. Next, the Time 1 models had a 
consistency that the additional findings from Time 2 did not—-the patterns were clear and consistent. In much of the additional findings from the Time 2 models, only one out of many models that included the relationship became significant, which does not lend great confidence that this relationship is a product of anything other than measurement differences. Thus, in order to have the maximum amount of confidence in the results, I chose to utilize the models that used Time 1 mediator data. Further details regarding the analyses of the Time 2 mediator data models is available upon request.

Models Controlling for Time 1 Health. The finding that congruence was positively related to mental health but negatively related to physical health was unexpected and not supported by previous literature. Divergent findings such as these may be due to several factors, including 1) calculation errors, 2) statistical artifacts, such as suppression or colinearity, 3) unique aspects of the non-random sample, 4) the influence of an un-measured "third variable", and 5) the findings may be representative of a true relationship that has not yet been examined in the literature. Given that both of the 24 LCMs with Time 2 mediators yielded the same pattern of responses as the 24 LCMs with Time 1 mediators, it is unlikely that these findings are due to calculation errors.

In an attempt to assess the third category, unique aspects of the non-random sample, the same 24 models were run a third time. In this series, the predictor and the mediator were both selected from Time 1 data, while the outcome was from Time 2 data. An additional control variable was added to each of these models, however; the appropriate health data from Time 1. The purpose of this was to assess if the proposed 
model accounted for change in health over time, that is, health at Time 2 when controlling for Time 1. Given that the initial results showed that congruence was positive for mental health but negative for physical health, it is possible that this was due simply to having a sample that overall had a poor level of physical health. These 24 models that controlled for Time 1 health were instrumental in assessing if that was in fact the case.

Fit indices for these additional 24 LCMs all maintained acceptable levels of fit (i.e., CFI $>.95$, RMSEA $<.10$; Kline, 2012). Results showed that while many (although not all) of the paths of interest became non-significant when controlling for Time 1 health, the pattern of directionality remained the same. That is, congruence was still beneficial for mental health and detrimental for physical health, although often no longer at a statistically significant level. Thus, it appears that overall physical health level is not a potential explanation for the divergent mental-physical health findings. Additional information regarding these 24 additional LCMs (e.g., output, etc.) is available upon request.

Models without Mediators. Next, to address the possibility that the divergent mental-physical health findings were due to statistical artifacts such as suppression or colinearity, two additional LCMs were conducted without mediators. The rationale behind these analyses was that the 24 models used to test hypotheses essentially tested the hypothesized paths while controlling for many other variables. For example, while the hypothesized path was a positive coefficient from congruence to physical health (Hypothesis 3b), the LCMs that tested that hypothesis tested whether congruence has a positive, significant path to physical health when controlling for conflict, enhancement, 
boundary level, age, number of children, education, and income. If these variables have colinearity problems, it could be masking the true relationships. Thus, in order to assess this, two additional LCMs were conducted without the influence of mediators. See Figure 3 for a visual representation of the simplified model.

Figure 3. Simplified LCM without Mediators

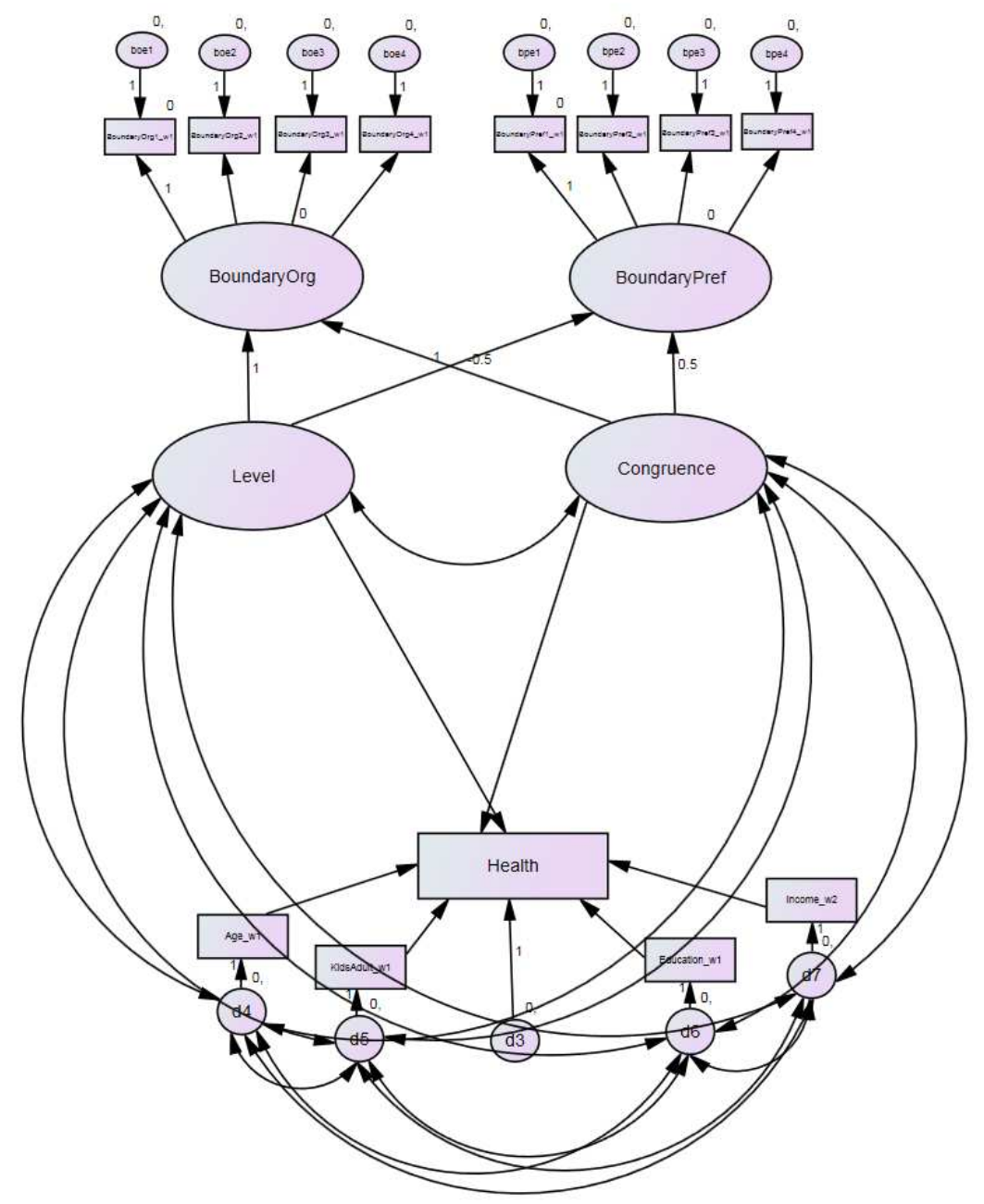

Fit indices indicated that both models had adequate fit as per conventional standards (RMSEA <.10, CFI > .950; Kline, 2012). See Appendix I for the output of 
these two LCMs. Results from the model with physical health as the outcome of interest indicated that there was not a significant path between congruence and physical health, $\beta$ $=-.106, B=-.344, p>.05$. Results from the model with mental health as the outcome of interest indicated that there was a significant relationship between congruence and mental health, $\beta=.327, B=1.424, p<.001$. Note that as in hypothesis testing, the path coefficient signs have been reversed to ease interpretation, such that a positive path coefficient indicates that a greater amount of congruence is associated with a higher amount of mental health. This sign reversal is standard practice for examining fit via LCM (Chen et al., 2009).

Results of these simplified LCMs demonstrated the same general pattern as the 24 LCMs that were conducted with mediator variables. It appears that overall, congruence has a strong positive effect on mental health, and a weaker negative effect on physical health. The fact that these additional, simplified LCMs show the same general pattern as the more complex LCMs used to test the hypotheses indicates that the results of the primary analyses are not likely artifacts of suppression or other statistical issues. Thus, the divergent mental-physical health findings must be explained via theoretical, rather than statistical explanations. This topic will be discussed in depth in the discussion section.

\section{Supplementary Analyses}

Polynomial regression was used to assess the research question regarding potential differential effects of fit directionality on the outcomes of interest. First, a polynomial regression equation was created to map the influence of boundary preference 
and boundary supplies on the outcomes of interest (health, conflict, and enhancement). Response surface mapping of this polynomial regression equation allows for a visual representation of potential imbalance in outcomes.

While these surfaces allow for a visual understanding of the relationship, significance testing of the regression coefficients in the polynomial regression equation allowed for statistical evidence of whether the observed differences were truly statistically significant, or merely negligible. If there was truly no difference in the type of fit (i.e., high preferences-high supplies and low preferences-low supplies are equally good for outcomes) the slope of the line $P=S$ would be zero. Thus, the coefficients along the $P=S$ line were examined to see if there was a significant differences from zero, which would indicate that one type of "good fit" was better for the outcome than the other type of "good fit". Additionally, the $P=-S$ line was examined for differential outcomes in misfit. If there were truly no differences in the direction of misfit (i.e., high preferences-low supplies and low preferences-high supplies are equally detrimental to outcomes), the $P=-S$ line would be a perfect parabola, with the turning point in the center (Edwards \& Rothbard, 1999). Thus, the coefficients of the $P=-S$ line were examined for departures from the expected significant quadratic term. See Table 13 for the significance testing results. 
Table 12. Polynomial Regression Coefficient Significance Testing of Boundary Fit on Health, Conflict, and Enhancement

\begin{tabular}{|c|c|c|c|c|c|c|c|c|c|c|}
\hline & \multicolumn{6}{|c|}{ Results from quadratic regression } & \multicolumn{2}{|c|}{$\begin{array}{l}\text { Shape along the } \\
\qquad P=S \text { line }\end{array}$} & \multicolumn{2}{|c|}{$\begin{array}{l}\text { Shape along the } \\
\qquad P=-S \text { line }\end{array}$} \\
\hline & $\begin{array}{l}P \\
\left(b_{l}\right)\end{array}$ & $\begin{array}{l}S \\
\left(b_{2}\right)\end{array}$ & $\begin{array}{l}P^{2} \\
\left(b_{3}\right)\end{array}$ & $\begin{array}{l}P S \\
\left(b_{4}\right)\end{array}$ & $\begin{array}{l}S^{2} \\
\left(b_{5}\right)\end{array}$ & $R^{2}$ & $b_{1}+b_{2}$ & $\begin{array}{l}b_{3}+b_{4}+ \\
b_{5}\end{array}$ & $b_{1}-b_{2}$ & $\begin{array}{l}b_{3}-b_{4} \\
+b_{5} \\
\end{array}$ \\
\hline \multicolumn{11}{|l|}{ Health } \\
\hline Mental health & $-1.586 * * *$ & $1.487 * * *$ & -.379 & .132 & .045 & $.088 * * *$ & -.100 & -.201 & $-3.073 * * *$ & -.466 \\
\hline Physical Health & .466 & $-.667 * *$ & .286 & .008 & .154 & $.024 *$ & -.200 & $.448 *$ & $1.133 *$ & .432 \\
\hline \multicolumn{11}{|l|}{ Conflict } \\
\hline Work-to-Nonwork Conflict & $.093 *$ & $-.283 * * *$ & .006 & $-.059 * * *$ & $.048 * *$ & $.306 * * *$ & $-.189 * * *$ & -.006 & $.376 * * *$ & $.113 * * *$ \\
\hline Nonwork-to-Work Conflict & .023 & -.013 & .016 & .007 & $-.027 *$ & .002 & .010 & -.003 & .036 & -.017 \\
\hline \multicolumn{11}{|l|}{ Enhancement } \\
\hline Work-to-Nonwork Behavior & & & & & & & & & & \\
\hline $\begin{array}{l}\text { Enhancement } \\
\text { Work-to-Nonwork Value }\end{array}$ & $-.097 *$ & .026 & .029 & -.017 & .008 & $.059 * * *$ & -.071 & .020 & $-.122 *$ & .053 \\
\hline $\begin{array}{l}\text { Enhancement } \\
\text { Work-to-Nonwork }\end{array}$ & $-.131 * *$ & .017 & .005 & .006 & -.001 & $.040 * *$ & $-.114^{*}$ & .009 & $-.148 * *$ & -.002 \\
\hline $\begin{array}{l}\text { Affective Enhancement } \\
\text { Nonwork-to-Work Behavior }\end{array}$ & -.020 & -.021 & -.007 & -.009 & $.026^{*}$ & .000 & -.041 & .010 & .001 & .029 \\
\hline Enhancement & -.003 & $-.044 *$ & .006 & .003 & $.028^{*}$ & .011 & -.046 & .038 & .041 & .031 \\
\hline Nonwork-to-Work Value & & & & & & & & & & \\
\hline Enhancement & -.012 & $-.051 * *$ & .004 & -.013 & $.040 * *$ & $.040 * *$ & -.064 & .031 & .039 & $.057 * *$ \\
\hline Nonwork-to-Work & & & & & & & & & & \\
\hline Affective Enhancement & -.030 & .013 & .013 & .002 & .006 & .001 & -.016 & .022 & -.043 & .017 \\
\hline
\end{tabular}


As is evident from Table 13, four of the ten polynomial regression models are non-significant (nonwork-to-work conflict, work-to-nonwork affective enhancement, nonwork-to-work behavior-based enhancement, and nonwork-to-work affective enhancement). Response surface graphs and additional analyses pertaining to these polynomial regressions are not covered here for the sake of parsimony. These can be found in Appendix K.

Health. The potential differential effects of fit directionality were assessed on two outcomes for health: mental health and physical health.

Mental Health. Figures 4 and 5 illustrate the response surface map for congruence and mental health. From the curvature of the surface in Figure 4, it appears that mental health is slightly higher when boundary supplies are high (i.e., the individual experiences a highly segmented work environment), and that the lowest health outcomes occur when boundary preferences are high but supplies are low (i.e., the individual wants a segmented workplace but works in a highly integrated work environment). 
Figure 4. Response Surface Map of Congruence \& Mental Health

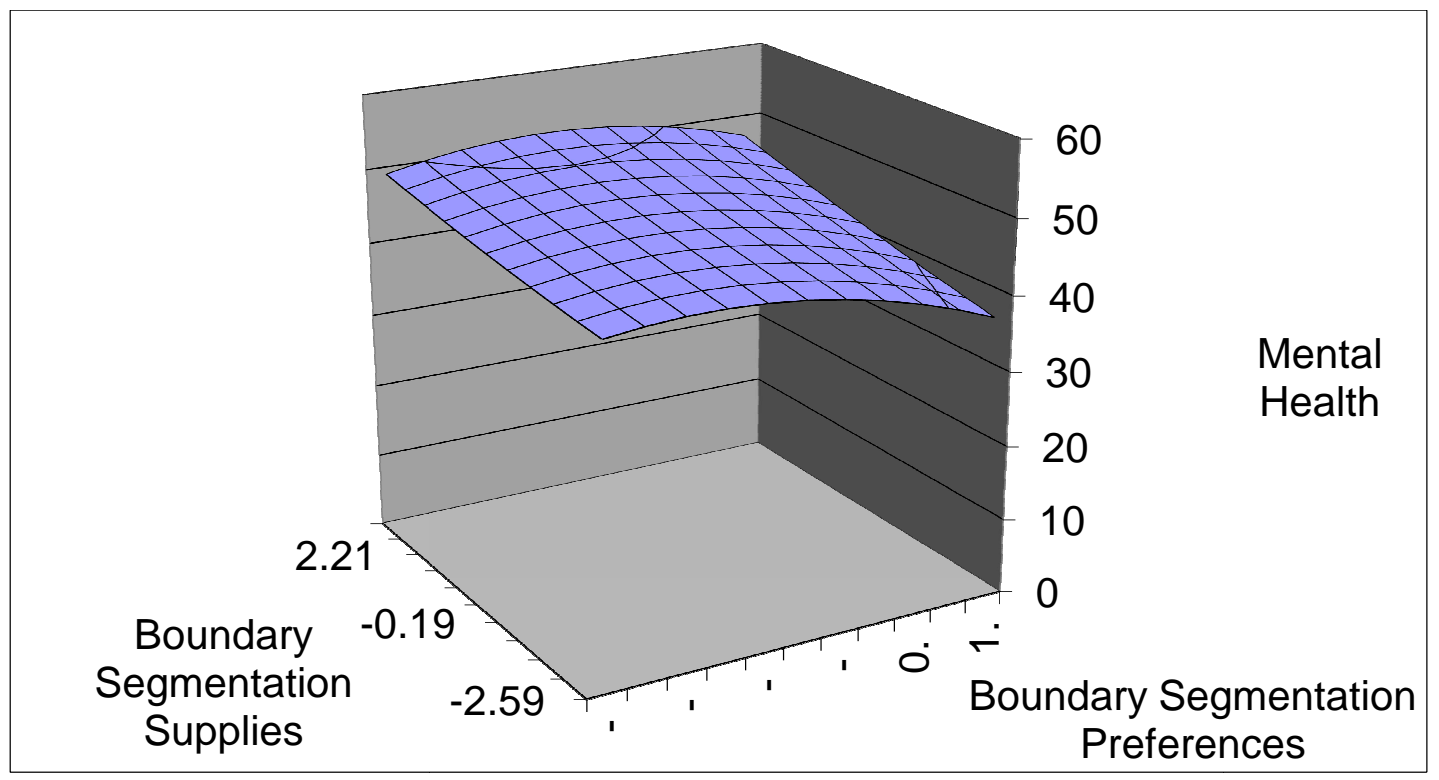

Figure 5. $P=S$ and $P=-S$ Slopes for Congruence \& Mental Health
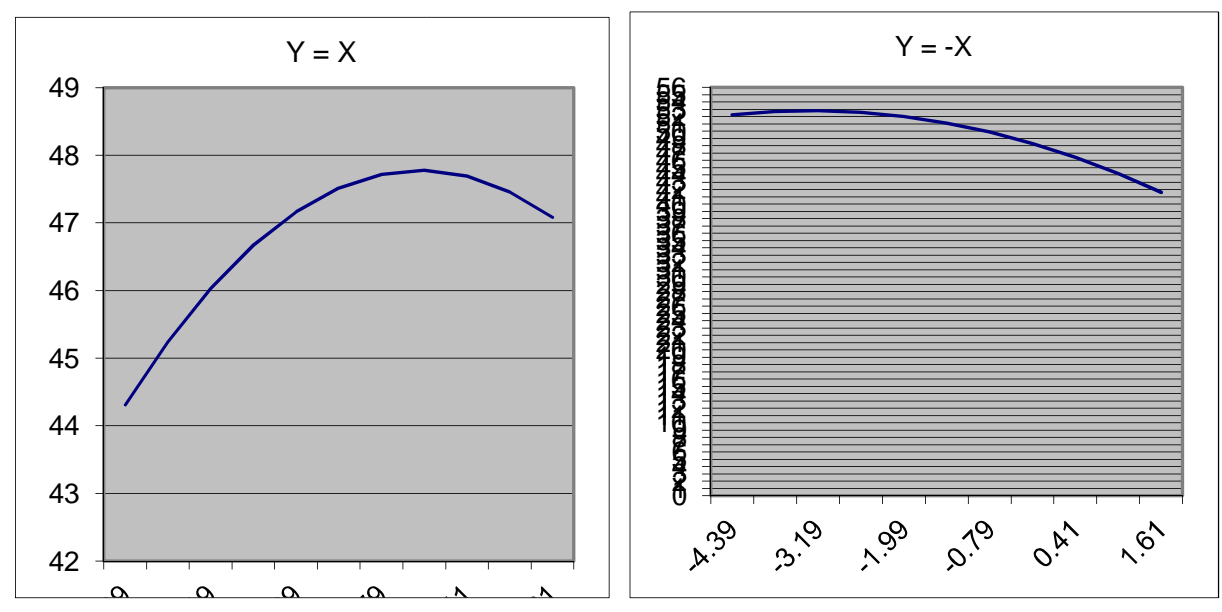

The lack of significant coefficients along the $P=S$ line indicates that instances of perfect fit have the same impact on mental health, regardless of where they fall on the segmentation continuum. Follow-up t-tests were conducted with individuals who experienced two "extremes" of good fit: those who wanted and obtained segmentation scores one $\mathrm{SD}$ above the mean (subsequently referred to as the "high-high" group, $N=$ 
13), and those who wanted and obtained segmentation scores one SD below the mean (subsequently referred to as the "low-low" group, $N=14$ ). Results indicated no significant difference in mental health (MCS) based on membership in the high-high good fit group as versus the low-low good fit group, $t(25)=.175, p>.05$. This confirms the polynomial regression coefficient testing findings, indicating that instances of fit along both ends of the continuum (and presumably at all points in between) produce similar mental health outcomes.

Significance testing of the polynomial regression coefficients indicated that the linear coefficient $\left(b_{1}-b_{2}\right)$ along the $P=-S$ line was statistically significant $(F=-3.073, p$ $<.001)$, while the quadratic coefficient along the $P=-S$ line was not $(F=-.466, p>.05)$. This indicates that certain types of misfit are more or less detrimental for mental health than others. Specifically, mental health appears to be slightly worse when preferences exceed supplies than when supplies exceed preferences for segmentation.

Follow-up t-tests were conducted with individuals who experienced two "extremes" of poor fit: those whose segmentation preferences fell one standard deviation or more below the mean but whose workplace segmentation supplies were one standard deviation or more above the mean (subsequently referred to as the "low-high" group, $N=$ 6), and those whose segmentation preferences fell one standard deviation ore more above the mean but whose workplace segmentation supplies were one standard deviation or more below the mean (subsequently referred to as the "high-low" group, $N=12$ ). Results showed no significant difference in mental health composite scores based on the type of poor fit group, $t(16)=1.69, p>.05$. This finding indicates that both extremes on 
the poor fit continuum (and presumably all instances of poor fit between the extremes) produce equal levels of mental health. The conflicting results between the significance testing of the polynomial regressions and the follow-up t-tests may be due to the slightness of the difference in misfit situations, along with the small sample size used to conduct the t-tests.

The research question explored whether high levels of segmentation would be beneficial to health. Results indicated that high-preferences/high-supplies good fit (i.e., those who want and experience high levels of segmentation) was no better for mental health than low-preferences/low-supplies good fit (i.e., those who want and experience low levels of segmentation, also known as high levels of integration), indicating no direct effect of segmentation above and beyond that of congruence on mental health. Highpreferences/low-supplies misfit (i.e., individuals who wanted segmentation but received integration) appears to be worse for mental health than low-preferences/high-supplies (i.e., individuals who wanted integration but received segmentation), although there are conflicting results from multiple tests. This indicates that high levels of actual segmentation may indeed have a somewhat protective effect on mental health in misfit situations, but that the effect is small.

Physical Health. Figures 6 and 7 illustrate the response surface map for congruence and physical health. As is evident from the surface in Figure 6, this is a much different relationship than that of mental health. It appears that physical health is at its peak when boundary supplies are low, in contrast to when they are high. Contrary to 
hypotheses, individuals who both want and achieve highly segmented boundaries (i.e., good fit) appear to have the worst physical health of all.

Figure 6. Response Surface Map of Congruence \& Physical Health

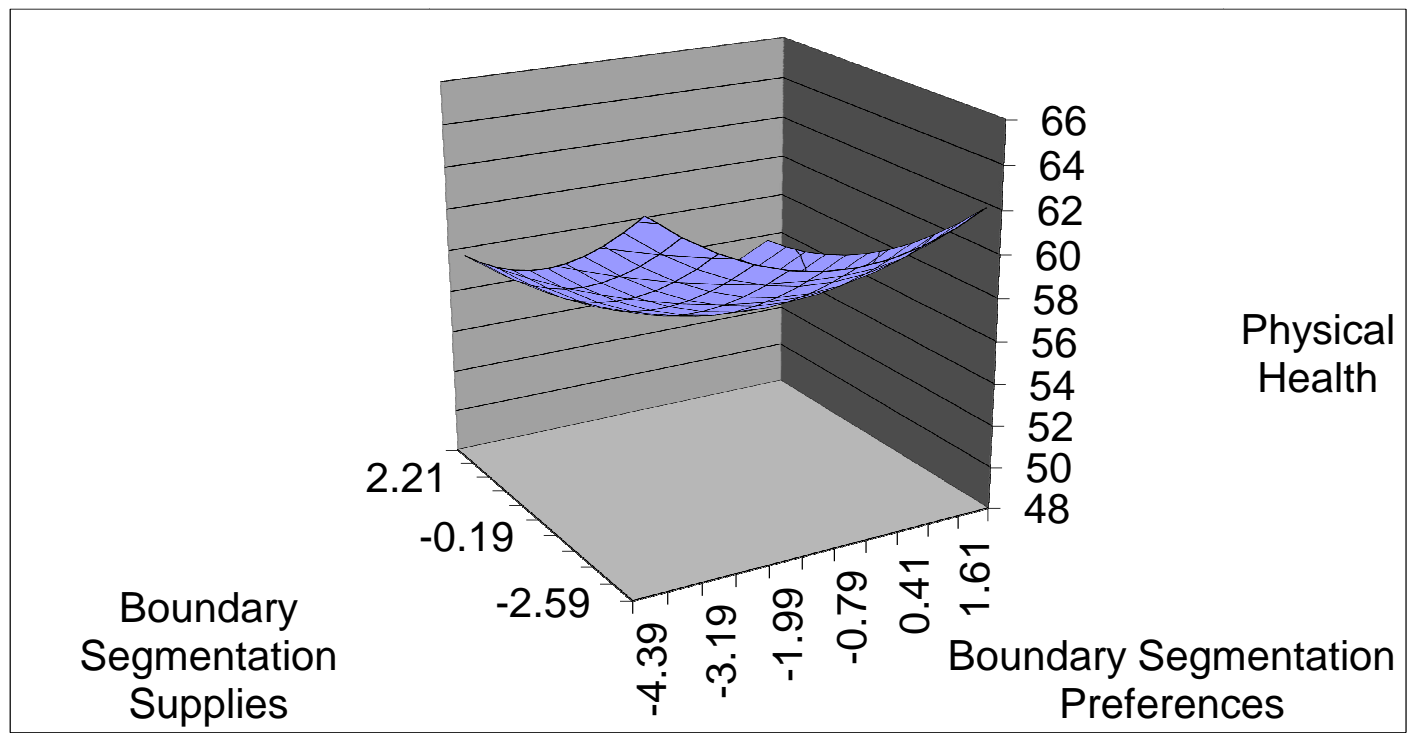

Figure 7. $P=S$ and $P=-S$ Slopes for Congruence $\&$ Physical Health
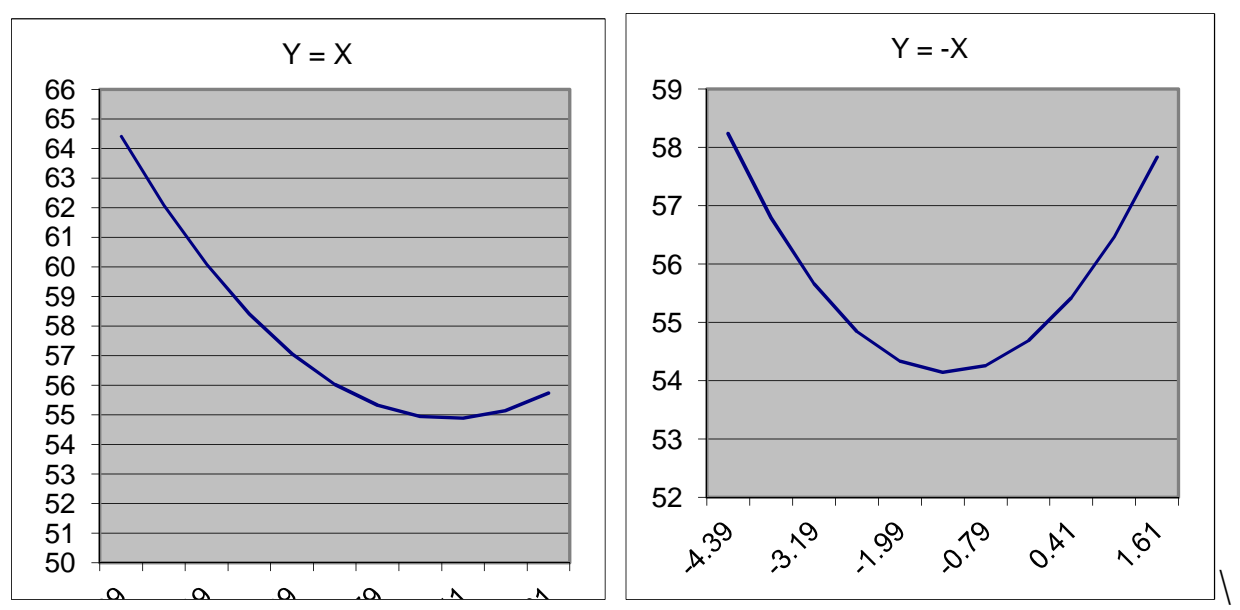
For the outcome of physical health, the quadratic term $\left(b_{3}+b_{4}+b_{5}\right)$ along the $P$ $=S$ line was statistically significant, $F=.448, p<.05$, indicating that not all situations of perfect fit have the same impact on physical health. As is visible in Figure 6, lowpreferences/low-supplies fit (i.e., those who want and receive low levels of segmentation, also known as high levels of integration) appear to result in higher levels of physical health than high-preferences/high-supplies fit (i.e., those who want and receive high levels of segmentation).

Follow-up t-tests were conducted with individuals who experienced two "extremes" of fit: those who wanted and obtained segmentation scores one SD above the mean (subsequently referred to as the "high-high" group, $N=13$ ), and those who wanted and obtained segmentation scores one SD below the mean (subsequently referred to as the "low-low" group, $N=14$ ). Results indicated no significant difference in physical health (PCS) based on membership in the high-high good fit group as versus the low-low good fit group, $t(25)=-.222, p>.05$. The conflicting results between the significance testing for the coefficients and the t-tests may be due to the relatively small difference in the types of good fit, and/or the small sample size utilized in the t-tests.

Significance testing of the polynomial regression coefficients indicated that the linear coefficient $\left(b_{1}-b_{2}\right)$ along the $P=-S$ line was statistically significant $(F=1.133, p$ $<.05)$, while the quadratic coefficient along the $P=-S$ line $\left(b_{3}-b_{4}+b_{5}\right)$ was not $(F=$ $.432, p>.05)$. This indicates that certain types of misfit are more or less "bad" for physical health than others. As illustrated in Figure 6, low-preferences/high-supplies misfit (i.e., those who want integration but receive segmentation) appears to produce 
worse physical health than high-preferences/low-supplies misfit (i.e., those who want segmentation but receive integration).

Follow-up t-tests were conducted with individuals who experienced two "extremes" of poor fit: those whose segmentation preferences fell one standard deviation or more below the mean but whose workplace segmentation supplies were one standard deviation or more above the mean (subsequently referred to as the "low-high" group, $N=$ 6), and those whose segmentation preferences fell one standard deviation ore more above the mean but whose workplace segmentation supplies were one standard deviation or more below the mean (subsequently referred to as the "high-low" group, $N=12$ ).

Results showed no significant difference in physical health composite scores based on the type of poor fit group, $t(16)=-1.94, p>.05$. This finding indicates that both extremes on the poor fit continuum (and presumably all instances of poor fit between the extremes) produce equal levels of physical health. Again, the conflicting results between the coefficient significance testing and the follow-up t-tests may be due in part to either the slightness of the relationship or the small sample size for the t-tests, or some combination of the two.

The research question explored whether high levels of segmentation would be beneficial to health. For the outcome of physical health, it appears that high levels of workplace segmentation may be detrimental, producing slightly worse misfit situations and slightly less beneficial fit situations.

Conflict. Two response surface maps addressed the outcome of work-nonwork conflict: one for work-to-nonwork conflict, and another for nonwork-to-work conflict. 
As coefficient testing found the nonwork-to-work conflict model to be non-significant, it can be found in Appendix K.

Work-to-Nonwork Conflict. Figures 8 and 9 illustrate the response surface map of congruence and work-to-nonwork conflict. The abrupt tilt of the surface in Figure 8 indicates that there is indeed a differential effect of types of misfit on work-to-nonwork conflict. Specifically, individuals who desire highly segmented boundaries but receive a highly integrated work environment suffer from extremely high levels of work-tononwork conflict. Overall, individuals who desire more segmented boundaries are likely to perceive greater conflict from their work upon their home lives, but this trend is especially exacerbated when the work environment does not allow segmentation.

Figure 8. Response Surface Map of Congruence \& Work-to-Nonwork Conflict

Boundary Segmentation Supplies

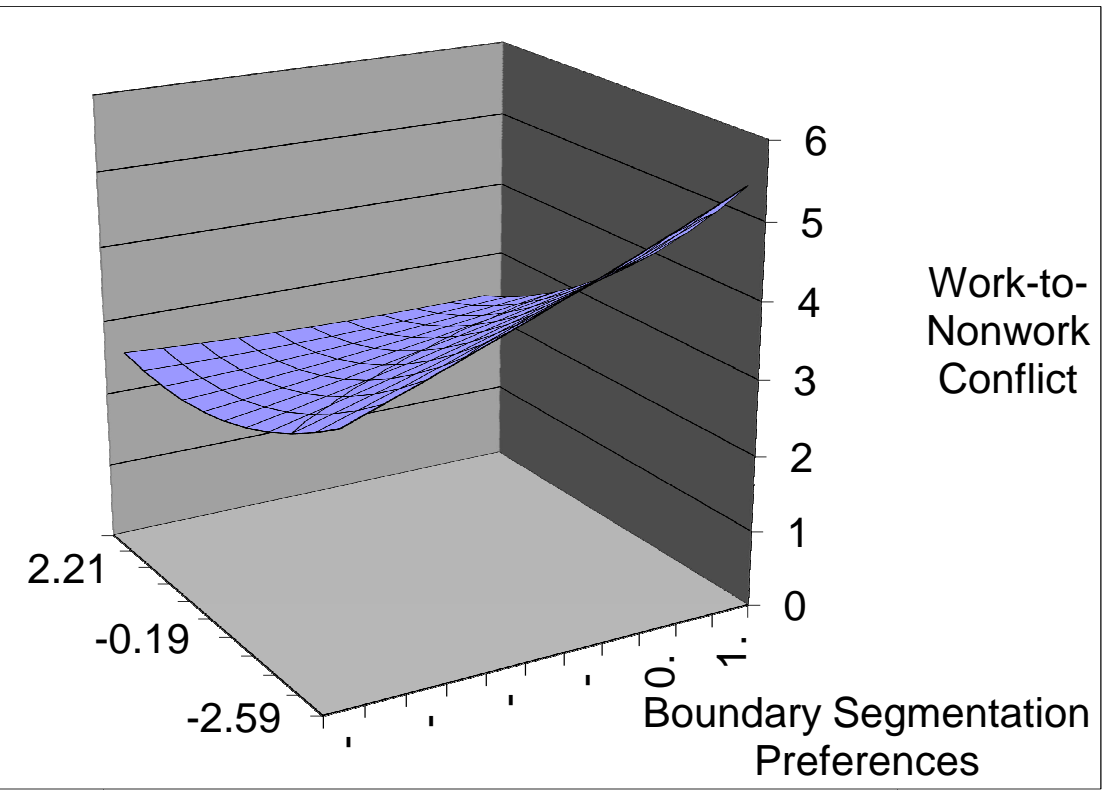


Figure 9. $P=S$ and $P=-S$ Slopes for Congruence \& Work-to-Nonwork Conflict
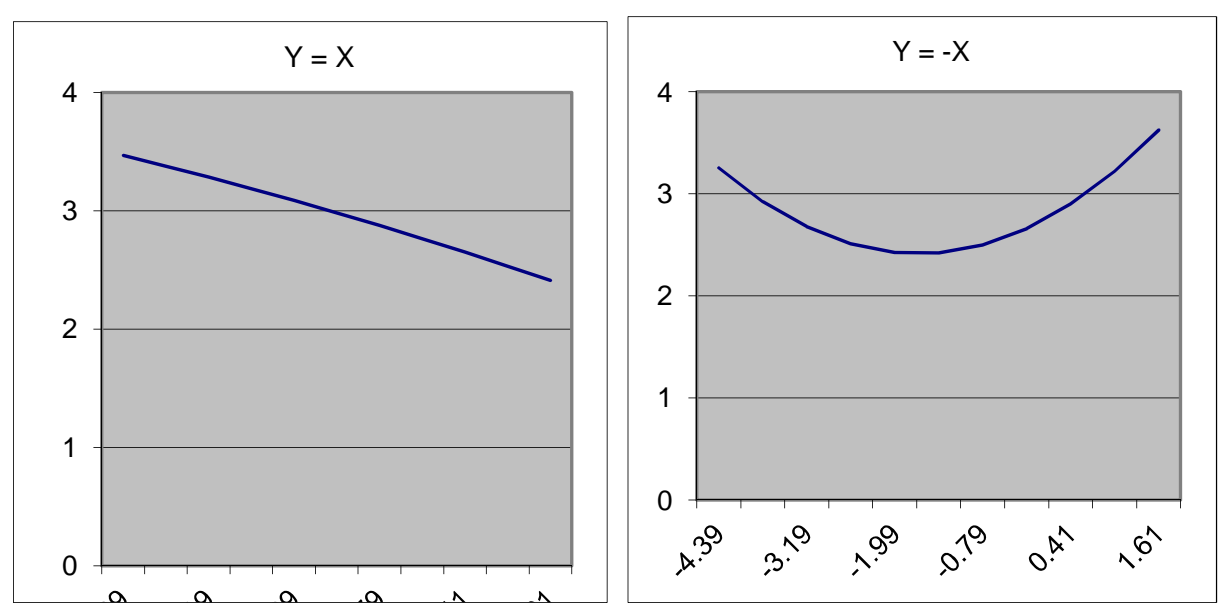

For the outcome of work-to-nonwork conflict, the linear term along the $P=S$ line was statistically significant, $F=-.189, p<.001$, indicating that not all situations of perfect fit have the same impact on work-to-nonwork conflict.

Follow-up t-tests were conducted with individuals who experienced two "extremes" of fit: those who wanted and obtained segmentation scores one SD above the mean (subsequently referred to as the "high-high" group, $N=13$ ), and those who wanted and obtained segmentation scores one SD below the mean (subsequently referred to as the "low-low" group, $N=14$ ). Results indicated a significant difference in work-tononwork conflict based on membership in the high-high good fit group as versus the lowlow good fit group, $t(25)=3.875, p<.01$. Specifically, individuals in the low-low good fit group had significantly higher levels of work-to-nonwork conflict than those in the high-high good fit group ( $M=3.14$ and $M=1.97$, respectively). These findings, along with visual analysis of the response surface maps in Figure 8, indicate that fit situations of low-preferences/low-supplies (i.e., those who want and receive highly integrated work environments) result in significantly higher levels of work-to-nonwork conflict than fit 
situations of high-preferences/high-supplies (i.e., those who want and receive highly segmented work environments).

Significance testing of the polynomial regression coefficients indicated that the linear coefficient along the $P=-S$ line was statistically significant $(F=.376, p<.001)$, as was the quadratic coefficient along the $P=-S$ line $(F=.113, p<.001)$. This indicates that certain types of misfit are more or less "bad" for work-to-nonwork conflict than others.

Follow-up t-tests were conducted with individuals who experienced two "extremes" of poor fit: those whose segmentation preferences fell one standard deviation or more below the mean but whose workplace segmentation supplies were one standard deviation or more above the mean (subsequently referred to as the "low-high" group, $N=$ 6), and those whose segmentation preferences fell one standard deviation ore more above the mean but whose workplace segmentation supplies were one standard deviation or more below the mean (subsequently referred to as the "high-low" group, $N=12$ ). Results showed a statistically significant difference in work-to-nonwork conflict scores based on the type of poor fit group, $t(16)=-6.295, p<.001$. Specifically, individuals in the low preferences-high supplies poor fit group (i.e., individuals who wanted integration but experienced segmentation) experienced significantly less work-to-nonwork conflict than individuals in the high preferences-low supplies poor fit group (i.e., individuals who wanted segmentation but experiences integration; $M=2.50$ and $M=4.28$, respectively). These findings, along with a visual inspection of the response surface map presented in Figure 8, indicate that levels of work-to-nonwork conflict are much higher in the high- 
preferences/low-supplies misfit situation than in the low-preferences/high-supplies type of misfit.

The research question explored whether high levels of segmentation would be beneficial for work-to-nonwork conflict. Results showed that high levels of workplace segmentation contributed to lower levels of work-to-nonwork conflict, both in situations of good fit with preferences and situations of misfit with preferences.

Enhancement. Six response surface maps addressed the outcome of worknonwork enhancement: work-to-nonwork behavior-based enhancement, work-tononwork value-based enhancement, work-to-nonwork affective enhancement, nonworkto-work behavior-based enhancement, nonwork-to-work value-based enhancement, and nonwork-to-work affective enhancement. Due to a lack of significant findings, those pertaining to work-to-nonwork affective enhancement, nonwork-to-work behavior-based enhancement, and nonwork-to-work affective enhancement can be found in Appendix K.

Work-to-Nonwork Behavior-Based Enhancement. Figures 12 and 13 illustrate the influence of congruence on work-to-nonwork behavior-based enhancement. Overall, the surface is relatively flat, while the highest levels of enhancement appear to result from low boundary preferences. 
Figure 10. Response Surface Map of Congruence \& Work-to-Nonwork Behavior-Based Enhancement

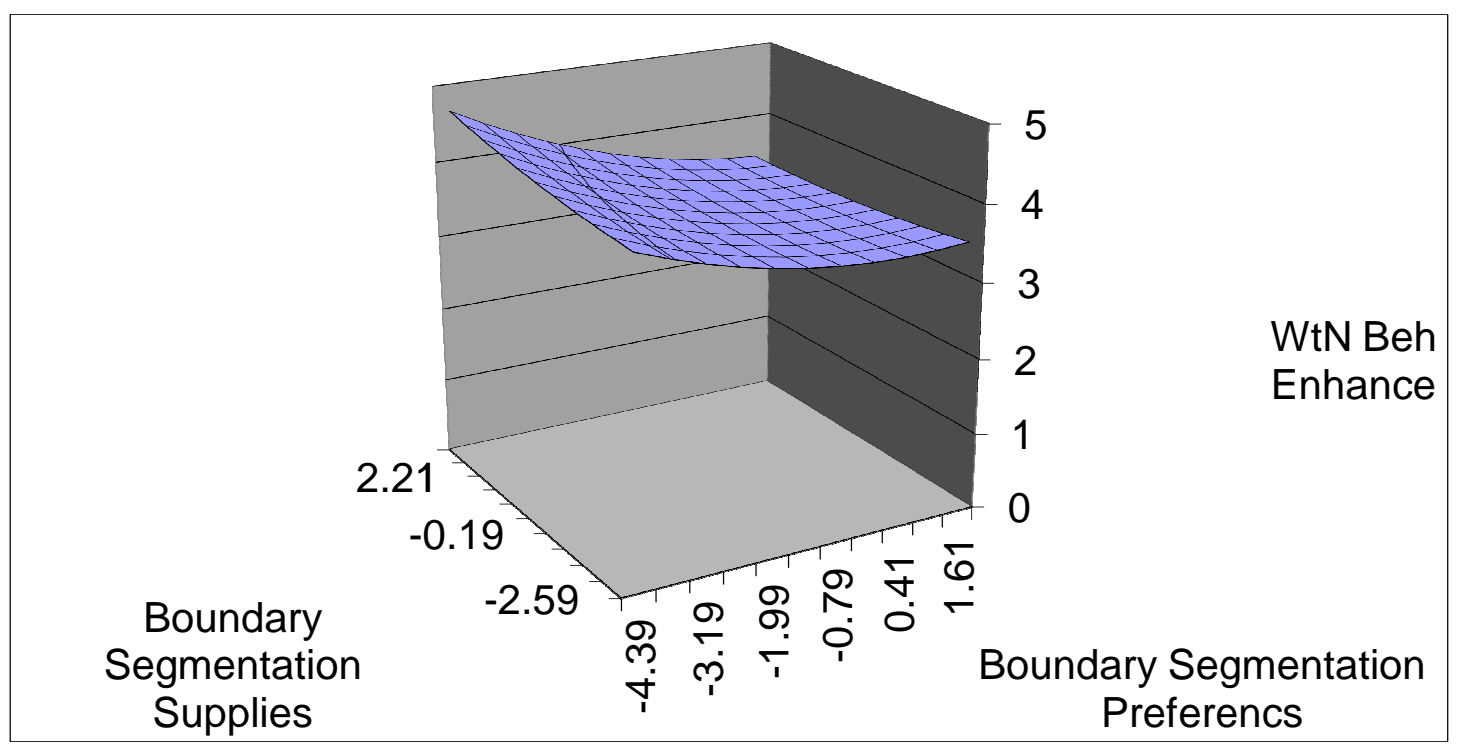

Figure 11. $P=S$ and $P=-S$ Slopes for Congruence \& Work-to-Nonwork BehaviorBased Enhancement
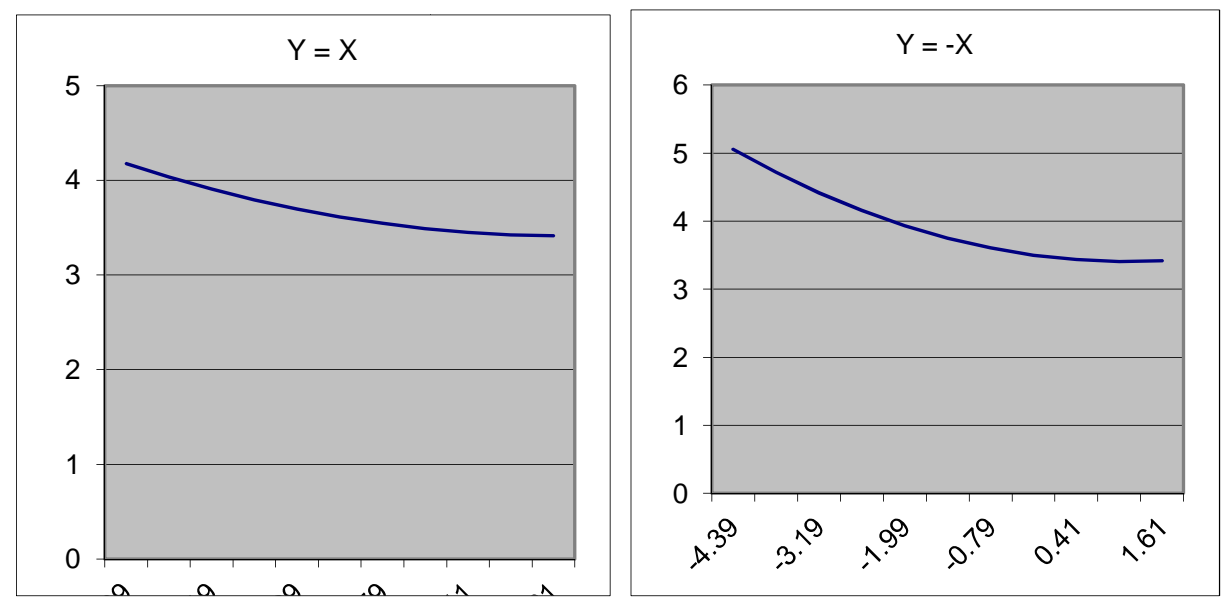

For the outcome of work-to-nonwork behavior-based enhancement, both coefficients along the $P=S$ line were nonsignificant, indicating that all instances of 
perfect fit have a comparable influence on work-to-nonwork behavior-based enhancement, regardless of where that "perfect fit" falls on the continuum.

Follow-up t-tests were conducted with individuals who experienced two "extremes" of fit: those who wanted and obtained segmentation scores one SD above the mean (subsequently referred to as the "high-high" group, $N=13$ ), and those who wants and obtained segmentation scores one SD below the mean (subsequently referred to as the "low-low" group, $N=14$ ). Results showed no significant difference in work-tononwork behavior-based enhancement scores based on the type of good fit group, $t(25)=$ $1.48, p>.05$. This finding confirms that both extremes on the good fit continuum (and presumably all instances of fit between the extremes) produce equal levels of work-tononwork behavior-based enhancement.

Significance testing of the polynomial regression coefficients along the $P=-S$ line demonstrated a different pattern, such that the linear coefficient was negative and statistically significant, $F=-.122, p<.05$, while the quadratic coefficient was not statistically significant $(F=.053, p>.05)$.

Follow-up t-tests were conducted with individuals who experienced two "extremes" of poor fit: those whose segmentation preferences fell one standard deviation or more below the mean but whose workplace segmentation supplies were one standard deviation or more above the mean (subsequently referred to as the "low-high" group, $N=$ 6), and those whose segmentation preferences fell one standard deviation ore more above the mean but whose workplace segmentation supplies were one standard deviation or more below the mean (subsequently referred to as the "high-low" group, $N=12$ ). 
Results showed a statistically significant difference in work-to-nonwork behavior-based enhancement scores based on the type of poor fit group, $t(16)=3.163, p<.01$. Specifically, individuals in the low-preferences/high-supplies group (i.e., individuals who wanted integration but received segmentation) have significantly higher levels of workto-nonwork behavior-based enhancement than individuals in the high-preferences/lowsupplies group (i.e., individuals who wanted segmentation but received integration; $M=$ 4.67 and $M=3.33$, respectively). These results, along with visual inspection of the response surface map in Figure 12, indicate that individuals who prefer integrated worknonwork boundaries but receive segmented work-nonwork boundary supplies have greater levels of work-to-nonwork behavior-based enhancement than their highpreferences/low-supplies counterparts.

The research question explored whether high levels of workplace segmentation would be beneficial for enhancement. Results indicate that segmentation level does not influence how good fit influences work-to-nonwork behavior-based enhancement (i.e., all situations of good fit have equal influence on this facet of enhancement, regardless of where they fall on the segmentation spectrum), but that misfit situations that involve high levels of provided segmentation do result in more positive outcomes than misfit situations with low levels of provided segmentation.

Work-to-Nonwork Value-Based Enhancement. Figures 14 and 15 illustrate the response surface map of congruence and work-to-nonwork value-based enhancement. The direction of the tilt on the map (from side to side rather than from front to back) indicates that, similar to the results for work-to-nonwork behavior-based enhancement, 
boundary preferences have a greater impact on work-to-nonwork value-based enhancement than boundary supplies. Individuals with low boundary preferences (i.e., prefer high integration) appear to have slightly higher levels of value-based enhancement compared to those who prefer highly segmented boundaries.

Figure 12. Response Surface Map of Congruence \& Work-to-Nonwork Value-Based Enhancement

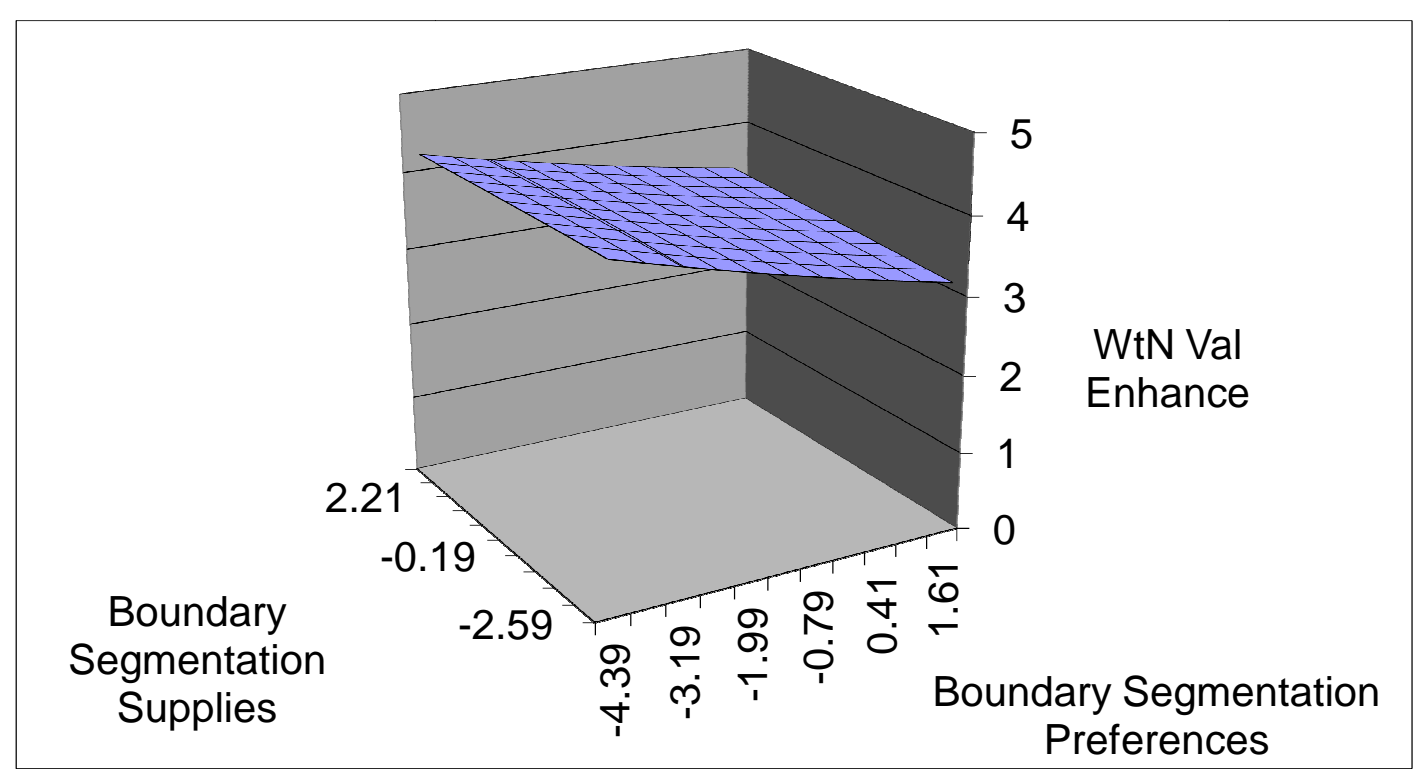


Figure 13. $P=S$ and $P=-S$ Slopes for Congruence \& Work-to-Nonwork Value-Based Enhancement
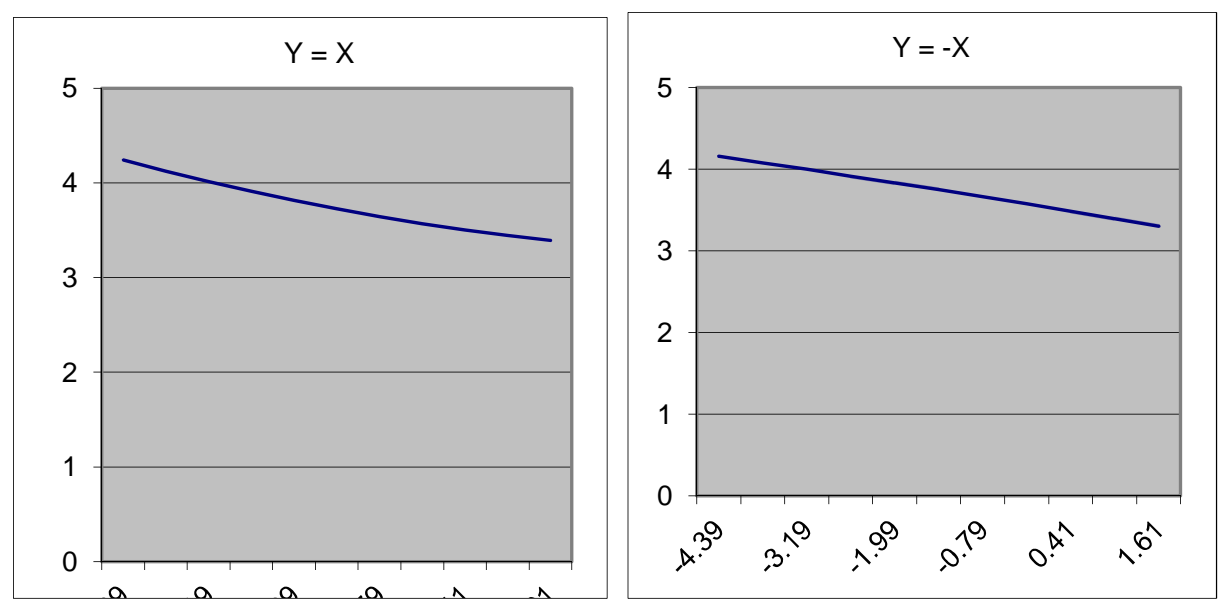

For the outcome of work-to-nonwork value-based enhancement, the linear coefficient along the $P=S$ line was negative and statistically significant, $F=-.114, p<$ .05. This indicates that individuals with good fit who want and receive an integrated workplace have more work-to-nonwork value-based enhancement than individuals with good fit who want and receive a segmented workplace.

Follow-up t-tests were conducted with individuals who experienced two "extremes" of fit: those who wanted and obtained segmentation scores one SD above the mean (subsequently referred to as the "high-high" group, $N=13$ ), and those who wanted and obtained segmentation scores one SD below the mean (subsequently referred to as the "low-low" group, $N=14$ ). Results showed a significant difference in work-tononwork value-based enhancement scores based on the type of good fit group, $t(25)=$ $2.42, p<.05$. Specifically, individuals on the low-low end of the good fit continuum (i.e., those who want and receive integration) have significantly higher levels of work-tononwork value-based enhancement than individual on the high-high end of the good fit 
continuum (i.e., individuals who want and receive segmentation; $M=4.26$ and $M=3.31$, respectively).

Significance testing of the polynomial regression coefficients along the $P=-S$ line demonstrated that the linear coefficient was negative and statistically significant, $F=$ $-.148, p<.01$, while the quadratic coefficient was not statistically significant $(F=-.002$, $p>.05)$.

Follow-up t-tests were conducted with individuals who experienced two "extremes" of poor fit: those whose segmentation preferences fell one standard deviation or more below the mean but whose workplace segmentation supplies were one standard deviation or more above the mean (subsequently referred to as the "low-high" group, $N=$ 6), and those whose segmentation preferences fell one standard deviation ore more above the mean but whose workplace segmentation supplies were one standard deviation or more below the mean (subsequently referred to as the "high-low" group, $N=12$ ). Results showed a statistically significant difference in work-to-nonwork value-based enhancement scores based on the type of poor fit group, $t(16)=3.84, p<.01$. Specifically, individuals in the low-preferences/high-supplies group (i.e., those who want integration but receive segmentation) have significantly higher levels of work-tononwork value-based enhancement than those in the high-preferences/low-supplies group (i.e., those who want segmentation but receive integration; $M=4.33$ and $M=2.61$, respectively). These results, along with visual inspection of Figure 14, indicate that individuals who prefer integration but receive segmentation have higher levels of work- 
to-nonwork value-based enhancement than misfit situations with high-preferences/lowsupplies.

The research question explored whether high levels of workplace segmentation would be beneficial for enhancement. These results indicate that preferences, not actual levels of segmentation, have greater predictive power over levels of enhancement, such that individuals with a preference for integration experience higher levels of work-tononwork value-based enhancement.

Nonwork-to-Work Value-Based Enhancement. Figures 20 and 21 show the influence of congruence on nonwork-to-work value-based enhancement. Given that the surface is relatively flat, it appears that boundary congruence does not have much of an effect on nonwork-to-work value-based enhancement.

Figure 14. Response Surface Map of Congruence \& Nonwork-to-Work Value-Based Enhancement

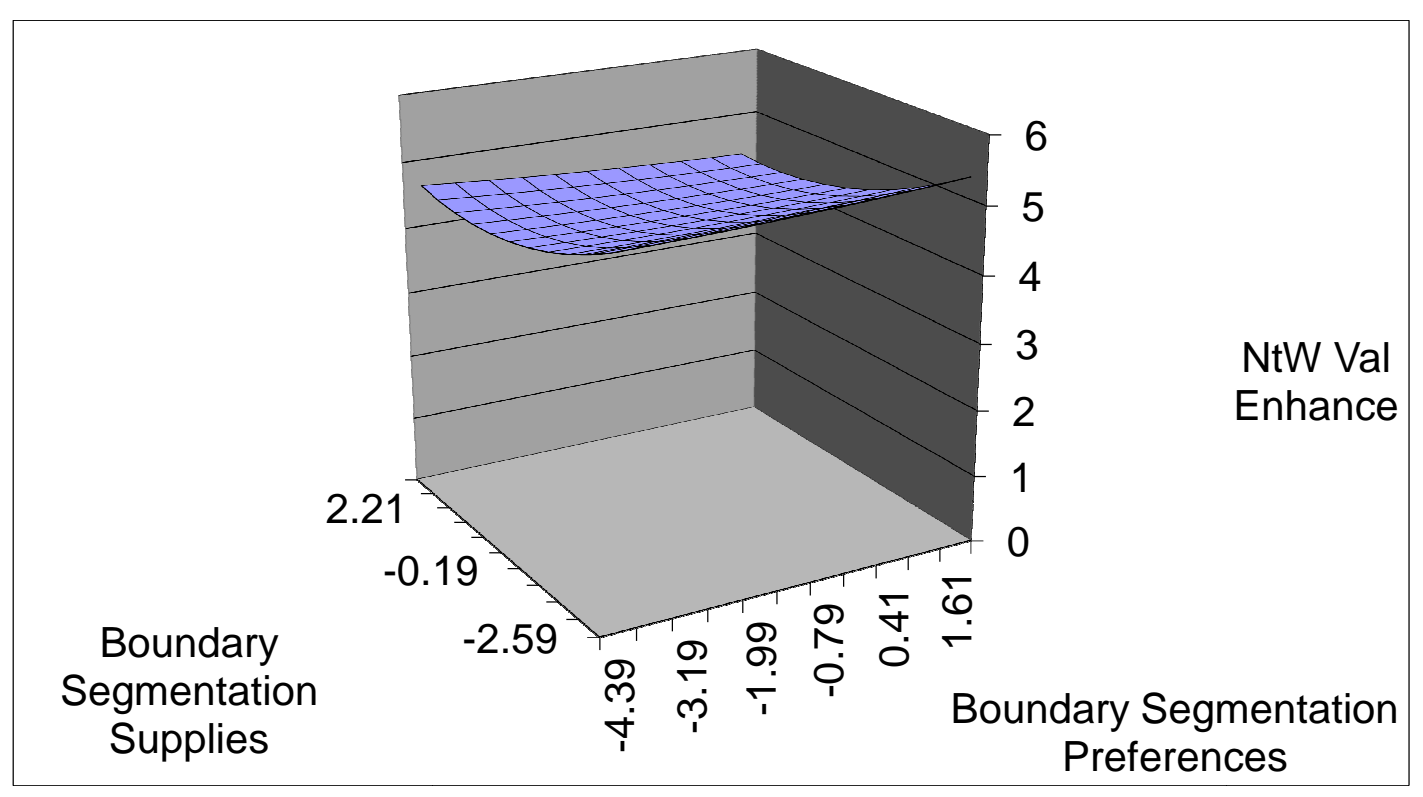


Figure 15. $P=S$ and $P=-S$ Slopes for Congruence \& Nonwork-to-Work Value-Based Enhancement
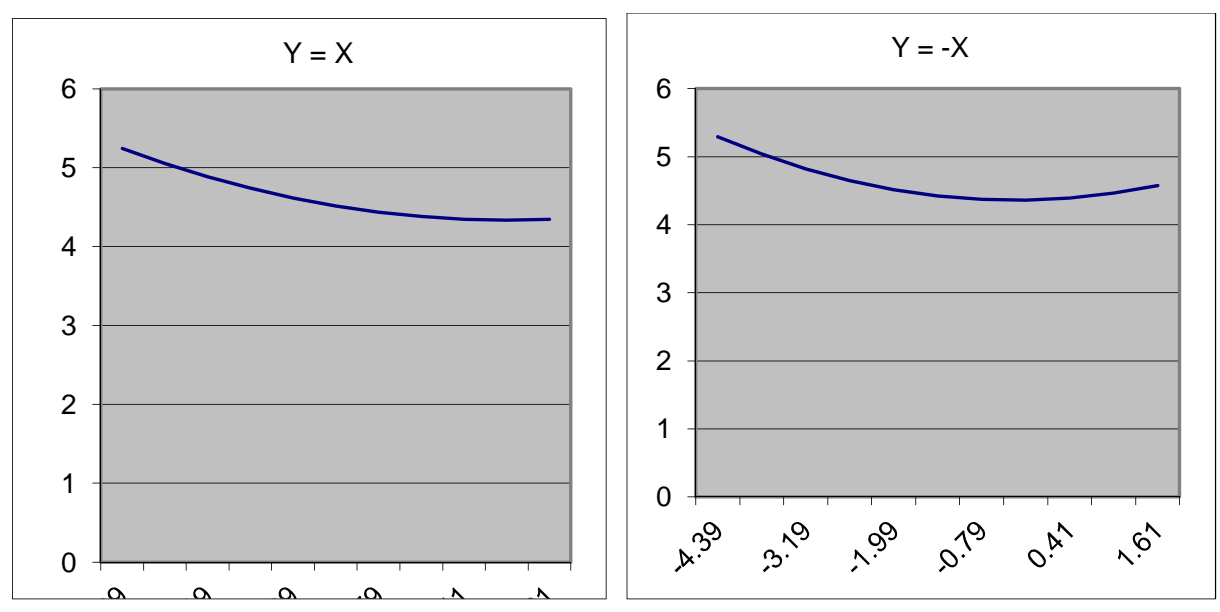

For the outcome of nonwork-to-work value-based enhancement, both coefficients along the $P=S$ line were nonsignificant, indicating that all instances of perfect fit have a comparable influence on nonwork-to-work value-based enhancement, regardless of where that "perfect fit" falls on the continuum.

Follow-up t-tests were conducted with individuals who experienced two "extremes" of fit: those who wanted and obtained segmentation scores one SD above the mean (subsequently referred to as the "high-high" group, $N=13$ ), and those who wanted and obtained segmentation scores one SD below the mean (subsequently referred to as the "low-low" group, $N=14$ ). Results showed no significant difference in nonwork-towork value-based enhancement scores based on the type of good fit group, $t(25)=.583, p$ $>.05$. This finding confirms that both extremes on the good fit continuum (and presumably all instances of fit between the extremes) produce equal levels of nonworkto-work value-based enhancement. 
For the outcome of nonwork-to-work value-based enhancement, the linear coefficient was not significant $(F=.039, p>.05)$, while the quadratic coefficient was positive and significant $(F=.057, p<.01)$. Thus, it appears that for the outcome of nonwork-to-work value-based enhancement, all instances of misfit are equally bad. Examining the $P=S$ and $P=-S$ lines together, it appears that this outcome exhibits the tenets of boundary theory perfectly.

Follow-up t-tests were conducted with individuals who experienced two "extremes" of poor fit: those whose segmentation preferences fell one standard deviation or more below the mean but whose workplace segmentation supplies were one standard deviation or more above the mean (subsequently referred to as the "low-high" group, $N=$ 6), and those whose segmentation preferences fell one standard deviation ore more above the mean but whose workplace segmentation supplies were one standard deviation or more below the mean (subsequently referred to as the "high-low" group, $N=12$ ).

Results showed no significant differences in nonwork-to-work value-based enhancement scores based on the type of poor fit group, $t(16)=-.74, p>.05$. This finding indicates that both extremes on the poor fit continuum (and presumably all instances of poor fit between the extremes) produce equal levels of nonwork-to-work value-based enhancement.

The research question explored whether high levels of workplace segmentation would be beneficial for enhancement. Misfit between boundary preferences and boundary supplies somewhat lowers nonwork-to-work value-based enhancement, but this is not a 
very large effect. Rather, it appears that neither preferences nor supplies make much of a difference in this facet of enhancement.

\section{Qualitative Analyses}

Coding showed a variety of interesting patterns in the qualitative data. See Appendix $\mathrm{H}$ for the codebook used to code the open-ended responses. The codes that emerged rarely directly mapped on to either the hypotheses or the research question, but instead, gave context to the work-nonwork interface.

Descriptions. Many responses were simple statements pertaining to their workplace. For example, "I own my own business", and "I work from home 2 days a week" and "I work at an elementary school". These statements seemed to indicate that the respondents felt it important to disclose these facts, but without further comment on how those facts influenced their work-nonwork balance or well-being, they are difficult to interpret. To avoid making inferences, these were coded simply as Descriptions.

Boundary Management Strategies. Many responses described the individual's current or ideal boundary management strategy. These responses, ranging from highly integrated to highly segmented, were coded as Boundary Management Strategies. Many responses illustrated how some individuals enjoy integration of work and nonwork. For example, "Because I think about work when I'm at home it gives me greater flexibility with my work schedule, which improves my home life". Another respondent stated, "I sometimes enjoy working through work problems at home, usually on the computer". Several specified, however, that they enjoyed integration when it was their choice. For example, "I like to think about work at home but only on occasion and on my own terms. 
I do not want to be expected to respond to work issues during my personal home time", and "I don't mind working on work things at home when it is my choice to do so, but I am very unhappy when others cause me to NEED to work on things at home."

Conversely, many other responses indicated that participants preferred segmentation: "In my particular job, I am generally able to leave my work at work, which is what I prefer. I do not tend to bring work home with me except in very unusual circumstances". Another respondent stated, "I deliberately keep the line separate between work and home. I am a contract worker paid hourly, so I am only expected to work 40 hours/week and then go home".

These examples demonstrate the argument made in the Introduction of this dissertation: boundary preferences range widely across individuals, and what seems perfect for one individual may be extremely difficult for another individual. This gives credibility to the quantitative findings that preferred boundary strategies exist at both ends of the spectrum presented, and everywhere in between.

Positive Examples. Many comments were coded under the umbrella of Positive Examples. These stories shared how work-nonwork balance was supported for participants. Some of these described how the organization exhibited a positive culture and supported their employees' efforts to balance their demands. For example, "As the organization I work for has a mandate about being 'family centred' in working with our clients, that philosophy is also applied to its employees allowing and more importantly encouraging employees to leave work matters at work." Another wrote, 
"My organization is very flexible and my boss only cares that my work gets completed on time. He doesn't necessarily care when it happens. Employees are able to take time during the day for doctor appointments, events at kids' school, etc. very easily. The only time we have to be at the office or client site is when we have a scheduled meeting; otherwise, it is up to us to get tasks completed on time. One colleague has small kids and she works at home. During the afternoon, she picks her girls up from school, helps them with their homework, makes dinner and such. After that, she will go back to her work for a few hours."

Other examples of positive work-nonwork balance included individual descriptions of the type of work-nonwork enhancement they experienced. For example, "I used to be very shy, but going into a career that involved interfacing with the public built my interpersonal skills and convinced me I was actually good with people. Also, being a parent has proven to be good practice for being a supervisor/manager". Finally, some responses illustrated conditions of good fit between the person's boundary management preferences and the boundary supplies offered by the organization. One respondent said, "My position is flexible and able to be performed remotely - so I can do work from a hospital room if I need to - fits my needs perfectly. I can still go to the track meet and get my work done. Also I am devoted to children and it is a child care company - great fit!”

The responses that fell into this category gave credence to the assertions made in the introduction of this paper that work-nonwork enhancement does indeed occur for many individuals, and that they respond favorably to this. It also illustrated that 
individuals experiencing good fit between their work and home lives do exist, and that not everyone has a work environment that they dislike or need to take breaks from.

Negative Examples. Alternatively, another group of responses were categorized as Negative Examples. These described instances where the employee's efforts at worknonwork balance were unsuccessful. Some of these responses described a culture that did not support balance, for example, "There is a culture of overwork where I am employed." Others described that while there were policies in place to support worknonwork balance, the actual day-to-day practices did not support these policies: "There is a stated desire for employees to have work and home life but the reality of the behaviors do not match in that there is an expectation to "'get the work done"" regardless of how that interacts with home life."

Some of the Negative Examples described examples of work-nonwork conflict. For example, "I value my personal life highly over my work life, so my personal values and activities are almost always a distraction to my work. While I gain valuable skills, build productive relationships and even enjoy my work, being at work at all (even though I work for a great non-profit) is almost always in direct conflict with my priorities of nurturing my family and working to improve my community". Another respondent stated, "My work causes me to make sacrifices to my personal life. It's frustrating that my supervisors are unwilling to help alleviate the situation."

Other quotes illustrated examples of poor fit between the individual's boundary preferences and the organization's boundary supplies. This occurred both in terms of high segmentation: "My organization is more adherent to disassociating from work when 
away then I might be" and high integration: "It is encouraged to monitor email at home, which I don't like to do". These two examples illustrate the concept discussed in the Introduction of this paper-poor fit can occur in either direction. It also serves to bolster the confidence in the quantitative findings by substantiating the idea that this dataset contains both types of misfit, enabling examination of the research question.

One of the gaps this study sought to fill was examining work-nonwork conflict instead of work-family conflict. Thus, while some of the Negative Examples category contained examples of family-related home demands ("For the past four weeks my mother has been hospitalized", and, "Having a new child makes work even more stressful"), there were also plenty of examples of non-family related home demands. For example, "We have 4 new playful and biting puppies at home so stress level is higher" and "In the last month I just bought a house so my home life has taken up an extraordinary amount of time". Other duties that were non-family related included studying for the bar, moving, graduate school, and volunteer activities, among others.

These qualitative results confirmed that home life concerns other than those that are strictly family-related can make a large impact on work-life balance, and that this study was successful at reaching individuals who had non-family related home-life sources of stress and strain.

Challenges to Balance. What factors make preferred boundary management style particularly difficult? The code Challenges to Balance illustrated some factors that aggravated work-nonwork conflict or made it particularly hard to maintain balance. Intense demands and lowered resources were cited as a challenge: 
"I am in a sales job and am in the field for 8-10 hours Monday-Friday (driving, calling on customers, etc.). We are expected to do 'office' work at home in the evenings and weekends. The tasks that we need to complete are often assigned by someone who is sitting in an office all day and has no idea how much time these tasks require.

Consequently, I sometimes work 12 hours or more per day and often on the weekends. I find it unfair and it is difficult to have any sort of balance".

Commuting seemed to be another recurring challenge: "My awful commute is more to blame for my craziness (too tired when I come home) fighting traffic both ways." Similarly, individuals who needed to travel for work also found it challenging: "I travel extensively and I find it more difficult to balance work/family when I am in a different time zone".

Those who desired segmentation found that increasing use of technology made their preferred boundary management style extremely difficult. For example, "It is not the workplace which causes work to interfere with home. It's the world and communication. We receive email messages from vendors, regulators and others at all hours of the day and night. Unless one carries two communication devices, it is impossible not to have the 2 worlds overlap". Similarly, "Having Smartphone connection to the e-mail server and VPN access to my workstation has made disconnecting from work more difficult. These tools allow me to work while out of town on business, but also makes home interruptions much more frequent".

The responses in this category are excellent indicators of factors that should be taken into account in future studies regarding work-nonwork balance. While some 
research is already being conducted on the topics of commuting and technology and their influence on work-nonwork balance, it is clear that these are important factors for some individuals and deserve continued attention.

It Depends. Many responses gave a response categorized as It Depends. Their statements indicated that their boundary management styles and/or their work-nonwork balance was due to specific factors. Interestingly, these specific factors varied (e.g., on their particular occupation, organization, department, or position) and had the power to either make boundary management more segmented or more integrated, seemingly against the individual's desire.

On the macro level, several responses indicated that the participant's chosen occupation or career allowed or did not allow certain boundary management styles. Generally, many responses indicated that in academia, boundary segmentation was simply not possible: "College professors never stop working. But we set our own hours! (sometimes)". Similarly, "I think an academic job is inherently different - and it's not uncommon or necessarily bad to have some associated work or mental processing going on while not at work". On the flip side, several responses indicated that many health care professionals felt that segmentation was an aspect of the career, not a choice: "I work at the VA so I don't/can't have email access at home, hence I can't bring my work home" and, "As an RN, I am legally prohibited from discussing work in any detail with anyone outside the work place". These responses demonstrate that work-nonwork balance may look very different in different occupations, and that occupation-specific studies may be warranted. 
On the meso level, responses indicated that within a single organization, line/hourly workers were able to segment while management/salaried employees were forced to integrate: "I think this is a function of how high 'up' you are in the company. The organization very much values work and life, as espoused by the same managers who have far less work-life balance than those they manage". Similarly, "I have the benefit of working an entry level job, so have less responsibility to bleed into home life. I know my coworkers and supervisors frequently respond to email from home and work on projects at home as well". These responses indicate that future research would do well to design a nested study of work-nonwork balance within a single organization to quantify the suppositions made by these participants.

Finally, on the micro level, some indicated that boundary management strategies were allowed or not allowed by individual departments or supervisors. For example, "I think it depends on the department. My department is very supportive of work-life balance" and, "My immediate supervisor is very supportive and allows freedom and flexibility. It is not the policy of the organization".

Individual Differences. The It Depends code illustrated how people felt that their boundary management strategies and work-nonwork balance were influenced by external forces, such as their occupation, organization, or supervisor. In contrast, the code Individual Differences illustrated that some individuals perceive work-nonwork balance and boundary management strategies to be an individual difference, a choice, and/or something to be learned over time. For example, one individual felt that the organization had little to no influence over his/her work-nonwork balance, and that the 
onus of creating balance lay solely in the individual's hands: "These are all personal choices, it's not up to my employer or work to create balance for me. I am an adult and make my own choices". Similarly, another stated, "I think how much you can leave work at work is more a function of the individual than the work place".

Other responses within the Individual Difference code indicated that to them, boundary management strategies are learned and perfected over time. For example, "It took several years and being put in antidepressants twice before I learned how to mostly balance my work and home life. It is a skill that too few people have learned and far too many companies don't value employees who separate work and home life. I am lucky that my employer mostly supports efforts of employees to have a separation between work and home." Another stated, "I have built skills over the years around setting strong work/life boundaries, and handling overtime and/or excessively stressful work demands when they occur. I did not develop these skills until my mid-thirties (I've been in the workforce since before college)".

Lastly, some responses within the Individual Differences code described some of the conscious decisions participants had made in an attempt to gain work-nonwork balance and to take control of their boundary management style. For example, "I often choose to work at home in the evenings in order to have more daytime hours with my family", and, "The reason why I have crafted a home based and sales business is so that I can take care of my health with exercise and healthy eating". One respondent stated, "I consciously took a position of less income and prestige that allows me to keep my work life separate from my home life". 
The responses in this category, especially when compared to those in the $I t$ Depends category, demonstrate that the construct of boundary management is not widely agreed upon, and that it likely means different things to different people. Thus, some participants likely responded to the survey thinking of boundary management as an individual difference, under the control of an individual, while others felt that it was thrust upon them by a higher power. The responses of these two groups of participants are likely quite different. Future research should explore the concept of a boundary management locus of control and it's relation to work-nonwork balance.

Exhaustion. While the health outcome variables in this study examined physical and mental health in general, some responses noted that their primary health complaint was that they were exhausted. These responses were simply coded Exhaustion. For example, "I am healthy but there are many times when I am too tired from being on my feet all day to feel motivated to get out and get other things (with a physical component to them) done, that I used to do much more regularly". Another stated, "My leisure time is so rare that it is usually spent sleeping/relaxing". These responses may indicate that the participants did not feel the topic of exhaustion was adequately covered in the health outcome measure utilized in this dissertation, and thus felt the need to explain further. Future research may potentially benefit by examining the outcome of exhaustion separately from other health outcomes, given its prevalence here.

Alternative Perspectives. Several of the open-ended responses were somewhat counter to dominant OHP theories, and thus, were coded as Alternative Perspectives. While scarcity theory focuses on the demands of the workplace, and enhancement theory 
focuses on the positive values, behaviors, skills, and affect that can stem from the workplace, very little literature covers the concept of work as a respite from home life. Generally, such as in the recovery literature, we consider that home life is where one recovers from work. However, some of the open-ended responses indicated that work can also serve as a recovery experience from the demands of non-work life. For example: "Work is often my escape from a chaotic home life" and "Currently outside work (personal life) is more challenging than worklife. Work can be a nice, independent break from home life".

Examining the results with this perspective shows that the measure of boundary management is flawed: it is uni-directional. The preferences scale asks whether individuals prefer to keep work out of their home life, and the supplies sub-scale asks whether their workplace allows them to keep work out of their home life. There is no parallel set of sub-scales asking if they prefer or are able to keep their home life out of their work life (Kreiner, 2006). This implies that work is the encroacher, and home life does not suffer such problems that individuals would want to "get away" from. Similarly, the recovery from work scale (Sonnentag \& Fritz, 2007) implies that work is the demanding role, and home life is the arena where recovery is possible. For individuals whose responses fell into this Alternative Perspectives category, the opposite may be true. Thus, the uni-directionality of some of the scales utilized in the present dissertation may have restricted the results from showing the "whole" picture.

The next Alternative Perspective code pertains to enhancement theory. Enhancement theory posits that positive states in one domain transfer to the other domain 
in a beneficial manner (Marks, 1977). However, some open-ended responses indicated the opposite. "The better my life is outside of work, the more I realize how much I hate my job," said one participant, while another stated, "Being in a positive mood at work sometimes makes me sad about my home life, because of the jarring contrast there.” Another attributed this to her family: "I have a young daughter so when I'm happy at home, I wish I was with her while at work". It appears that happiness does not spill over to opposite domains for everyone. This perspective is an interesting one, not covered in either scarcity theory nor enhancement theory (Goode, 1960; Marks, 1977). These responses indicate that perhaps additional theories regarding crossover between domains should be pursued.

Timing. Several responses pertained to the timing of the survey. Some responses indicated that current issues might influence responses, such as: "Just a reminder that some of your respondents may have spent the past month or a good part of it on vacation" and "Stated values are quite a different thing than stability of employment relationship. The economic climate and landscape change everything". Others described how the timing particularly influenced their responses, such as, "Our grey, wet spring has not helped one, single bit! It has kept me indoors, the grey days can be very gloomy". Others mentioned how specific unusual circumstances have influenced their health that were not de rigueur: "I had surgery 4 weeks ago and am still recovering, so my answers to these questions are not typical of any 4 week period". Other examples: "I have been pregnant (just gave birth this week)" and "had a recent heart attack". 
Most of the responses in this category serve to emphasize the basis of scientific rigor: this study should be replicated at other times and other places to minimize the amount that these timing issues influence our understanding of the underlying relationships. Only by repetition of research will we be able to truly understand the "real story".

Survey Feedback. Lastly, several comments gave feedback on the survey construction itself. For example, some suggested topics to include on future surveys: "I think asking about exercise habits would lend more information about frame of mind. People that exercise, in my opinion, are happier and more balanced". Others critiqued various items on the survey: "The items in your scale are SO similar. Are you really capturing different dimensions...cant this be shorter??" Others encouraged the study of the topic: "Very well done and I hope I hear the results". 


\section{Chapter 6}

\section{Discussion}

\section{Overall Findings}

Hypothesis 1a, positing a negative relationship between work-to-nonwork conflict and health, was partially supported: mental health, but not physical health, was significantly and negatively related to work-to-nonwork conflict. Hypothesis $1 \mathrm{~b}$, which posited a negative relationship between nonwork-to-work conflict and health, was also partially supported. Once again, conflict was significantly and negatively related to mental health but not physical health.

Hypothesis 2 proposed a positive relationship between work-nonwork enhancement and health, such that both directions and all types of enhancement should have a positive influence on health. Hypothesis $2 \mathrm{c}$ was partially supported; work-tononwork affective enhancement had a significant positive effect on physical health, but not mental health. Similarly, Hypothesis $2 \mathrm{f}$ was partially supported; nonwork-to-work affective enhancement had a significant positive effect on physical health, but not mental health. All of the other types and directions of enhancement did not appear to have a significant effect on mental or physical health, providing no support for Hypotheses 2a, $2 \mathrm{~b}, 2 \mathrm{~d}$, or $2 \mathrm{e}$.

Hypothesis 3a proposed that congruence between boundary preferences and boundary supplies would have a significant and positive relationship on mental health. This relationship was fully supported. Hypothesis $3 \mathrm{~b}$ proposed that congruence would have a similar positive relationship with physical health, however, this hypothesis was 
not supported. In fact, 10 of the 12 models testing this hypothesis found a significant and opposite relationship, such that high levels of congruence between boundary supplies and boundary preferences was significantly related to lowered physical health.

Hypothesis 4 proposed that both types of work-nonwork conflict would serve as mediators between the congruence-health relationship. Results partially supported Hypothesis 4a, showing that work-to-nonwork conflict did mediate the relationship between congruence and mental health, but not between congruence and physical health. There was no support for Hypothesis $4 \mathrm{~b}$ that proposed nonwork-to-work conflict as a mediator of congruence and health.

Hypothesis 5 proposed that all six types of enhancement would serve as mediators between congruence and health. This was not supported in any of the models.

The research question explored whether high levels of experienced segmentation would be more beneficial for outcomes than high levels of integration. Results were mixed regarding the research question. For the outcomes of mental health, work-tononwork value-based enhancement, and nonwork-to-work value-based enhancement, segmentation level did not appear to have an influence of its own on outcomes, other than that related to congruence. In contrast, segmentation level appeared to be beneficial for work-to-nonwork conflict as well as work-to-nonwork behavior-based enhancement, such that higher levels of overall segmentation led to lower levels of conflict and higher levels of enhancement. Finally, some evidence showed that high levels of segmentation may be bad for physical health, rather than beneficial, as the research question suggested. Overall, the results indicate that congruence, rather than simple level of segmentation, is 
a stronger predictor of outcomes. Additionally, it appears that while segmented boundaries may be beneficial for some outcomes, findings are non-significant or tending towards the opposite end for other outcomes, and thus, more research should be conducted on this topic.

\section{Boundary Fit}

Results showed that the majority of respondents preferred a segmentation level that ranged from neutral to highly segmented, whereas the majority of their workplaces ranged from neutral to highly integrated. Thus, it appears that overall, the majority of the sample suffers from misfit situations. The fact that a large number of individuals suffer from misfit confirms that this is a topic that needs more examination.

Positive boundary fit was linked consistently to increased mental health, indicating that individuals who have workplaces that fit their boundary management preferences are likely to have improved mental health. Individuals who have good fit are also less likely to have work-to-nonwork conflict, and, through that, mental health issues. Based on these conclusions, it would appear that if employers can offer their employees work-nonwork boundary supplies that match the employees' preferences, employees will have reduced conflict and increased mental health. However, contrary to hypotheses, some of the results showed that instances of good fit are detrimental to physical health.

This finding is both counterintuitive and contrary to previous research. From a systems perspective, better health in one arena should be related to better health in another arena (Bronfenbrenner, 1977). So why might the results of this dissertation be so divergent? Several potential reasons are discussed next, including 1) discrepancy 
between perceived and actual congruence, 2) potential moderators, 3) reverse causality, 4) actual differences that may or may not be a function of 5) individual differences that are a function of the non-random sample.

In order to offer potential explanations of these divergent findings, we must first take a step back to examine the construct and operationalization of "congruence". By using the Kreiner (2006) scale, this study operationalizes "congruence" as the degree to which the individual's preferences match with their perception of what their organization allows/encourages. This point is very important: this instance of operationalizing P-E fit compares an individual's preferences to their perceptions of the organizational culture. Perceptions are extremely important, and can have an influence on many things, especially mental health (i.e., the stressor-strain model posits that an individual must perceive stress before experiencing strain, e.g., Hurrell et al., 1998; Kahn \& Byosiere, 1992; Spector \& Jex, 1998). However, perceptions are not always accurate.

As mentioned in the Introduction, there are many types of fit, and many distinctions between the types of fit. One of these distinctions is the distinction between direct measures of fit and indirect measures of fit (Kristof-Brown \& Guay, 2011). A direct measure of fit is self-report of whether the individual believes fit exists. In contrast, an indirect measure of fit assesses the person and the environment components separately and calculates fit between the two. The present study examined fit in terms of indirect measures; that is, the participants were not asked whether they felt they fit with their organization, but rather, the individual's preferences for work-nonwork boundary 
management were compared to the individual's perceptions of organizational worknonwork boundary management culture or supplies.

However, within indirect measures of P-E fit, there are further distinctions between subjective fit and objective fit. Subjective fit focuses on the individual's perceptions of the "E" in P-E fit, that is, their perception of the environment. This is the technique used in the current study. The alternative, objective fit, obtains measures of the environment from other sources, such as the CEO or leader's perceptions of the organizational culture, or the average scores of all employees' assessments of the organizational culture aggregated together (Kristof-Brown \& Guay, 2011).

Both of these methods of assessing fit are valuable. Multi-source data, such as that provided in objective measures of P-E fit, is highly valued in organizational research, as it avoids the common method bias (Sonnentag \& Fritz, 2007; Sonnentag et al., 2010). However, an individual's perceived experiences don't always match up well with external data. Thus, an individual may perceive a situation of poor fit, when all objective indicators demonstrate good fit. According to P-E fit theory, the individual must perceive poor fit in order to experience bad outcomes, much in the same way the stressorstrain model works (Edwards \& Rothbard, 1999).

The preceding discussion makes it clear that both objective and subjective measures of fit have their pros and cons. Objective measures of fit give the data the robustness of multi-source data, but poor fit can occur without an individual's perception of it, and thus, without many of the attendant negative outcomes. In contrast, subjective measures of fit are subject to the common method bias, but also have the strength of 
portraying the individual's perceptions, which hold a great deal of influence over subsequent stress reactions.

In sum, future research should examine both the individual's perceptions of organizational work-nonwork culture as well as any more objective markers of the culture, such as printed and enforced company policies, managerial assessments of worknonwork boundary culture and aggregate employee assessments of overall work-nonwork boundary culture. Comparing employee preferences ("person") to both of these measures of organizational work-nonwork supplies ("environment") may yield a more complete picture of how work-nonwork boundary fit interacts with other variables. Obtaining measures from multiple sources is an excellent way to lower method bias, thus strengthening research (Podsakoff, MacKenzie, Lee, \& Podsakoff, 2003). Thus, future research should examine this construct of fit in multiple ways, from multiple sources.

The divergent mental-physical health findings may also possibly be the result of one or more moderations. As illustrated in the demographic characteristics, there was a great deal of heterogeneity in age of the sample. Thus, the relationship between boundary fit and health may look different at different points across the lifespan. Previous research has indicated that older adults respond more negatively to role stressors in terms of physical and mental health (e.g., Mayes, Barton, \& Ganster 1991), and that the relationship between role stressors and work-nonwork conflict looks different for different age groups (Matthews et al., 2010). While the current study used age as a control variable, it may be useful to examine age as a potential moderator in future research. 
The qualitative findings indicated several potential moderations that may be worth examining in future studies. For example, in the Boundary Management category, many individuals described that they found integration beneficial when it was their choice. Several explicitly stated that when integration was forced upon them, it became an unwelcome burden. This means that potentially, some individuals with poor fit feel that they have control over it (i.e., they can change it to good fit when it is convenient for them) while others with poor fit may feel powerless to alter their situations. Similarly, some individuals with good fit may feel that this is a result of their choice, while others may be aware that they do not have the flexibility to alter their boundary management strategies if they might desire change. It may be that this feeling of power, in conjunction with fit, is what influences health. Thus, future studies may find that the relationship between boundary fit and health is moderated by the amount of control an individual has over their preferred boundary management strategies.

Another potential moderation that was identified via the qualitative findings was that of position/occupation. Several individuals indicated that the organizational supplies for work-nonwork boundaries in their organization varied as a function of where the individual fell in the organization's hierarchy, such that individuals higher in the organization were unable to segment their work and nonwork lives, while hourly workers lower in the organization found it relatively easy. Thus, it may be that there is one pattern of boundary fit-health relationships for low-level employees, and another for high-level employees. Future research should be conducted in an environment were rank can be more easily discerned, such as government work, where ranks are clearly defined 
and can be quantified to determine if this is a deciding factor in how boundary fit influence health.

When conducting research in a design other than quasi-experimental or fullexperimental, the question of reverse causality must be addressed. In the current study, this is especially important, given the unexpected findings regarding mental and physical health. Is it possible that health influences boundary fit, rather than the reverse proposed in the hypotheses for this study? Yes. The current study was based on theory (i.e., boundary theory, work-family border theory, P-E fit) and previous research (e.g., Chen et al., 2009; Edwards \& Rothbard, 1999; Kreiner, 2006) and utilized two time points (i.e., boundary fit from Time 1 and Health from Time 2) in an effort to eliminate the possibility of reverse causality. However, in life, few things are strictly linear. In fact, feedback loops are often present, such as the "loss-spirals" and "gain-spirals" presented in the Conservation of Resources theory (Hobfoll, 1989). Thus, it is entirely possible that health influences perceptions of boundary fit. Consider this hypothetical situation: an employee experiences poor physical health, such as a chronic illness. This situation forces the employee to change their work-nonwork boundary management strategyperhaps they now need to integrate in order to attend doctor appointments, whereas in the past they preferred to segment. If their employer accommodates the needed shift in boundary management—say by allowing the employee to work from home some days of the week - the employee may feel that they are experiencing high boundary fit, such that their employer is flexible enough to allow them to change their boundary management strategy when needed. The knowledge that their employer is accepting, their physical 
health can be addressed, and their home life can be preserved gives the employee positive mental health. Thus, in this instance, the employee has poor physical health, which leads to perceptions of boundary fit due to an accommodating and flexible employer, which then leads to positive mental health.

However, this is merely a supposition at this point. Future research would need to be conducted with three or more time points to assess the potential of a pattern such as is described above. Additionally, like many psychological phenomena, it seems likely that these relationships may be cyclical, such that no variable is strictly a predictor or strictly an outcome, but that a much more complex picture exists. Again, future research measuring all constructs at several time points should be conducted to test this possibility.

It may be that the divergent mental-physical health findings from the current study are reflective of true patterns, at least for some workers. If this is the case, it brings some interesting conclusions regarding designing the "best" workplace for workers. As illustrated by simple averages, the majority of the sample desire work environments on the segmented end of the continuum rather than the integrated end. Thus, for the majority of this sample, instances of "good fit" would occur when an individual wants and receives segmented boundaries. In exploring the research question, it became clear that highly integrated work environments (i.e., those with low boundary supplies) were actually the best for physical health (see Figure 5 for a visual representation of this relationship). This implies that physical health would be high for individuals with low levels of boundary supplies - the opposite of what most individuals in the sample desire. Thus, it may be less a function of the effect of congruence on physical health, and more a 
function of the relative homogeneity of the sample's preferences. While most of the sample wanted to have segmented boundaries, it appears that an integrated work environment is actually better for physical health.

This means that for the majority of the sample, individuals want something that is not beneficial for their physical health. When they achieve what they desire (high segmentation preferences, high segmentation supplies), it increases their mental health. Logically, this makes sense-a sense of satisfaction is likely to occur when one achieves his or her desire. However, what they want (a highly segmented work environment) appears to be detrimental to physical health. This is similar to the concept of overindulgence in food-while having another serving of ice cream may make a person happier, it is not necessarily positive for their physical health.

Why might an integrated workplace be beneficial to physical health? The nature of integration implies that employees are expected to take time out of their work-day for personal issues (and then to subsequently take time out of their home life for work issues). It may be that this flexibility encourages employees to take time for physical exercise during their day, thus improving their physical health. It is also plausible that an integrated organization encourages employees to socialize with their coworkers outside of the workplace, rather than segmenting domains strictly between work and social life. This may mean that some employees choose to spend time with their coworkers in physical activities, such as after-work kickball leagues or basketball pick-up games. The flexibility of an integrated workplace may also allow individuals to be home for meals 
more often than those who stick to a rigid schedule, enabling them to eat more healthy home-prepared foods.

These explanations are plausible. However, why, then, would most employees prefer a segmented work environment? And why would achieving that segmentation be better for mental health? Integrated work environments require frequent role transitions, which can increase role blurring and decrease role clarity (Ashforth et al., 2000). It may well be that the increased role blurring associated with integrated workplaces is stressful and causes anxiety, thereby damaging mental health. In support of this idea, Kossek and colleagues (2006) state that individuals with high levels of role integration are subject to increased levels of cognitive complexity, which can cause frustration and distress (Kossek et al., 2006).

It is important to note that if these differences do exist, the relationship may be true only for a select group of workers. The sample utilized in this study was not random, and had several unique characteristics that make generalization of these findings to the general population unwise. For example, the participants in this sample were highly educated (over $80 \%$ had at least a four-year college degree). Similarly, their jobs were primarily white-collar jobs in the education or professional services industries. Thus, it is likely that the majority of this sample have work environments that involve a great deal of sitting, and that challenges in their workplace are primarily mental rather than physical. For this population, an integrated workplace may mean a great deal more movement. Movement is an especially critical way to improve health in the desk-bound population, as previous studies have shown that health can be severely damaged by 
sitting all day, even if the individual participates in physical activity outside of work hours (Katzmarzyk, Church, Craig, \& Bouchard, 2009). In contrast, these individuals may want a more segmented workplace so that they can focus more on their particular work environment and "get things done". Thus, it may be that this odd pattern of divergent mental and physical health outcomes is only true for individuals with specific characteristics, such as those that are highly educated, with white-collar jobs. To account for this, future research should be conducted with a random sample of individuals from a variety of types of jobs. Specifically, it may be of interest to examine blue-collar or other non-office workers and their experiences with boundary fit and health.

It is worth noting that previous studies regarding boundary fit and health found that segmentation, rather than integration, was overall more positive for health outcomes (Edwards \& Rothbard, 1999; Kossek et al., 2006). However, the findings of this current study are not necessarily counter to these previous findings, due to the affective nature of the outcomes examined in those studies. In the Edwards and Rothbard (1999) study, the outcome of "well-being" was made up of satisfaction, depression, anxiety, irritation, and somatic symptoms, while the health outcome of interest in the Kossek et al. (2006) study was depression. Thus, these results more closely map onto the current study's outcome of mental health, rather than physical health (with the exception of the somatic symptoms in the Edwards \& Rothbard 1999 article). In this sense, the current study's findings are consistent with previous findings: mental health is optimized when individuals desire and achieve segmentation, although fit overall is a more important predictor than segmentation level. The current study is the first, to my knowledge, to explicitly examine 
physical health, which is why the findings pertaining to the negative relationship between segmentation level and physical health are unique.

\section{Work-Nonwork Conflict}

Results showed that both directions of work-nonwork conflict were significantly and negatively related to mental health over time, but not physical health, partially supporting Hypothesis 1. Given that role conflict is defined as a "psychological tension", it is rational that experiencing high levels of conflict would inherently damage mental health levels (Katz \& Kahn, 1966). However, several previous studies (e.g., Grzywacz et al., 2002; Van Steenbergen \& Ellemers, 2009) have demonstrated that there is typically a link between work-nonwork conflict and physical health as well, which was not found in this study.

The lack of significant relationship between work-nonwork conflict and physical health may be due in part to the multitude of factors that influence health. Previous research has shown that a particular stressor-strain relationship, even a powerful one, can only explain about $10 \%$ of the variance in health, due to the fact that health is influenced by such a vast panoply of variables (Semmer et al., 1996).

Additionally, the three-month time lag may not have been long enough to observe physical health changes as a result of work-nonwork conflict. As mentioned previously, there is very little theoretical rationale to guide longitudinal research in the field of stress and health (Mitchell \& James, 2001). It may well be work-nonwork conflict has a more immediate effect on an individual's mental health (i.e., within three months), while the 
impact on physical health takes time to develop. Future research should examine these relationships over multiple time points in order to map the course of this relationship.

Boundary fit significantly and negatively influenced work-to-nonwork conflict, which mediated the relationship between boundary fit and health, partially supporting Hypothesis 4. As predicted in Hypothesis 4, individuals who have high levels of boundary fit have lower levels of work-to-nonwork conflict, which in turn significantly improved mental health outcomes. This implies that mental health can be protected and promoted by improving fit between an individual's boundary preferences and the boundary supplies provided by their organization.

While nonwork-to-work conflict did have an influence on mental health over time, it did not appear to be significantly influenced by the construct of boundary fit, and thus, the hypothesized mediation was not significant. The lack of a significant relationship between nonwork-to-work conflict and boundary fit may be due in part to range restriction. As evident in Table 1, the majority of the sample experienced low conflict ( 2.58 or lower on a 5.00 scale). This range restriction may have made significant differences difficult to discern. Future research should examine these relationships in a sample that has greater representation on the high conflict end of the spectrum.

Additionally, it is possible that the lack of significant findings related to nonworkto-work conflict is due to the cross-domain nature of conflict (e.g., Ford et al., 2007), such that home life "fit" would be strongly related to nonwork-to-work conflict. That is, it is understandable that the attributes of the organization are less likely to influence conflict stemming from the home domain than conflict stemming from the work domain. 
Attributes such as the amount of responsiveness expected from an individual for homedomain issues while in the workplace may be much more predictive of nonwork-to-work conflict.

\section{Work-Nonwork Enhancement}

The six types of work-nonwork enhancement did not have an overwhelmingly large impact on the outcome of health. Affective work-nonwork enhancement was positively and significantly related to physical health in both directions, but none of the other types of enhancement were significantly related to either physical or mental health. Thus, it appears that physical health can be supported by increasing affective worknonwork enhancement. Boundary fit did not seem to significantly influence any of the types of work-nonwork enhancement. As a result, there was no significant mediation as proposed in Hypothesis 5.

It seems counterintuitive that affective work-nonwork enhancement would be related to physical health rather than mental health. However, this does follow along previous lines of research. Recall that the work-nonwork enhancement scale utilized in this study was minimally adapted from the work-family positive spillover scale developed by Hanson and colleagues (2009). When conducting validation testing, the authors found that the affective sub-scale of positive spillover did not relate to outcomes as predicted, including job satisfaction, family satisfaction, and mental health (Hanson et al., 2009). Specifically, their results showed that neither direction of affective positive spillover was significantly related to mental health, as measured by the same MCS measure used in the current study (work-to-family $\mathrm{r}=-.15$, family-to-work $\mathrm{r}=.02$; 
Hanson et al., 2009). Given that the outcome scale (MCS, from the SF-12) is the same in both studies and the predictor scale is minimally different ("family" changed to "personal life"), it is understandable that the results of the present study mimic those of the original validation study. The original validation study did not include a measure of physical health, and thus, the current findings pertaining to PCS cannot be paralleled by the validation study.

Thus, it appears that the lack of significant findings linking affective worknonwork enhancement with mental health are consistent with previous research. But why might this be the case? While the MCS (and the PCS) are well-validated and reputable scales, it may be that the language regarding mental health does not parallel well between the MCS and the work-nonwork affective enhancement scale, thus creating a disjoint in the minds of participants. Looking to the individual scale language, it is apparent that the affective enhancement scale is centered around concepts of mood. For example, one item states, "When things are going well at work, my outlook regarding my personal life is improved," while another states, "Being happy at work improves my spirits at home". As is appropriate, these measures seem to relate strongly to mood, which can be fleeting. In contrast, the MCS refers to mental states such as "emotional problems (such as feeling depressed or anxious)". This language may imply to participants that mental health, as captured in the MCS, primarily encompasses clinical diagnoses such as depression or anxiety, rather than the overarching minutiae of mental health that includes things like mood, outlook, and spirits. It may be that the positive mood states illustrated by high work-nonwork affective enhancement lead to higher energy, allowing participants to 
exercise and thus feel that they have improved their physical health. However, it may take a longer time for the impacts of those cumulative mood states to improve overall more serious "emotional problems", and thus perhaps it takes longer for MCS to be influenced by affective enhancement than PCS.

The lack of significant findings regarding work-nonwork enhancement is likely the result of range restrictions (illustrated in Table 1) that consistently appeared in all enhancement scales. The majority of the respondents all experienced high levels of enhancement, consistently in the upper half of the 5-point scale. This appeared especially problematic for nonwork-to-work value-based enhancement (where the $68 \%$ of scores were 3.93 or higher) and work-to-nonwork affective enhancement (where the $68 \%$ of scores were 3.59 or higher). This range restriction may have made it impossible to detect differences within the limited range available. Future research should examine this phenomenon in a population with a wider range of enhancement scores.

An alternative explanation for the lack of significant findings regarding enhancement may be due to a true lack of relationships. Previous research has demonstrated that work-nonwork enhancement is not merely a polar opposite of worknonwork conflict, but an independent construct (e.g., Geurts \& Demerouti, 2003; Wayne, Musisca, \& Fleeson, 2004). It is true that some antecedents and outcomes overlap between the two. For example, the presence of support has been linked to both lowered levels of conflict and increased levels of enhancement (Hammer \& Zimmerman, 2011). Similarly, high levels of conflict have been related to increased levels of depression, while high levels of enhancement have been related to lower levels of depression (e.g., 
Hammer et al., 2005). However, there are other antecedents and outcomes that do not overlap between the two constructs. For example, neuroticism negatively predicts enhancement, but does not positively predict conflict (Hammer \& Zimmerman, 2011). Similarly, interventions that shorten the work week reduce conflict but do not increase enhancement; in fact, enhancement is reduced by shortening the work week (Grzywacz et al., 2002).

The fact that work-nonwork conflict and work-nonwork enhancement are separate constructs, each with their own antecedents and outcomes, may explain why boundary fit was significantly related to conflict but not to enhancement. It may be that despite the theoretical rationale presented in the introduction section of this dissertation, worknonwork boundary fit simply does not result in increased work-nonwork enhancement. Simply because good boundary fit lowers work-nonwork conflict does not mean it necessarily increases work-nonwork enhancement. Thus, the findings from this study may be another illustration of how the two constructs differ from one another.

\section{Contributions to the Literature}

While previous studies have individually studied the concepts of boundary fit, work-nonwork conflict, work-nonwork enhancement, and health, to my knowledge, no study has encompassed the relationships between all of them together. This current study strives to illustrate these constructs in one simultaneous model, which sheds light on the interrelationships between the constructs. For example, it appears that boundary management strategies that improve mental health may also damage physical health, a subtlety that would have been lost if these constructs were not all included. With this 
model, it is possible for researchers to begin to understand how the complex web of relationships between the constructs, and thus be able to make more accurate and helpful recommendations for practitioners.

The field of research encompassing boundary fit is a relatively small one. The current study contributes new pieces of knowledge to the field of boundary fit as a whole, and a more nuanced understanding of how it can influence health in complex ways. To date, very few studies have examined the impact of boundary fit on health of workers. The current study builds on the work of Edwards and Rothbard (1999) to examine not just health, but two types of health. By adding on this distinction, the current study allowed a greater depth of knowledge about how fit influences health and differential effects of fit on the two types of health.

As described earlier, boundary theory states that there is no one "right" boundary management strategy, and that positive outcomes stem instead from good fit between preferences and desires (Nippert-Eng, 1996). However, many studies examining boundary fit have also found main effects of level of segmentation as well. Very few of these studies spend a great deal of time theoretically justifying why such a finding, one that is contrary to the tenets of the theory, continues to emerge in studies focused on congruence. The findings from the current study indicate that this relationship may be inconsistent across outcome variables. Future research should pursue a deeper understanding of why segmentation supplies can sometimes have direct effects on outcomes, but not in other situations. A theoretical basis for such research would be especially beneficial, given the relative lack of purposeful exploration into this topic. 
By studying work-nonwork conflict and work-nonwork enhancement rather than the popularly used constructs of work-family conflict and work-family enhancement, the current study continues the recent movement to expand the field to populations that we know less about, namely, individuals who may be without spouses or children. And in fact, the majority of participants in this study did not have children. By using the broader work-nonwork constructs, the results of this study can more easily be generalized to nontraditional family structures and individuals without children, populations that are typically understudied in I/O psychology.

By utilizing LCM, this study provides additional support for this relatively new methodological technique. Given that the results of this study were similar to those by Edwards and Rothbard (1999) using a different method, it lends support to the idea that LCM is a viable technique for examining boundary congruence and its influence on health.

\section{Practical Applications}

Ideally, study results can point to implementation strategies should improve multiple outcomes without decreasing other desirable outcomes. The results of this dissertation did not indicate a strategy or type of fit that could adequately do such a thing. Instances of good boundary fit were beneficial for mental health but detrimental to physical health at the same time. Given these differential effects of boundary fit on the outcomes examined in this study, I would hesitate to recommend that employers encourage or emphasize a particular style of boundary management. 
With that caveat, it is worth noting that increasing boundary fit did appear to lower work-to-nonwork conflict and thereby improve mental health, both of which are desirable outcomes for individuals and organizations. Based on theory, previous research, and some of the findings in the current study, I would still recommend that organizations and employees strive to achieve good work-nonwork boundary fit. Organizations that wish to encourage work-nonwork boundary fit should begin with accurate, detailed job descriptions and provide realistic job previews for incoming candidates. The organizational culture surrounding work-nonwork boundaries should be explicitly addressed at this time. Since many aspects of organizational supplies for worknonwork boundary management are informal, rather than formal aspects, potential applications should have the opportunity to discuss the issue not only with senior leadership, but also with current workers in a casual environment. This realistic presentation of the company, especially the company's work-nonwork culture, will allow candidates with extreme cases of misfit to self-select out, limiting the amount of misfit between the individual and the organization. Specifically, organizations should be explicit and up-front about the policies, practices, and culture surrounding boundary management. This will enable applicants to make and informed decision in regards to their home life and to begin with at least a moderate amount of boundary fit, thus avoiding issues of severe misfit.

For their part, potential employees must take a moment to examine their nonwork life and determine what their ideal boundary management strategy might be. When considering a new organization, potential employees must keep this in mind, and 
proactively seek out information about the work-nonwork boundary culture of an organization. Potential employees who have the luxury of choosing an organization must take a wide variety of factors into consideration when making their choice; worknonwork boundary culture should be one of those factors. In order to feel adequately informed about the organizational work-nonwork boundary supplies, potential employees must proactively seek to understand the current culture, and the acceptance for departures from that culture.

Additionally, creating a culture of openness around the topic of boundary management may go a long way towards ameliorating tension. It is important to remember that the boundary supplies measured here were perceived boundary supplies. Some aspects of boundary supplies are relatively clear-cut, such as when an employee has a contractual obligation to serve a specific number of hours on-call during a pay period. Others, however, are much more subjective, such as the perception of when employees are expected to respond to emails. For instance, an employee may perceive that his or her work environment is highly integrated because they receive emails from managers late at night or on weekends and resent this felt obligation to integrate their own work. An open and honest conversation between coworkers, held in a no-judgment environment, may prove otherwise. It may be that while the manager prefers an integrated work environment (and thus is happy sending emails late at night or on weekends), he or she is also perfectly accepting that the staff members prefer segmentation, and would agree to not expect emailed responses on the weekend. Open discussion of boundary management styles, if endorsed and supported by upper 
management, may help employees to realize the flexible options available for them, and may increase their satisfaction.

\section{Limitations and Future Research}

The results of this study that pertained to physical health were somewhat surprising. As mentioned in the discussion of boundary fit results, it appears that integrated workplaces may be better for employee physical health, a finding that is in contrast with boundary theory's proposal that no one strategy is "best". Future research should see if this finding is replicated in other populations. If so, there is a need to investigate which aspects of the integrated workplace are especially beneficial for physical health. Is it that integrated workplaces encourage employees to leave work for nonwork activities such as exercise, thereby making it easier to fit into a busy schedule? This topic would be interesting to explore via a series of in-depth qualitative interviews with individuals who experience integrated work environments.

As mentioned previously, future research should explore potential moderators of the boundary fit-conflict-health relationship. Qualitative analyses of the open-ended responses showed that many respondents held an "it depends" attitude, a red flag for potential moderators. Participants stated that the relationship between these constructs was influenced by a variety of things, including the individual's occupation, whether boundary crossing was a choice or a requirement, and technological capabilities to enhance boundary crossing, among others. Thus, it appears that a great many factors can influence not only how boundaries are managed, but also how that makes individuals feel. 
Supporting this qualitative finding is a new theoretical model of boundary management styles, recently put forth by Kossek and Lautsch (2012). They propose that an individual's perceived control to be able to enact his or her preferred boundary management style has both direct and moderating influences on the relationship between boundary fit and work-nonwork conflict. This model was published after the current study had already been completed, and thus, could not be integrated into the design of this study. However, in another very recent study, Kossek, Ruderman, Braddy, and Hannum (2012) developed and validated a person-centered approach to examining boundary management that puts the Kossek and Lautsch (2012) theory into action.

The new approach presented by Kossek and colleagues (2012) is unique in that boundary management is examined based on a constellation of related work-nonwork factors rather than simply boundary management preferences and supplies. Their approach examined individuals' work-life boundaries via three factors: cross-role interruptions, identity centrality, and perceived control of boundaries.

Cross-role interruptions, as defined by Kossek et al. (2012) "refer to the degree to which individuals allow interruptions from one role to another." This is essentially the amount of boundary crossing that occurs for an individual. Interestingly, the scale developed and validated in Kossek et al.'s 2012 paper allows examination of fit in crossrole interruptions. Specifically, cross-role interruptions can be symmetrical (equal amounts of interruptions from work to nonwork and visa versa) or asymmetrical (allowing a greater number of interruptions in one direction than the other). 
Identity centrality as defined in the Kossek et al. (2012) paper, "reflects the identity salience and indicates the relative value the individual places on his or her different identities, which is often related to the time and energy invested in a role" (Kossek et al., 2012, Thoits, 1991). Thus, the level that one identifies with a particular role is examined, something that was not considered in the present study.

The last of the work-nonwork boundary management factors examined in Kossek et al.'s model is perceived boundary control, or the amount of control an individual believes they have over boundary crossings. This is very similar to the construct of boundary fit examined in the present study, such that individuals with high perceived boundary control or good boundary fit are able to enact their preferred boundary management strategies, while individuals with low perceived boundary control or poor boundary fit are unable to enact their preferred boundary management strategies.

By examining these three boundary management related constructs together, Kossek and colleagues (2012) were able to develop six boundary management profiles. Each of these profiles related differentially to various work-family outcomes, such as turnover, work-schedule fit, and time adequacy. Similar to the body of research surrounding boundary fit, their results showed that the two profiles that were characterized by low perceived boundary control (i.e., poor boundary fit) had worse work-family outcomes.

While the Kossek et al. (2012) was released after the current study had concluded and thus, could not influence the development of the study, it seems likely that future research examining boundary management strategies should take this person-centered 
approach into consideration, as it may describe the individual's experience better.

Accurate classification into one of the six boundary management profiles developed may lead to a better understanding of the subtleties of the work-nonwork boundary relationship, which, in turn, may lead to more accurate relationships with outcomes. Future research should utilize the person-centered approach to examine the relationship between boundary management and health, as it may provide a more accurate view of the total experience.

One limitation of the current study pertains to the method of analysis. As mentioned in the methods section, the study of congruence has been fraught with difficulties over time. Much of early congruence research was examined using difference scores and profile similarity indices, which are subject to many substantial methodological problems (Edwards, 1994). The field has since progressed to utilizing polynomial regression, the technique used here for secondary analyses (Edwards, 1994, 2002; Edwards \& Parry, 1993). The technique utilized in this dissertation for primary analyses, LCM, is a relatively new development, and has certain advantages over previous techniques (e.g., the inclusion of measurement error). However, LCM is not without drawbacks. Edwards (2009) expounds on these drawbacks, stating, "From a measurement perspective, the LCM is a step forward in congruence research, given that measurement error can bias coefficient estimates in polynomial regression and lead to erroneous conclusions. However, from a substantive perspective, the LCM is a step backward." (Edwards, 2009, pg. 35). 
At the core of Edwards' objections to LCM is the fact that LCM is by its very nature restricted to linear relationships. Thus, it cannot address curvilinear relationships, which are an important piece of congruence theory. Specifically, much of the P-E fit theory is based on the premise that outcomes decrease when the person and the environment differ in either direction (e.g., Kristof, 1996). This assumption indicates a curvilinear relationship between congruence and outcomes, one that LCM cannot capture.

Using the technique of LCM, congruence is defined as the difference between the component variables, in this case, boundary management preferences and boundary management supplies (as per Edwards' 2009 deconstruction of the Cheung 2007 equations underlying LCM). Theoretically, congruence is defined as the fit, match, or similarity between two components (Edwards, 1994). While the difference between these two definitions may seem slight, it does slightly change the interpretation of the results. Thus, for LCM, congruence is the lack of difference, while in theory, it is the presence of similarity. These definitional differences must be kept in mind when interpreting the findings of the current study, and those of other P-E fit studies that utilize LCM, such as Chen et al. (2009) and Cheung (2007).

To address these issues, Edwards and Kim (2002) propose translate polynomial regression into SEM, without using second-order constructs such as "congruence" and "level" as in LCM. At this time, there are no SEM models that I know of that can incorporate quadratic equations that will allow true congruence theories to be tested (Edwards, 2009). Future research should continue to explore methodology pertaining to congruence research. Similarly, research on the concept of congruence should always 
strive to utilize the best and most recent methods of operationalizing and measuring congruence, even if that means utilizing two types of analyses (such as LCM and polynomial regression together in a single study). Studies that examine this concept with multiple data sources and through multiple analysis techniques are likely to capitalize on the assets of each technique while counteracting the weaknesses, thus strengthening the research.

The current study was based only in self-report data, and thus, may suffer from common-method bias. Self-report does appear to be the best measure of many worknonwork constructs, as these constructs are based on individual perceptions, and thus, subject to misinterpretation from outside sources. However, future research should consider using alternative measures of health to explore these relationships. The current study used a very well respected, validated, and reliable scale to measure health (the SF12). However, when interpreting results, one must keep in mind the potential issues of construct validity. While the SF-12 does certainly measure something, and does that accurately, according to reliability estimates, that "something" may not necessarily be mental or physical health. Rather, when the individual items are examined, the SF-12 reports on symptoms of mental and physical health. Both types of health are hugely complex, and symptomology may only capture a small portion of the intricacy of the true concept. Thus, it is entirely possible that different results may appear when using measures of specific illnesses, assessments of acute vs. chronic conditions, or objective health indicators such as blood pressure, cortisol levels, or body mass index (BMI). Replicating this study with additional health outcome measures is especially important, 
given the differential and counter-intuitive results regarding mental and physical health. Additional replications with alternative outcome measures may be able to shed more light on these surprising findings, and explain this unusual pattern of outcomes. Additionally, as mentioned previously, it would be beneficial to assess congruence in terms of not only perceived organizational supplies, but also "objective" organizational supplies, as reported by others.

As discussed in the results section, some of the scales in this study suffered from range restriction issues, especially those scales pertaining to enhancement and nonworkto-work conflict. Future research should examine populations with greater variety in levels of these constructs in order to truly assess whether there are differences determined by boundary fit.

Due to the fact that the current study collected data from multiple time points, causal inferences, while still not certain, are much more robust than those in previous studies of the phenomenon (e.g., Chen et al., 2009; Edwards \& Rothbard, 1999). However, as mentioned in the results section, three time points would be an ideal design in order to assess hypotheses of mediation and have confidence in the causal directions of relationships (Shrout \& Bolger, 2002). Additionally, as some respondents noted, the time of year during which the study was conducted could influence responses, due in part to holidays, weather, or job cycles. Future research should examine these constructs at several time points in order to strengthen knowledge regarding causality and potential cyclical trends. 
Generalizability of this study is limited by the fact that the sample is not a random sample, and that it is impossible to measure the return rate of the first wave of surveys, thereby introducing potential selection bias. Future research that is larger in scope than the present study should seek to obtain a large, random sample from a diverse population where return rate on the first wave of surveys can be measured. Similarly, the population from this sample was highly educated, and many worked in the education industry. As previously discussed, this population may have a different boundary management experience than other workers. Future research examining these constructs should be conducted using low-wage workers, as they may experience these relationships differently than highly educated, well-paid individuals.

The open-ended responses also indicated that many individuals were selfemployed. The construct of boundary supplies is a difficult one for those who are selfemployed. One would assume that individuals who are self-employed get to create boundary supplies that are closely matched to their boundary preferences. However, external factors, such as the need to obtain new business, may mean that supplies do not always equal preferences. To my knowledge, no research has yet examined boundary fit in the self-employed population. The open-ended responses from this population indicate that this would be a fruitful avenue of investigation in the future.

\section{Conclusions}

The fit between an individual's work-nonwork boundary preferences and the organization's work-nonwork policies and practices has both direct and indirect effects on the health of workers. The results of the current study help us to understand the 
constructs and the relationship. Future research should continue to examine the constructs of fit between work-nonwork preferences and supplies, and how boundary management strategies influence different outcomes. 


\section{References}

Allan, C., Loudoun, R., \& Peetz, D. (2007). Influences on work/non-work conflict. Journal of Sociology, 43, 219-239.

Allen, T. D., Herst, D. E .L., Bruck, C. S., \& Sutton, M. (2000). Consequences associated with work-to-family conflict: A review and agenda for future research. Journal of Occupational Health Psychology, 5, 278-308.

Allis, P. \& O'Driscoll, M. (2008). Positive effects of nonwork-to-work facilitation on well-being, work, family, and personal domains. Journal of Managerial Psychology, 23, 273-291.

American Psychological Association (2012). Workforce Retention Survey. Results available online at http://www.apa.org/news/press/releases/phwa/index.aspx

Amstad, F.T., Meier, L.L., Fasel, U., Elfering, A., \& Semmer, N.K. (2011). A metaanalysis of work-family conflict and various outcomes with a special emphasis on cross-domain versus matching-domain relations. Journal of Occupational Health Psychology, 16, 151-169.

Aryee, S., Srinivas, E.S., \& Tan, H.H. (2005). Rhythms of life: Antecedents and outcomes of work-family balance in employed parents. Journal of Applied Psychology, 90, 132-146.

Ashforth, B.E., Kreiner, G.E., \& Fugate, M. (2000). All in a day's work: Boundaries and micro role transitions. The Academy of Management Review, 25, 472-291.

Baicker, K., Cutler, D., \& Song, Z. (2010). Workplace wellness programs can generate savings. Health Affairs, 29, 304-311. 
Barling, J., \& Frone, M.R. (2004). The Psychology of Workplace Safety. Washington, DC: American Psychological Association.

Baruch, Y., \& Holtom, B.C. (2008). Survey response rate levels and trends in organizational research. Human Relations, 61, 1139-1160.

Bennett, S.J., Oldridge, N.B., Eckert, G.J., Embree, J.L., Browning, S., Hou, N., Deer, M., \& Murray, M.D. (2002). Discriminant properties of commonly used quality of life measures in heart failure. Quality of Life Research, 11, 349-359.

Bulger, C.A., Matthews, R.A., \& Hoffman, M.E. (2007). Work and personal life boundary management: Boundary strength, work/personal life balance, and the segmentation-integration continuum. Journal of Occupational Health Psychology, 12, 365-375.

Byrne, D. (1969). Attitudes and attraction. In L. Berkowitz (Ed.), Advances in experimental social psychology (Vol. 4, pp. 35-89). New York: Academic Press.

Cable, D.M. \& Edwards, J.R. (2004). Complementary and supplementary fit: A theoretical and empirical integration. Journal of Applied Psychology, 89, 822834.

Cable, D.M. \& Judge, T.A. (1996). Person-organization fit, job choice decisions, and organizational entry. Organizational Behavior and Human Decision Processes, 67, 294-311.

Caplan, R.D. (1987). Person-environment fit theory and organizations: Commensurate dimensions, time perspectives, and mechanisms. Journal of Vocational Behavior, 31, 248-267. 
Carlson, D.S. (1999). Personality and role variables as predictors of three forms of workfamily conflict. Journal of Vocational Behavior, 55, 236-253.

Carlson, D.S., \& Kacmar, K.M. (2000). Work-family conflict in the organization: Do life role values make a difference? Journal of Management, 26, 1031-1054.

Carlson, D.S., Kacmar, K.M., Wayne, J.H., \& Grzywacz, J.G. (2006). Measuring the positive side of the work-family interface: Development and validation of a workfamily enrichment scale. Journal of Vocational Behavior, 68, 131-164.

Carlson, D.S. \& Perrewe, P.L. (1999). The role of social support in the stressor-strain relationship: An examination of work-family conflict. Journal of Management, $25,513-540$.

Carr, J. Z., Schmidt, A. M., Ford, K., \& DeShon, R. P. (2003). Climate perceptions matter: A meta-analytic path analysis relating molar climate, cognitive and affective states, and individual level work outcomes. Journal of Applied Psychology, 88, 605-619.

Carstensen, L.L. (1992). Social and emotional patterns in adulthood: Support of socioemotional selectivity theory. Psychology and Aging, 7, 331-338.

Casper, W.J., Eby, L.T., Bordeaux, C., Lockwood, A., \& Lambert, D. (2007). A review of research methods in IO/OB work-family research. Journal of Applied Psychology, 92, 28-43.

Chen, Z., Powell, G.N., Greenhaus, J.H. (2009). Work-to-family conflict, positive spillover, and boundary management: A person-environment fit approach. Journal of Vocational Behavior, 74, 82-93. 
Cheung, G.W. (2009). Introducing the latent congruence model for improving the assessment of similarity, agreement, and fit in organizational research. Organizational Research Methods, 12, 6-33.

Christie, A.M. \& Barling, J. (2009). Disentangling the indirect links between socioeconomic status and health: The dynamic roles of work stressors and personal control. Journal of Applied Psychology, 94, 1466-1478.

Clark, S.C. (2000). Work/family border theory: A new theory of work/family balance. Human Relations, 53, 747-770.

Clark, S.C. (2002). Employees' sense of community, sense of control, and work/family conflict in Native American organizations. Journal of Vocational Behavior, 61, 92-108.

Crouter, A.C. (1984). Spillover from family to work: The neglected side of the workfamily interface. Human Relations, 37, 425-441.

Danna, K., \& Griffin, R.W. (1999). Health and well-being in the workplace: A review and synthesis of the literature. Journal of Management, 25, 357-384.

Darr, W. \& Johns, G. (2008). Work strain, health, and absenteeism: A meta-analysis. Journal of Occupational Health Psychology, 13, 293-318.

Demerouti, E. (2012). The spillover and crossover of resources among partners: The role of work-self and family-self facilitation. Journal of Occupational Health Psychology, 17, 184-195.

Desrochers, S., Hilton, J.M., \& Larwood, L. (2005). Preliminary validation of the workfamily integration-blurring scale. Journal of Family Issues, 26, 442-466. 
Eby, L.T., Casper, W.J., Lockwood, A., Bordeaux, C., \& Brinley, A. (2005). Work and family research in IO/OB: Content analysis and review of the literature (19802002). Journal of Vocational Behavior, 66, 124-197.

Edington, D., \& Burton, W. (2003). Health and productivity in R. McCunney (Ed.), A Practical Approach to Occupational and Environmental Medicine (pp. 40-152). Philadelphia, PA: Lippincott Williams and Wilkins.

Edwards, J.R. (1992). A cybernetic theory of stress, coping and well-being in organizations. Academy of Management Review, 17, 161-171.

Edwards, J.R. (2002). Alternatives to difference scores: Polynomial regression analysis and response surface methodology. In F. Drasgow \& N.W. Schmitt (Eds.), Advances in measurement and data analysis (pp. 350-400). San Francisco: Jossey-Bass.

Edwards, J.R. (1991). Person-job fit: A conceptual integration, literature review, and methodological critique. In C.L. Cooper \& I.T. Robertson (Eds.), International review of industrial and organizational psychology (Vol. 6, pp. 283-357). New York: Wiley.

Edwards, J.R. (1994). The study of congruence in organizational behavior research: Critique and a proposed alternative. Organizational Behavior and Human Decision Processes, 58, 51-100.

Edwards, J.R. (1996). An examination of competing version of the person-environment fit approach to stress. Academy of Management Journal, 39, 292-339. 
Edwards, J.R., Caplan, R.D., \& Harrison, R.V. (1998). Person-environment fit theory: Conceptual foundations, empirical evidence, and directions for future research. In C.L. Cooper (Ed.), Theories of Organizational Stress (pp. 28-67). Oxford: Oxford University Press.

Edwards, J.R., \& Parry, M.E. (1993). On the use of polynomial regression equations as an alternative to difference scores in organizational research. Academy of Management Journal, 36, 1577-1613.

Edwards, J.R. \& Rothbard, N.P. (2000). Mechanisms linking work and family: Clarifying the relationship between work and family constructs. Academy of Management Review, 25, 179-199.

Edwards, J.R. \& Rothbard, N. P. (1999). Work and family stress and well-being: An examination of person-environment fit in the work and family domains. Organizational Behavior and Human Decision Processes, 77, 85-129.

Fisher, G.G., Bulger, C.A., Smith, C.S. (2009). Beyond work and family: A measure of work/nonwork interference and enhancement. Journal of Occupational Health Psychology, 14, 441-456.

Ford, M.T., Heinen, B.A., \& Langkamer, K.L. (2007). Work and family satisfaction and conflict: A meta-analysis of cross-domain relations. Journal of Applied Psychology, 92, 57-80.

Fox, M.L. \& Dwyer, D.J. (1999). An investigation of the effects of time and involvement in the relationship between stressors and work-family conflict. Journal of Occupational Health Psychology, 4, 164-174. 
French, J.R.P., Jr., Caplan, R.D., \& Harrison, R.V. (1982). The mechanisms of job stress and strain. New York: Wiley.

Frone, M.R. (2003). Work-family balance. In J. C. Quick, \& L. E. Tetrick, (Eds.), Handbook of Occupational Health Psychology. Washington, DC: American Psychological Association.

Frone, M.R. (2000). Work-family conflict and employee psychiatric disorder: The National Comorbidity Survey. Journal of Applied Psychology, 85, 888-895.

Frone, M.R., Russell, M., Cooper, M.L. (1992). Antecedents and outcomes of workfamily conflict: Testing a model of the work-family interface. Journal of Applied Psychology, 77, 65-78.

Frone, M.R., Russell, M., \& Cooper, M.L. (1997). Relation of work-family conflict to health outcomes: A four-year longitudinal study of employed parents. Journal of Occupational and Organizational Psychology, 70, 325-335.

Geurts, S.A.E., \& Demerouti, E. (2003). Work/non-work interface: A review of theories and findings. In M.J. Schabracq, J.A.M. Winnubst, \& C.L. Cooper (Eds.), The Handbook of Work and Health Psychology, Second Edition (pp. 279-312). West Sussex, England: John Wiley \& Sons Ltd.

Goetzel, R. Z., Guindon, A. M., Turshen, J.I., \& Ozminkowski, R. J. (2001). Health and productivity management: Establishing key performance measures, benchmarks, and best practices. Journal of Occupational \& Environmental Medicine, 43, 1017. 
Goetzel, R.A., Long, S.R., Ozminkowski, R.J., Hawkins, K., Wang, S., Lynch, W. (2004). Health, absence, disability, and presenteeism cost estimates of certain physical and mental health conditions affecting U.S. employers. Journal of Occupational and Environmental Medicine, 46, 398-412.

Goode, W.J. (1960). A theory of role strain. American Sociological Review, 25, 483496.

Gordon, J.R., Whelan-Berry, K.S., \& Hamilton, E.A. (2007). The relationship among work-family conflict and enhancement, organizational work-family culture, and work outcomes for older working women. Journal of Occupational Health Psychology, 12, 350-364.

Grech, M.R., Neal, A., Yeo, G., Humphreys, M., \& Smith, S. (2009). An examination of the relationship between workload and fatigue within and across consecutive days of work: Is the relationship static or dynamic? Journal of Occupational Health Psychology, 14, 231-242.

Greenhaus, J.H., Allen, T.D., \& Spector, P.E. (2006). Health consequences of work family conflict: The dark side of the work-family interface. In P.L. Perrewe and D.C. Ganster (Eds.), Research in occupational stress and well-being (Vol. 6, pp. 171-211). Amsterdam: JAI.

Greenhaus, J.H., Bedeian, A.G., \& Mossholder, K.W. (1987). Work experiences, job performance, and feelings of person and family well-being. Journal of Vocational Behavior, 31, 200-215. 
Greenhaus, J.H. \& Beutell, N.J. (1985). Sources of conflict between work and family roles. Academy of Management Review, 10, 76-88.

Greenhaus, J.H., Collins, K.M., \& Shaw, J.D. (2003). The relation between work-family balance and quality of life. Journal of Vocational Behavior, 63, 510-531.

Greenhaus, J.H., \& Powell, G.N. (2006). When work and families are allies: A theory of work-family enrichment. Academy of Management Review, 31, 72-92.

Grzywacz, J.G. (2000). Work-family spillover and health during midlife: Is managing conflict everything? American Journal of Health Promotion, 14, 236-243.

Grzywacz, J.G., Almeida, D.M., \& McDonald, D.A. (2002). Work-family spillover and daily reports of work and family stress in the adult labor force. Family Relations, 51, 28-36.

Grzywacz, J.G. \& Bass, B.L. (2003). Work, family, and mental health: Testing different models of work-family fit. Journal of Marriage and Family, 65, 248-262.

Grzywacz, J.G. \& Marks, N.F. (2000). Family, work, work-family spillover, and problem drinking during midlife. Journal of Marriage and the Family, 62, 336348.

Gutek, B.A., Searle, S., \& Klepa, L. (1991). Rational versus gender role explanations for work-family conflict. Journal of Applied Psychology, 76, 4, 560-568.

Haar, J.M., \& Bardoel, E.A. (2008). Positive spillover from the work-family interface: A study of Australian employees. Asia Pacific Journal of Human Resources, 46, 275-287. 
Hammer, L.B., Cullen, J.C., Neal, M.B, Sinclair, R.R., \& Shafiro, M.V. (2005). The longitudinal effects of work-family conflict and positive spillover on depressive symptoms among dual-earner couples. Journal of Occupational Health Psychology, 10, 138-154.

Hammer, L.B., Kossek, E.E., Yragui, N.L., Bodner, T.E., \& Hanson, G.C. (2009). Development and validation of a multidimensional measure of family supportive supervisor behaviors (FSSB). Journal of Management, 35, 837-856.

Hammer, L.B. \& Zimmerman, K. L. (2011). Quality of work life. In S. Zedeck (Ed), APA Handbook of Industrial and Organizational Psychology (Chapter, pp. ). Washington, D.C.: American Psychological Association.

Hanson, G.C., Hammer, L.B., \& Colton, C.L. (2006). Development and validation of a multidimensional scale of perceived work-family positive spillover. Journal of Occupational Health Psychology, 11,249-265.

Hardy, G.E., Woods, D., \& Wall, T.D. (2003). The impact of psychological distress on absence from work. Journal of Applied Psychology, 88, 306-314.

Hart, P.M., \& Cooper, C.L. (2001). Occupational stress: Toward a more integrated framework. In N. Anderson, D.S. Ones, H.K. Sinangil, \& C. Viswesvaran (Eds), Handbook of Industrial, Work and Organizational Psychology (vol 2: Personnel Psychology). (Chapter 5, pp. 93-114). London: Sage.

Hecht, T.D., \& Boies, K. (2009). Structure and correlates of spillover from nonwork to work: An examination of nonwork activities, well-being, and work outcomes. Journal of Occupational Health Psychology, 14, 414-426. 
Hobfoll, S.E. (1989). Conservation of resources: A new attempt at conceptualizing stress. American Psychologist, 44, 513-524.

Hockey, G.R.J. (1993). Cognitive-energetical control mechanisms in the management of work demands and psychological health. In A. Baddley \& L. Weiskrantz (Eds.), Attention, selection, awareness and control (pp. 328-345). Oxford, England: Clarendon Press.

Hurrell, J.J., Jr., Nelson, D.L., \& Simmons, B.L. (1998). Measuring job stressors and strains: Where we have been, where we are, and where we need to go. Journal of Occupational Health Psychology, 3, 368-389.

Jones, W.M., \& McKenna, J. (2002). Women and work-home conflict: A dual paradigm approach. Health Education, 102, 249-259.

Kahn, R.L., Wolfe, D.M., Quinn, R.P., Snoek, J.D., Rosenthal, R.A. (1964). Organizational stress: Studies in role conflict and ambiguity. Oxford, England: John Wiley.

Katz, D. \& Kahn, R.L. (1966). The social psychology of organizations. New York: Wiley.

Kalliath, T.J., Bluedorn, A.C., \& Strube, M.J. (1999). A test of value congruence effects. Journal of Organizational Behavior, 20, 1175-1198.

Katzmarzyk, P.T., Church, T.S., Craig, C.L., \& Bouchard, C. (2009). Sitting time and mortality from all causes, cardiovascular disease, and cancer. Medicine \& Science in Sports \& Exercise, 41, 998-1005. 
Kelloway, E.K., \& Barling, J. (2010). Leadership development as an intervention in occupational health psychology. Work \& Stress, 24, 260-279.

King, L.A., Mattimore, L.K., King, D.W., \& Adams, G.A. (1995). Family support inventory for workers: A new measure of perceived social support from family members. Journal of Organizational Behavior, 16, 235-258.

Kinnunen, U., Feldt, T., Geurts, S., \& Pulkkinen, L. (2006). Types of work-family interface: Well-being correlates of negative and positive spillover between work and family. Scandinavian Journal of Psychology, 47, 149-162.

Kirchmeyer, C. (1995). Managing the work-nonwork boundary: An assessment of organizational responses. Human Relations, 48, 515-536.

Kline, R.B. (2011). Principles and Practice of Structural Equation Modeling ( $3^{\text {rd }}$ Ed.). New York: The Guilford Press.

Kossek, E.E., \& Lautsch, B.A. (2012). Work-family boundary management styles in organizations: A cross-level model. Organizational Psychology Review, 2, 152171.

Kossek, E.E., Lautsch, B., \& Eaton, S.C. (2006). Telecommuting, control, and boundary management: Correlates of policy use and practice, job control, and work-family effectiveness. Journal of Vocational Behaviors, 68, 347-367.

Kossek, E.E. \& Ozeki, C. (1999). Bridging the work-family policy and productivity gap: a literature review. Community, Work, \& Family, 2, 7-32. 
Kossek, E.E., Ruderman, M.N., Braddy, P.W., \& Hannum, K.M. (2012). Work-nonwork boundary management profiles: A person-centered approach. Journal of Vocational Behavior, 81, 112-128.

Kreiner, G.E. (2002, August). Boundary preferences and work-family conflict: A personenvironment fit perspective. Paper presented at the annual meeting of the Academy of Management Conference, Denver, CO.

Kreiner, G.E. (2006). Consequences of work-home segmentation or integration: A person-environment fit perspective. Journal of Organizational Behavior, 27, 485507.

Kreiner, G.E., Hollensbe, E.C., \& Sheep, M.L. (2006). On the edge of identity: Boundary dynamics at the interface of individual and organizational identities. Human Relations, 59, 1315-1341.

Kristof, A.L. (1996). Person-organization fit: An integrative review of its conceptualizations, measurements, and implications. Personnel Psychology, 49, $1-49$.

Kristof-Brown, A. \& Guay, R.P. (2011). Person-environment fit. In S. Zedeck (Ed), APA Handbook of Industrial and Organizational Psychology (Chapter, pp. ). Washington, D.C.: American Psychological Association.

Landsbergis, P.A., Schnall, P.L., Pickering, T.G., Warren, K., \& Schwartz, J.E. (2003). Lower socioeconomic status among men in relation to the association between job strain and blood pressure. Scandinavian Journal of Work and Environmental Health, 29, 206-215. 
Lapierre, L.M. \& Allen, T.D. (2006). Work-supportive family, family-supportive supervision, use of organizational benefits, and problem-focused coping: Implications for work-family conflict and employee well-being. Journal of Occupational Health Psychology, 11, 169-181.

Lazarus, R.S. (1990). Theory-based stress measurement. Psychological Inquiry, 1, 3-13.

Lee, T.W., Mitchell, T.R., \& Harman, W.S. (2011). Qualitative research strategies in industrial and organizational psychology. In S. Zedeck (Ed), APA Handbook of Industrial and Organizational Psychology (Chapter, pp. ). Washington, D.C.: American Psychological Association.

Lim, S., Cortina, L.M., \& Magley, V.J. (2008). Personal and workgroup incivility: Impact on work and health outcomes. Journal of Applied Psychology, 93, 95-107.

Loehlin, J.C. (1992). Latent variable models: An introduction to factor, path, and structural analysis $\left(2^{\text {nd }}\right.$ ed.). Hillsdale, NJ: Lawrence Erlbaum.

Loeppke, R., \& Hymel, P. (2006). Good health is good business. Journal of Occupational \& Environmental Medicine, 48, 533-537.

Loeppke, R., \& Hymel, P. (2008). Health and productivity enhancement, in R. Moser, Jr. (Ed.), Effective Management of Health and Safety Programs: A practical Guide, $3^{r d} e d$. (pp. 225-243). Beverly Farm, MA: OEM Press.

Marks, S.R. (1977). Multiple roles and role strain: Some notes on human energy, time and commitment. American Sociological Review, 42, 921-966.

Marshall, C.M., Chadwick, B.A., \& Marshall, B.C. (1991). The influence of employment on family interaction, well-being, and happiness. In S.J. Bahr (Ed.) Family 
research: A sixty-year review, 1930-1990: Vol. 2 (pp. 167-229). New York: Lexington Books.

Mathieu, J.E., DeShon, R.P., \& Bergh, D.D. (2008). Mediational inferences in organizational research: Then, now, and beyond. Organizational Research Methods, 11, 203-223.

Matthews, R.A., Barnes-Farrell, J.L., \& Bulger, C.A. (2010). Advancing measurement of work and family domain boundary characteristics. Journal of Vocational Behavior, 77, 447-460.

Matthews, R.A., Bulger, C.A., \& Barnes-Farrell, J.L. (2010). Work social supports, role stressors, and work-family conflict: The moderating effect of age. Journal of Vocational Behavior, 76, 78-90.

Mayes, B.T., Barton, M.E., \& Ganster, D.C. (1991). An exploration of the moderating effect of age on job stressors-employee strain relationships. Journal of Social Behavior \& Personality, 6, 289-308.

McDowell, I. (2006). Measuring health: A guide to rating scales and questionnaires, $3^{\text {rd }}$ $E d$. New York, New York: Oxford University Press.

McNall, L.A., Nicklin, J.M., \& Masuda, A.D. (2010). A meta-analytic review of the consequences associated with work-family enrichment. Journal of Business and Psychology, 25, 381-396.

Melamed, S., Shirom, A., Toker, S., Berliner, S., \& Shapira, I. (2006). Burnout and risk of cardiovascular disease: Evidence, possible causal paths, and promising research directions. Psychological Bulletin, 132, 327-353. 
Mesmer-Magnus, J.R. \& Viswesvaran, C. (2005). Convergence between measures of work-to-family and family-to-work conflict: A meta-analytic examination. Journal of Vocational Behavior. 67, 215-232.

Mitchell, R.J., \& Bates, P. (2011). Measuring health-related productivity loss. Population Health Management, 14, 93-98.

Mitchell, T.R., \& James, L.R. (2001). Building better theory: Time and specification of when things happen. Academy of Management Review, 26, 530-547.

Muchinsky, P.M., \& Monahan, C.J. (1987). What is person-environment congruence? Supplementary versus complementary models of fit. Journal of Vocational Behavior, 31, 268-277.

Nelson, D.L., \& Simmons, B.L. (2003) Health psychology and work stress: A more positive approach. In J.C. Quick \& L.E. Tetrick (Eds.), Handbook of Occupational Health Psychology, (Chapter 5, pp.97-119). Washington, D.C.: American Psychological Association.

Netemeyer, R.C., Boles, J.S. \& McMurrian, R. (1996). Development and validation of work-family conflict and family-work conflict scales. Journal of Applied Psychology, 81, 400-410.

Nielson, T.R., Carlson, D.S., \& Lankau, M.J. (2001). The supportive mentor as a means of reducing work-family conflict. Journal of Vocational Behavior, 59, 364-381. Nippert-Eng, C.E. (1996). Home and work: Negotiating boundaries through everyday life. Chicago, IL: University of Chicago Press. 
Olson-Buchanan, J.B., \& Boswell, W.R. (2006). Blurring boundaries: Correlates of integration and segmentation between work and nonwork. Journal of Vocational Behavior, 68, 432-445.

O'Reilly, C., Chatman, J., \& Caldwell, D.F. (1991). People and organizational culture: A profile comparison approach to assessing person-organization fit. Academy of Management Journal, 34, 487-516.

Parasuraman, S. \& Greenhaus, J.H. (2002). Toward reducing some critical gaps in workfamily research. Human Resource Management Review, 12, 299-312.

Parasuraman, S., Greenhaus, J.H., \& Granrose, C.S. (1992). Role stressors, social support, and well-being among two-career couples. Journal of Organizational Behavior, 13, 339-356.

Parasuraman, S., \& Simmers, C.A. (2001). Type of employment, work-family conflict and well-being: A comparative study. Journal of Organizational Behavior, 22, $551-568$.

Park, Y., Fritz, C., \& Jex, S.M. (2011). Relationships between work-home segmentation and psychological detachment from work: The role of communication technology use at home. Journal of Occupational Health Psychology, 16, 457-467.

Perrewe, P.L., Hochwarter, W.A., \& Kiewitz, C. (1999). Value attainment: An explanation of the negative effects of work family conflict on job and life satisfaction. Journal of Occupational Health Psychology, 4, 318-326.

Perrewe, P.L., Treadway, D.C., \& Hall, A.T. (2003). The work and family interface: Conflict, family-friendly policies, and employee well-being. In D.A. Hofmann \& 
L.E. Tetrick (Eds.) Health and Safety in Organizations: A Multilevel Perspective (Chapter 10, pp. 285-315). San Francisco: Jossey-Bass.

Pietromonaco, P. R., Manis, J., \& Frohardt-Lane, K. (1986). Psychological consequences of multiple social roles. Psychology of Women Quarterly, 10, 373-382.

Ployhart, R.E., \& Vandenberg, R.J. (2010). Longitudinal research: The theory, design, and analysis of change. Journal of Management, 36, 94-120.

Podsakoff, P.M., MacKenzie, S.B., Lee, J.-Y., \& Podsakoff, N.P. (2003). Common method biases in behavioral research: A critical review of the literature and recommended remedies. Journal of Applied Psychology, 88, 879-903.

Probst, T.M. \& Estrada, A.X. (2010). Accident under-reporting among employees: Testing the moderating influence of psychological safety climate and supervisor enforcement of safety practices. Accident Analysis and Prevention, 42, 14381444.

Probst, T.M., Brubaker, T.L., \& Barsotti, A. (2008). Organizational under-reporting of injury rates: An examination of the moderating effect of organizational safety climate. Journal of Applied Psychology, 93, 1147-1154.

Raphael, D., Steinmetz, B., Renwick, R., Rootman, I., Brown, I., Sehdev, H., Phillips, S., \& Smith, T. (1999). The community quality of life project: A health promotion approach to understanding communities. Health Promotion International, 14, 197-210.

Rice, R.W., Frone, M.R., \& McFarlin, D.B. (1992). Work-nonwork conflict and the perceived quality of life. Journal of Organizational Behavior, 13, 155-168. 
Rosekind, M.R., Gregory, K.B., Mallis, M.M., Brandt, S.L., Seal, B., \& Lerner, D. (2010). The cost of poor sleep: Workplace productivity loss and associated costs. Journal of Occupational and Environmental Medicine, 52, 91-98.

Rosenman, K.D., Kalush, A., Reilly, M.J., Gardiner, J.C., Reeves, M., \& Luo, Z. (2006). How much work-related injury and illness is missed by the current national surveillance system. Journal of Occupational and Environmental Medicine, 48, 357-365.

Rothausen, T.J. (1999). 'Family' in organizational research: A review and comparison of definitions and measure. Journal of Organizational Behaviors, 20, 817-836.

Rothbard, N.P., Phillips, K.W., Dumas, T.L. (2005). Managing multiple roles: Workfamily policies and individuals' desires for segmentation. Organizational Science, 16, 243-258.

Schwartz, S.H. (1992). Universals in the context and structure of values: Theoretical advances and empirical tests in 20 countries. Advances in Experimental Social Psychology, 25, 1-65.

Semmer, N., Zapf, D., \& Greif, S. (1996). "Shared job strain”: A new approach for assessing the validity of job stress measurements. Journal of Occupational and Organizational Psychology, 69, 293-310.

Shrout, P.E., \& Bolger, N. (2002). Mediation in experimental and nonexperimental studies: New procedures and recommendations. Psychological Methods, 7, 422445. 
Smith, C.S., Folkard, S., \& Fuller, J.A. (2003). Shiftwork and working hours. In J.C. Quick \& L.E. Tetrick (Eds.), Handbook of Occupational Health Psychology (Chapter 8, pp. 163-184). Washington, DC: American Psychological Association.

Sobel, M.E. (1986). Some new results on indirect effects and their standard errors in covariance structure models. In N.B. Tuma (Ed.), Sociological methodology (pp. 159-186). San Francisco: Jossey-Bass.

Sonnentag, S. \& Fritz, C. (2007). The recovery experience questionnaire: Development and validation of a measure for assessing recuperation and unwinding from work. Journal of Occupational Health Psychology, 12, 204-221.

Sonnentag, S., Kuttler, I., \& Fritz, C. (2010). Job stressors, emotional exhaustion, and need for recovery: A multi-source study on the benefits of psychological detachment. Journal of Vocational Behavior, 76, 355-365.

Staines, G.L. (1980). Spillover versus compensation: A review of the literature on the relationship between work and nonwork. Human Relations, 33, 111-129.

Stevens, D.P., Minnottee, K.L., Mannon, S.E., \& Kiger, G. (2007). Examining the "neglected side of the work-family interface: Antecedents of positive and negative family-to-work spillover. Journal of Family Issues, 28, 242-262.

Stewart, W.F., Ricci, J.A., Chee, E., Hahn, S.R., Morganstein, D. (2003). Cost of lost productive work time among U.S. worker with depression. Journal of American Medical Association, 289, 3135-3144.

Stone-Romero, E.F. (2011). Research strategies in industrial and organizational psychology: Non-experimental, quasi-experimental, and randomized experimental 
research in special purpose and non-special purpose settings. In S. Zedeck (Ed), APA Handbook of Industrial and Organizational Psychology (Chapter, pp. ).

Washington, D.C.: American Psychological Association.

Straub, R.O. (2007). Health psychology: A biopsychosocial approach. New York: Worth Publishers.

Sui, O.-, Lu, J.-, Brough, P., Lu, C.-, Bakker, A.B., Kalliath, T., O-Driscoll, M., Phillips, D.R., Chen, W.-, Lo, D., Sit, C., \& Shi, K. (2010). Role resources and workfamily enrichment: The role of work engagement. Journal of Vocational Behavior, 77, 470-480.

Tepper, B.J., Henle, C.A., Lambert, L.S., Giacalone, R.A., \& Duffy, M.K. (2008). Abusive supervision and subordinates' organizational deviance. Journal of Applied Psychology, 93, 721-732.

Thoits, P.A. (1983). Multiple identities and psychological well-being: A reformulation and test of the social isolation hypothesis. American Sociological Review, 48, 174-187.

Thomas, L.T., \& Ganster, D.C. (1995). Impact of family-supportive work variables on work-family conflict and strain: A control perspective. Journal of Applied Psychology, 80, 6-15.

Thompson, C.A., Beauvais, L.L., \& Lyness, K.S. (1999). When work-family benefits are not enough: The influence of work-family culture on benefit utilization, organizational attachment, and work-family conflict. Journal of Vocational Behavior, 54, 392-415. 
Thompson, C.A., \& Prottas, D.J. (2006). Relationships among organizational family support, job autonomy, perceived control, and employee well-being. Journal of Occupational Health Psychology, 11, 100-118.

Tsui, A.S., \& O'Reilly, C.A. (1989). Beyond simple demographic effects: The importance of relational demography in superior subordinate dyads. Academy of Management Journal, 32, 402-423.

U.S. Department of Health and Human Services, Office of Disease Prevention and Health Promotion. Healthy People 2020. Washington, DC. Available at www.healthypeople.gov/2020. Accessed on August 3, 2011.

U.S. Department of Labor, Bureau of Labor Statistics. Workplace injuries and illnesses2010. Washington, DC: US Department of Labor; October 20, 2011. Available at www.bls.gov/news.release/pdf/osh.pdf

Van Jaarsveld, D.D., Walker, D.D., \& Skarlicki, D.P. (2010). The role of job demands and emotional exhaustion in the relationship between customer and employee incivility. Journal of Management, 36, 1486-1504.

Van Steenbergen, E. F. \& Ellemers, N. (2009). Is managing the work-family interface worthwhile? Benefits for employee health and performance. Journal of Organizational Behavior, 30(5), 617-642.

Voydanoff, P. (2002). Linkages between the work-family interface and work, family and individual outcomes: An integrative model. Journal of Family Issues, 23, 138164. 
Wallace, J.E. (1999). Work-to-nonwork conflict among married male and female lawyers. Journal of Organizational Behavior, 20, 797-816.

Ware, J.E. Jr., Kosinski, M.M., \& Keller, S.D. (1996). A 12-item short-form health survey: Construction of scales and preliminary tests of reliability and validity. Medical Care, 34, 220-233.

Ware, J.E., Jr., Kosinski, M.M., Turner-Bowker, D.M., \& Gandek, B. (2002). How to score Version 2 of the SF-12 Health Survey. Lincoln, Rhode Island: QualityMetric Inc.

Wayne, J.H., Grzywacz, J.G., Carlson, D.S., \& Kacmar, K.M. (2007). Work-family facilitation: A theoretical explanation and model of primary antecedents and consequences. Human Resources Management Review, 17, 63-76.

Wayne, J.H., Randel, A.E., \& Stevens, J. (2006). The role of identity and work-family support in work-family enrichment and its work-related consequences. Journal of Vocational Behavior, 69, 445-461.

Wilensky (1960). Segregation between work and nonwork

Williams, A., Franche, R-L., Ibrahim, S., Mustard, C.A., \& Layton, F.R. (2006). Examining the relationship between work-family spillover and sleep quality. Journal of Occupational Health Psychology, 11, 27-37.

Young, M.B. (1999). Work-family backlash: Begging the question, what's fair? Annals of the American Academy of Political and Social Science, 562, 32-46. 
Zapf, D., Dormann, C., \& Frese, M. (1996). Longitudinal studies in organizational stress research: A review of the literature with reference to methodological issues. Journal of Occupational Health Psychology, 1, 145-169.

Zerubavel, E. (1991). The fine line. New York: Free Press. 


\section{Appendices}

Appendix A: Time 1 Recruitment Materials

Appendix B: Time 1 Survey

Appendix C: Time 2 Survey

Appendix D: Time 2 Recruitment Materials

Appendix E: CFA Results

Appendix F: CFA Results (with Bootstrapping)

Appendix G: LCM Results

Appendix H: Qualitative Codebook

Appendix I: Simplified LCM without Mediator Results

Appendix J: Significance Comparisons between Time 1 and Time 2 Mediator Models Appendix K: Nonsignificant Supplementary Analyses 


\section{Appendix A: Time 1 Recruitment Materials}

Recruitment Email for Friends and Family

Subject: Invitation to Participate in the Work and Home Life Survey

Dear [name],

I'd like to invite you to be a part of an online study I'm conducting on the topic of work and home life. I'm just finishing up my doctorate in Applied Psychology at Portland State University, and the last stage is a large research project. For my research project, I'm doing an online survey about how workers balance their job and their home life. I hope to learn more about how people balance work and home responsibilities so that I can help organizations create a work environment that will make for healthier, happier workers.

I'm asking you to help me conduct this research! If you currently work 20 hours or more a week, please consider taking this online survey. It's completely confidential, all online, and will only take you about 15 minutes. Everyone who completes it will be entered in a drawing to win one of four $\$ 50$ Visa gift cards! I'll also be doing one follow-up survey that will happen about 3 months from now-it'll also be confidential, online, about 15 minutes long, and it'll give you another chance to win one of the $\$ 50$ Visa gift cards.

Whether you work 20 hours a week or not, you can help further this research by passing this email on to people you know who might be willing to take it. Please pass it on if you feel comfortable doing so-every little bit helps! The link to the survey is here: www.surveymonkey.com/personalizedlink

Please email me or give me a call if you have any questions about this survey. You can reach me on my personal email, jenna.lecomte@gmail.com, or my work email, jlecomte@pdx.edu, or by phone at 503-330-2407. You can also contact the Human Subjects Research Review Committee overseeing this study by email at $\underline{\text { hsrrc@ lists.pdx.edu or by phone at 1-877-480-4400 }}$

Thanks for reading!

Jenna LeComte-Hinely

Doctoral Candidate

Psychology Department

Portland State University 


\section{Recruitment Email for Psychology Graduate Student Association (PGSA) Listserv}

Subject: Invitation to Participate in the Work and Home Life Survey

Dear Fellow Grad Students,

I'd like to invite you to be a part of an online study I'm conducting on the topic of work and home life. I'm just finishing up my doctorate in Applied Psychology at Portland State University, and this study is a part of my dissertation. For my dissertation, I'm doing an online survey about how workers balance their job and their home life. I hope to learn more about how people balance work and home responsibilities so that I can help organizations create a work environment that will make for healthier, happier workers.

I'm asking you to help me conduct this research! If you currently work 20 hours or more a week, please consider taking this online survey. It's completely confidential, all online, and will only take you about 15 minutes. Everyone who completes it will be entered in a drawing to win one of four $\$ 50$ Visa gift cards! I'll also be doing one follow-up survey that will happen about 3 months from now-it'll also be confidential, online, about 15 minutes long, and it'll give you another chance to win one of the $\$ 50$ Visa gift cards.

Whether you work 20 hours a week or not, you can help further this research by passing this email on to people you know who might be willing to take it. Please pass it on if you feel comfortable doing so-every little bit helps! The link to the survey is here: www.surveymonkey.comlpersonalizedlink

Please email me or give me a call if you have any questions about this survey! You can reach me on my personal email, jenna.lecomte@gmail.com, or my work email, jlecomte@pdx.edu, or by phone at 503-330-2407. You can also contact the Human Subjects Research Review Committee overseeing this study by email at $\underline{\text { hsrrc@ lists.pdx.edu or by phone at 1-877-480-4400. }}$

Thanks for reading!

Jenna LeComte-Hinely

Doctoral Candidate

Psychology Department

Portland State University 


\section{Recruitment Email for the Portland Industrial Organizational Psychology Association (PIOPA) Listserv}

Subject: Invitation to Participate in the Work and Home Life Survey

\section{Dear PIOPA,}

I'd like to invite you to be a part of an online study I'm conducting on the topic of work and home life. I'm just finishing up my doctorate in Applied Psychology at Portland State University, and this study is a part of my dissertation. For my dissertation, I'm doing an online survey about how workers balance their job and their home life. I hope to learn more about how people balance work and home responsibilities so that I can help organizations create a work environment that will make for healthier, happier workers.

I'm asking you to help me conduct this research! If you currently work 20 hours or more a week, please consider taking this online survey. It's completely confidential, all online, and will only take you about 15 minutes. Everyone who completes it will be entered in a drawing to win one of four $\$ 50$ Visa gift cards! I'll also be doing one follow-up survey that will happen about 3 months from now-it'll also be confidential, online, about 15 minutes long, and it'll give you another chance to win one of the $\$ 50$ Visa gift cards.

Whether you work 20 hours a week or not, you can help further this research by passing this email on to people you know who might be willing to take it. Please pass it on if you feel comfortable doing so-every little bit helps! The link to the survey is here: WwW.surveymonkey.com\personalizedlink

Please email me or give me a call if you have any questions about this survey! You can reach me on my personal email, jenna.lecomte@gmail.com, or my work email, jlecomte@pdx.edu, or by phone at 503-330-2407. You can also contact the Human Subjects Research Review Committee overseeing this study by email at hsrrc@ lists.pdx.edu or by phone at 1-877-480-4400

Thanks for reading!

Jenna LeComte-Hinely

Doctoral Candidate

Psychology Department

Portland State University 


\section{Recruitment Posting on Social Media Homepages}

Note: This message was be posted on my own personal homepages. It was not posted on the pages of others as a personalized message.

To finish my doctorate at PSU, I'm conducting a study about work and home life and how people balance the two. I'd like to invite you to participate! The study is completely confidential, all online, and only about 15 minutes long. By completing the survey, you'll be entered to win one of four $\$ 50$ Visa gift cards. I'll be conducting a follow-up study in about 3 months that will also be confidential, online, 15 minutes long, and give you another chance to win one of the $\$ 50$ Visa gift cards. Please consider taking the survey, and/or reposting to your wall to invite others! Thank you! 


\section{Recruitment Internal Email for OMSI}

Subject: Invitation to Participate in the Work and Home Life Survey

Dear Staff,

In support of OMSI's strategic commitment to health and wellness, I invite you to participate in a study on work life, home life, and well-being.

For those of you that don't know me, my name is Jenna LeComte-Hinely, and I am a Junior Research and Evaluation Associate in our Evaluation \& Visitor Studies division. In my time away from OMSI, I am finishing my doctorate at Portland State University in Applied Psychology. As a part of this program, I am conducting a large-scale online survey and I'd like OMSI to be a part of it. The study is about how workers balance their job and their home life and how this relates to their health and wellness. I hope to learn more about how people balance work and home responsibilities so that I can help organizations like OMSI create a work environment that will make for healthier, happier workers.

I invite you to participate in this study. If you currently work 20 hours or more a week for pay, please consider taking this online survey. It's completely confidential, all online, and will take you only about 15 minutes. Everyone who completes it will be entered in a drawing to win one of four $\$ 50$ Visa gift cards! I'll also be doing one follow-up survey that will happen about three months from now-it'll also be confidential, online, about 15 minutes long, and it'll give you another chance to win one of the $\$ 50$ Visa gift cards.

Whether you work 20 hours a week for pay or not, you can help further this research by passing this e-mail on to people you know who might be willing to take it. Please pass it on if you feel comfortable doing so-every little bit helps! The link to the survey is here: https://www.surveymonkey.com/s/workandhomelife

I will be sharing my overall research results with OMSI staff in a few months. I hope that my findings will help OMSI better understand how to help all of us balance work and home lives in order to continue in our efforts to make OMSI a great place to work.

Please e-mail me or give me a call if you have any questions about this survey. You can reach me by e-mail here at OMSI (jlecomtehinely@omsi.edu) or at PSU (jlecomte@pdx.edu) or by phone at 503.330.2407. You can also contact the Human Subjects Research Review Committee overseeing this study by e-mail at

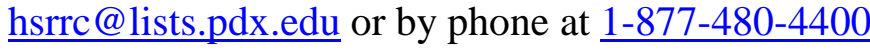

Thanks for reading!

Jenna LeComte-Hinely, MS 
Doctoral Candidate

Psychology Department

Portland State University

\section{Reminder}

To: \#AllStaff

From: jlecomtehinely@omsi.edu

Subject: Reminder: Invitation to Participate in the Work and Home Life Survey

Dear Staff,

If you are interested in participating in the Work and Home Life Survey (see below), please be sure to complete the online survey soon! Thank you!

Jenna LeComte-Hinely, MS

Doctoral Candidate

Psychology Department

Portland State University

(copied text of original e-mail here) 


\section{[Page 1: Informed Consent]}

\section{Appendix B: Time 1 Survey}

Thank you for your interest in participating in the Work and Home Life Survey! You are invited to participate in a research study conducted by Jenna LeComte-Hinely from Portland State University's psychology department. Jenna hopes to learn a few things about how you balance your work and home life. This study is being conducted in partial fulfillment of Jenna's doctoral degree, and is under the supervision of her advisor, Dr. Leslie Hammer. This study is intended for individuals who work outside the home at least 20 hours a week or more. You were selected as a possible participant in this study because you are currently employed.

If you decide to participate, you'll be asked to complete an online survey-one now and one again in about three months. Both of these surveys should take you about 15 minutes or less to complete, and will ask you questions about how you balance work and home life, and about your health. We don't anticipate that this survey will cause you any harm or discomfort. In fact, this survey may give you a feeling of satisfaction in knowing that your responses are helping to build knowledge that can help workers in the future.

All information collected during this study will be kept confidential. That means electronic data be password protected, and only trained investigators will have access to the data. No one else - not your coworkers, not your boss, not your organization-will see your individual responses to this survey.

We will not ask your name, but we will ask you to share your email address. We will only use your email address to send you an invitation for a follow-up survey approximately three months from now (the second survey will be the last survey we ask you to do-promise!) and, if you complete both surveys, to enter you in a drawing to win one of four $\$ 50$ Visa gift cards. We promise to never share or distribute your email address; you will not receive any spam as a result of sharing your email address with us. Your email address will not be connected to your survey answers in any way, so you can be comfortable knowing that your responses will be analyzed in a completely anonymous method.

Your participation is completely voluntary-you do not have to take part in this study, and it will not impact your relationship with Jenna, Portland State, or your employer. You can skip any questions that make you uncomfortable, or quit at any time.

If you have any concerns or problems about your participation in this study, or your rights as a research subject, please contact the Human Subjects Research Review Committee, Research and Strategic Partnerships, Market Center Building Suite 620, 1600 SW 4th Ave, Portland OR 97207, (503) 725-4288 / 1-877-480-4400. If you have questions about the study itself, please contact Jenna LeComte-Hinely at jlecomte@pdx.edu or 503-330-2407.

If this all sounds OK to you, please click "Next" to proceed to the survey! 
[Page 2: Exclusion Criteria]

1. Do you currently work 20 hours a week or more?

- Yes

- No

If 'yes' continue on to rest of survey. If 'no', continue on to a page with the following text:

Thank you for your willingness to participate in our survey! However, at this time we need information from people who spend a significant number of hours working outside the home.

You can still help this research, though, by passing the survey link on to individuals you know who work 20 hours per week or more and who might be willing to take this survey.

If you have any questions or concerns, please contact the primary researcher or the Human Subjects Research Review Committee overseeing this project.

Primary researcher: Jenna LeComte-Hinely

Phone: 503-330-2407

Email: jlecomte@pdx.edu

Human Subjects Research Review Committee

Phone: 1-877-480-4400

Email: hsrrc@lists.pdx.edu

Thanks again! 


\section{[Page 3: Predictors]}

1. The following statements are about your preference for managing both work and home life. Please select the rating that best describes your preferences.

\begin{tabular}{|l|l|l|l|l|l|l|l|}
\hline & $\begin{array}{l}1 \\
\text { Strongly } \\
\text { Disagree }\end{array}$ & 2 & 3 & 4 & 5 & 6 & 7 \\
\hline $\begin{array}{l}\text { I don't like to have to think about work } \\
\text { while I'm at home }\end{array}$ & & & & & & \\
\hline I prefer to keep work life at work & & & & & & & \\
\hline $\begin{array}{l}\text { I don't like work issues creeping into my } \\
\text { home life }\end{array}$ & & & & & & \\
\hline $\begin{array}{l}\text { I like to be able to leave work behind } \\
\text { when I go home }\end{array}$ & & & & & \\
\hline
\end{tabular}

2. Please select the rating that best describes how you perceive your workplace.

\begin{tabular}{|l|l|l|l|l|l|l|l|}
\hline & $\begin{array}{l}1 \\
\text { Strongly } \\
\text { Disagree }\end{array}$ & 2 & 3 & $\begin{array}{l}4 \\
\text { Neutral }\end{array}$ & 5 & 6 & $\begin{array}{l}7 \\
\text { Strongly } \\
\text { agree }\end{array}$ \\
\hline $\begin{array}{l}\text { My workplace lets people forget about } \\
\text { work when they're at home }\end{array}$ & & & & & \\
\hline $\begin{array}{l}\text { Where I work, people can keep work } \\
\text { matters at work }\end{array}$ & & & & & \\
\hline $\begin{array}{l}\text { At my workplace, people are able to } \\
\text { prevent work issues from creeping into } \\
\text { their home life }\end{array}$ & & & & & & \\
\hline $\begin{array}{l}\text { Where I work, people can mentally leave } \\
\text { work behind when they go home }\end{array}$ & & & & & \\
\hline
\end{tabular}

3. Is there anything else about this topic that you think we should know in order to fully understand your work and home life?

[open-ended text box] 


\section{[Page 4: Work-Nonwork Conflict Measure]}

1. In the past month, how often have you felt...

\begin{tabular}{|l|l|l|l|l|l|}
\hline & $\begin{array}{l}\text { Not } \\
\text { at all }\end{array}$ & $\begin{array}{l}2 \\
\text { Rarely }\end{array}$ & $\begin{array}{l}3 \\
\text { Some } \\
\text { times }\end{array}$ & $\begin{array}{l}4 \\
\text { Often }\end{array}$ & $\begin{array}{l}5 \\
\text { Almost } \\
\text { all the } \\
\text { time }\end{array}$ \\
\hline $\begin{array}{l}\text { I come home from work too tired to do the } \\
\text { things I would like to do }\end{array}$ & & & & & \\
\hline $\begin{array}{l}\text { My job makes it difficult to maintain the kind } \\
\text { of personal life I would like }\end{array}$ & & & & & \\
\hline $\begin{array}{l}\text { I often neglect my personal needs because of } \\
\text { the demands of my work }\end{array}$ & & & & & \\
\hline My personal life suffers because of my work & & & & & \\
\hline $\begin{array}{l}\text { I have to miss out on important personal } \\
\text { activities due to the amount of time I spend } \\
\text { doing work }\end{array}$ & & & & & \\
\hline
\end{tabular}

2. In the past month, how often have you felt...

\begin{tabular}{|l|l|l|l|l|l|}
\hline & $\begin{array}{l}\text { Not } \\
\text { at all }\end{array}$ & $\begin{array}{l}2 \\
\text { Rarely }\end{array}$ & $\begin{array}{l}3 \\
\text { Some } \\
\text { times }\end{array}$ & $\begin{array}{l}4 \\
\text { Often }\end{array}$ & $\begin{array}{l}5 \\
\text { Almost } \\
\text { all the } \\
\text { time }\end{array}$ \\
\hline $\begin{array}{l}\text { My personal life drains me of the energy I need } \\
\text { to do my job }\end{array}$ & & & & \\
\hline $\begin{array}{l}\text { My work suffers because of everything going } \\
\text { on in my personal life }\end{array}$ & & & & & \\
\hline $\begin{array}{l}\text { I would devote more time to work if it weren't } \\
\text { for everything I have going on in my personal } \\
\text { life }\end{array}$ & & & & & \\
\hline $\begin{array}{l}\text { I am too tired to be effective at work because of } \\
\text { things I have going on in my personal life }\end{array}$ & & & & & \\
\hline $\begin{array}{l}\text { When I'm at work, I worry about things I need } \\
\text { to do outside of work }\end{array}$ & & & & & \\
\hline $\begin{array}{l}\text { I have difficulty getting my work done because } \\
\text { I am preoccupied with personal matters at work }\end{array}$ & & & & & \\
\hline
\end{tabular}

3. Is there anything else about this topic that you think we should know in order to fully understand your work and home life?

[open-ended text box] 


\section{[Page 5: Work-Nonwork Enhancement Measures]}

1. Please rate your level of agreement with the following statements:

\begin{tabular}{|l|l|l|l|l|l|}
\hline & $\begin{array}{l}\text { Strongly } \\
\text { disagree } \\
1\end{array}$ & $\begin{array}{l}\text { Agree } \\
2\end{array}$ & $\begin{array}{l}\text { Neutral } \\
3\end{array}$ & $\begin{array}{l}\text { Agree } \\
4\end{array}$ & $\begin{array}{l}\text { Strongly } \\
\text { Agree } \\
5\end{array}$ \\
\hline $\begin{array}{l}\text { Skills developed at work help me in my } \\
\text { personal life }\end{array}$ & & & & & \\
\hline $\begin{array}{l}\text { Successfully performing tasks at work } \\
\text { helps me to more effectively accomplish } \\
\text { personal tasks }\end{array}$ & & & & & \\
\hline $\begin{array}{l}\text { Behaviors required by my job lead to } \\
\text { behaviors that assist me in my personal } \\
\text { life }\end{array}$ & & & & & \\
\hline $\begin{array}{l}\text { Carrying out my personal responsibilities } \\
\text { is made easier by using behaviors } \\
\text { performed at work }\end{array}$ & & & & & \\
\hline
\end{tabular}

2. Please rate your level of agreement with the following statements:

\begin{tabular}{|l|l|l|l|l|l|}
\hline & $\begin{array}{l}\text { Strongly } \\
\text { disagree } \\
1\end{array}$ & $\begin{array}{l}\text { Agree } \\
2\end{array}$ & $\begin{array}{l}\text { Neutral } \\
3\end{array}$ & $\begin{array}{l}\text { Agree } \\
4\end{array}$ & $\begin{array}{l}\text { Strongly } \\
\text { Agree } \\
5\end{array}$ \\
\hline $\begin{array}{l}\text { Values developed at work make me a } \\
\text { better person outside of work. }\end{array}$ & & & & & \\
\hline $\begin{array}{l}\text { I apply my workplace values in nonwork } \\
\text { situations }\end{array}$ & & & & & \\
\hline $\begin{array}{l}\text { Values I learn through my work } \\
\text { experiences assist me in fulfilling my } \\
\text { personal responsibilities }\end{array}$ & & & & & \\
\hline
\end{tabular}

3. Please rate your level of agreement with the following statements:

\begin{tabular}{|l|l|l|l|l|l|}
\hline & $\begin{array}{l}\text { Strongly } \\
\text { disagree } \\
1\end{array}$ & $\begin{array}{l}\text { Agree } \\
2\end{array}$ & $\begin{array}{l}\text { Neutral } \\
3\end{array}$ & $\begin{array}{l}\text { Agree } \\
4\end{array}$ & $\begin{array}{l}\text { Strongly } \\
\text { Agree } \\
5\end{array}$ \\
\hline $\begin{array}{l}\text { When things are going well at work, my } \\
\text { outlook regarding my personal life is } \\
\text { improved }\end{array}$ & & & & & \\
\hline $\begin{array}{l}\text { Being in a positive mood at work helps } \\
\text { me to be in a positive mood at home }\end{array}$ & & & & & \\
\hline $\begin{array}{l}\text { Being happy at work improves my spirits } \\
\text { at home }\end{array}$ & & & & & \\
\hline $\begin{array}{l}\text { Having a good day at work allows me to } \\
\text { be optimistic with my family }\end{array}$ & & & & & \\
\hline
\end{tabular}


4. Please rate your level of agreement with the following statements:

\begin{tabular}{|l|l|l|l|l|l|}
\hline & $\begin{array}{l}\text { Strongly } \\
\text { disagree } \\
1\end{array}$ & $\begin{array}{l}\text { Disagree } \\
2\end{array}$ & $\begin{array}{l}\text { Neutral } \\
\text { Skills developed in my personal life } \\
\text { help me in my job }\end{array}$ & $\begin{array}{l}\text { Agree } \\
4\end{array}$ & $\begin{array}{l}\text { Strongly } \\
\text { Agree } \\
5\end{array}$ \\
\hline $\begin{array}{l}\text { Successfully performing tasks in my } \\
\text { personal life helps me to more } \\
\text { effectively accomplish tasks at work }\end{array}$ & & & & \\
\hline $\begin{array}{l}\text { Behaviors required in my personal life } \\
\text { lead to behaviors that assist me at } \\
\text { work. }\end{array}$ & & & & & \\
\hline $\begin{array}{l}\text { Carrying out my work responsibilities } \\
\text { is made easier by using behaviors } \\
\text { performed as a part of my personal } \\
\text { life. }\end{array}$ & & & & & \\
\hline
\end{tabular}

5. Please rate your level of agreement with the following statements:

\begin{tabular}{|l|l|l|l|l|l|}
\hline & $\begin{array}{l}\text { Strongly } \\
\text { disagree } \\
1\end{array}$ & $\begin{array}{l}\text { Disagree } \\
2\end{array}$ & $\begin{array}{l}\text { Neutral } \\
3\end{array}$ & $\begin{array}{l}\text { Agree } \\
4\end{array}$ & $\begin{array}{l}\text { Strongly } \\
\text { Agree } \\
5\end{array}$ \\
\hline $\begin{array}{l}\text { My personal values make me a better } \\
\text { employee }\end{array}$ & & & & & \\
\hline $\begin{array}{l}\text { I apply my personal values in work } \\
\text { situations }\end{array}$ & & & & & \\
\hline $\begin{array}{l}\text { My personal values assist me in } \\
\text { fulfilling my work responsibilities }\end{array}$ & & & & \\
\hline
\end{tabular}

6. Please rate your level of agreement with the following statements:

\begin{tabular}{|l|l|l|l|l|l|}
\hline & $\begin{array}{l}\text { Strongly } \\
\text { disagree } \\
1\end{array}$ & $\begin{array}{l}\text { Disagree } \\
2\end{array}$ & $\begin{array}{l}\text { Neutral } \\
3\end{array}$ & $\begin{array}{l}\text { Agree } \\
4\end{array}$ & $\begin{array}{l}\text { Strongly } \\
\text { Agree } \\
5\end{array}$ \\
\hline $\begin{array}{l}\text { When things are going well in my } \\
\text { home life, my outlook regarding my } \\
\text { job is improved }\end{array}$ & & & & & \\
\hline $\begin{array}{l}\text { Being in a positive mood at home } \\
\text { helps me to be in a positive mood at } \\
\text { work }\end{array}$ & & & & & \\
\hline $\begin{array}{l}\text { Being happy at home improves my } \\
\text { spirits at work }\end{array}$ & & & & & \\
\hline $\begin{array}{l}\text { Having a good day outside of work } \\
\text { allows me to be optimistic at work }\end{array}$ & & & & & \\
\hline
\end{tabular}


3. Is there anything else about this topic that you think we should know in order to fully understand your work and home life?

[open-ended text box] 


\section{[Page 6: Outcome Measures]}

1. In general, would you say your health is:

\begin{tabular}{|l|l|l|l|l|}
\hline Excellent & Very Good & Good & Fair & Poor \\
\hline
\end{tabular}

2. The following questions are about activities you might do during a typical day. Does your health now limit you in these activities? If so, how much?

\begin{tabular}{|l|l|l|l|}
\hline & $\begin{array}{l}\text { Yes, } \\
\text { limited } \\
\text { a lot }\end{array}$ & $\begin{array}{l}\text { Yes, } \\
\text { limited a } \\
\text { little }\end{array}$ & $\begin{array}{l}\text { No, not } \\
\text { limited } \\
\text { at all }\end{array}$ \\
\hline $\begin{array}{l}\text { Moderate activities, such as moving a table, pushing a } \\
\text { vacuum cleaner, bowling, or playing golf }\end{array}$ & & & \\
\hline Climbing several flights of stairs & & & \\
\hline
\end{tabular}

3. During the past month, how much of the time have you had any of the following problems with your work or other regular daily activities as a result of your physical health?

\begin{tabular}{|l|l|l|l|l|l|}
\hline & $\begin{array}{l}\text { All of } \\
\text { the } \\
\text { time }\end{array}$ & $\begin{array}{l}\text { Most } \\
\text { of the } \\
\text { time }\end{array}$ & $\begin{array}{l}\text { Some } \\
\text { of the } \\
\text { time }\end{array}$ & $\begin{array}{l}\text { A } \\
\text { little } \\
\text { of the } \\
\text { time }\end{array}$ & $\begin{array}{l}\text { None } \\
\text { of the } \\
\text { time }\end{array}$ \\
\hline Accomplished less than you would like & & & & & \\
\hline $\begin{array}{l}\text { Were limited in the kind of work or other } \\
\text { activities }\end{array}$ & & & & & \\
\hline
\end{tabular}

4. During the past month, how much of the time have you had any of the following problems with your work or other regular daily activities as a result of any emotional problems (such as feeling depressed or anxious)?

\begin{tabular}{|l|l|l|l|l|l|}
\hline & $\begin{array}{l}\text { All of } \\
\text { the } \\
\text { time }\end{array}$ & $\begin{array}{l}\text { Most } \\
\text { of the } \\
\text { time }\end{array}$ & $\begin{array}{l}\text { Some } \\
\text { of the } \\
\text { time }\end{array}$ & $\begin{array}{l}\text { A } \\
\text { little } \\
\text { of the } \\
\text { time }\end{array}$ & $\begin{array}{l}\text { None } \\
\text { of the } \\
\text { time }\end{array}$ \\
\hline Accomplished less than you would like & & & & & \\
\hline $\begin{array}{l}\text { Did work or other activities less carefully } \\
\text { than usual }\end{array}$ & & & & & \\
\hline
\end{tabular}

5. During the past month, how much did pain interfere with your normal work (including both work outside the home and housework)?

\begin{tabular}{|l|l|l|l|l|}
\hline Not at all & A little bit & Moderately & Quite a bit & Extremely \\
\hline
\end{tabular}


6. These questions are about how you feel and how things have been with you during the past month. For each question, please give the one answer that comes closest to the way you have been feeling. How much of the time during the past four weeks...

\begin{tabular}{|l|l|l|l|l|l|}
\hline & $\begin{array}{l}\text { All of } \\
\text { the } \\
\text { time }\end{array}$ & $\begin{array}{l}\text { Most } \\
\text { of the } \\
\text { time }\end{array}$ & $\begin{array}{l}\text { Some } \\
\text { of the } \\
\text { time }\end{array}$ & $\begin{array}{l}\text { A little } \\
\text { of the } \\
\text { time }\end{array}$ & $\begin{array}{l}\text { None } \\
\text { of the } \\
\text { time }\end{array}$ \\
\hline Have you felt calm and peaceful? & & & & & \\
\hline Did you have a lot of energy? & & & & & \\
\hline Have you felt downhearted and depressed? & & & & & \\
\hline
\end{tabular}

7. During the past month, how much of the time has your physical health or emotional problems interfered with your social activities (like visiting friends, relatives, etc.)?

\begin{tabular}{|l|l|l|l|l|}
\hline All of the time & $\begin{array}{l}\text { Most of the } \\
\text { time }\end{array}$ & $\begin{array}{l}\text { Some of the } \\
\text { time }\end{array}$ & $\begin{array}{l}\text { A little of the } \\
\text { time }\end{array}$ & $\begin{array}{l}\text { None of the } \\
\text { time }\end{array}$ \\
\hline
\end{tabular}

8. Is there anything else that you think we should know in order to fully understand your health?

[open-ended text box] 


\section{[Page 7: Additional Measures]}

Please tell us about your leisure time during the past month.

1. During off-work time in the past few weeks...

\begin{tabular}{|l|l|l|l|l|l|}
\hline & $\begin{array}{l}\text { Not at } \\
\text { all }\end{array}$ & Rarely & $\begin{array}{l}\text { Some of } \\
\text { the time }\end{array}$ & $\begin{array}{l}\text { Most of } \\
\text { the time }\end{array}$ & Always \\
\hline I forget about work & & & & & \\
\hline I don't think about work at all & & & & & \\
\hline I distance myself from my work & & & & & \\
\hline $\begin{array}{l}\text { I get a break from the demands of } \\
\text { work }\end{array}$ & & & & & \\
\hline
\end{tabular}

2. Is there anything else about your leisure time you think we should know?

\section{[open-ended text box]}

3. Please tell us about how you feel about your organization's values regarding work and home life balance:

\begin{tabular}{|c|c|c|c|c|c|}
\hline & $\begin{array}{l}\text { Not at } \\
\text { all } \\
1\end{array}$ & 2 & 3 & 4 & $\begin{array}{l}\text { Completely } \\
5\end{array}$ \\
\hline $\begin{array}{l}\text { To what degree do you feel your values regarding } \\
\text { work and home life "match" or fit this organization } \\
\text { and the current employees in this organization? }\end{array}$ & & & & & \\
\hline $\begin{array}{l}\text { My values surrounding work and home life match } \\
\text { those of the current employees in the organization }\end{array}$ & & & & & \\
\hline $\begin{array}{l}\text { Do you think the values about work and home life } \\
\text { and the "personality" of this organization reflect your } \\
\text { own work and home life values and personality? }\end{array}$ & & & & & \\
\hline
\end{tabular}

4. Is there anything else about your organization's values that you think we should know?

[open-ended text box] 


\section{[Page 8: Control Measures]}

Now we'd like to ask you some questions about yourself.

1. How did you hear about this survey?
a. Email from Jenna LeComte-Hinely
b. Email from someone else
c. Internal company email
d. PIOPA email list
e. Facebook post from Jenna LeComte-Hinely
f. Facebook post from someone else
g. Google + post from Jenna LeComte-Hinely
h. Google + post from someone else
i. About.com Psychology Forum
j. About.com Working Mom Forum
k. Other:

2. What is your gender?
a. Male
b. Female

3. What is your age?

4. What is your household status?

a. Living with a spouse or partner

b. Have spouse or partner, not living together

c. Not living with a spouse or partner

5. How many children under the age of 18 live with you in your home?

6. How many children over the age of 18 live with you in your home?

7. What industry do you work in? [drop down menu]

a. Agriculture, forestry, fishing and hunting

b. Mining

c. Utilities

d. Construction

e. Manufacturing

f. Wholesale Trade

g. Retail Trade

h. Transportation and Warehousing

i. Information, Finance, and Insurance

j. Real Estate, Rental, and Leasing

k. Professional, Scientific, and Technical Services

1. Management of Companies and Enterprises

m. Administrative and Support

n. Waste Management and Remediation Services

o. Education Services

p. Health Care and Social Assistance

q. Arts, Entertainment, and Recreation 
r. Accommodation and Foodservices

s. Other Services

t. Public Administration

8. What is your job type?

9. How many hours do you work per week on an average week?

10. What is the highest level of education you've obtained?

a. Grade school or less

b. Some high school

c. High school diploma or equivalency

d. Some college or other higher education, no diploma or certification

e. Trade school completed

f. 2 year college degree

g. 4 year college degree

h. Graduate degree

11. What is your approximate household income per year, before taxes?

a. Less than $\$ 20,000$

b. $\$ 21,000$ to $\$ 40,000$

c. $\$ 41,000$ to $\$ 60,000$

d. $\$ 61,000$ to $\$ 80,000$

e. $\$ 81,000$ to $\$ 100,000$

f. $\$ 100,000$ or above

12. Is there anything you'd like to add about these questions or this survey in general?

a. [open ended text box] 
[Page 9: E-mail]

Please enter your email address in the box below. This email address will only be used 1) to enter you to win one of the four $\$ 50$ Visa gift cards, and 2) to send you an invitation to complete a second survey in approximately three months (this will be the last survey we ask you to complete).

We promise we will NOT sell or distribute your email in any way. The only individuals who will see your email are researchers who have been highly trained to protect the privacy of participants like you. Your email will never be used to identify your survey responses. All email addresses will be removed from the data before analysis so that $\underline{\text { no }}$ one will connect your answers to you.

Email: [text box for email]

Confirm email: [text box for email] 
[Page 10: Thank you]

Thank you so much for completing the Work and Home Life Survey! Your participation helps us to better understand how to make workers healthy and happy at work and outside of it.

If you entered your email on the last page, you have been entered in a drawing to win one of four $\$ 50$ Visa gift cards. We will be notifying the winners sometime during August, so keep a lookout for the email in case you are a winner!

In order to get a good sense of how people's work and home lives change over time, we'll be conducting a second survey in approximately three months. We will send you an email at that time to invite you to participate once more. It will be the last survey (promise!) and will also be short, confidential, and about work and home and how you manage the two. Completing this survey will give you another chance to win one of the $\$ 50$ Visa gift cards!

If you haven't already, please consider passing this survey link to other people you know who work 20 hours a week or more and might be willing to take this survey. The more people who take the survey, the better we can understand how balancing work and home responsibilities works for all workers.

If you would like to receive a copy of the results of this study, please send an email indicating your desire for an e-pamphlet of results to the primary researcher, Jenna LeComte-Hinely, at jlecomte@ @dx.edu. We estimate that the results will be available in December of 2012, and we would be happy to share our findings with interested individuals!

If you have any questions or concerns about this survey, you can contact the primary researcher, Jenna LeComte-Hinely, at jlecomte@ @dx.edu or 503-330-2407. You can also contact the Human Subjects Research Review Committee overseeing this study by email at hsrrc@lists.pdx.edu or by phone at 1-877-480-4400. 


\section{Appendix C: Time 2 Survey}

\section{[Page 1: Informed Consent]}

You are invited to participate in a research study conducted by Jenna LeComte-Hinely from Portland State University's psychology department. Jenna hopes to learn a few things about how you balance your work and home life. This study is being conducted in partial fulfillment of Jenna's doctoral degree, and is under the supervision of her advisor, Dr. Leslie Hammer. This study is intended as a follow up to the Work and Home Life Survey administered about three months ago. You were selected as a possible participant in this study because you participated in the Work and Home Life Survey approximately three months ago.

If you decide to participate, you'll be asked to complete one last online survey. This survey is very similar to the last one you took-it should take you about 15 minutes or less to complete, and will ask you questions about how you balance work and home life, and about your health. We don't anticipate that this survey will cause you any harm or discomfort. In fact, this survey may give you a feeling of satisfaction in knowing that your responses are helping to build knowledge that can help workers in the future.

All information collected during this study will be kept confidential. That means electronic data be password protected, and only trained investigators will have access to the data. No one else-not your coworkers, not your boss, not your organization-will see your individual responses to this survey.

We will not ask your name, but we will ask you to share your email address. We will only use your email address connect your two surveys together, and to enter you in a drawing to win one of four $\$ 50$ Visa gift cards. We promise to never share or distribute your email address; you will not receive any spam as a result of sharing your email address with us. Your email address will not be connected to your survey answers in any way, so you can be comfortable knowing that your responses will be analyzed in a completely anonymous method.

Your participation is completely voluntary-you do not have to take part in this study, and it will not impact your relationship with Jenna, Portland State, or your employer. You can skip any questions that make you uncomfortable, or quit at any time.

If you have any concerns or problems about your participation in this study, or your rights as a research subject, please contact the Human Subjects Research Review Committee, Research and Strategic Partnerships, Market Center Building Suite 620, 1600 SW 4th Ave, Portland OR 97207, (503) 725-4288 / 1-877-480-4400. If you have questions about the study itself, please contact Jenna LeComte-Hinely at jlecomte@pdx.edu or 503-330-2407.

If this all sounds OK to you, please click "Next" to proceed to the survey! Thank you! 


\section{[Page 2: Predictors]}

1. The following statements are about your preference for managing both work and home life. Please select the rating that best describes your preferences.

\begin{tabular}{|l|l|l|l|l|l|l|l|}
\hline & $\begin{array}{l}1 \\
\text { Strongly } \\
\text { Disagree }\end{array}$ & 2 & 3 & $\begin{array}{l}4 \\
\text { Neutral }\end{array}$ & 5 & 6 & $\begin{array}{l}7 \\
\text { Strongly } \\
\text { Agree }\end{array}$ \\
\hline $\begin{array}{l}\text { I don't like to have to think about work } \\
\text { while I'm at home }\end{array}$ & & & & & & \\
\hline I prefer to keep work life at work & & & & & & \\
\hline $\begin{array}{l}\text { I don't like work issues creeping into my } \\
\text { home life }\end{array}$ & & & & & \\
\hline $\begin{array}{l}\text { I like to be able to leave work behind } \\
\text { when I go home }\end{array}$ & & & & & \\
\hline
\end{tabular}

2. Please select the rating that best describes how you perceive your workplace.

\begin{tabular}{|l|l|l|l|l|l|l|l|}
\hline & $\begin{array}{l}\text { Strongly } \\
\text { Disagree }\end{array}$ & 2 & 3 & $\begin{array}{l}4 \\
\text { Neutral }\end{array}$ & 5 & 6 & $\begin{array}{l}7 \\
\text { Strongly } \\
\text { agree }\end{array}$ \\
\hline $\begin{array}{l}\text { My workplace lets people forget about } \\
\text { work when they' re at home }\end{array}$ & & & & & & \\
\hline $\begin{array}{l}\text { Where I work, people can keep work } \\
\text { matters at work }\end{array}$ & & & & & & \\
\hline $\begin{array}{l}\text { At my workplace, people are able to } \\
\text { prevent work issues from creeping into } \\
\text { their home life }\end{array}$ & & & & & \\
\hline $\begin{array}{l}\text { Where I work, people can mentally leave } \\
\text { work behind when they go home }\end{array}$ & & & & & & \\
\hline
\end{tabular}

3. Is there anything else about this topic that you think we should know in order to fully understand your work and home life?

[open-ended text box] 


\section{[Page 3: Work-Nonwork Conflict Measure]}

1. In the past month, how often have you felt...

\begin{tabular}{|l|l|l|l|l|l|}
\hline & $\begin{array}{l}1 \\
\text { Not } \\
\text { at all }\end{array}$ & $\begin{array}{l}2 \\
\text { Rarely }\end{array}$ & $\begin{array}{l}3 \\
\text { Some } \\
\text { times }\end{array}$ & $\begin{array}{l}4 \\
\text { Often }\end{array}$ & $\begin{array}{l}5 \\
\text { Almost } \\
\text { all the } \\
\text { time }\end{array}$ \\
\hline $\begin{array}{l}\text { I come home from work too tired to do the } \\
\text { things I would like to do }\end{array}$ & & & & & \\
\hline $\begin{array}{l}\text { My job makes it difficult to maintain the kind } \\
\text { of personal life I would like }\end{array}$ & & & & & \\
\hline $\begin{array}{l}\text { I often neglect my personal needs because of } \\
\text { the demands of my work }\end{array}$ & & & & & \\
\hline My personal life suffers because of my work & & & & & \\
\hline $\begin{array}{l}\text { I have to miss out on important personal } \\
\text { activities due to the amount of time I spend } \\
\text { doing work }\end{array}$ & & & & & \\
\hline
\end{tabular}

2. In the past month, how often have you felt...

\begin{tabular}{|l|l|l|l|l|l|}
\hline & $\begin{array}{l}\text { Not } \\
\text { at all }\end{array}$ & $\begin{array}{l}2 \\
\text { Rarely }\end{array}$ & $\begin{array}{l}3 \\
\text { Some } \\
\text { times }\end{array}$ & $\begin{array}{l}4 \\
\text { Often }\end{array}$ & $\begin{array}{l}5 \\
\text { Almost } \\
\text { all the } \\
\text { time }\end{array}$ \\
\hline $\begin{array}{l}\text { My personal life drains me of the energy I need } \\
\text { to do my job }\end{array}$ & & & & \\
\hline $\begin{array}{l}\text { My work suffers because of everything going } \\
\text { on in my personal life }\end{array}$ & & & & & \\
\hline $\begin{array}{l}\text { I would devote more time to work if it weren't } \\
\text { for everything I have going on in my personal } \\
\text { life }\end{array}$ & & & & & \\
\hline $\begin{array}{l}\text { I am too tired to be effective at work because of } \\
\text { things I have going on in my personal life }\end{array}$ & & & & & \\
\hline $\begin{array}{l}\text { When I'm at work, I worry about things I need } \\
\text { to do outside of work }\end{array}$ & & & & & \\
\hline $\begin{array}{l}\text { I have difficulty getting my work done because } \\
\text { I am preoccupied with personal matters at work }\end{array}$ & & & & & \\
\hline
\end{tabular}

3. Is there anything else about this topic that you think we should know in order to fully understand your work and home life?

[open-ended text box] 


\section{[Page 4: Work-Nonwork Enhancement Measures]}

1. Please rate your level of agreement with the following statements:

\begin{tabular}{|l|l|l|l|l|l|}
\hline & $\begin{array}{l}\text { Strongly } \\
\text { disagree } \\
1\end{array}$ & $\begin{array}{l}\text { Agree } \\
2\end{array}$ & $\begin{array}{l}\text { Neutral } \\
3\end{array}$ & $\begin{array}{l}\text { Agree } \\
4\end{array}$ & $\begin{array}{l}\text { Strongly } \\
\text { Agree } \\
5\end{array}$ \\
\hline $\begin{array}{l}\text { Skills developed at work help me in my } \\
\text { personal life }\end{array}$ & & & & & \\
\hline $\begin{array}{l}\text { Successfully performing tasks at work } \\
\text { helps me to more effectively accomplish } \\
\text { personal tasks }\end{array}$ & & & & & \\
\hline $\begin{array}{l}\text { Behaviors required by my job lead to } \\
\text { behaviors that assist me in my personal } \\
\text { life }\end{array}$ & & & & & \\
\hline $\begin{array}{l}\text { Carrying out my personal responsibilities } \\
\text { is made easier by using behaviors } \\
\text { performed at work }\end{array}$ & & & & & \\
\hline
\end{tabular}

2. Please rate your level of agreement with the following statements:

\begin{tabular}{|l|l|l|l|l|l|}
\hline & $\begin{array}{l}\text { Strongly } \\
\text { disagree } \\
1\end{array}$ & $\begin{array}{l}\text { Agree } \\
2\end{array}$ & $\begin{array}{l}\text { Neutral } \\
3\end{array}$ & $\begin{array}{l}\text { Agree } \\
4\end{array}$ & $\begin{array}{l}\text { Strongly } \\
\text { Agree } \\
5\end{array}$ \\
\hline $\begin{array}{l}\text { Values developed at work make me a } \\
\text { better person outside of work. }\end{array}$ & & & & & \\
\hline $\begin{array}{l}\text { I apply my workplace values in nonwork } \\
\text { situations }\end{array}$ & & & & & \\
\hline $\begin{array}{l}\text { Values I learn through my work } \\
\text { experiences assist me in fulfilling my } \\
\text { personal responsibilities }\end{array}$ & & & & & \\
\hline
\end{tabular}

3. Please rate your level of agreement with the following statements:

\begin{tabular}{|l|l|l|l|l|l|}
\hline & $\begin{array}{l}\text { Strongly } \\
\text { disagree } \\
1\end{array}$ & $\begin{array}{l}\text { Agree } \\
2\end{array}$ & $\begin{array}{l}\text { Neutral } \\
3\end{array}$ & $\begin{array}{l}\text { Agree } \\
4\end{array}$ & $\begin{array}{l}\text { Strongly } \\
\text { Agree } \\
5\end{array}$ \\
\hline $\begin{array}{l}\text { When things are going well at work, my } \\
\text { outlook regarding my personal life is } \\
\text { improved }\end{array}$ & & & & & \\
\hline $\begin{array}{l}\text { Being in a positive mood at work helps } \\
\text { me to be in a positive mood at home }\end{array}$ & & & & & \\
\hline $\begin{array}{l}\text { Being happy at work improves my spirits } \\
\text { at home }\end{array}$ & & & & & \\
\hline $\begin{array}{l}\text { Having a good day at work allows me to } \\
\text { be optimistic with my family }\end{array}$ & & & & & \\
\hline
\end{tabular}


4. Please rate your level of agreement with the following statements:

\begin{tabular}{|l|l|l|l|l|l|}
\hline & $\begin{array}{l}\text { Strongly } \\
\text { disagree } \\
1\end{array}$ & $\begin{array}{l}\text { Disagree } \\
2\end{array}$ & $\begin{array}{l}\text { Neutral } \\
\text { Skills developed in my personal life } \\
\text { help me in my job }\end{array}$ & $\begin{array}{l}\text { Agree } \\
4\end{array}$ & $\begin{array}{l}\text { Strongly } \\
\text { Agree } \\
5\end{array}$ \\
\hline $\begin{array}{l}\text { Successfully performing tasks in my } \\
\text { personal life helps me to more } \\
\text { effectively accomplish tasks at work }\end{array}$ & & & & \\
\hline $\begin{array}{l}\text { Behaviors required in my personal life } \\
\text { lead to behaviors that assist me at } \\
\text { work. }\end{array}$ & & & & & \\
\hline $\begin{array}{l}\text { Carrying out my work responsibilities } \\
\text { is made easier by using behaviors } \\
\text { performed as a part of my personal } \\
\text { life. }\end{array}$ & & & & & \\
\hline
\end{tabular}

5. Please rate your level of agreement with the following statements:

\begin{tabular}{|l|l|l|l|l|l|}
\hline & $\begin{array}{l}\text { Strongly } \\
\text { disagree } \\
1\end{array}$ & $\begin{array}{l}\text { Disagree } \\
2\end{array}$ & $\begin{array}{l}\text { Neutral } \\
3\end{array}$ & $\begin{array}{l}\text { Agree } \\
4\end{array}$ & $\begin{array}{l}\text { Strongly } \\
\text { Agree } \\
5\end{array}$ \\
\hline $\begin{array}{l}\text { My personal values make me a better } \\
\text { employee }\end{array}$ & & & & & \\
\hline $\begin{array}{l}\text { I apply my personal values in work } \\
\text { situations }\end{array}$ & & & & & \\
\hline $\begin{array}{l}\text { My personal values assist me in } \\
\text { fulfilling my work responsibilities }\end{array}$ & & & & \\
\hline
\end{tabular}

6. Please rate your level of agreement with the following statements:

\begin{tabular}{|l|l|l|l|l|l|}
\hline & $\begin{array}{l}\text { Strongly } \\
\text { disagree } \\
1\end{array}$ & $\begin{array}{l}\text { Disagree } \\
2\end{array}$ & $\begin{array}{l}\text { Neutral } \\
3\end{array}$ & $\begin{array}{l}\text { Agree } \\
4\end{array}$ & $\begin{array}{l}\text { Strongly } \\
\text { Agree } \\
5\end{array}$ \\
\hline $\begin{array}{l}\text { When things are going well in my } \\
\text { home life, my outlook regarding my } \\
\text { job is improved }\end{array}$ & & & & & \\
\hline $\begin{array}{l}\text { Being in a positive mood at home } \\
\text { helps me to be in a positive mood at } \\
\text { work }\end{array}$ & & & & & \\
\hline $\begin{array}{l}\text { Being happy at home improves my } \\
\text { spirits at work }\end{array}$ & & & & & \\
\hline $\begin{array}{l}\text { Having a good day outside of work } \\
\text { allows me to be optimistic at work }\end{array}$ & & & & & \\
\hline
\end{tabular}


3. Is there anything else about this topic that you think we should know in order to fully understand your work and home life?

[open-ended text box] 


\section{[Page 5: Outcome Measures]}

1. In general, would you say your health is:

\begin{tabular}{|l|l|l|l|l|}
\hline Excellent & Very Good & Good & Fair & Poor \\
\hline
\end{tabular}

2. The following questions are about activities you might do during a typical day. Does your health now limit you in these activities? If so, how much?

\begin{tabular}{|l|l|l|l|}
\hline & $\begin{array}{l}\text { Yes, } \\
\text { limited } \\
\text { a lot }\end{array}$ & $\begin{array}{l}\text { Yes, } \\
\text { limited a } \\
\text { little }\end{array}$ & $\begin{array}{l}\text { No, not } \\
\text { limited } \\
\text { at all }\end{array}$ \\
\hline $\begin{array}{l}\text { Moderate activities, such as moving a table, pushing a } \\
\text { vacuum cleaner, bowling, or playing golf }\end{array}$ & & & \\
\hline Climbing several flights of stairs & & & \\
\hline
\end{tabular}

3. During the past month, how much of the time have you had any of the following problems with your work or other regular daily activities as a result of your physical health?

\begin{tabular}{|l|l|l|l|l|l|}
\hline & $\begin{array}{l}\text { All of } \\
\text { the } \\
\text { time }\end{array}$ & $\begin{array}{l}\text { Most } \\
\text { of the } \\
\text { time }\end{array}$ & $\begin{array}{l}\text { Some } \\
\text { of the } \\
\text { time }\end{array}$ & $\begin{array}{l}\text { A } \\
\text { little } \\
\text { of the } \\
\text { time }\end{array}$ & $\begin{array}{l}\text { None } \\
\text { of the } \\
\text { time }\end{array}$ \\
\hline Accomplished less than you would like & & & & & \\
\hline $\begin{array}{l}\text { Were limited in the kind of work or other } \\
\text { activities }\end{array}$ & & & & & \\
\hline
\end{tabular}

4. During the past month, how much of the time have you had any of the following problems with your work or other regular daily activities as a result of any emotional problems (such as feeling depressed or anxious)?

\begin{tabular}{|l|l|l|l|l|l|}
\hline & $\begin{array}{l}\text { All of } \\
\text { the } \\
\text { time }\end{array}$ & $\begin{array}{l}\text { Most } \\
\text { of the } \\
\text { time }\end{array}$ & $\begin{array}{l}\text { Some } \\
\text { of the } \\
\text { time }\end{array}$ & $\begin{array}{l}\text { A } \\
\text { little } \\
\text { of the } \\
\text { time }\end{array}$ & $\begin{array}{l}\text { None } \\
\text { of the } \\
\text { time }\end{array}$ \\
\hline Accomplished less than you would like & & & & & \\
\hline $\begin{array}{l}\text { Did work or other activities less carefully } \\
\text { than usual }\end{array}$ & & & & & \\
\hline
\end{tabular}

5. During the past month, how much did pain interfere with your normal work (including both work outside the home and housework)?

\begin{tabular}{|l|l|l|l|l|}
\hline Not at all & A little bit & Moderately & Quite a bit & Extremely \\
\hline
\end{tabular}


6. These questions are about how you feel and how things have been with you during the past month. For each question, please give the one answer that comes closest to the way you have been feeling. How much of the time during the past month...

\begin{tabular}{|l|l|l|l|l|l|}
\hline & $\begin{array}{l}\text { All of } \\
\text { the } \\
\text { time }\end{array}$ & $\begin{array}{l}\text { Most } \\
\text { of the } \\
\text { time }\end{array}$ & $\begin{array}{l}\text { Some } \\
\text { of the } \\
\text { time }\end{array}$ & $\begin{array}{l}\text { A little } \\
\text { of the } \\
\text { time }\end{array}$ & $\begin{array}{l}\text { None } \\
\text { of the } \\
\text { time }\end{array}$ \\
\hline Have you felt calm and peaceful? & & & & & \\
\hline Did you have a lot of energy? & & & & & \\
\hline Have you felt downhearted and depressed? & & & & & \\
\hline
\end{tabular}

7. During the past month, how much of the time has your physical health or emotional problems interfered with your social activities (like visiting friends, relatives, etc.)?

\begin{tabular}{|l|l|l|l|l|}
\hline All of the time & $\begin{array}{l}\text { Most of the } \\
\text { time }\end{array}$ & $\begin{array}{l}\text { Some of the } \\
\text { time }\end{array}$ & $\begin{array}{l}\text { A little of the } \\
\text { time }\end{array}$ & $\begin{array}{l}\text { None of the } \\
\text { time }\end{array}$ \\
\hline
\end{tabular}

8. Is there anything else that you think we should know in order to fully understand your health?

[open-ended text box] 


\section{[Page 6: Other Measures]}

Please tell us about your leisure time during the past month.

1. During off-work time in the past few weeks...

\begin{tabular}{|l|l|l|l|l|l|}
\hline & $\begin{array}{l}\text { Not at } \\
\text { all }\end{array}$ & Rarely & $\begin{array}{l}\text { Some of } \\
\text { the time }\end{array}$ & $\begin{array}{l}\text { Most of } \\
\text { the time }\end{array}$ & Always \\
\hline I forget about work & & & & & \\
\hline I don't think about work at all & & & & & \\
\hline I distance myself from my work & & & & & \\
\hline $\begin{array}{l}\text { I get a break from the demands of } \\
\text { work }\end{array}$ & & & & & \\
\hline
\end{tabular}

2. Is there anything else about your leisure time you think we should know?

[open-ended text box]

3. Please tell us about how you feel about your organization's values regarding work and home life balance:

\begin{tabular}{|c|c|c|c|c|c|}
\hline & $\begin{array}{l}\text { Not at } \\
\text { all } \\
1\end{array}$ & 2 & 3 & 4 & $\begin{array}{l}\text { Completely } \\
5\end{array}$ \\
\hline $\begin{array}{l}\text { To what degree do you feel your values regarding } \\
\text { work and home life "match" or fit this organization } \\
\text { and the current employees in this organization? }\end{array}$ & & & & & \\
\hline $\begin{array}{l}\text { My values surrounding work and home life match } \\
\text { those of the current employees in the organization }\end{array}$ & & & & & \\
\hline $\begin{array}{l}\text { Do you think the values about work and home life } \\
\text { and the "personality" of this organization reflect your } \\
\text { own work and home life values and personality? }\end{array}$ & & & & & \\
\hline
\end{tabular}

4. Is there anything else about your organization's values that you think we should know?

[open-ended text box] 


\section{[Page 7: Control Measures]}

Now we'd like to ask you some questions about yourself.

1. What is your gender?
a. Male
b. Female
c. Transgender

2. What is your age?

3. What is your household status?

a. Living with a spouse or partner

b. Only adult in the household (alone or with children)

c. Living with adult roommates or other adult family members

4. How many children under the age of 18 live with you in your home?

5. How many children over the age of 18 live with you in your home?

6. What industry do you work in? [drop down menu]

a. Agriculture, forestry, fishing and hunting

b. Mining

c. Utilities

d. Construction

e. Manufacturing

f. Wholesale Trade

g. Retail Trade

h. Transportation and Warehousing

i. Information, Finance, and Insurance

j. Real Estate, Rental, and Leasing

k. Professional, Scientific, and Technical Services

1. Management of Companies and Enterprises

m. Administrative and Support

n. Waste Management and Remediation Services

o. Education Services

p. Health Care and Social Assistance

q. Arts, Entertainment, and Recreation

r. Accommodation and Foodservices

s. Other Services

t. Public Administration

7. What is your job type?

8. How many hours do you work per week on an average week?

9. What is the highest level of education you've obtained?
a. Grade school or less
b. Some high school
c. High school diploma or equivalency
d. Some college or other higher education, no diploma or certification
e. Trade school completed 
f. 2 year college degree

g. 4 year college degree

h. Graduate degree

10. What is your approximate household income per year, before taxes?

a. Less than $\$ 10,000$

b. Between $\$ 10,000$ and $\$ 14,999$

c. Between $\$ 15,000$ and $\$ 24,999$

d. Between $\$ 25,000$ and $\$ 34,999$

e. Between $\$ 35,000$ and $\$ 49,999$

f. Between $\$ 50,000$ and $\$ 74,999$

g. Between $\$ 75,000$ and $\$ 99,000$

h. Between $\$ 100,000$ and $\$ 149,999$

i. Between $\$ 150,000$ and $\$ 199,999$

j. $\$ 200,000$ or more

11. Is there anything you'd like to add about these questions or this survey in general?

a. [open ended text box] 
[Page 8: E-mail]

Please enter your email address in the box below. This email address will only be used to enter you in a drawing to win one of four $\$ 50$ Visa gift cards.

We promise we will NOT sell or distribute your email in any way. The only individuals who will see your email are researchers who have been highly trained to protect the privacy of participants like you. Your email will never be used to identify your survey. All email addresses will be removed from the data before analysis so that no one will connect your answers to you.

Email: [text box for email]

Confirm email: [text box for email] 


\section{[Page 9: Thank you]}

Thank you so much for completing the follow-up to the Work and Home Life Survey! Your participation helps us to better understand how to make workers healthy and happy at work and outside of it.

This is the last survey we will ask you to do. If you entered your email on the last page, we'll be doing the drawing for one of four $\$ 50$ Visa gift cards around August, so watch your email to see if you are one of the winners!

If you would like to receive a copy of the results of this study, please send an email indicating your desire for an e-pamphlet of results to the primary researcher, Jenna LeComte-Hinely, at jlecomte@pdx.edu. We estimate that the results will be available in December of 2012, and we would be happy to share our findings with interested individuals! If you've already contacted the researcher to indicate your interest in receiving a copy of the results, there's no need to sign up a second time.

If you have any questions or concerns, you can contact the primary researcher, Jenna LeComte-Hinely, at jlecomte@pdx.edu or 503-330-2407. You can also contact the Human Subjects Research Review Committee overseeing this study by email at hsrrc@ lists.pdx.edu or by phone at 1-877-480-4400. 


\section{Initial Email}

\section{Appendix D: Time 2 Recruitment Materials}

Subject: Invitation to Participate in the Final Work and Home Life Survey

Greetings!

The researchers at Portland State University would like to thank you again for completing the Work and Home Life Survey that you took online in [month]. Your responses are helping us to understand how workers manage to balance both home and work

responsibilities, and to get a better grasp on what organizations can do to make that easier for workers. Because you participated in that survey, you have been entered in a drawing to win one of four $\$ 50$ gift cards.

In order to get a complete picture of how people balance work and home responsibilities, it's important that we survey people multiple times. This allows us to look at how things change over time, and gives us a better idea of how to design workplaces that can foster healthy, happy employees. In order to get this type of data, we're conducting a second, follow-up survey, and we'd like to ask you to participate.

This second survey is also about work and home life and how you balance the two. It will be completely confidential, and should only take about 15 minutes to complete. If you complete this second survey, you'll have another chance to win-you'll be entered a second time in the drawing to win one of four $\$ 50$ Visa gift cards!

The link to the survey is here: www.surveymonkey.com\personalized

It would be a big help for our research if you could complete this second survey by [date].

If you have any questions about this survey or the previous one, please don't hesitate to contact the primary researcher, Jenna LeComte-Hinely, via email at jlecomte@ @dx.edu or via phone at 503-330-2407.

Thanks for contributing your time to this study, we greatly appreciate your responses!

Jenna LeComte-Hinely, MS

Doctoral Student

Psychology Department

Portland State University 


\section{Reminder Email \#1}

Subject: Reminder: Invitation to Participate in the Final Work and Home Life Survey

Good [morning, afternoon, evening, dependent on time]!

Thanks again for taking the first Work and Home Life Survey in [month]! Your responses are extremely helpful for our study, and we're learning a great deal from them. As a token of our appreciation, your email has been entered in a drawing to win one of four $\$ 50$ Visa gift cards.

However, to complete our study, we'd really like to hear from you again so we can understand how you balance work and home life over time and what changes might have happened. Please consider taking the follow-up to the Work and Home Life Survey! The link to the survey is here: www.surveymonkey.comlpersonalizedlink

This will be the last survey in our study. Just like the last survey, it should take you about 15 minutes to complete, and it'll be totally confidential. It's also about work and home life and how you balance them. If you complete it, you'll get another chance to win one of the $\$ 50$ Visa gift cards-your email will be entered in the drawing a second time if you complete this second survey!

If you have any questions or concerns, please contact the primary researcher, Jenna LeComte-Hinely, at jlecomte@pdx.edu or by phone at 503-330-2407. You can also contact the Human Subjects Research Review Committee overseeing this study by email at hsrrc@ @ists.pdx.edu or by phone at 1-877-480-4400.

Thank you for your time!

--Jenna LeComte-Hinely 


\section{Reminder Email \#2}

Subject: Survey Ending Soon! Please Share Your Experience!

Hello again,

Please consider filling out the follow-up to the Work and Home Life Survey. It's all online, about 15 minutes long, and totally confidential. Your response will help us to understand how work and home life interact for workers everywhere, and what we can do to make that experience better. If you complete it, you'll get another chance to win one of four $\$ 50$ Visa gift cards.

The link to the survey is here: [SurveyLink]

If you have any questions or concerns, please contact the primary researcher, Jenna LeComte-Hinely, at jlecomte@ pdx.edu or by phone at 503-330-2407. You can also contact the Human Subjects Research Review Committee overseeing this study by email at hsrrc@lists.pdx.edu or by phone at 1-877-480-4400.

Thank you again for your participation in the first survey. We hope to hear from you soon!

Sincerely,

Jenna LeComte-Hinely, M.S.

Doctoral Candidate

Psychology Department

Portland State University 


\section{Appendix E: CFAs}

\section{Boundary Preferences}

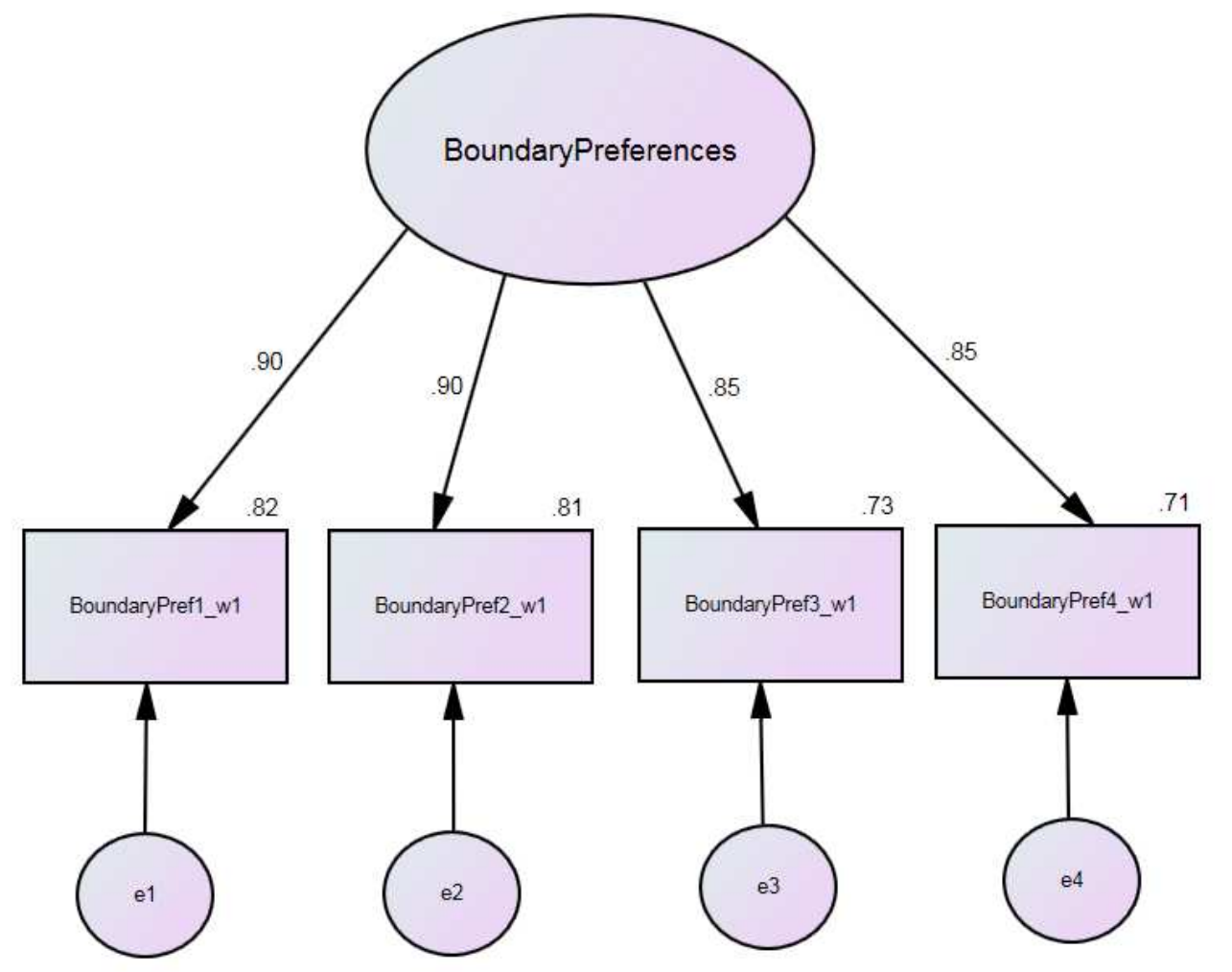

Fit Statistics:

- $\chi^{2}(2)=11.500, p>.001$

- $\mathrm{CFI}=.990$

- $\mathrm{GFI}=.980$

- RMSEA = .128

- Highest absolute value of the standardized residual covariances $=.533$

Conclusion: Adequate fit 


\section{Boundary Supplies}

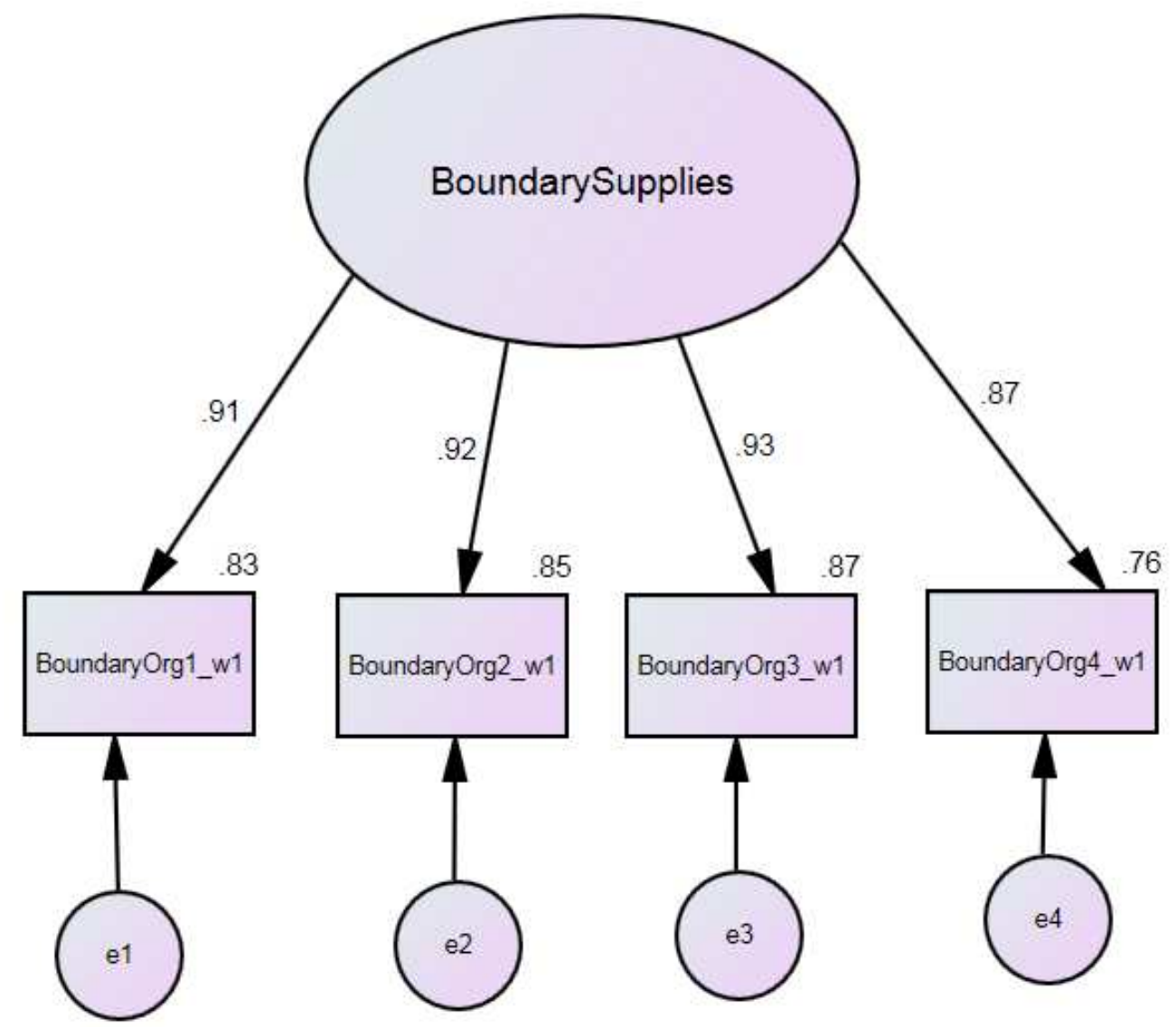

Fit Statistics:

- $\chi^{2}(2)=9.127, p>.001$

- $\mathrm{CFI}=.994$

- $\mathrm{GFI}=.985$

- RMSEA = .111

- Highest absolute value of the standardized residual covariances $=.255$

Conclusion: Adequate fit 


\section{Work-to-Nonwork Behavior-Based Enhancement}

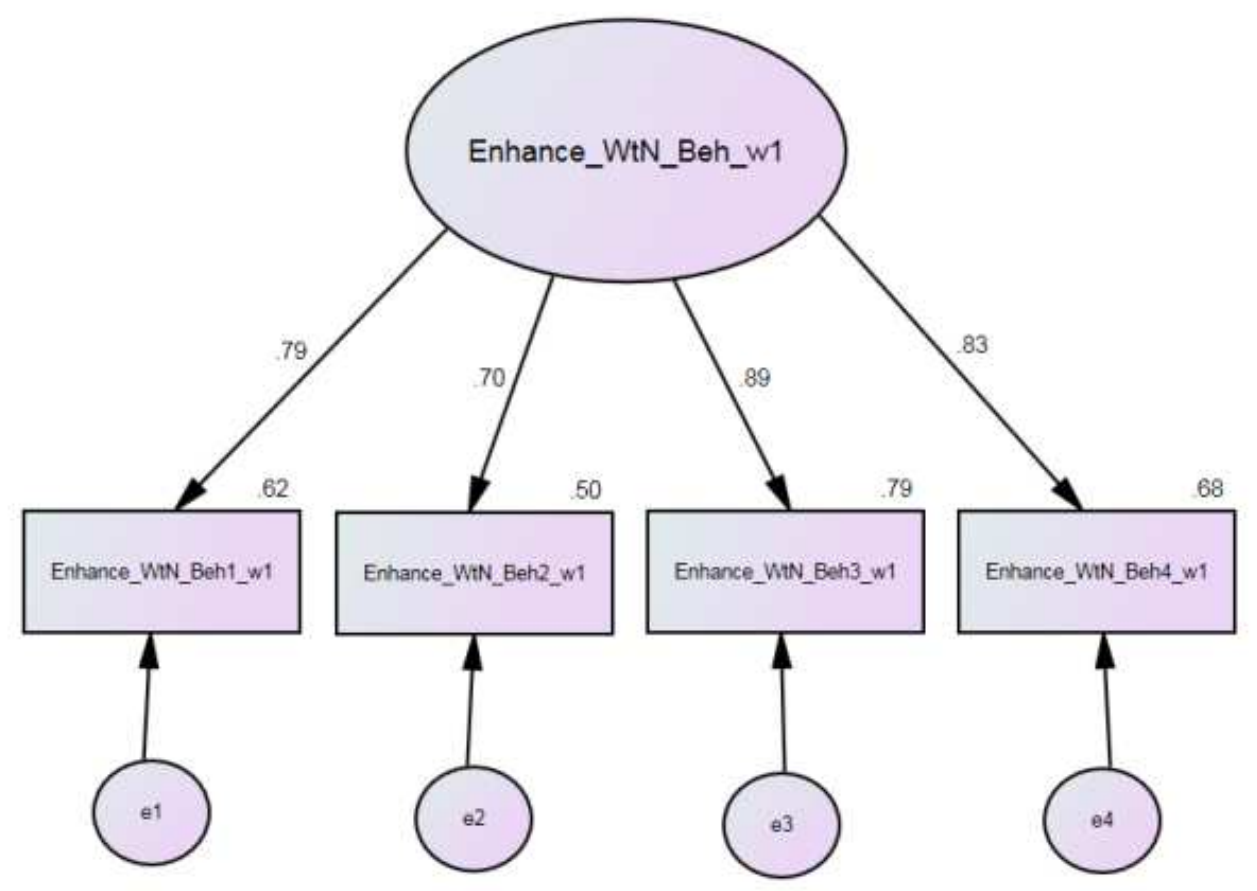

Fit Statistics:

- $\chi^{2}(2)=11.267, p>.001$

- $\mathrm{CFI}=.985$

- $\mathrm{GFI}=.982$

- RMSEA = .126

- Highest absolute value of the standardized residual covariances $=.522$ Conclusion: Adequate fit 


\section{Work-to-Nonwork Affective Enhancement}

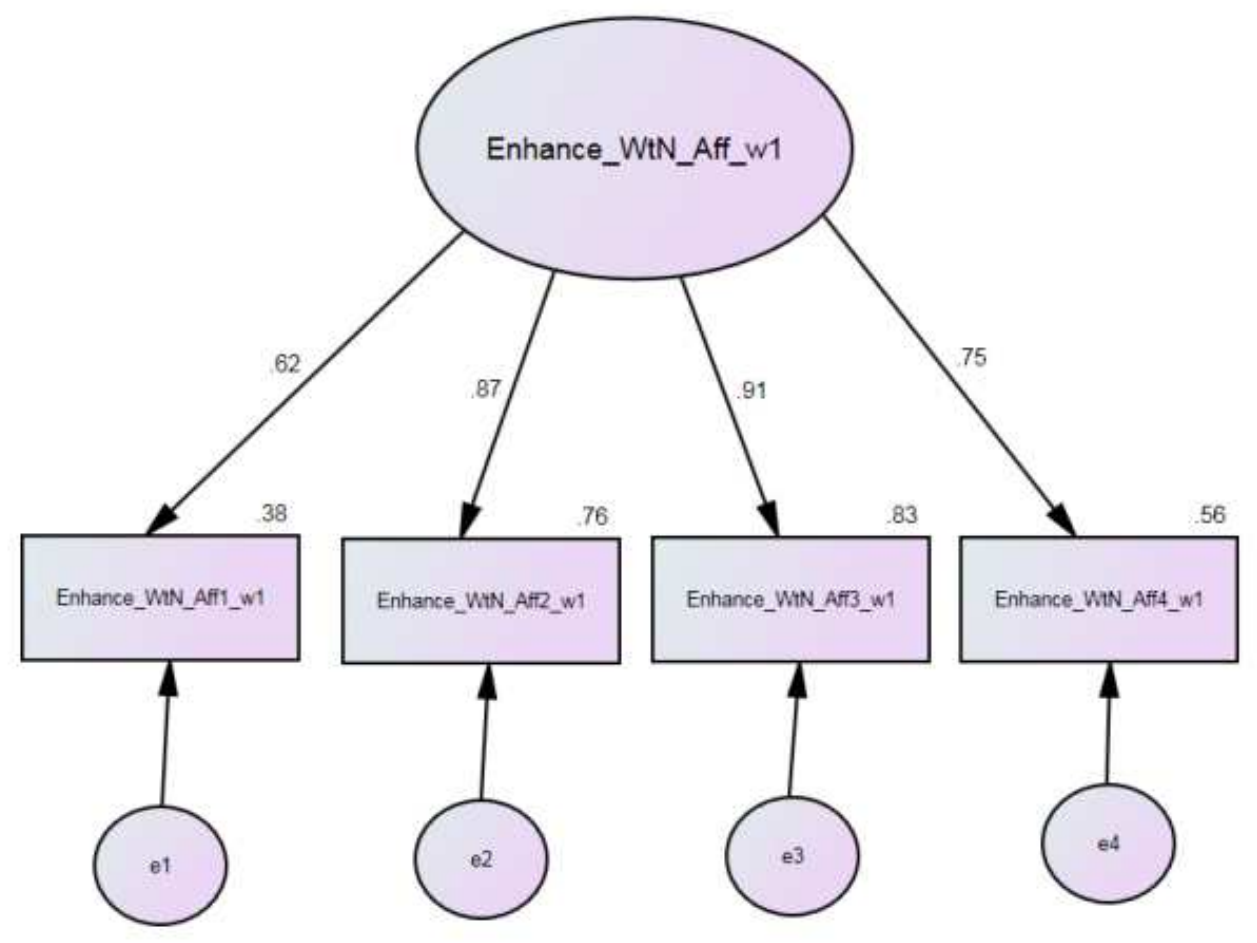

Fit Statistics:

- $\chi^{2}(2)=5.809, p>.05$

- $\mathrm{CFI}=. .994$

- $\mathrm{GFI}=.991$

- RMSEA $=.081$

- Highest absolute value of the standardized residual covariances $=.700$ Conclusion: Adequate fit 


\section{Nonwork-to-Work Behavior-Based Enhancement}

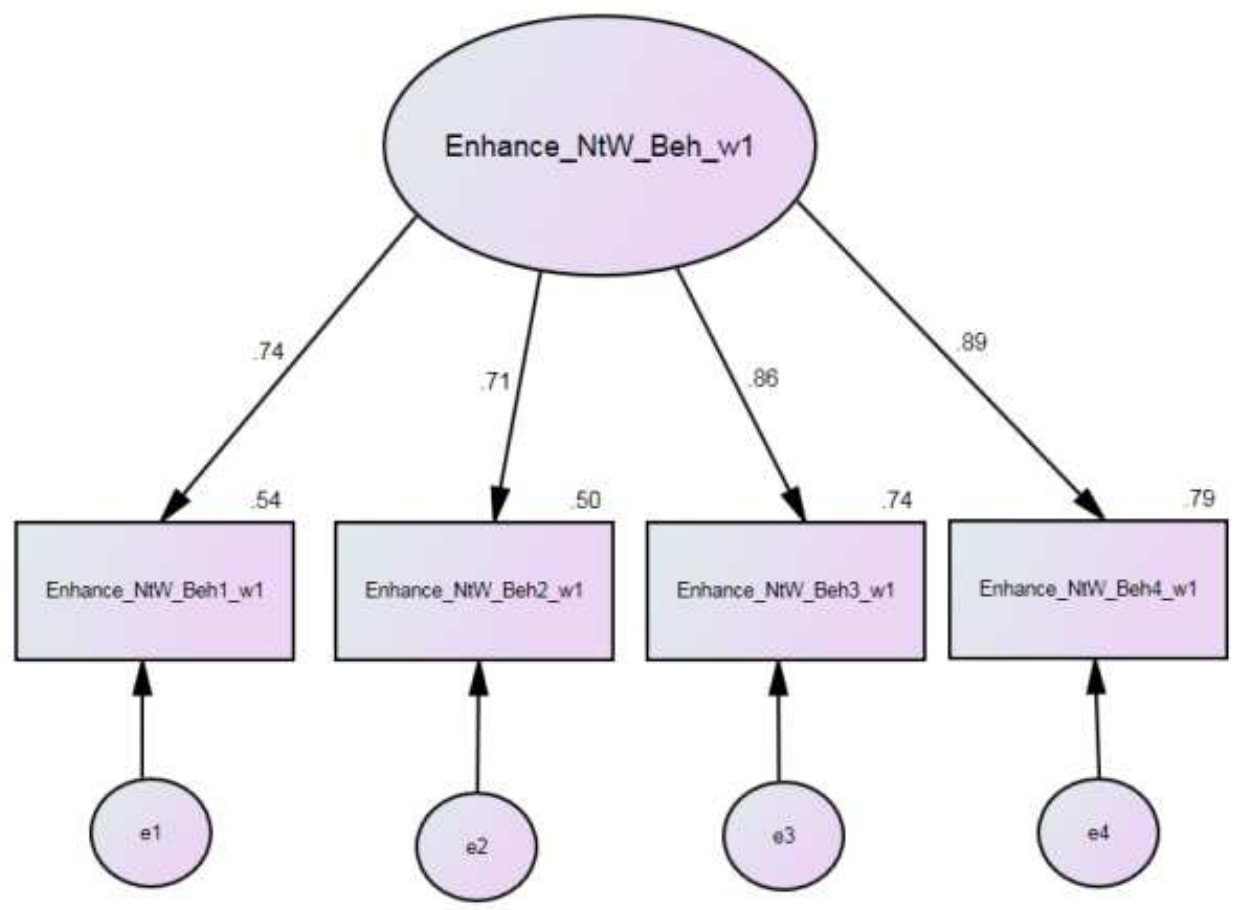

Fit Statistics:

- $\chi^{2}(2)=16.053, p<.001$

- $\mathrm{CFI}=.977$

- $\mathrm{GFI}=.975$

- RMSEA = .156

- Highest absolute value of the standardized residual covariances $=.949$ Conclusion: Adequate fit 


\section{Nonwork-to-Work Affective Enhancement}

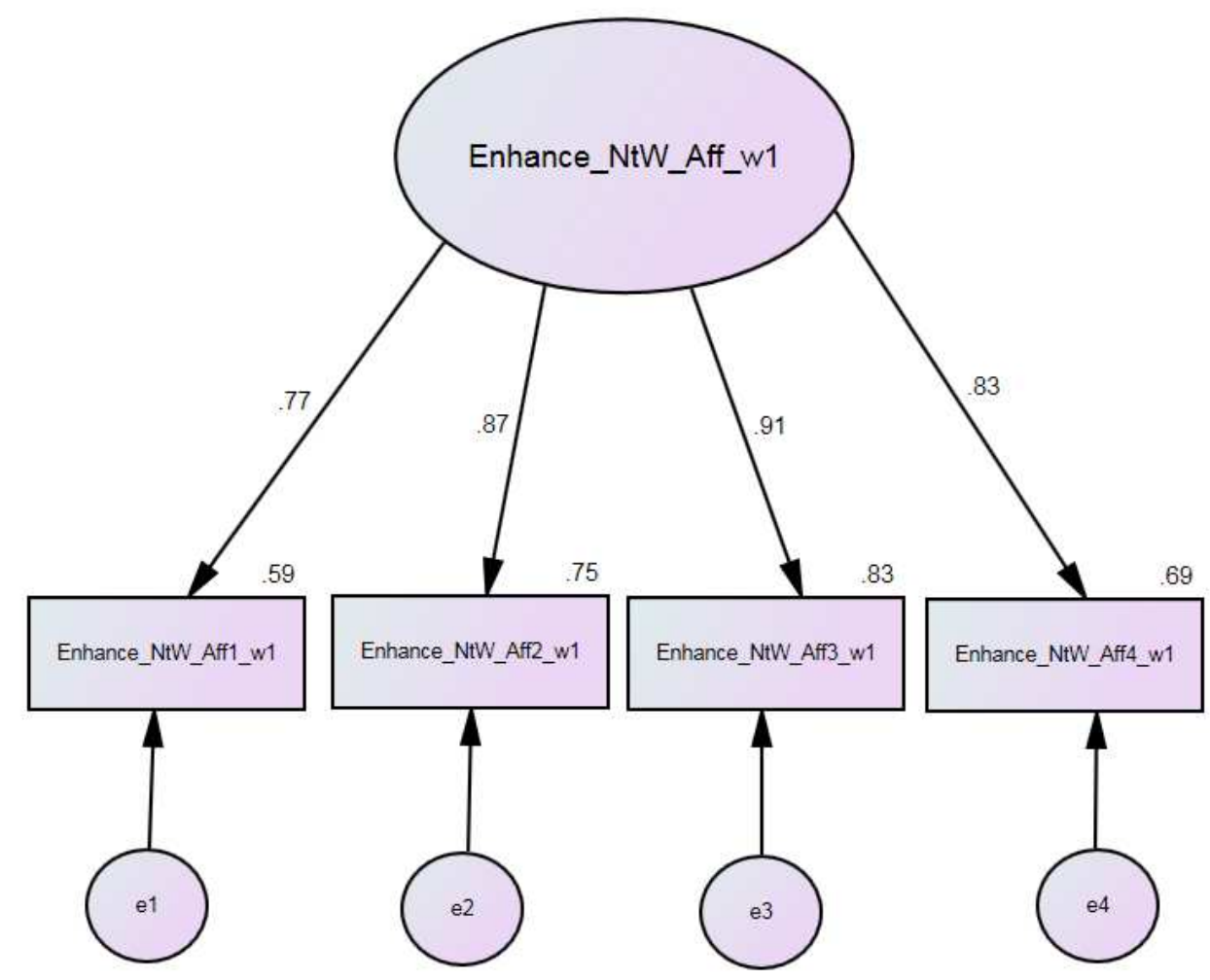

Fit Statistics:

- $\chi^{2}(2)=7.747, p>.01$

- $\mathrm{CFI}=.993$

- $\mathrm{GFI}=.988$

- RMSEA = .100

- Highest absolute value of the standardized residual covariances $=.340$ Conclusion: Adequate fit 


\section{Work-to-Nonwork Conflict}

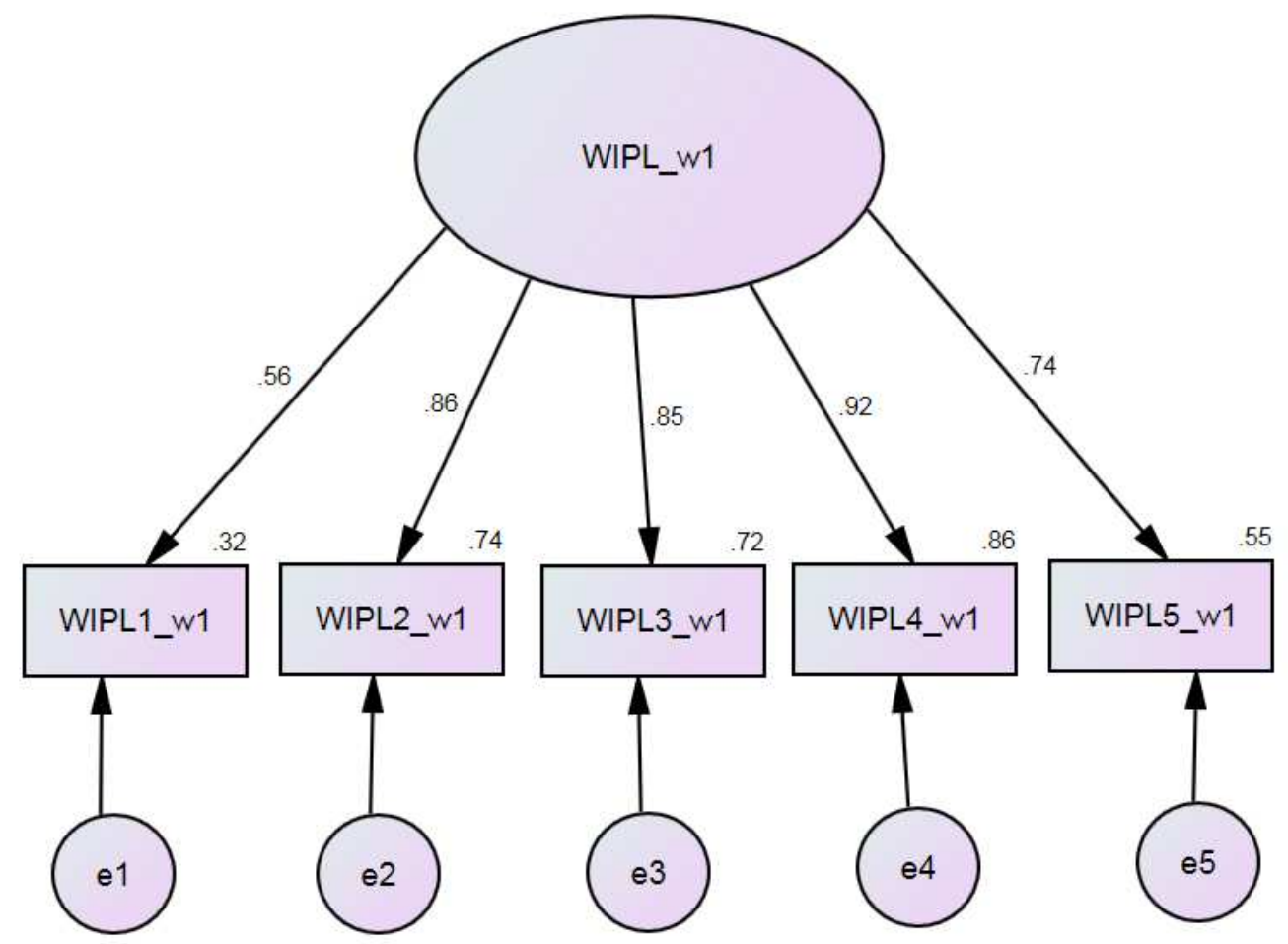

Fit Statistics:

- $\chi^{2}(5)=10.027, p>.01$

- $\mathrm{CFI}=.994$

- $\mathrm{GFI}=.986$

- RMSEA = .059

- Highest absolute value of the standardized residual covariances $=.605$ Conclusion: Adequate fit 


\section{Nonwork-to Work Conflict}

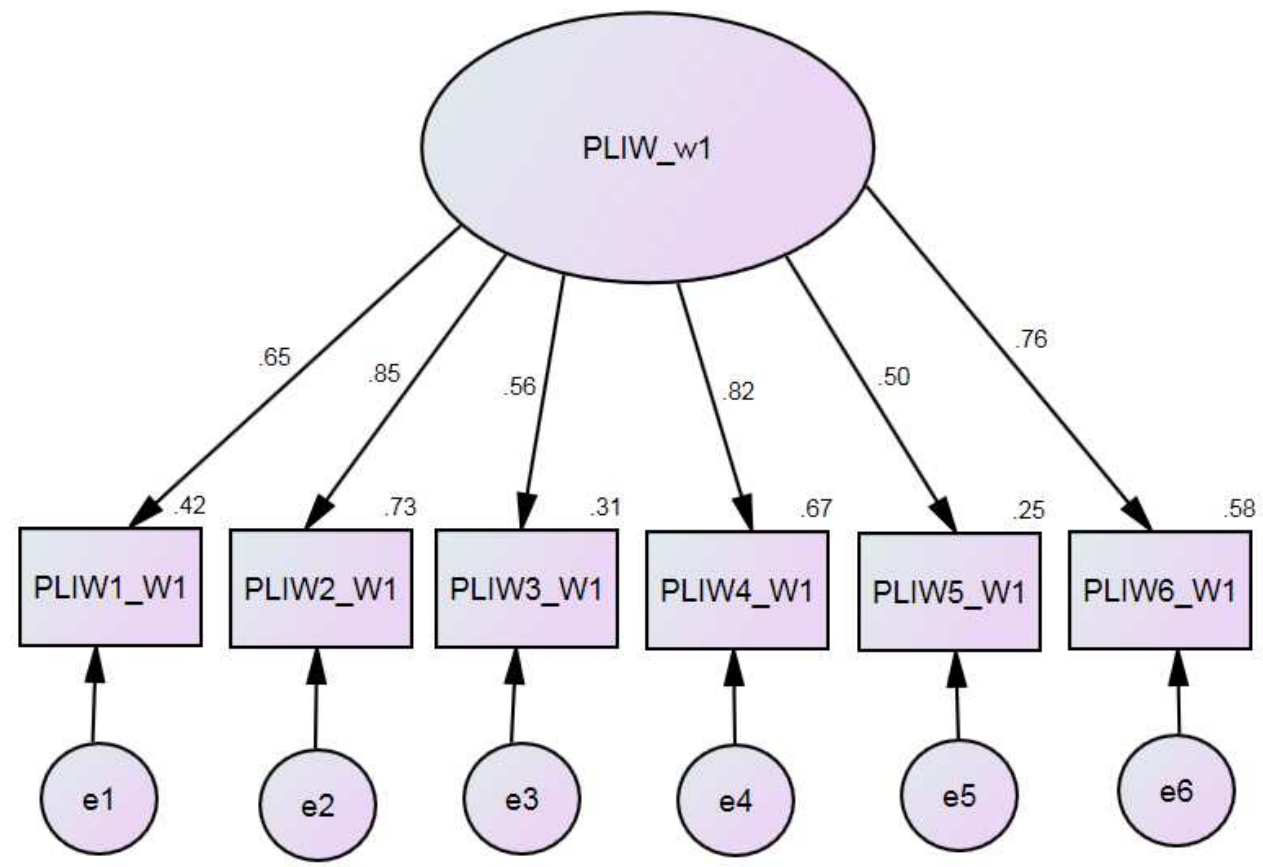

Fit Statistics:

- $\chi^{2}(9)=28.742, p>.001$

- $\mathrm{CFI}=.971$

- $\mathrm{GFI}=.969$

- RMSEA = .087

- Highest absolute value of the standardized residual covariances $=1.571$ Conclusion: Adequate fit 


\section{Appendix F: CFA Results with Bootstrapping}

\section{Boundary Preferences:}

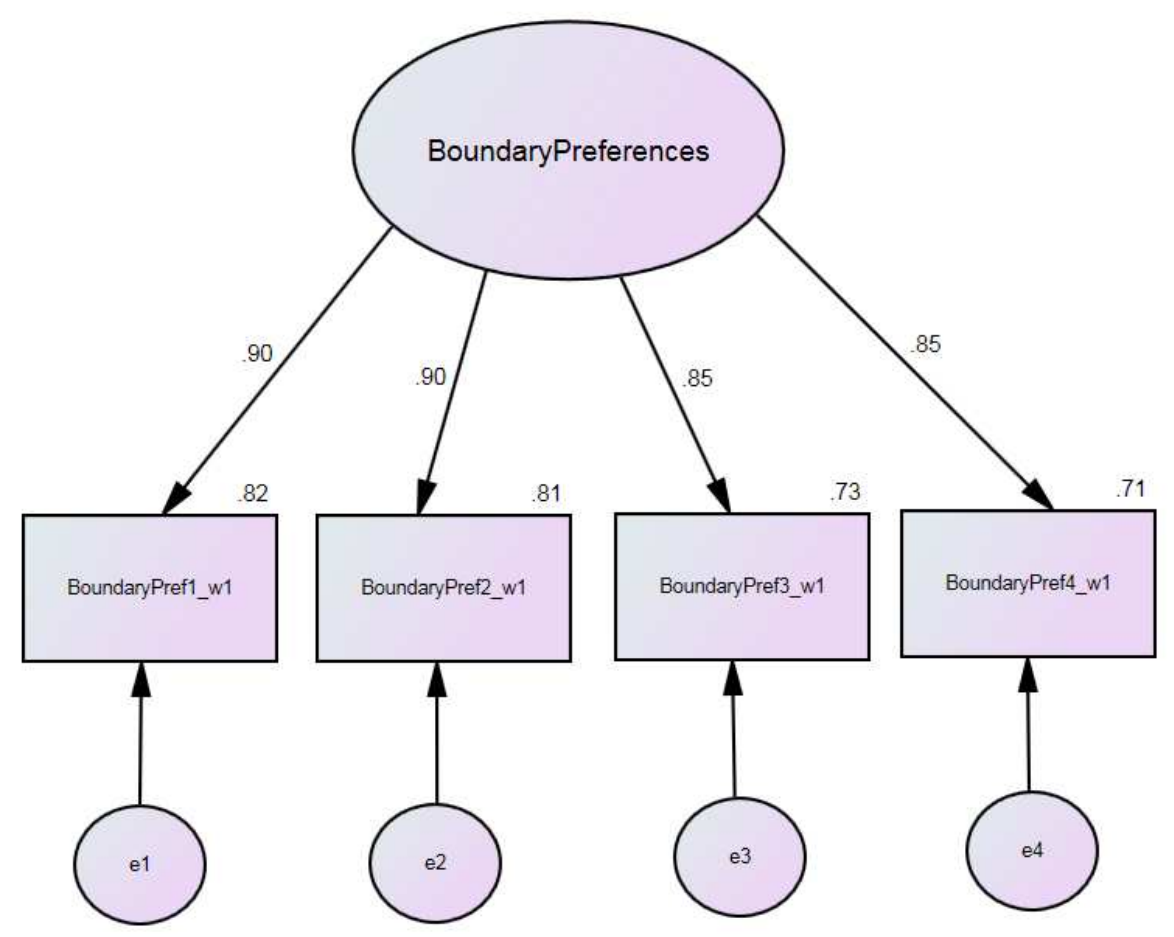

Fit Statistics:

- $\chi^{2}(2)=11.500, p>.001$

- $\mathrm{CFI}=.990$

- $\mathrm{GFI}=.980$

- RMSEA = .128

- Highest absolute value of the standardized residual covariances $=.533$ Conclusion: Adequate fit 


\section{Boundary Supplies}

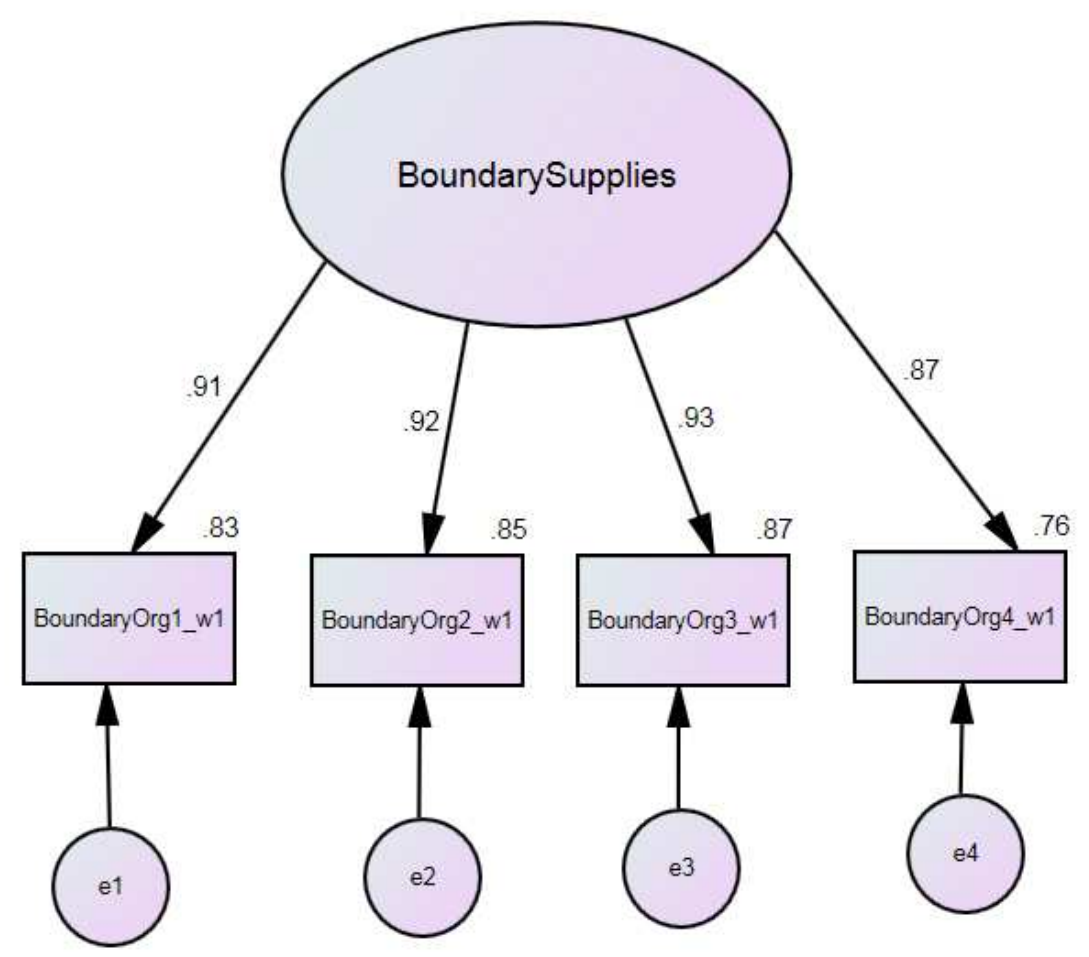

Fit Statistics:

- $\chi^{2}(2)=9.127, p>.001$

- $\mathrm{CFI}=.994$

- $\mathrm{GFI}=.985$

- RMSEA = .111

- Highest absolute value of the standardized residual covariances $=.255$

Conclusion: Adequate fit 


\section{Work-to-Nonwork Behavior-Based Enhancement}

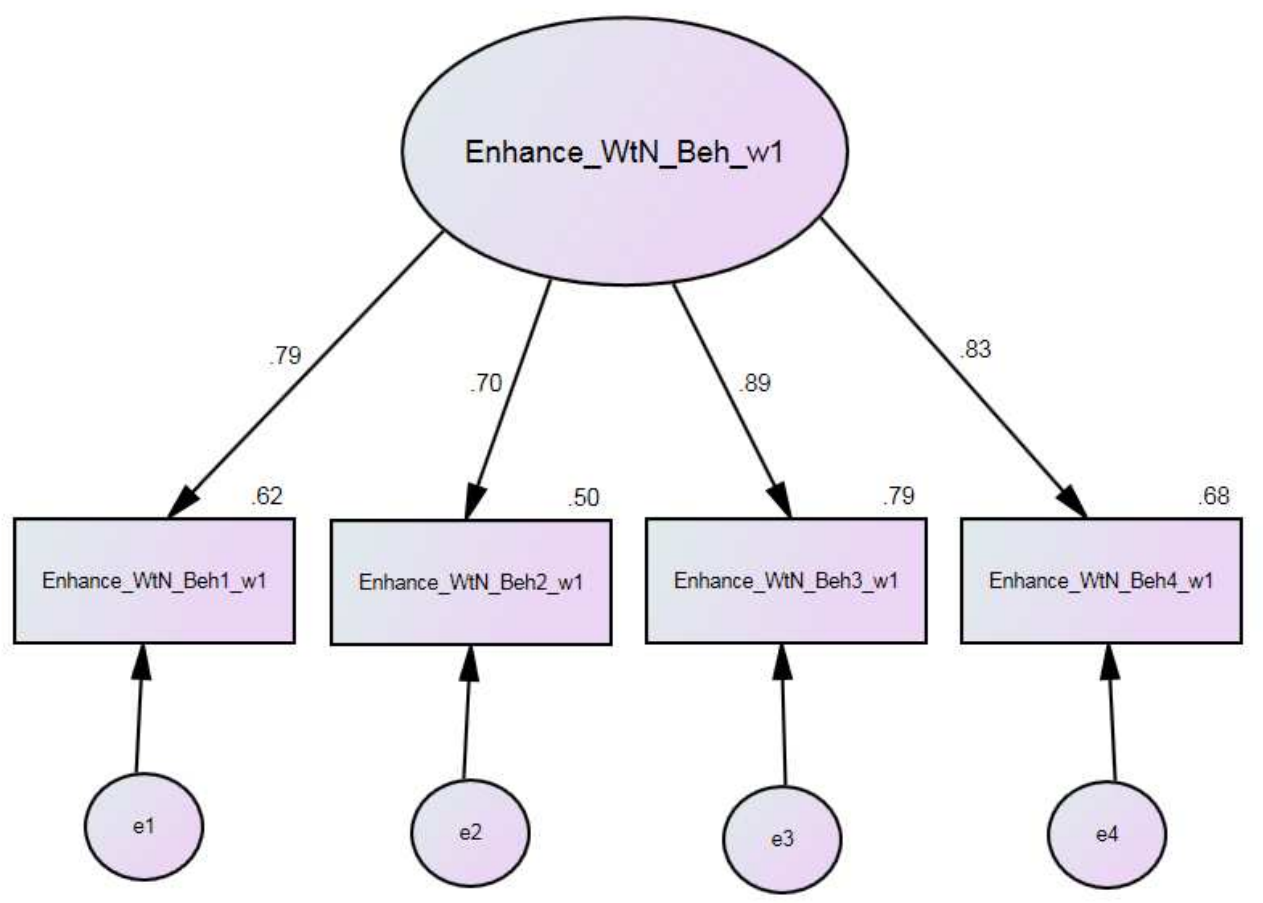

Fit Statistics:

- $\chi^{2}(2)=11.267, p>.001$

- $\mathrm{CFI}=.985$

- $\mathrm{GFI}=.982$

- RMSEA = .126

- Highest absolute value of the standardized residual covariances $=.522$ Conclusion: Adequate fit 


\section{Work-to-Nonwork Affective Enhancement}

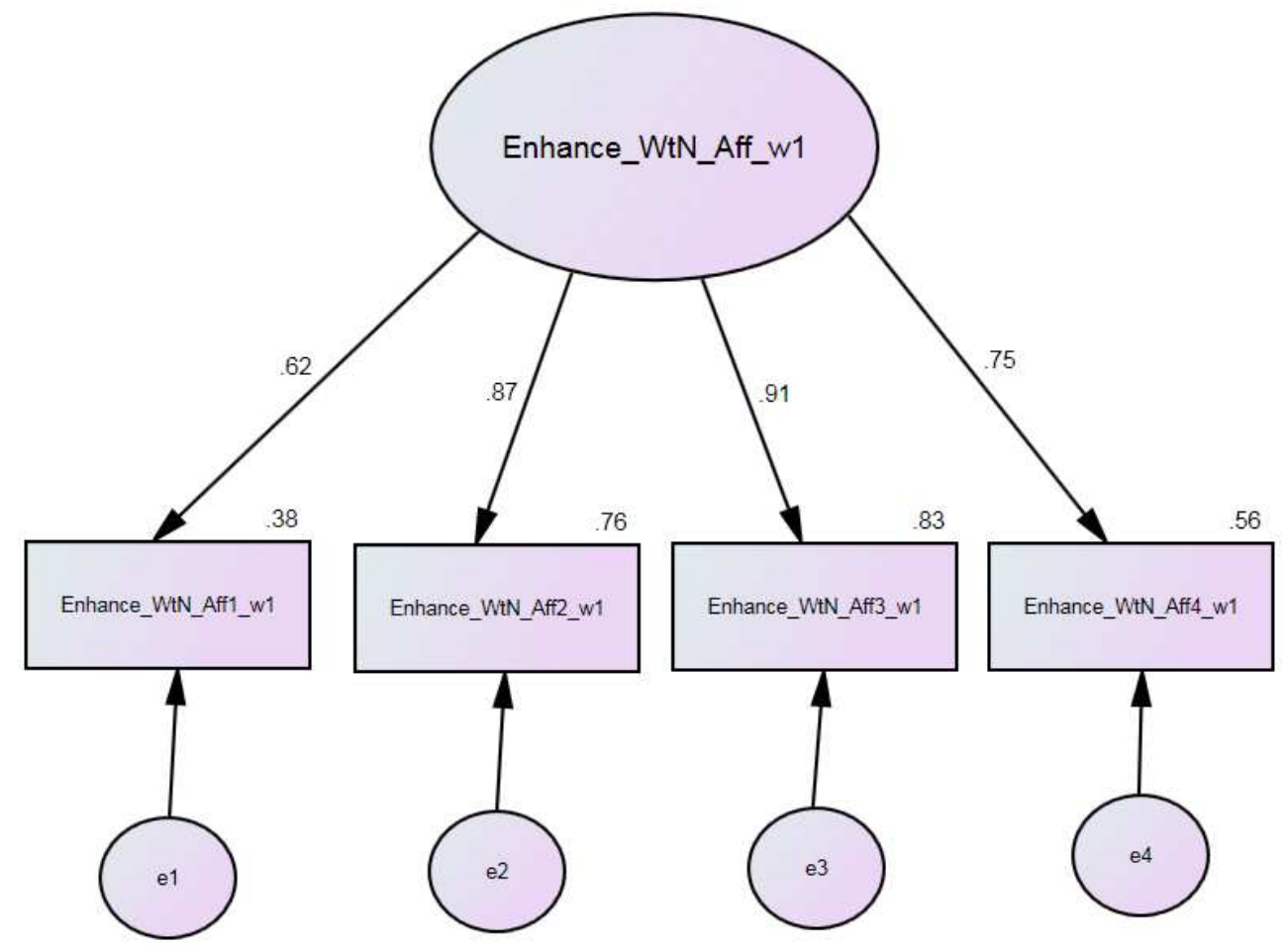

Fit Statistics:

- $\chi^{2}(2)=5.809, p>.05$

- $\mathrm{CFI}=.994$

- $\mathrm{GFI}=.991$

- RMSEA = .081

- Highest absolute value of the standardized residual covariances $=.700$ Conclusion: Adequate fit 


\section{Nonwork-to-Work Behavior-Based Enhancement}

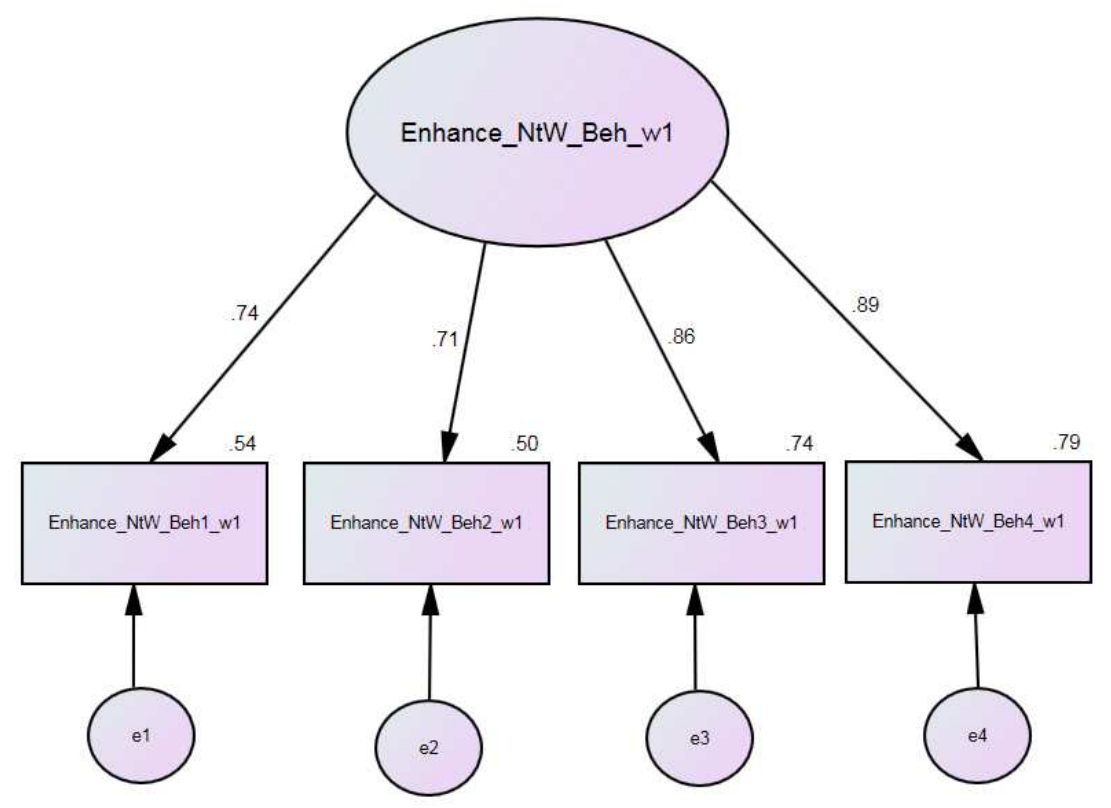

Fit Statistics:

- $\chi^{2}(2)=16.053, \mathrm{p}<.001$

- $\mathrm{CFI}=.977$

- $\mathrm{GFI}=.975$

- RMSEA = .156

- Highest absolute value of the standardized residual covariances $=.949$

Conclusion: Adequate fit 


\section{Nonwork-to-Work Affective Enhancement}

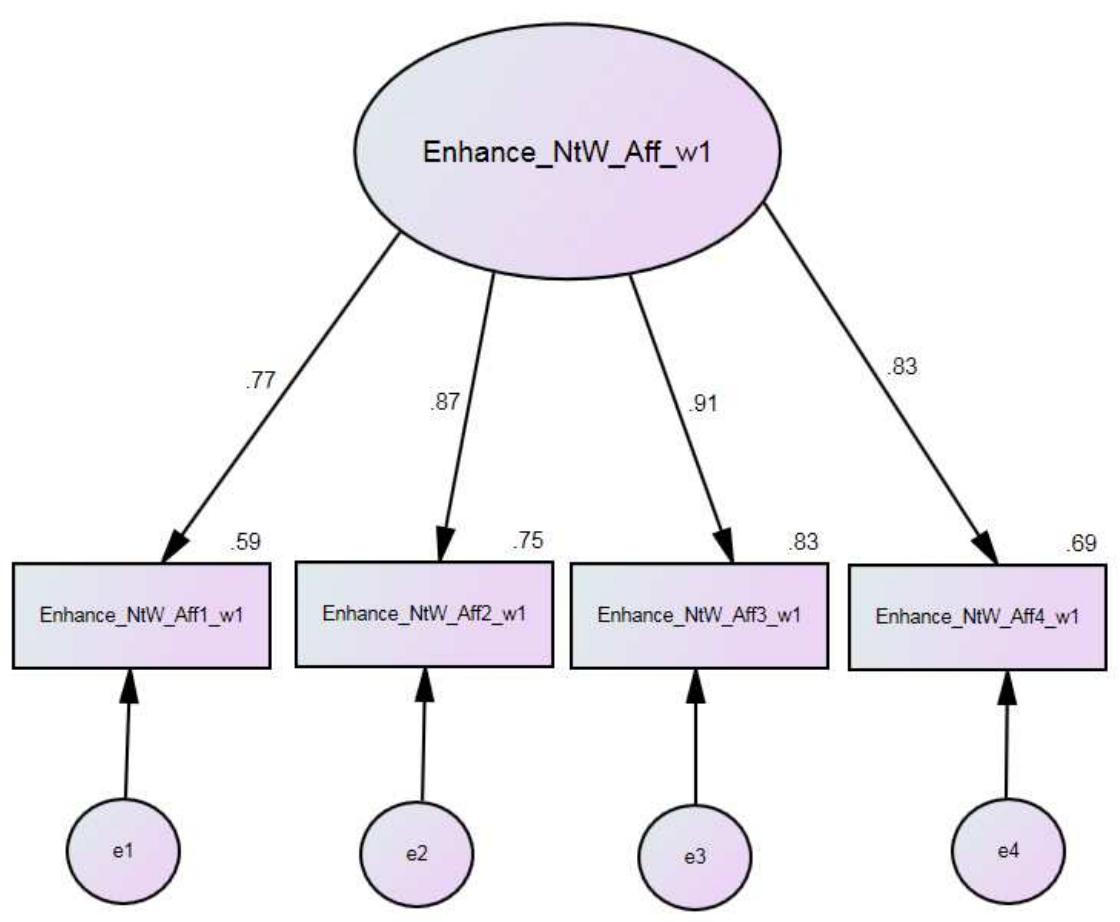

Fit Statistics:

- $\chi^{2}(2)=7.747, p>.01$

- $\mathrm{CFI}=.993$

- $\mathrm{GFI}=.988$

- RMSEA =.100

- Highest absolute value of the standardized residual covariances $=.340$

Conclusion: Adequate fit 


\section{Work-to-Nonwork Conflict}

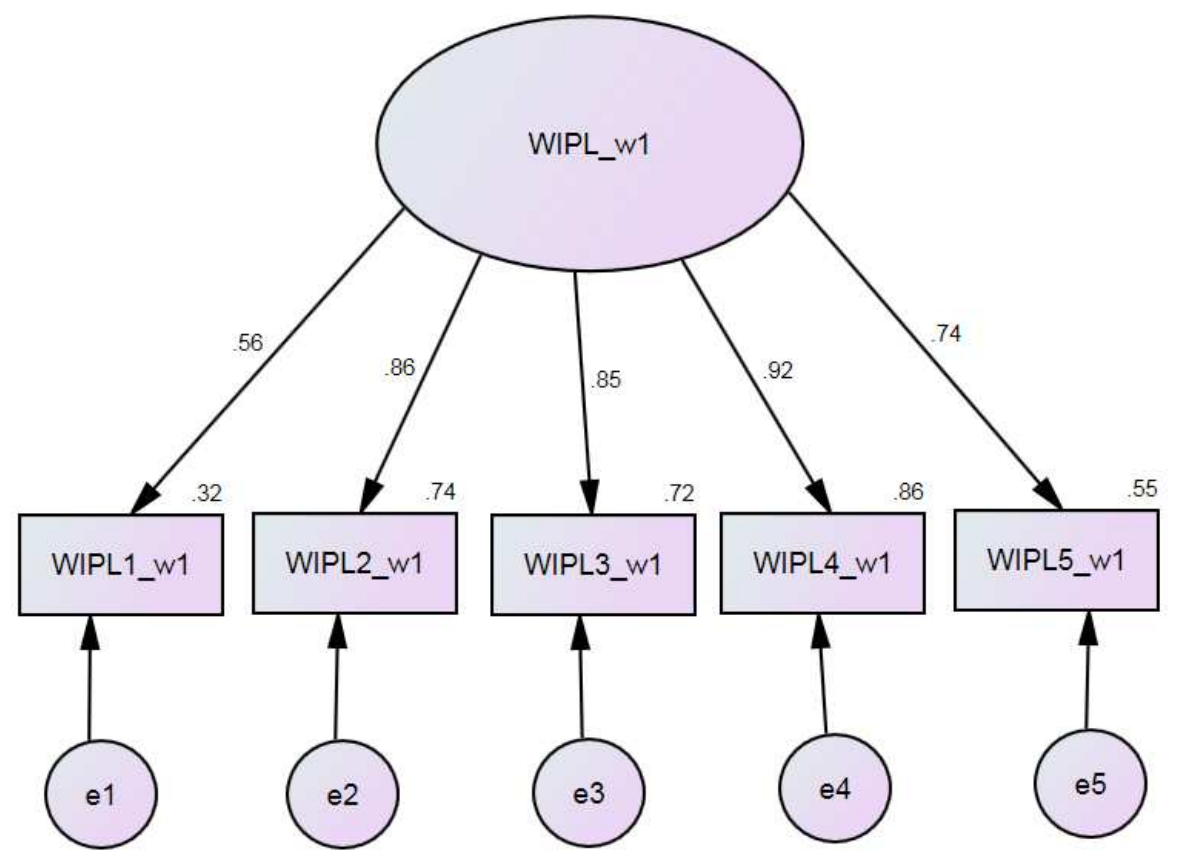

Fit Statistics:

- $\chi^{2}(5)=10.027, \mathrm{p}>.05$

- $\mathrm{CFI}=.994$

- $\mathrm{GFI}=.986$

- RMSEA = .059

- Highest absolute value of the standardized residual covariances $=.605$ Conclusion: Adequate fit 


\section{Nonwork-to-Work Conflict}

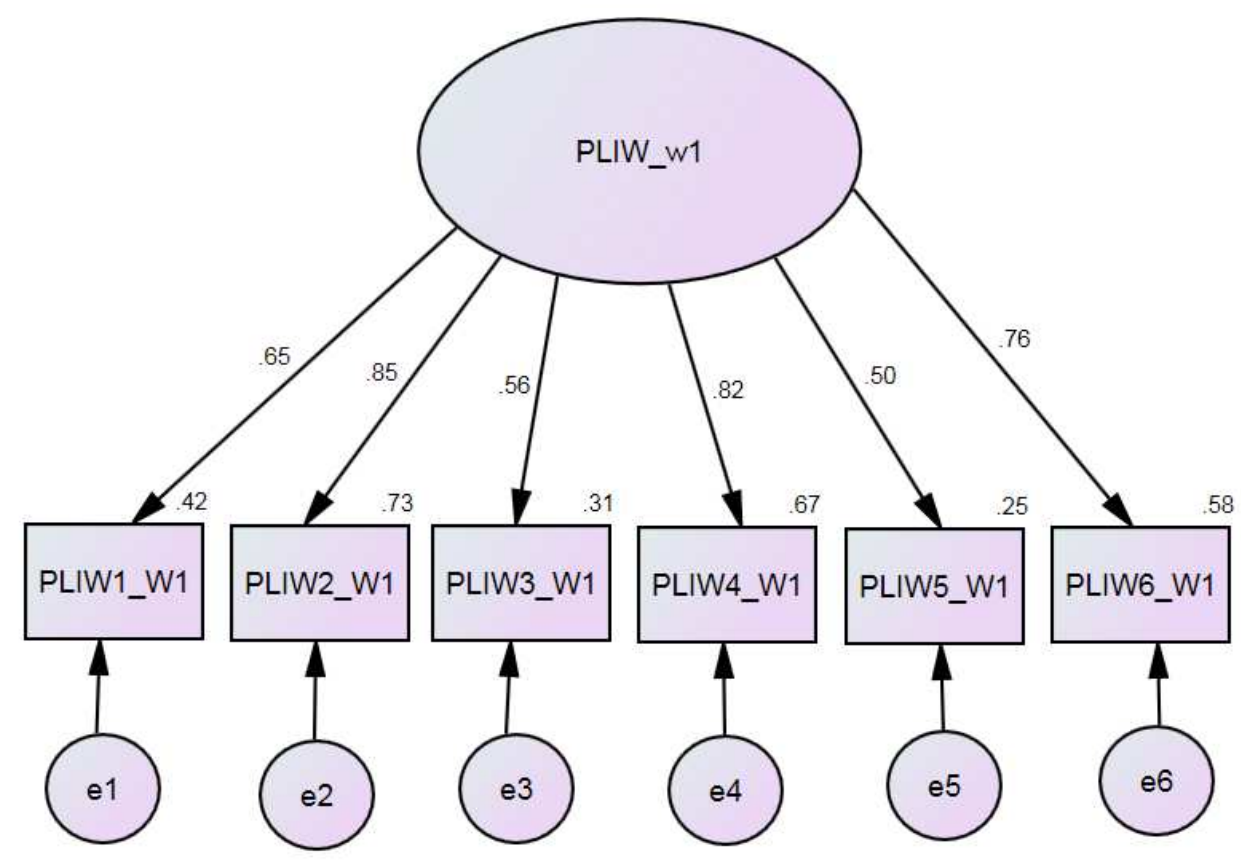

Fit Statistics:

- $\chi^{2}(9)=28.742, p>.01$

- $\mathrm{CFI}=.971$

- $\mathrm{GFI}=.969$

- RMSEA = .087

- Highest absolute value of the standardized residual covariances $=1.571$ Conclusion: Adequate fit 


\section{Appendix G. LCM Results}

Model 1: PLIW, WtN Beh, MCS

Nonwork-to-work conflict, work-to-nonwork behavior-based enhancement, and mental health

\section{Unstandardized Output:}

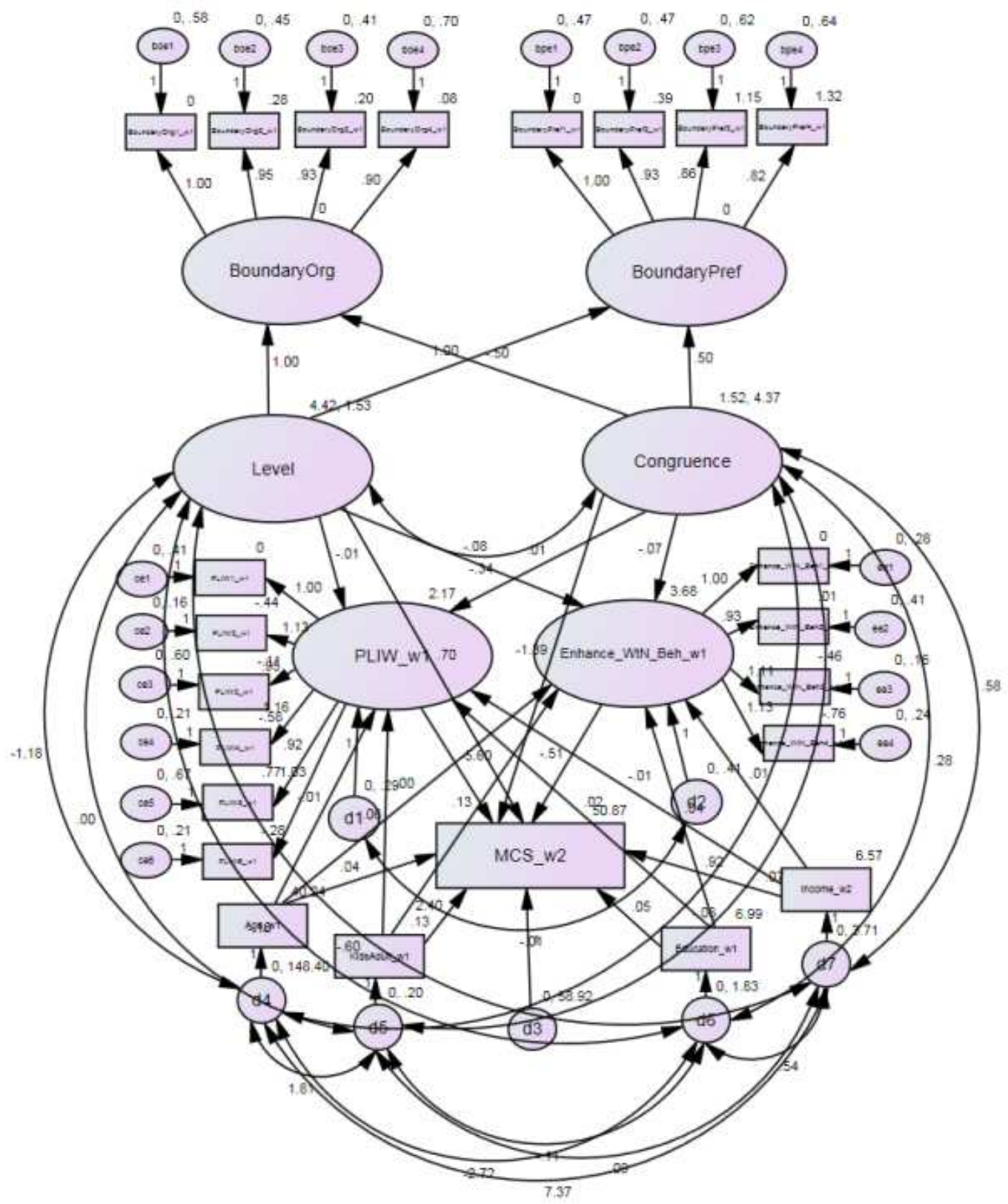

Fit Statistics 
- $\chi^{2}(199)=294.017, \mathrm{p}<.001$

- CFI: .976

- RMSEA: .039 
Model 1

\begin{tabular}{|c|c|c|c|c|c|c|c|}
\hline & & & Est. & S.E. & C.R. & $\mathrm{p}$ & St. Est. \\
\hline Enhance_WtN_Beh_w1 & $<--$ & Age_w1 & 0.004 & 0.004 & 1.019 & 0.308 & 0.068 \\
\hline MCS_w2 & $<--$ & Age_w1 & 0.045 & 0.042 & 1.061 & 0.289 & 0.06 \\
\hline PLIW_w1 & $<--$ & Age_w1 & -0.006 & 0.003 & -1.976 & 0.048 & -0.137 \\
\hline BoundaryOrg1_w1 & $<--$ & BoundaryOrg & 1 & & & & 0.915 \\
\hline BoundaryOrg2_w1 & $<--$ & BoundaryOrg & 0.946 & 0.034 & 27.969 & $* * *$ & 0.925 \\
\hline BoundaryOrg3_w1 & $<--$ & BoundaryOrg & 0.931 & 0.033 & 28.268 & $* * *$ & 0.928 \\
\hline BoundaryOrg4_w1 & $<--$ & BoundaryOrg & 0.896 & 0.037 & 24.331 & $* * *$ & 0.879 \\
\hline BoundaryPref1_w1 & $<--$ & BoundaryPref & 1 & & & & 0.91 \\
\hline BoundaryPref2_w1 & $<--$ & BoundaryPref & 0.935 & 0.038 & 24.314 & $* * *$ & 0.899 \\
\hline BoundaryPref3_w1 & $<--$ & BoundaryPref & 0.856 & 0.039 & 21.709 & $* * *$ & 0.854 \\
\hline BoundaryPref4_w1 & $<--$ & BoundaryPref & 0.822 & 0.039 & 20.96 & $* * *$ & 0.84 \\
\hline BoundaryOrg & $<--$ & Congruence & -0.5 & & & & -0.607 \\
\hline BoundaryPref & $<--$ & Congruence & 0.5 & & & & 0.693 \\
\hline Enhance_WtN_Beh_w1 & $<--$ & Congruence & -0.075 & 0.02 & -3.766 & $* * *$ & -0.234 \\
\hline MCS_w2 & $<--$ & Congruence & -1.389 & 0.233 & -5.966 & $* * *$ & -0.319 \\
\hline PLIW_w1 & $<--$ & Congruence & 0.013 & 0.016 & 0.799 & 0.424 & 0.051 \\
\hline Enhance_WtN_Beh_w1 & $<--$ & Education_w1 & 0.042 & 0.031 & 1.368 & 0.171 & 0.084 \\
\hline MCS_w2 & $<-$ & Education_w1 & 0.052 & 0.35 & 0.147 & 0.883 & 0.008 \\
\hline PLIW_w1 & $<--$ & Education_w1 & 0.021 & 0.026 & 0.813 & 0.416 & 0.052 \\
\hline Enhance_WtN_Beh1_w1 & $<--$ & Enhance_WtN_Beh_w1 & 1 & & & & 0.787 \\
\hline Enhance_WtN_Beh2_w1 & $<--$ & Enhance_WtN_Beh_w1 & 0.928 & 0.073 & 12.696 & $* * *$ & 0.697 \\
\hline Enhance_WtN_Beh3_w1 & $<--$ & Enhance_WtN_Beh_w1 & 1.114 & 0.067 & 16.533 & $* * *$ & 0.883 \\
\hline Enhance_WtN_Beh4_w1 & $<--$ & Enhance_WtN_Beh_w1 & 1.127 & 0.071 & 15.782 & $* * *$ & 0.839 \\
\hline MCS_w2 & $<--$ & Enhance_WtN_Beh_w1 & -0.509 & 0.732 & -0.695 & 0.487 & -0.038 \\
\hline Enhance_WtN_Beh_w1 & $<--$ & Income_w2 & 0.005 & 0.023 & 0.226 & 0.821 & 0.015 \\
\hline MCS_w2 & $<--$ & Income_w2 & 0.916 & 0.261 & 3.503 & $* * *$ & 0.194 \\
\hline PLIW_w1 & $<--$ & Income_w2 & -0.006 & 0.019 & -0.32 & 0.749 & -0.022 \\
\hline Enhance_WtN_Beh_w1 & $<--$ & KidsAdult_w1 & 0.134 & 0.097 & 1.392 & 0.164 & 0.089 \\
\hline MCS_w2 & $<--$ & KidsAdult_w1 & 2.403 & 1.108 & 2.169 & 0.03 & 0.117 \\
\hline PLIW_w1 & $<--$ & KidsAdult_w1 & 0.056 & 0.081 & 0.691 & 0.489 & 0.046 \\
\hline BoundaryOrg & $<--$ & Level & 1 & & & & 0.718 \\
\hline BoundaryPref & $<--$ & Level & 1 & & & & 0.819 \\
\hline Enhance_WtN_Beh_w1 & $<--$ & Level & -0.083 & 0.034 & -2.47 & 0.013 & -0.153 \\
\hline MCS_w2 & $<--$ & Level & 0.697 & 0.389 & 1.791 & 0.073 & 0.095 \\
\hline PLIW_w1 & $<--$ & Level & -0.011 & 0.028 & -0.381 & 0.703 & -0.024 \\
\hline MCS_w2 & $<--$ & PLIW_w1 & -5.602 & 0.961 & -5.83 & $* * *$ & -0.334 \\
\hline PLIW1_w1 & $<--$ & PLIW_w1 & 1 & & & & 0.648 \\
\hline PLIW2_w1 & $<--$ & PLIW_w1 & 1.133 & 0.094 & 12.01 & $* * *$ & 0.838 \\
\hline PLIW3_w1 & $<--$ & PLIW_w1 & 0.95 & 0.11 & 8.639 & $* * *$ & 0.555 \\
\hline PLIW4_w1 & $<--$ & PLIW_w1 & 1.163 & 0.099 & 11.741 & $* * *$ & 0.81 \\
\hline PLIW5_w1 & $<--$ & PLIW_w1 & 0.924 & 0.113 & 8.171 & $* * *$ & 0.521 \\
\hline PLIW6_w1 & $<--$ & PLIW_w1 & 1.028 & 0.091 & 11.334 & $* * *$ & 0.772 \\
\hline
\end{tabular}




\section{Model 2: PLIW, WtN Beh, PCS}

Nonwork-to-work conflict, work-to-nonwork behavior-based enhancement, and physical health

Unstandardized Output:

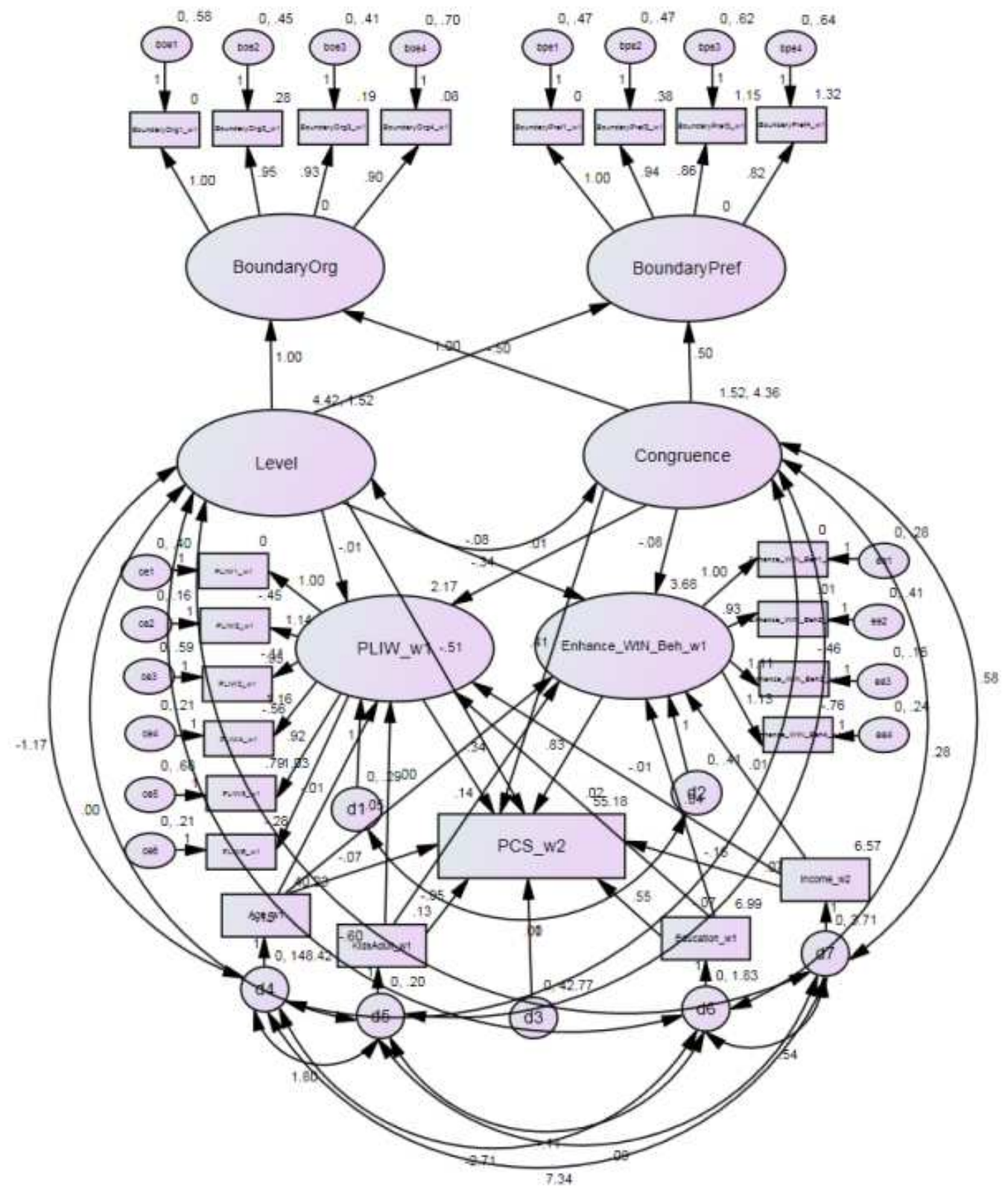

Fit Statistics:

- $\chi^{2}(199)=303.623, p<.001$

- $\quad$ CFI: .973

- RMSEA: .041 
Model 2 Output

\begin{tabular}{|c|c|c|c|c|c|c|c|}
\hline & & & Est. & S.E. & C.R. & $\mathrm{p}$ & $\begin{array}{l}\text { Std. } \\
\text { Est. }\end{array}$ \\
\hline Enhance_WtN_Beh_w1 & $\begin{array}{l}<- \\
\end{array}$ & Age_w1 & 0.004 & 0.004 & 0.996 & 0.319 & 0.066 \\
\hline PCS_w2 & $<-$ & Age_w1 & -0.069 & 0.035 & -1.952 & 0.051 & -0.123 \\
\hline PLIW_w1 & $<-$ & Age_w1 & -0.006 & 0.003 & -1.954 & 0.051 & -0.135 \\
\hline BoundaryOrg1_w1 & $<-$ & BoundaryOrg & 1 & & & & 0.914 \\
\hline BoundaryOrg2_w1 & $<-$ & BoundaryOrg & 0.947 & 0.034 & 27.875 & 0.000 & 0.925 \\
\hline BoundaryOrg3_w1 & $<-$ & BoundaryOrg & 0.933 & 0.033 & 28.218 & 0.000 & 0.929 \\
\hline BoundaryOrg4_w1 & $<-$ & BoundaryOrg & 0.898 & 0.037 & 24.305 & 0.000 & 0.879 \\
\hline BoundaryPref1_w1 & $<-$ & BoundaryPref & 1 & & & & 0.910 \\
\hline BoundaryPref2_w1 & $<-$ & BoundaryPref & 0.936 & 0.038 & 24.328 & 0.000 & 0.900 \\
\hline BoundaryPref3_w1 & $<-$ & BoundaryPref & 0.857 & 0.040 & 21.668 & 0.000 & 0.854 \\
\hline BoundaryPref4_w1 & $<-$ & BoundaryPref & 0.822 & 0.039 & 20.922 & 0.000 & 0.840 \\
\hline BoundaryOrg & $<--$ & Congruence & -0.500 & & & & -0.607 \\
\hline BoundaryPref & $<-$ & Congruence & 0.500 & & & & 0.693 \\
\hline Enhance_WtN_Beh_w1 & $<--$ & Congruence & -0.075 & 0.020 & -3.774 & 0.000 & -0.234 \\
\hline PCS_w2 & $<--$ & Congruence & 0.411 & 0.195 & 2.111 & 0.035 & 0.126 \\
\hline PLIW_w1 & $<--$ & Congruence & 0.013 & 0.016 & 0.793 & 0.428 & 0.050 \\
\hline Enhance_WtN_Beh_w1 & $<-$ & Education_w1 & 0.042 & 0.031 & 1.373 & 0.170 & 0.085 \\
\hline PCS_w2 & $<-$ & Education_w1 & 0.553 & 0.294 & 1.883 & 0.060 & 0.110 \\
\hline PLIW_w1 & $<--$ & Education_w1 & 0.020 & 0.026 & 0.789 & 0.430 & 0.050 \\
\hline Enhance_WtN_Beh1_w1 & $<-$ & Enhance_WtN_Beh_w1 & 1 & & & & 0.787 \\
\hline Enhance_WtN_Beh2_w1 & $<-$ & Enhance_WtN_Beh_w1 & 0.929 & 0.073 & 12.708 & 0.000 & 0.697 \\
\hline Enhance_WtN_Beh3_w1 & $<-$ & Enhance_WtN_Beh_w1 & 1.114 & 0.067 & 16.54 & 0.000 & 0.883 \\
\hline Enhance_WtN_Beh4_w1 & $<--$ & Enhance_WtN_Beh_w1 & 1.126 & 0.071 & 15.778 & 0.000 & 0.838 \\
\hline PCS_w2 & $<-$ & Enhance_WtN_Beh_w1 & 0.826 & 0.616 & 1.341 & 0.180 & 0.082 \\
\hline Enhance_WtN_Beh_w1 & $<-$ & Income_w2 & 0.006 & 0.023 & 0.262 & 0.793 & 0.017 \\
\hline PCS_w2 & $<-$ & Income_w2 & -0.155 & 0.220 & -0.706 & 0.480 & -0.044 \\
\hline PLIW_w1 & $<-$ & Income_w2 & -0.005 & 0.019 & -0.283 & 0.777 & -0.019 \\
\hline Enhance_WtN_Beh_w1 & $<-$ & KidsAdult_w1 & 0.141 & 0.097 & 1.462 & 0.144 & 0.093 \\
\hline PCS_w2 & $<-$ & KidsAdult_w1 & -0.946 & 0.930 & -1.017 & 0.309 & -0.062 \\
\hline PLIW_w1 & $<--$ & KidsAdult_w1 & 0.052 & 0.081 & 0.641 & 0.521 & 0.042 \\
\hline BoundaryOrg & $<-$ & Level & 1 & & & & 0.718 \\
\hline BoundaryPref & $<-$ & Level & 1 & & & & 0.819 \\
\hline Enhance_WtN_Beh_w1 & $<-$ & Level & -0.083 & 0.034 & -2.456 & 0.014 & -0.152 \\
\hline PCS_w2 & $<--$ & Level & -0.507 & 0.325 & -1.56 & 0.119 & -0.092 \\
\hline PLIW_w1 & $<--$ & Level & -0.010 & 0.028 & -0.373 & 0.709 & -0.024 \\
\hline PCS_w2 & $<-$ & PLIW_w1 & -0.344 & 0.738 & -0.465 & 0.642 & -0.027 \\
\hline PLIW'W1_w1 & $<-$ & PLIW_w1 & 1 & & & & 0.648 \\
\hline PLIW2_w1 & $<-$ & PLIW_w1 & 1.138 & 0.095 & 12.026 & 0.000 & 0.842 \\
\hline PLIW3_w1 & $<-$ & PLIW_w1 & 0.954 & 0.110 & 8.664 & 0.000 & 0.557 \\
\hline PLIW4_w1 & $<-$ & PLIW_w1 & 1.156 & 0.099 & 11.683 & 0.000 & 0.805 \\
\hline PLIW5_w1 & $<-$ & PLIW_w1 & 0.917 & 0.113 & 8.116 & 0.000 & 0.517 \\
\hline PLIW6_w1 & $<-$ & PLIW_w1 & 1.027 & 0.091 & 11.32 & 0.000 & 0.772 \\
\hline
\end{tabular}




\section{Model 3. WIPL, WtN Beh, PCS}

Work-to-nonwork conflict, work-to-nonwork behavior-based enhancement, and physical health

\section{Unstandardized Output:}

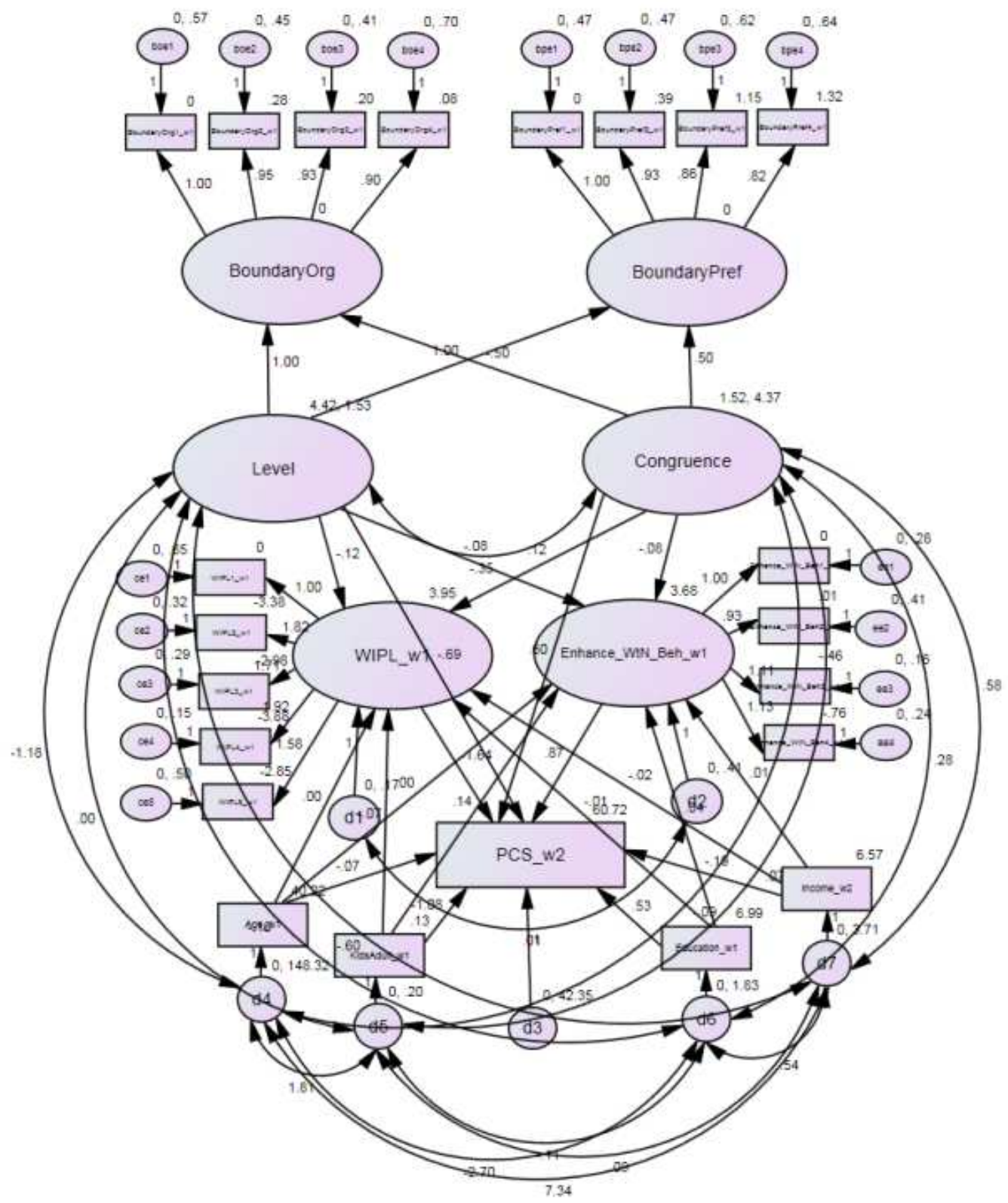

Fit Statistics:

- $\chi^{2}(178)=275.371, p<.001$

- CFI: 977

- RMSEA: .042 
Model 3 Output

\begin{tabular}{|c|c|c|c|c|c|c|c|}
\hline & & & Est. & S.E. & C.R. & $\mathrm{p}$ & $\begin{array}{l}\text { Std. } \\
\text { Est. }\end{array}$ \\
\hline Enhance_WtN_Beh_w1 & $<-$ & Age_w1 & 0.004 & 0.004 & 0.965 & 0.335 & 0.064 \\
\hline PCS_w2 & $<-$ & Age_w1 & -0.065 & 0.035 & -1.875 & 0.061 & -0.117 \\
\hline WIPL_w1 & $<-$ & Age_w1 & 0.001 & 0.002 & 0.316 & 0.752 & 0.018 \\
\hline BoundaryOrg1_w1 & $<-$ & BoundaryOrg & 1 & & & & 0.916 \\
\hline BoundaryOrg2_w1 & $<-$ & BoundaryOrg & 0.945 & 0.034 & 28.071 & 0.000 & 0.925 \\
\hline BoundaryOrg3_w1 & $<-$ & BoundaryOrg & 0.930 & 0.033 & 28.329 & 0.000 & 0.928 \\
\hline BoundaryOrg4_w1 & $<-$ & BoundaryOrg & 0.896 & 0.037 & 24.402 & 0.000 & 0.879 \\
\hline BoundaryPref1_w1 & $<-$ & BoundaryPref & 1 & & & & 0.91 \\
\hline BoundaryPref2_w1 & $<-$ & BoundaryPref & 0.935 & 0.038 & 24.307 & 0.000 & 0.899 \\
\hline BoundaryPref3_w1 & $<--$ & BoundaryPref & 0.857 & 0.039 & 21.705 & 0.000 & 0.854 \\
\hline BoundaryPref4_w1 & $<-$ & BoundaryPref & 0.822 & 0.039 & 20.961 & 0.000 & 0.84 \\
\hline BoundaryOrg & $<--$ & Congruence & -0.500 & & & & -0.607 \\
\hline BoundaryPref & $<--$ & Congruence & 0.500 & & & & 0.693 \\
\hline Enhance_WtN_Beh_w1 & $<--$ & Congruence & -0.075 & 0.020 & -3.772 & 0.000 & -0.234 \\
\hline PCS_w2 & $<-$ & Congruence & 0.600 & 0.228 & 2.628 & 0.009 & 0.184 \\
\hline WIPL_w1 & $<-$ & Congruence & 0.116 & 0.017 & 6.924 & 0.000 & 0.481 \\
\hline Enhance_WtN_Beh_w1 & $<--$ & Education_w1 & 0.042 & 0.031 & 1.363 & 0.173 & 0.084 \\
\hline PCS_w2 & $<--$ & Education_w1 & 0.530 & 0.293 & 1.81 & 0.070 & 0.106 \\
\hline WIPL_w1 & $<-$ & Education_w1 & -0.01 & 0.020 & -0.485 & 0.628 & -0.026 \\
\hline Enhance_WtN_Beh1_w1 & $<-$ & Enhance_WtN_Beh_w1 & 1 & & & & 0.787 \\
\hline Enhance_WtN_Beh2_w1 & $<-$ & Enhance_WtN_Beh_w1 & 0.929 & 0.073 & 12.705 & 0.000 & 0.697 \\
\hline Enhance_WtN_Beh3_w1 & $<--$ & Enhance_WtN_Beh_w1 & 1.114 & 0.067 & 16.546 & 0.000 & 0.884 \\
\hline Enhance_WtN_Beh4_w1 & $<-$ & Enhance_WtN_Beh_w1 & 1.126 & 0.071 & 15.775 & 0.000 & 0.838 \\
\hline PCS_w2 & $<-$ & Enhance_WtN_Beh_w1 & 0.873 & 0.614 & 1.421 & 0.155 & 0.086 \\
\hline Enhance_WtN_Beh_w1 & $<-$ & Income_w2 & 0.006 & 0.023 & 0.283 & 0.777 & 0.019 \\
\hline PCS_w2 & $<-$ & Income_w2 & -0.194 & 0.220 & -0.879 & 0.380 & -0.055 \\
\hline WIPL_w1 & $<-$ & Income_w2 & -0.024 & 0.015 & -1.599 & 0.110 & -0.091 \\
\hline Enhance_WtN_Beh_w1 & $<-$ & KidsAdult_w1 & 0.141 & 0.097 & 1.461 & 0.144 & 0.093 \\
\hline PCS_w2 & $<-$ & KidsAdult_w1 & -1.082 & 0.929 & -1.164 & 0.244 & -0.07 \\
\hline WIPL_w1 & $<-$ & KidsAdult_w1 & -0.068 & 0.063 & -1.082 & 0.279 & -0.059 \\
\hline BoundaryOrg & $<-$ & Level & 1 & & & & 0.717 \\
\hline BoundaryPref & $<-$ & Level & 1 & & & & 0.820 \\
\hline Enhance_WtN_Beh_w1 & $<-$ & Level & -0.083 & 0.034 & -2.458 & 0.014 & -0.152 \\
\hline PCS_w2 & $<-$ & Level & -0.691 & 0.343 & -2.014 & 0.044 & -0.126 \\
\hline WIPL_w1 & $<-$ & Level & -0.117 & 0.024 & -4.793 & 0.000 & -0.288 \\
\hline PCS_w2 & $<-$ & WIPL_w1 & -1.641 & 0.972 & -1.688 & 0.091 & -0.121 \\
\hline WIPL1_w1 & $<-$ & WIPL_w1 & 1 & & & & 0.528 \\
\hline WIPL2_w1 & $<-$ & WIPL_w1 & 1.822 & 0.184 & 9.883 & 0.000 & 0.852 \\
\hline WIPL3_w1 & $<-$ & WIPL_w1 & 1.706 & 0.173 & 9.853 & 0.000 & 0.846 \\
\hline WIPL4_w1 & $<-$ & WIPL_w1 & 1.925 & 0.188 & 10.214 & 0.000 & 0.926 \\
\hline WIPL5_w1 & $<-$ & WIPL_w1 & 1.584 & 0.171 & 9.270 & 0.000 & 0.748 \\
\hline
\end{tabular}




\section{Model 4. WIPL, WtN Beh, MCS}

Work-to-nonwork conflict, work-to-nonwork behavior-based enhancement, and mental health

\section{Unstandardized Output:}

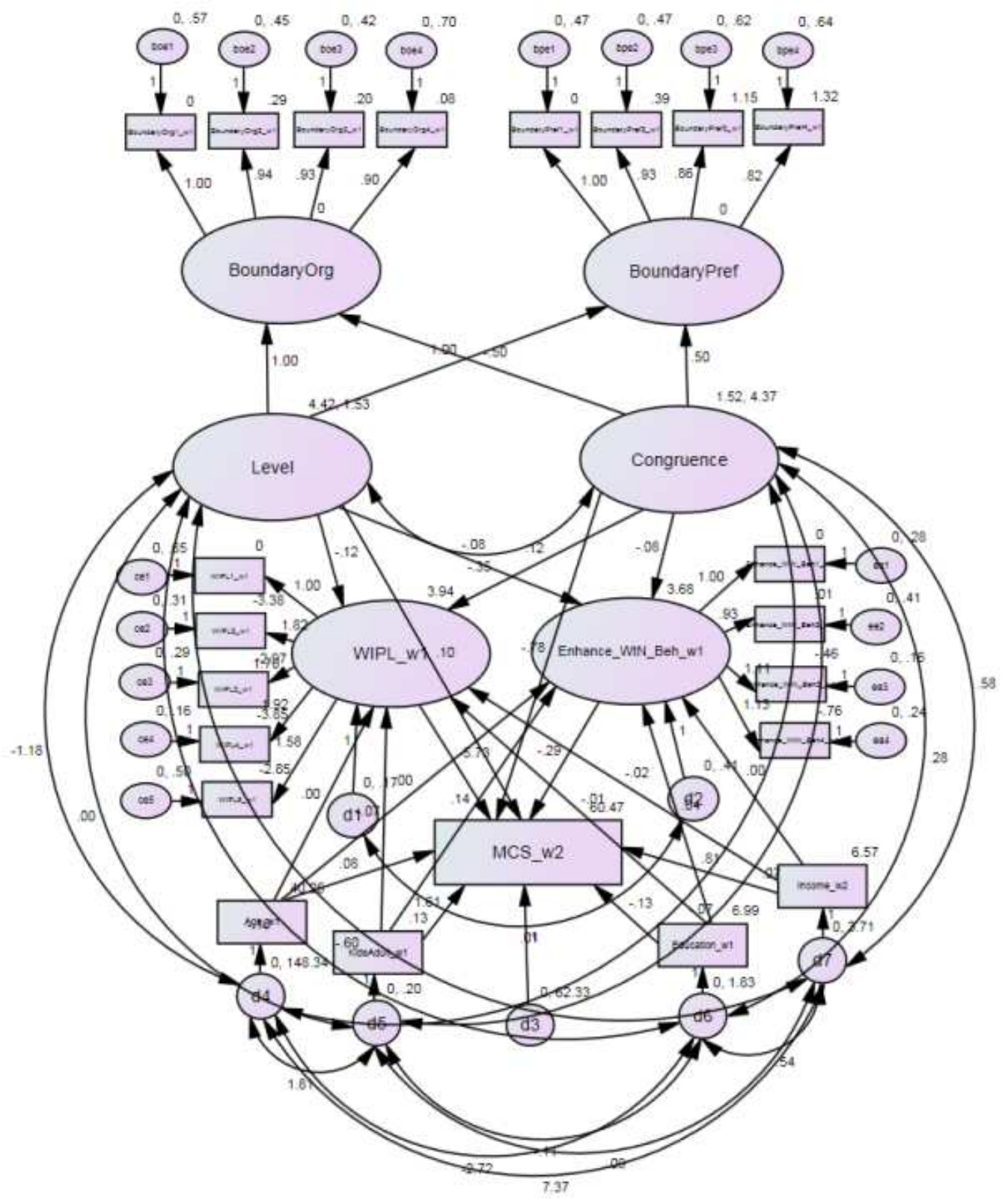

Fit Statistics:

- $\chi^{2}(178)=264.928, p<.001$

- CFI: 980

- RMSEA: .039 
Model 4 Output

\begin{tabular}{|c|c|c|c|c|c|c|c|}
\hline & & & Est. & S.E. & C.R. & $\mathrm{P}$ & $\begin{array}{l}\text { Std. } \\
\text { Est. }\end{array}$ \\
\hline Enhance_WtN_Beh_w1 & $<-$ & Age_w1 & 0.004 & 0.004 & 1.012 & 0.311 & 0.067 \\
\hline MCS_w2 & $<-$ & Age_w1 & 0.083 & 0.043 & 1.956 & 0.050 & 0.111 \\
\hline WIPL_w1 & $<-$ & Age_w1 & 0.001 & 0.002 & 0.332 & 0.740 & 0.019 \\
\hline BoundaryOrg1_w1 & $<-$ & BoundaryOrg & 1 & & & & 0.916 \\
\hline BoundaryOrg2_w1 & $<-$ & BoundaryOrg & 0.944 & 0.034 & 28.065 & 0.000 & 0.924 \\
\hline BoundaryOrg3_w1 & $<-$ & BoundaryOrg & 0.930 & 0.033 & 28.341 & 0.000 & 0.928 \\
\hline BoundaryOrg4_w1 & $<-$ & BoundaryOrg & 0.896 & 0.037 & 24.428 & 0.000 & 0.879 \\
\hline BoundaryPref1_w1 & $<-$ & BoundaryPref & 1 & & & & 0.910 \\
\hline BoundaryPref2_w1 & $<-$ & BoundaryPref & 0.934 & 0.038 & 24.304 & 0.000 & 0.899 \\
\hline BoundaryPref3_w1 & $<-$ & BoundaryPref & 0.857 & 0.039 & 21.727 & 0.000 & 0.854 \\
\hline BoundaryPref4_w1 & $<-$ & BoundaryPref & 0.822 & 0.039 & 20.966 & 0.000 & 0.840 \\
\hline BoundaryOrg & $<-$ & Congruence & -0.500 & & & & -0.607 \\
\hline BoundaryPref & $<-$ & Congruence & 0.500 & & & & 0.693 \\
\hline Enhance_WtN_Beh_w1 & $<-$ & Congruence & -0.075 & 0.020 & -3.770 & 0.000 & -0.234 \\
\hline MCS_w2 & $<-$ & Congruence & -0.779 & 0.278 & -2.803 & 0.005 & -0.179 \\
\hline WIPL_w1 & $<-$ & Congruence & 0.116 & 0.017 & 6.930 & 0.000 & 0.481 \\
\hline Enhance_WtN_Beh_w1 & $<-$ & Education_w1 & 0.042 & 0.031 & 1.379 & 0.168 & 0.085 \\
\hline MCS_w2 & $<-$ & Education_w1 & -0.129 & 0.357 & -0.360 & 0.718 & -0.019 \\
\hline WIPL_w1 & $<-$ & Education_w1 & -0.009 & 0.020 & -0.476 & 0.634 & -0.025 \\
\hline Enhance_WtN_Beh1_w1 & $<-$ & Enhance_WtN_Beh_w1 & 1 & & & & 0.787 \\
\hline Enhance_WtN_Beh2_w1 & $<-$ & Enhance_WtN_Beh_w1 & 0.928 & 0.073 & 12.700 & 0.000 & 0.697 \\
\hline Enhance_WtN_Beh3_w1 & $<-$ & Enhance_WtN_Beh_w1 & 1.113 & 0.067 & 16.531 & 0.000 & 0.883 \\
\hline Enhance_WtN_Beh4_w1 & $<-$ & Enhance_WtN_Beh_w1 & 1.127 & 0.071 & 15.785 & 0.000 & 0.839 \\
\hline MCS_w2 & $<-$ & Enhance_WtN_Beh_w1 & -0.288 & 0.747 & -0.385 & 0.700 & -0.021 \\
\hline Enhance_WtN_Beh_w1 & $<-$ & Income_w2 & 0.005 & 0.023 & 0.218 & 0.827 & 0.014 \\
\hline MCS_w2 & $<-$ & Income_w2 & 0.813 & 0.268 & 3.031 & 0.002 & 0.172 \\
\hline WIPL_w1 & $<-$ & Income_w2 & -0.024 & 0.015 & -1.589 & 0.112 & -0.091 \\
\hline Enhance_WtN_Beh_w1 & $<-$ & KidsAdult_w1 & 0.139 & 0.097 & 1.433 & 0.152 & 0.091 \\
\hline MCS_w2 & $<-$ & KidsAdult_w1 & 1.614 & 1.132 & 1.425 & 0.154 & 0.078 \\
\hline WIPL_w1 & $<-$ & KidsAdult_w1 & -0.068 & 0.063 & -1.085 & 0.278 & -0.060 \\
\hline BoundaryOrg & $<-$ & Level & 1 & & & & 0.717 \\
\hline BoundaryPref & $<-$ & Level & 1 & & & & 0.820 \\
\hline Enhance_WtN_Beh_w1 & $<-$ & Level & -0.083 & 0.034 & -2.475 & 0.013 & -0.153 \\
\hline MCS_w2 & $<-$ & Level & 0.103 & 0.418 & 0.246 & 0.806 & 0.014 \\
\hline WIPL_w1 & $<-$ & Level & -0.117 & 0.024 & -4.787 & 0.000 & -0.287 \\
\hline MCS_w2 & $<-$ & WIPL_w1 & -5.734 & 1.284 & -4.467 & 0.000 & -0.318 \\
\hline WIPL1_w1 & $<-$ & WIPL_w1 & 1 & & & & 0.529 \\
\hline WIPL2_w1 & $<-$ & WIPL_w1 & 1.824 & 0.184 & 9.913 & 0.000 & 0.854 \\
\hline WIPL3_w1 & $<-$ & WIPL_w1 & 1.704 & 0.173 & 9.874 & 0.000 & 0.847 \\
\hline WIPL4_w1 & $<-$ & WIPL_w1 & 1.916 & 0.187 & 10.226 & 0.000 & 0.924 \\
\hline WIPL5_w1 & $<--$ & WIPL_w1 & 1.584 & 0.171 & 9.293 & 0.000 & 0.750 \\
\hline
\end{tabular}




\section{Model 5. WIPL, WtN Aff, MCS}

Work-to-nonwork conflict, work-to-nonwork affective enhancement, and mental health

\section{Unstandardized Output:}

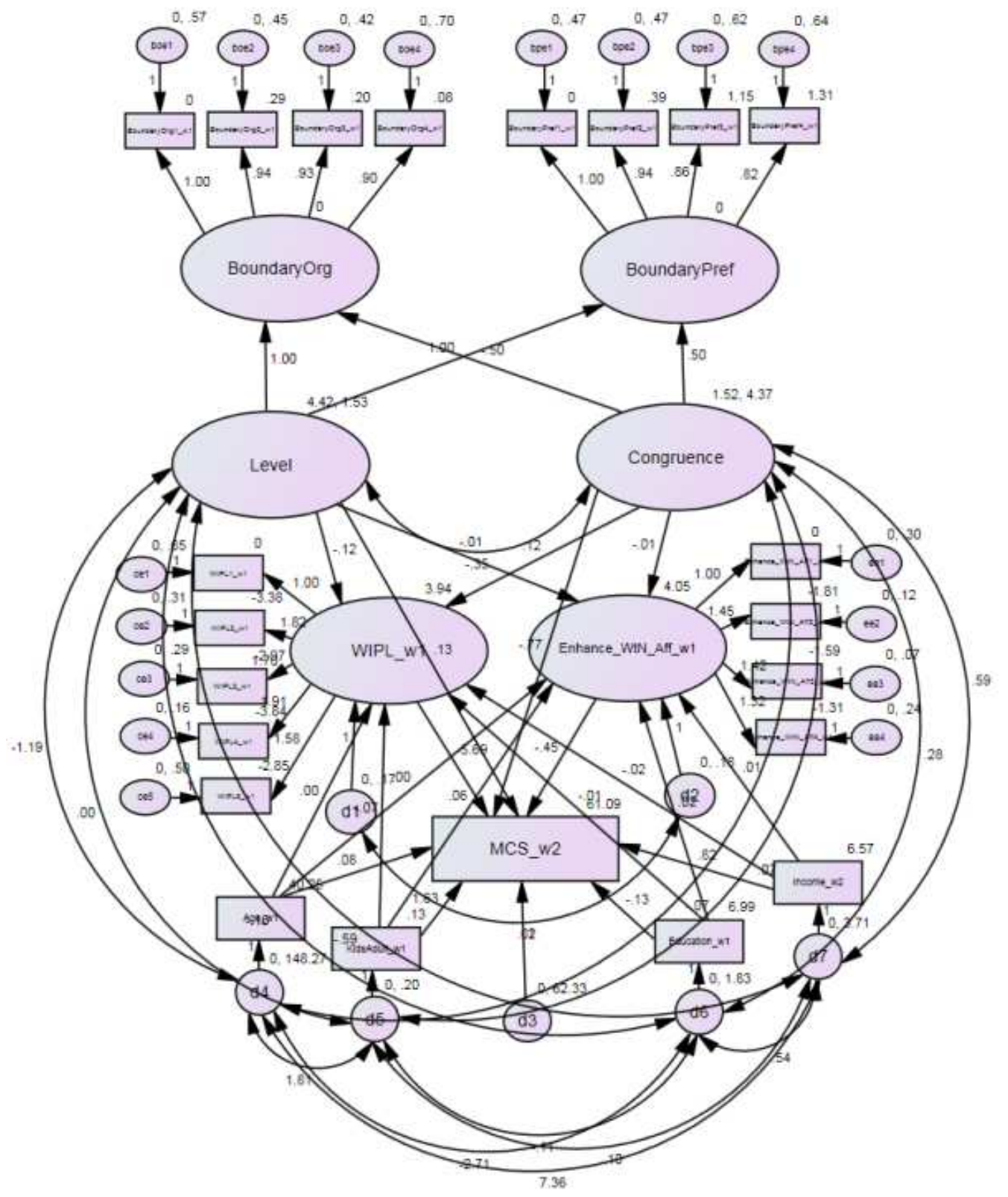

Fit Statistics:

- $\chi^{2}(178)=277.687, p<.001$

- CFI: 977

- RMSEA: .042 
Model 5 Output

\begin{tabular}{|c|c|c|c|c|c|c|c|}
\hline & & & Est. & S.E. & C.R. & $\mathrm{p}$ & $\begin{array}{l}\text { Std. } \\
\text { Est. }\end{array}$ \\
\hline Enhance_WtN_Aff_w1 & $<-$ & Age_w1 & -0.002 & 0.002 & -0.653 & 0.514 & -0.044 \\
\hline MCS_w2 & $<--$ & Age_w1 & 0.080 & 0.042 & 1.889 & 0.059 & 0.107 \\
\hline WIPL_w1 & $<--$ & Age_w1 & 0.001 & 0.002 & 0.319 & 0.750 & 0.018 \\
\hline BoundaryOrg1_w1 & $<--$ & BoundaryOrg & 1 & & & & 0.916 \\
\hline BoundaryOrg2_w1 & $<--$ & BoundaryOrg & 0.944 & 0.034 & 28.057 & 0.000 & 0.924 \\
\hline BoundaryOrg3_w1 & $<--$ & BoundaryOrg & 0.930 & 0.033 & 28.329 & 0.000 & 0.928 \\
\hline BoundaryOrg4_w1 & $<--$ & BoundaryOrg & 0.896 & 0.037 & 24.442 & 0.000 & 0.879 \\
\hline BoundaryPref1_w1 & $<--$ & BoundaryPref & 1 & & & & 0.909 \\
\hline BoundaryPref2_w1 & $<--$ & BoundaryPref & 0.935 & 0.039 & 24.269 & 0.000 & 0.899 \\
\hline BoundaryPref3_w1 & $<--$ & BoundaryPref & 0.857 & 0.040 & 21.677 & 0.000 & 0.854 \\
\hline BoundaryPref4_w1 & $<--$ & BoundaryPref & 0.823 & 0.039 & 20.964 & 0.000 & 0.841 \\
\hline BoundaryOrg & $<--$ & Congruence & -0.500 & & & & -0.607 \\
\hline BoundaryPref & $<--$ & Congruence & 0.500 & & & & 0.694 \\
\hline Enhance_WtN_Aff_w1 & $<--$ & Congruence & -0.011 & 0.013 & -0.834 & 0.404 & -0.052 \\
\hline MCS_w2 & $<-$ & Congruence & -0.768 & 0.272 & -2.829 & 0.005 & -0.177 \\
\hline WIPL_w1 & $<--$ & Congruence & 0.116 & 0.017 & 6.932 & 0.000 & 0.482 \\
\hline Enhance_WtN_Aff_w1 & $<--$ & Education_w1 & 0.016 & 0.020 & 0.816 & 0.415 & 0.052 \\
\hline MCS_w2 & $<--$ & Education_w1 & -0.134 & 0.356 & -0.377 & 0.706 & -0.02 \\
\hline WIPL_w1 & $<--$ & Education_w1 & -0.010 & 0.020 & -0.487 & 0.626 & -0.026 \\
\hline Enhance_WtN_Aff1_w1 & $<--$ & Enhance_WtN_Aff_w1 & 1 & & & & 0.608 \\
\hline Enhance_WtN_Aff2_w1 & $<--$ & Enhance_WtN_Aff_w1 & 1.449 & 0.124 & 11.662 & 0.000 & 0.871 \\
\hline Enhance_WtN_Aff3_w1 & $<--$ & Enhance_WtN_Aff_w1 & 1.422 & 0.120 & 11.856 & 0.000 & 0.912 \\
\hline Enhance_WtN_Aff4_w1 & $<--$ & Enhance_WtN_Aff_w1 & 1.317 & 0.124 & 10.643 & 0.000 & 0.754 \\
\hline MCS_w2 & $<--$ & Enhance_WtN_Aff_w1 & -0.448 & 1.134 & -0.395 & 0.692 & -0.021 \\
\hline Enhance_WtN_Aff_w1 & $<--$ & Income_w2 & 0.011 & 0.015 & 0.731 & 0.465 & 0.049 \\
\hline MCS_w2 & $<--$ & Income_w2 & 0.819 & 0.268 & 3.052 & 0.002 & 0.174 \\
\hline WIPL_w1 & $<--$ & Income_w2 & -0.024 & 0.015 & -1.577 & 0.115 & -0.09 \\
\hline Enhance_WtN_Aff_w1 & $<--$ & KidsAdult_w1 & 0.060 & 0.063 & 0.963 & 0.335 & 0.063 \\
\hline MCS_w2 & $<--$ & KidsAdult_w1 & 1.632 & 1.129 & 1.445 & 0.149 & 0.079 \\
\hline WIPL_w1 & $<--$ & KidsAdult_w1 & -0.070 & 0.063 & -1.112 & 0.266 & -0.061 \\
\hline BoundaryOrg & $<--$ & Level & 1 & & & & 0.717 \\
\hline BoundaryPref & $<--$ & Level & 1 & & & & 0.82 \\
\hline Enhance_WtN_Aff_w1 & $<--$ & Level & -0.009 & 0.022 & -0.420 & 0.675 & -0.026 \\
\hline MCS_w2 & $<-$ & Level & 0.128 & 0.414 & 0.309 & 0.757 & 0.017 \\
\hline WIPL_w1 & $<--$ & Level & -0.117 & 0.024 & -4.784 & 0.000 & -0.287 \\
\hline MCS_w2 & $<--$ & WIPL_w1 & -5.690 & 1.289 & -4.416 & 0.000 & -0.316 \\
\hline WIPL1_w1 & $<--$ & WIPL_w1 & 1 & & & & 0.529 \\
\hline WIPL2_w1 & $<--$ & WIPL_w1 & 1.823 & 0.184 & 9.922 & 0.000 & 0.854 \\
\hline WIPL3_w1 & $<--$ & WIPL_w1 & 1.702 & 0.172 & 9.880 & 0.000 & 0.846 \\
\hline WIPL4_w1 & $<--$ & WIPL_w1 & 1.915 & 0.187 & 10.236 & 0.000 & 0.924 \\
\hline WIPL5_w1 & $<--$ & WIPL_w1 & 1.584 & 0.170 & 9.300 & 0.000 & 0.750 \\
\hline
\end{tabular}




\section{Model 6. WIPL, WtN Aff, PCS}

Work-to-nonwork conflict, work-to-nonwork affective enhancement, and physical health

\section{Unstandardized Output:}

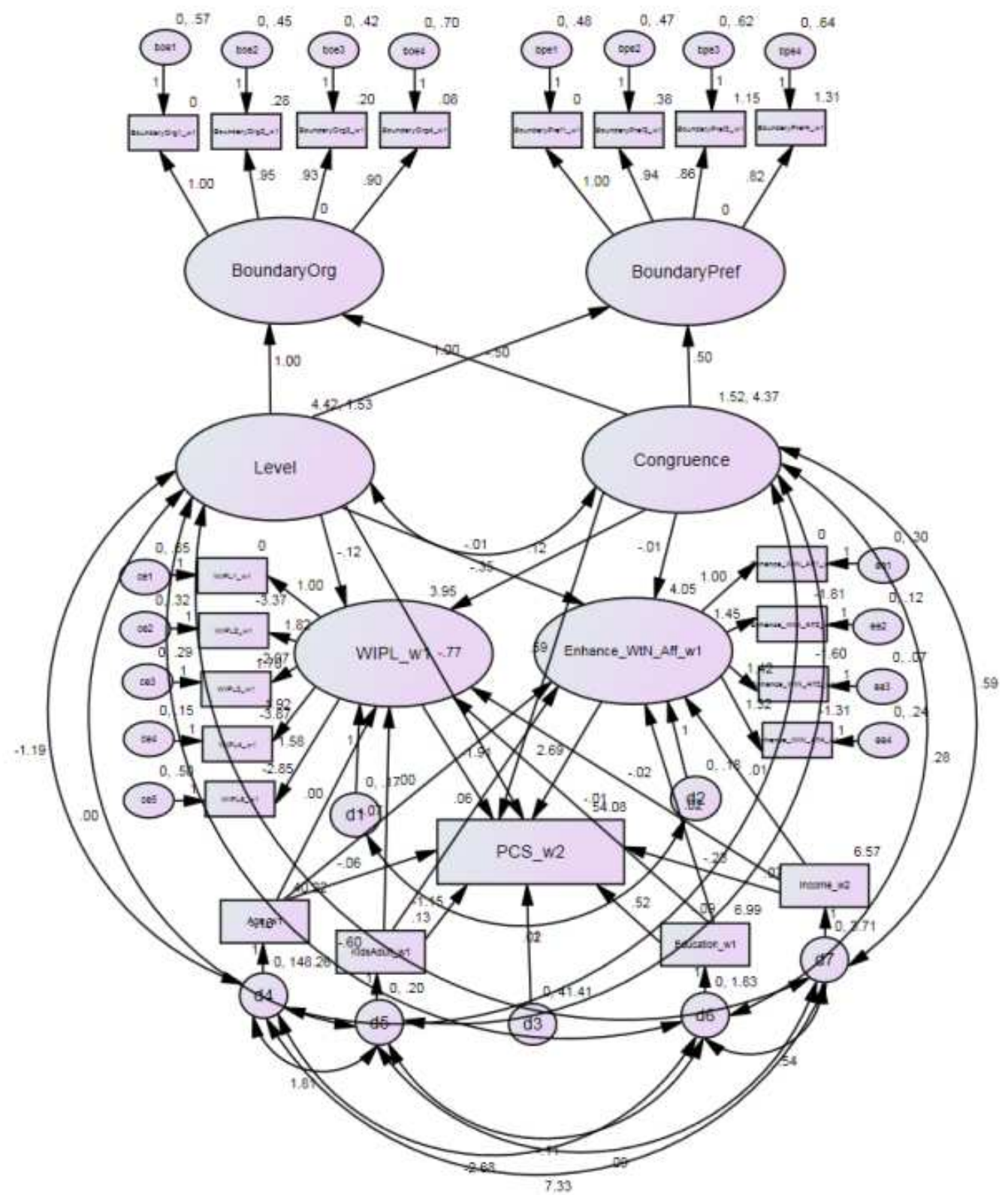

Fit Statistics:

- $\chi^{2}(178)=284.069, p<.001$

- CFI: 975

- RMSEA: .044 


\begin{tabular}{|c|c|c|c|c|c|c|c|}
\hline & & & Est. & S.E. & C.R. & $\mathrm{p}$ & Std. Est. \\
\hline Enhance_WtN_Aff_w1 & $<-$ & Age_w1 & -0.002 & 0.002 & -0.669 & 0.503 & -0.045 \\
\hline PCS_w2 & $<--$ & Age_w1 & -0.058 & 0.034 & -1.668 & 0.095 & -0.103 \\
\hline WIPL_w1 & $<--$ & Age_w1 & 0.001 & 0.002 & 0.305 & 0.761 & 0.017 \\
\hline BoundaryOrg1_w1 & $<--$ & BoundaryOrg & 1 & & & & 0.915 \\
\hline BoundaryOrg2_w1 & $<--$ & BoundaryOrg & 0.945 & 0.034 & 28.060 & 0.000 & 0.925 \\
\hline BoundaryOrg3_w1 & $<--$ & BoundaryOrg & 0.903 & 0.033 & 28.314 & 0.000 & 0.928 \\
\hline BoundaryOrg4_w1 & $<--$ & BoundaryOrg & 0.896 & 0.037 & 24.417 & 0.000 & 0.879 \\
\hline BoundaryPref1_w1 & $<--$ & BoundaryPref & 1 & & & & 0.909 \\
\hline BoundaryPref2_w1 & $<--$ & BoundaryPref & 0.936 & 0.039 & 24.269 & 0.000 & 0.9 \\
\hline BoundaryPref3_w1 & $<--$ & BoundaryPref & 0.857 & 0.040 & 21.655 & 0.000 & 0.854 \\
\hline BoundaryPref4_w1 & $<--$ & BoundaryPref & 0.824 & 0.039 & 20.957 & 0.000 & 0.841 \\
\hline BoundaryOrg & $<--$ & Congruence & -0.500 & & & & -0.607 \\
\hline BoundaryPref & $<--$ & Congruence & 0.500 & & & & 0.694 \\
\hline Enhance_WtN_Aff_w1 & $<--$ & Congruence & -0.011 & 0.013 & -0.843 & 0.399 & -0.053 \\
\hline PCS_w2 & $<--$ & Congruence & 0.594 & 0.221 & 2.690 & 0.007 & 0.183 \\
\hline WIPL_w1 & $<--$ & Congruence & 0.116 & 0.017 & 6.927 & 0.000 & 0.481 \\
\hline Enhance_WtN_Aff_w1 & $<--$ & Education_w1 & 0.016 & 0.020 & 0.819 & 0.413 & 0.052 \\
\hline PCS_w2 & $<--$ & Education_w1 & 0.521 & 0.289 & 1.801 & 0.072 & 0.104 \\
\hline WIPL_w1 & $<--$ & Education_w1 & -0.010 & 0.020 & -0.497 & 0.619 & -0.026 \\
\hline Enhance_WtN_Aff1_w1 & $<--$ & Enhance_WtN_Aff_w1 & 1 & & & & 0.608 \\
\hline Enhance_WtN_Aff2_w1 & $<--$ & Enhance_WtN_Aff_w1 & 1.449 & 0.124 & 11.66 & 0.000 & 0.872 \\
\hline Enhance_WtN_Aff3_w1 & $<--$ & Enhance_WtN_Aff_w1 & 1.422 & 0.120 & 11.857 & 0.000 & 0.912 \\
\hline Enhance_WtN_Aff4_w1 & $<--$ & Enhance_WtN_Aff_w1 & 1.318 & 0.124 & 10.642 & 0.000 & 0.754 \\
\hline PCS_w2 & $<--$ & Enhance_WtN_Aff_w1 & 2.688 & 0.944 & 2.848 & 0.004 & 0.167 \\
\hline Enhance_WtN_Aff_w1 & $<--$ & Income_w2 & 0.011 & 0.015 & 0.766 & 0.444 & 0.052 \\
\hline PCS_w2 & $<--$ & Income_w2 & -0.225 & 0.218 & -1.032 & 0.302 & -0.064 \\
\hline WIPL_w1 & $<--$ & Income_w2 & -0.024 & 0.015 & -1.587 & 0.113 & -0.091 \\
\hline Enhance_WtN_Aff_w1 & $<--$ & KidsAdult_w1 & 0.061 & 0.062 & 0.979 & 0.328 & 0.064 \\
\hline PCS_w2 & $<--$ & KidsAdult_w1 & -1.152 & 0.918 & -1.255 & 0.210 & -0.075 \\
\hline WIPL_w1 & $<--$ & KidsAdult_w1 & -0.070 & 0.063 & -1.113 & 0.266 & -0.061 \\
\hline BoundaryOrg & $<--$ & Level & 1 & & & & 0.717 \\
\hline BoundaryPref & $<--$ & Level & 1 & & & & 0.82 \\
\hline Enhance_WtN_Aff_w1 & $<-$ & Level & -0.009 & 0.022 & -0.399 & 0.690 & -0.025 \\
\hline PCS_w2 & $<--$ & Level & -0.772 & 0.337 & -2.292 & 0.022 & -0.14 \\
\hline WIPL_w1 & $<--$ & Level & -0.117 & 0.024 & -4.790 & 0.000 & -0.288 \\
\hline PCS_w2 & $<--$ & WIPL_w1 & -1.905 & 0.973 & -1.958 & 0.050 & -0.141 \\
\hline WIPL1_w1 & $<-$ & WIPL_w1 & 1 & & & & 0.529 \\
\hline WIPL2_w1 & $<--$ & WIPL_w1 & 1.821 & 0.184 & 9.894 & 0.000 & 0.852 \\
\hline WIPL3_w1 & $<--$ & WIPL_w1 & 1.703 & 0.173 & 9.859 & 0.000 & 0.845 \\
\hline WIPL4_w1 & $<--$ & WIPL_w1 & 1.923 & 0.188 & 10.226 & 0.000 & 0.926 \\
\hline WIPL5_w1 & $<--$ & WIPL_w1 & 1.583 & 0.171 & 9.278 & 0.000 & 0.748 \\
\hline
\end{tabular}




\section{Model 7. PLIW, WtN Aff, PCS}

Nonwork-to-work conflict, work-to-nonwork affective enhancement, and physical health

\section{Unstandardized Output:}

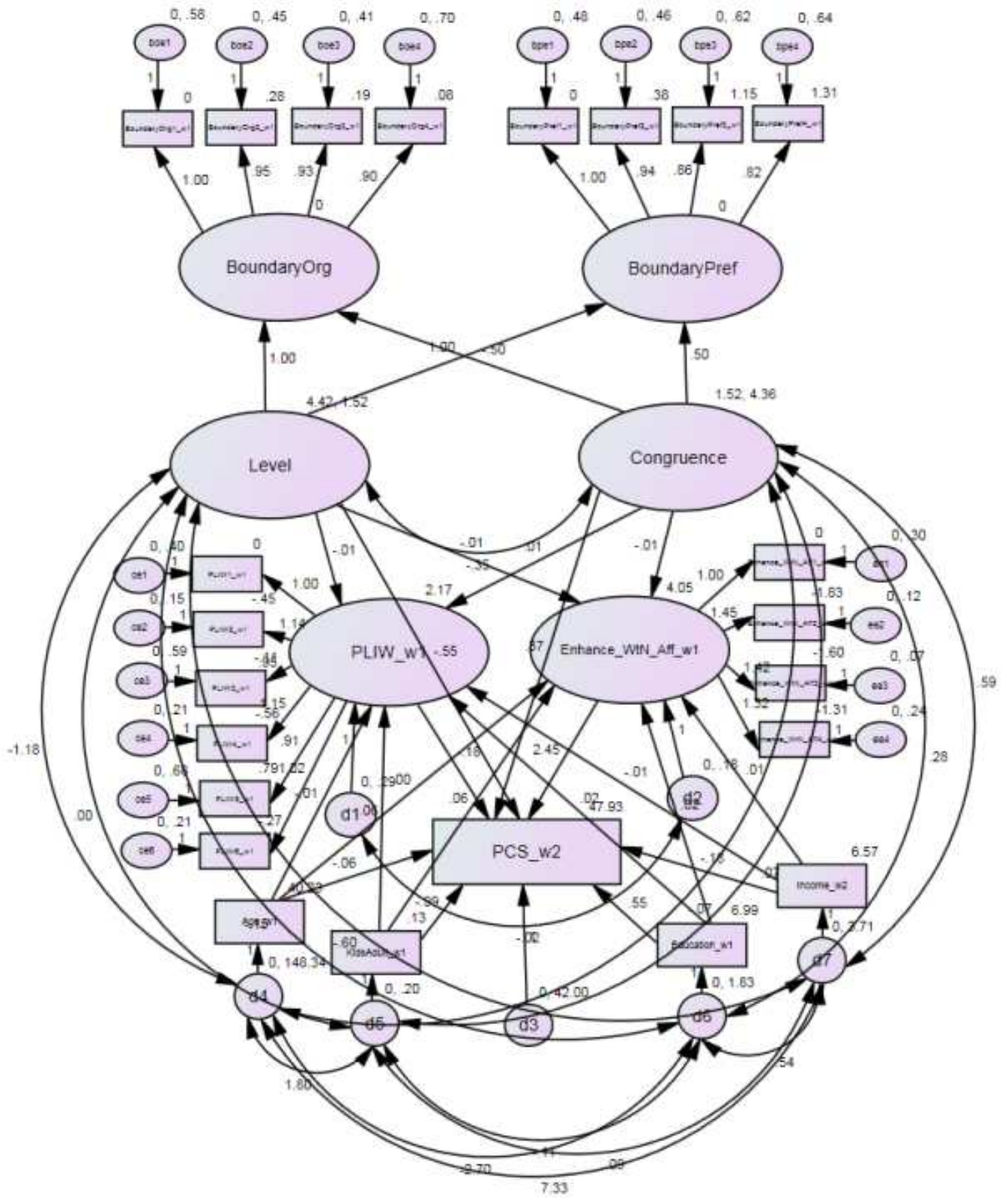

Fit Statistics:

- $\chi^{2}(199)=310.621, p<.001$

- $\quad$ CFI: .971

- RMSEA: .042 
Model 7 Output

\begin{tabular}{|c|c|c|c|c|c|c|c|}
\hline & & & Est. & S.E. & C.R. & $\mathrm{p}$ & $\begin{array}{l}\text { Std. } \\
\text { Est. }\end{array}$ \\
\hline Enhance_WtN_Aff_w1 & $<--$ & Age_w1 & -0.002 & 0.002 & -0.659 & 0.510 & -0.045 \\
\hline PCS_w2 & $<--$ & Age_w1 & -0.061 & 0.035 & -1.736 & 0.083 & -0.109 \\
\hline PLIW_w1 & $<--$ & Age_w1 & -0.006 & 0.003 & -1.945 & 0.052 & -0.134 \\
\hline BoundaryOrg1_w1 & $<--$ & BoundaryOrg & 1 & & & & 0.914 \\
\hline BoundaryOrg2_w1 & $<--$ & BoundaryOrg & 0.946 & 0.034 & 27.876 & 0.000 & 0.925 \\
\hline BoundaryOrg3_w1 & $<--$ & BoundaryOrg & 0.932 & 0.033 & 28.212 & 0.000 & 0.929 \\
\hline BoundaryOrg4_w1 & $<--$ & BoundaryOrg & 0.898 & 0.037 & 24.319 & 0.000 & 0.879 \\
\hline BoundaryPref1_w1 & $<--$ & BoundaryPref & 1 & & & & 0.909 \\
\hline BoundaryPref2_w1 & $<--$ & BoundaryPref & 0.937 & 0.039 & 24.287 & 0.000 & 0.900 \\
\hline BoundaryPref3_w1 & $<--$ & BoundaryPref & 0.857 & 0.040 & 21.620 & 0.000 & 0.854 \\
\hline BoundaryPref4_w1 & $<--$ & BoundaryPref & 0.824 & 0.039 & 20.914 & 0.000 & 0.840 \\
\hline BoundaryOrg & $<--$ & Congruence & -0.500 & & & & -0.607 \\
\hline BoundaryPref & $<--$ & Congruence & 0.500 & & & & 0.693 \\
\hline Enhance_WtN_Aff_w1 & $<--$ & Congruence & -0.011 & 0.013 & -0.846 & 0.398 & -0.053 \\
\hline PCS_w2 & $<--$ & Congruence & 0.373 & 0.187 & 1.995 & 0.046 & 0.115 \\
\hline PLIW_w1 & $<--$ & Congruence & 0.013 & 0.016 & 0.787 & 0.432 & 0.050 \\
\hline Enhance_WtN_Aff_w1 & $<--$ & Education_w1 & 0.016 & 0.020 & 0.819 & 0.413 & 0.052 \\
\hline PCS_w2 & $<--$ & Education_w1 & 0.546 & 0.291 & 1.878 & 0.060 & 0.109 \\
\hline PLIW_w1 & $<--$ & Education_w1 & 0.020 & 0.026 & 0.798 & 0.425 & 0.051 \\
\hline Enhance_WtN_Aff1_w1 & $<--$ & Enhance_WtN_Aff_w1 & 1 & & & & 0.607 \\
\hline Enhance_WtN_Aff2_w1 & $<--$ & Enhance_WtN_Aff_w1 & 1.454 & 0.125 & 11.645 & 0.000 & 0.873 \\
\hline Enhance_WtN_Aff3_w1 & $<--$ & Enhance_WtN_Aff_w1 & 1.424 & 0.120 & 11.831 & 0.000 & 0.912 \\
\hline Enhance_WtN_Aff4_w1 & $<--$ & Enhance_WtN_Aff_w1 & 1.318 & 0.124 & 10.612 & 0.000 & 0.753 \\
\hline PCS_w2 & $<--$ & Enhance_WtN_Aff_w1 & 2.452 & 0.943 & 2.602 & 0.009 & 0.152 \\
\hline Enhance_WtN_Aff_w1 & $<--$ & Income_w2 & 0.011 & 0.015 & 0.760 & 0.447 & 0.051 \\
\hline PCS_w2 & $<--$ & Income_w2 & -0.178 & 0.218 & -0.816 & 0.415 & -0.050 \\
\hline PLIW_w1 & $<--$ & Income_w2 & -0.006 & 0.019 & -0.292 & 0.770 & -0.020 \\
\hline Enhance_WtN_Aff_w1 & $<--$ & KidsAdult_w1 & 0.061 & 0.062 & 0.973 & 0.331 & 0.064 \\
\hline PCS_w2 & $<--$ & KidsAdult_w1 & -0.994 & 0.921 & -1.080 & 0.280 & -0.065 \\
\hline PLIW_w1 & $<--$ & KidsAdult_w1 & 0.055 & 0.081 & 0.683 & 0.495 & 0.045 \\
\hline BoundaryOrg & $<--$ & Level & 1 & & & & 0.717 \\
\hline BoundaryPref & $<--$ & Level & 1 & & & & 0.820 \\
\hline Enhance_WtN_Aff_w1 & $<--$ & Level & -0.009 & 0.022 & -0.396 & 0.692 & -0.025 \\
\hline PCS_w2 & $<--$ & Level & -0.553 & 0.318 & -1.739 & 0.082 & -0.100 \\
\hline PLIW_w1 & $<--$ & Level & -0.011 & 0.028 & -0.377 & 0.706 & -0.024 \\
\hline PCS_w2 & $<--$ & PLIW_w1 & -0.184 & 0.735 & -0.250 & 0.802 & -0.015 \\
\hline PLIW1_w1 & $<--$ & PLIW_w1 & 1 & & & & 0.649 \\
\hline PLIW2_w1 & $<--$ & PLIW_w1 & 1.138 & 0.094 & 12.058 & 0.000 & 0.844 \\
\hline PLIW3_w1 & $<--$ & PLIW_w1 & 0.952 & 0.110 & 8.668 & 0.000 & 0.557 \\
\hline PLIW4_w1 & $<--$ & PLIW_w1 & 1.153 & 0.099 & 11.693 & 0.000 & 0.804 \\
\hline PLIW5_w1 & $<--$ & PLIW_w1 & 0.915 & 0.113 & 8.111 & 0.000 & 0.516 \\
\hline PLIW6_w1 & $<--$ & PLIW_w1 & 1.025 & 0.090 & 11.328 & 0.000 & 0.771 \\
\hline
\end{tabular}




\section{Model 8. PLIW, WtN Aff, MCS}

Nonwork-to-work conflict, work-to-nonwork affective enhancement, and mental health

\section{Unstandardized Output:}

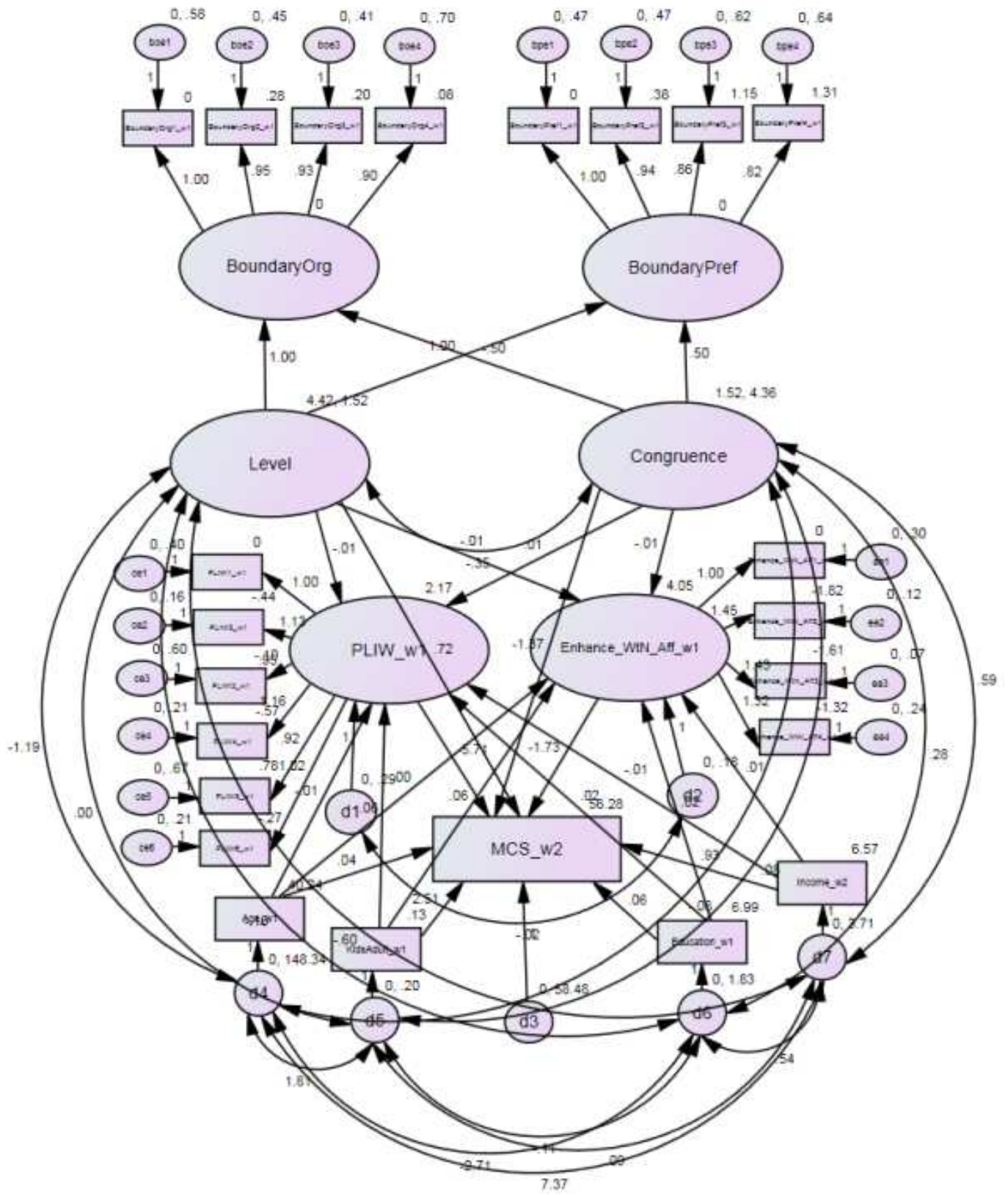

Fit Statistics:

- $\chi^{2}(199)=302.975, p<.001$

- CFI: .974

- RMSEA: .041 
Model 8 Output

\begin{tabular}{|c|c|c|c|c|c|c|c|}
\hline & & & Est. & S.E. & C.R. & $\mathrm{P}$ & Std. Est. \\
\hline Enhance_WtN_Aff_w1 & $<-$ & Age_w1 & -0.002 & 0.002 & -0.652 & 0.514 & -0.044 \\
\hline MCS_w2 & $<-$ & Age_w1 & 0.038 & 0.042 & 0.918 & 0.358 & 0.052 \\
\hline PLIW_w1 & $<-$ & Age_w1 & -0.006 & 0.003 & -1.967 & 0.049 & -0.136 \\
\hline BoundaryOrg1_w1 & $<-$ & BoundaryOrg & 1 & & & & 0.915 \\
\hline BoundaryOrg2_w1 & $<-$ & BoundaryOrg & 0.946 & 0.034 & 27.970 & 0.000 & 0.925 \\
\hline BoundaryOrg3_w1 & $<-$ & BoundaryOrg & 0.931 & 0.033 & 28.262 & 0.000 & 0.928 \\
\hline BoundaryOrg4_w1 & $<-$ & BoundaryOrg & 0.897 & 0.037 & 24.344 & 0.000 & 0.879 \\
\hline BoundaryPref1_w1 & $<--$ & BoundaryPref & 1 & & & & 0.909 \\
\hline BoundaryPref2_w1 & $<-$ & BoundaryPref & 0.936 & 0.039 & 24.279 & 0.000 & 0.900 \\
\hline BoundaryPref3_w1 & $<-$ & BoundaryPref & 0.857 & 0.040 & 21.659 & 0.000 & 0.854 \\
\hline BoundaryPref4_w1 & $<-$ & BoundaryPref & 0.823 & 0.039 & 20.957 & 0.000 & 0.841 \\
\hline BoundaryOrg & $<-$ & Congruence & -0.500 & & & & -0.607 \\
\hline BoundaryPref & $<-$ & Congruence & 0.500 & & & & 0.693 \\
\hline Enhance_WtN_Aff_w1 & $<-$ & Congruence & -0.011 & 0.013 & -0.838 & 0.402 & -0.053 \\
\hline MCS_w2 & $<-$ & Congruence & -1.370 & 0.225 & -6.088 & 0.000 & -0.315 \\
\hline PLIW_w1 & $<-$ & Congruence & 0.013 & 0.016 & 0.793 & 0.428 & 0.050 \\
\hline Enhance_WtN_Aff_w1 & $<-$ & Education_w1 & 0.016 & 0.020 & 0.812 & 0.417 & 0.051 \\
\hline MCS_w2 & $<-$ & Education_w1 & 0.062 & 0.348 & 0.178 & 0.859 & 0.009 \\
\hline PLIW_w1 & $<-$ & Education_w1 & 0.021 & 0.026 & 0.822 & 0.411 & 0.052 \\
\hline Enhance_WtN_Aff1_w1 & $<-$ & Enhance_WtN_Aff_w1 & 1 & & & & 0.607 \\
\hline Enhance_WtN_Aff2_w1 & $<-$ & Enhance_WtN_Aff_w1 & 1.453 & 0.125 & 11.632 & 0.000 & 0.872 \\
\hline Enhance_WtN_Aff3_w1 & $<-$ & Enhance_WtN_Aff_w1 & 1.426 & 0.121 & 11.825 & 0.000 & 0.913 \\
\hline Enhance_WtN_Aff4_w1 & $<-$ & Enhance_WtN_Aff_w1 & 1.319 & 0.124 & 10.611 & 0.000 & 0.753 \\
\hline MCS_w2 & $<-$ & Enhance_WtN_Aff_w1 & -1.734 & 1.115 & -1.556 & 0.120 & -0.080 \\
\hline Enhance_WtN_Aff_w1 & $<-$ & Income_w2 & 0.011 & 0.015 & 0.737 & 0.461 & 0.050 \\
\hline MCS_w2 & $<-$ & Income_w2 & 0.932 & 0.261 & 3.572 & 0.000 & 0.197 \\
\hline PLIW_w1 & $<-$ & Income_w2 & -0.006 & 0.019 & -0.329 & 0.742 & -0.022 \\
\hline Enhance_WtN_Aff_w1 & $<-$ & KidsAdult_w1 & 0.060 & 0.062 & 0.957 & 0.339 & 0.063 \\
\hline MCS_w2 & $<-$ & KidsAdult_w1 & 2.506 & 1.102 & 2.275 & 0.023 & 0.122 \\
\hline PLIW_w1 & $<-$ & KidsAdult_w1 & 0.059 & 0.081 & 0.735 & 0.462 & 0.048 \\
\hline BoundaryOrg & $<-$ & Level & 1 & & & & 0.717 \\
\hline BoundaryPref & $<-$ & Level & 1 & & & & 0.820 \\
\hline Enhance_WtN_Aff_w1 & $<-$ & Level & -0.009 & 0.022 & -0.418 & 0.676 & -0.026 \\
\hline MCS_w2 & $<-$ & Level & 0.722 & 0.383 & 1.885 & 0.059 & 0.098 \\
\hline PLIW_w1 & $<-$ & Level & -0.011 & 0.028 & -0.384 & 0.701 & -0.024 \\
\hline MCS_w2 & $<-$ & PLIW_w1 & -5.705 & 0.963 & -5.925 & 0.000 & -0.340 \\
\hline PLIW1_w1 & $<-$ & PLIW_w1 & 1 & & & & 0.649 \\
\hline PLIW2_w1 & $<-$ & PLIW_w1 & 1.133 & 0.094 & 12.056 & 0.000 & 0.840 \\
\hline PLIW3_w1 & $<--$ & PLIW_w1 & 0.947 & 0.110 & 8.642 & 0.000 & 0.554 \\
\hline PLIW4_w1 & $<-$ & PLIW_w1 & 1.160 & 0.099 & 11.760 & 0.000 & 0.809 \\
\hline PLIW5_w1 & & PLIW_w1 & 0.921 & 0.113 & 8.170 & 0.000 & 0.520 \\
\hline PLIW6_w1 & $<-$ & PLIW_w1 & 1.025 & 0.090 & 11.349 & 0.000 & 0.771 \\
\hline
\end{tabular}




\section{Model 9. PLIW, WtN Val, MCS}

Nonwork-to-work conflict, work-to-nonwork value-based enhancement, and mental health

\section{Unstandardized Output:}

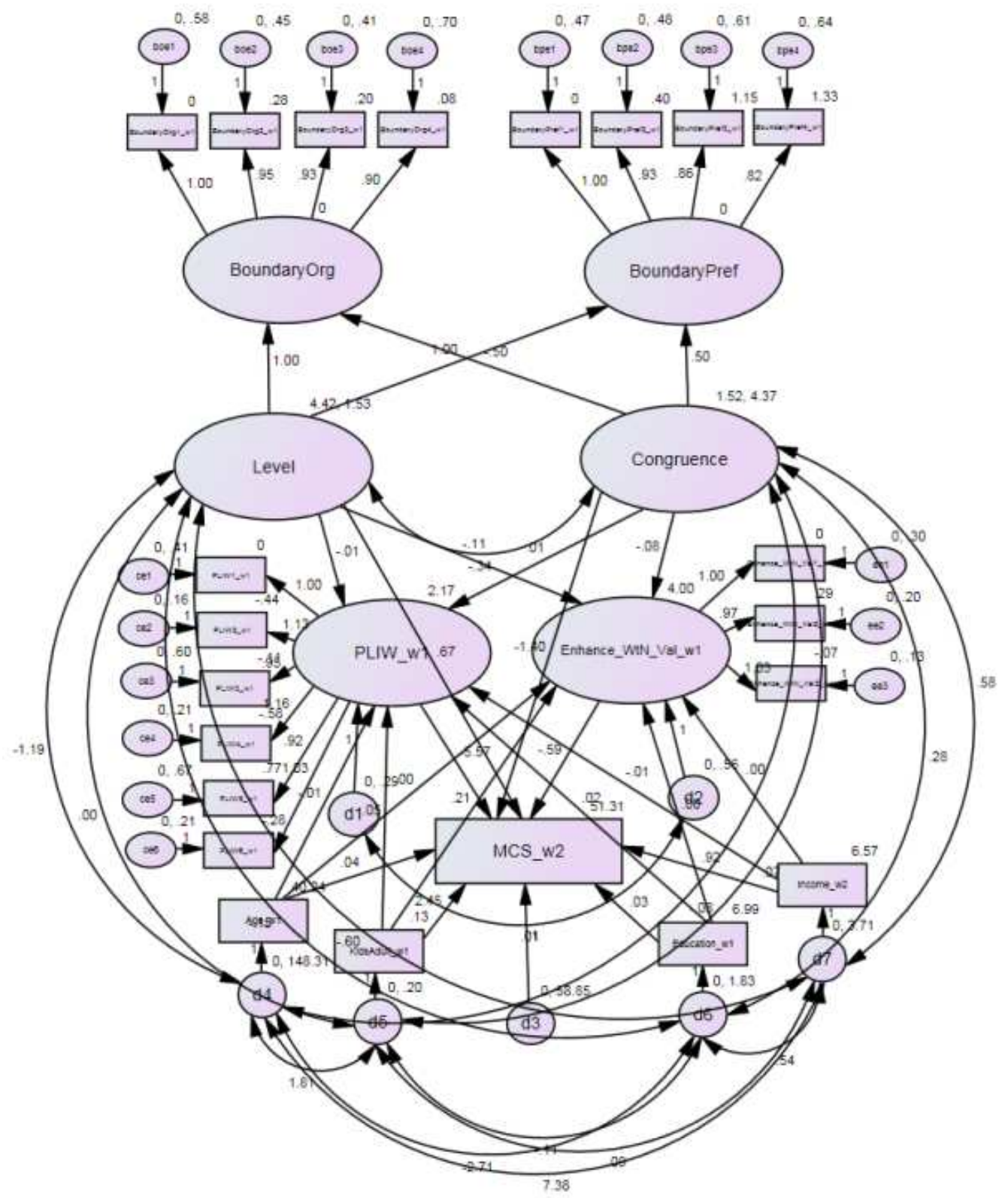

Fit Statistics:

- $\chi^{2}(178)=286.896, p<.001$

- CFI: 972

- RMSEA: .044 
Model 9 Output

\begin{tabular}{|c|c|c|c|c|c|c|c|}
\hline & & & Est. & S.E. & C.R. & $\mathrm{P}$ & Std. Est. \\
\hline Enhance_WtN_Val_w1 & $<--$ & Age_w1 & 0.001 & 0.004 & 0.330 & 0.741 & 0.022 \\
\hline MCS_w2 & $<-$ & Age_w1 & 0.043 & 0.042 & 1.034 & 0.301 & 0.058 \\
\hline PLIW_w1 & $<-$ & Age_w1 & -0.006 & 0.003 & -1.955 & 0.051 & -0.135 \\
\hline BoundaryOrg1_w1 & $<-$ & BoundaryOrg & 1 & & & & 0.915 \\
\hline BoundaryOrg2_w1 & $<-$ & BoundaryOrg & 0.946 & 0.034 & 27.966 & 0.000 & 0.925 \\
\hline BoundaryOrg3_w1 & $<-$ & BoundaryOrg & 0.931 & 0.033 & 28.268 & 0.000 & 0.928 \\
\hline BoundaryOrg4_w1 & $<-$ & BoundaryOrg & 0.897 & 0.037 & 24.338 & 0.000 & 0.879 \\
\hline BoundaryPref1_w1 & $<-$ & BoundaryPref & 1 & & & & 0.911 \\
\hline BoundaryPref2_w1 & $<-$ & BoundaryPref & 0.933 & 0.038 & 24.284 & 0.000 & 0.898 \\
\hline BoundaryPref3_w1 & $<-$ & BoundaryPref & 0.857 & 0.039 & 21.806 & 0.000 & 0.855 \\
\hline BoundaryPref4_w1 & $<-$ & BoundaryPref & 0.821 & 0.039 & 20.952 & 0.000 & 0.839 \\
\hline BoundaryOrg & $<-$ & Congruence & -0.500 & & & & -0.607 \\
\hline BoundaryPref & $<-$ & Congruence & 0.500 & & & & 0.693 \\
\hline Enhance_WtN_Val_w1 & $<-$ & Congruence & -0.079 & 0.023 & -3.447 & 0.000 & -0.212 \\
\hline MCS_w2 & $<-$ & Congruence & -1.398 & 0.231 & -6.044 & 0.000 & -0.322 \\
\hline PLIW_w1 & $<-$ & Congruence & 0.013 & 0.016 & 0.800 & 0.424 & 0.051 \\
\hline Enhance_WtN_Val_w1 & $<-$ & Education_w1 & 0 & 0.035 & 0.012 & 0.990 & 0.001 \\
\hline MCS_w2 & $<-$ & Education_w1 & 0.028 & 0.349 & 0.082 & 0.935 & 0.004 \\
\hline PLIW_w1 & $<-$ & Education_w1 & 0.021 & 0.026 & 0.815 & 0.415 & 0.052 \\
\hline Enhance_WtN_Val1_w1 & $<-$ & Enhance_WtN_Val_w1 & 1 & & & & 0.819 \\
\hline Enhance_WtN_Val2_w1 & $<-$ & Enhance_WtN_Val_w1 & 0.969 & 0.055 & 17.613 & 0.000 & 0.859 \\
\hline Enhance_WtN_Val3_w1 & $<-$ & Enhance_WtN_Val_w1 & 1.028 & 0.056 & 18.402 & 0.000 & 0.914 \\
\hline MCS_w2 & $<-$ & Enhance_WtN_Val_w1 & -0.589 & 0.622 & -0.946 & 0.344 & -0.051 \\
\hline Enhance_WtN_Val_w1 & $<-$ & Income_w2 & 0.003 & 0.027 & 0.118 & 0.906 & 0.008 \\
\hline MCS_w2 & $<--$ & Income_w2 & 0.916 & 0.261 & 3.506 & 0.000 & 0.194 \\
\hline PLIW_w1 & $<-$ & Income_w2 & -0.006 & 0.019 & -0.325 & 0.745 & -0.022 \\
\hline Enhance_WtN_Val_w1 & $<-$ & KidsAdult_w1 & 0.210 & 0.112 & 1.873 & 0.061 & 0.119 \\
\hline MCS_w 2 & $<-$ & KidsAdult_w1 & 2.447 & 1.110 & 2.204 & 0.028 & 0.119 \\
\hline PLIW_w1 & $<-$ & KidsAdult_w1 & 0.054 & 0.081 & 0.669 & 0.503 & 0.044 \\
\hline BoundaryOrg & $<-$ & Level & 1 & & & & 0.718 \\
\hline BoundaryPref & $<-$ & Level & 1 & & & & 0.819 \\
\hline Enhance_WtN_Val_w1 & $<-$ & Level & -0.115 & 0.039 & -2.940 & 0.003 & -0.181 \\
\hline MCS_w2 & $<-$ & Level & 0.672 & 0.391 & 1.718 & 0.086 & 0.091 \\
\hline PLIW_w1 & $<-$ & Level & -0.011 & 0.028 & -0.384 & 0.701 & -0.024 \\
\hline MCS_w2 & $<-$ & PLIW_w1 & -5.571 & 0.960 & -5.805 & 0.000 & -0.332 \\
\hline PLIW1_w1 & $<-$ & PLIW_w1 & 1 & & & & 0.648 \\
\hline PLIW2_w1 & $<-$ & PLIW_w1 & 1.134 & 0.094 & 12.010 & 0.000 & 0.838 \\
\hline PLIW3_w1 & $<-$ & PLIW_w1 & 0.951 & 0.110 & 8.641 & 0.000 & 0.555 \\
\hline PLIW4_w1 & $<-$ & PLIW_w1 & 1.163 & 0.099 & 11.735 & 0.000 & 0.809 \\
\hline PLIW5_w1 & $<-$ & PLIW_w1 & 0.925 & 0.113 & 8.176 & 0.000 & 0.521 \\
\hline PLIW6_w1 & $<-$ & PLIW_w1 & 1.028 & 0.091 & 11.333 & 0.000 & 0.772 \\
\hline
\end{tabular}




\section{Model 10. PLIW, WtN Val, PCS}

Nonwork-to-work conflict, work-to-nonwork value-based enhancement, and physical health

\section{Unstandardized Output:}

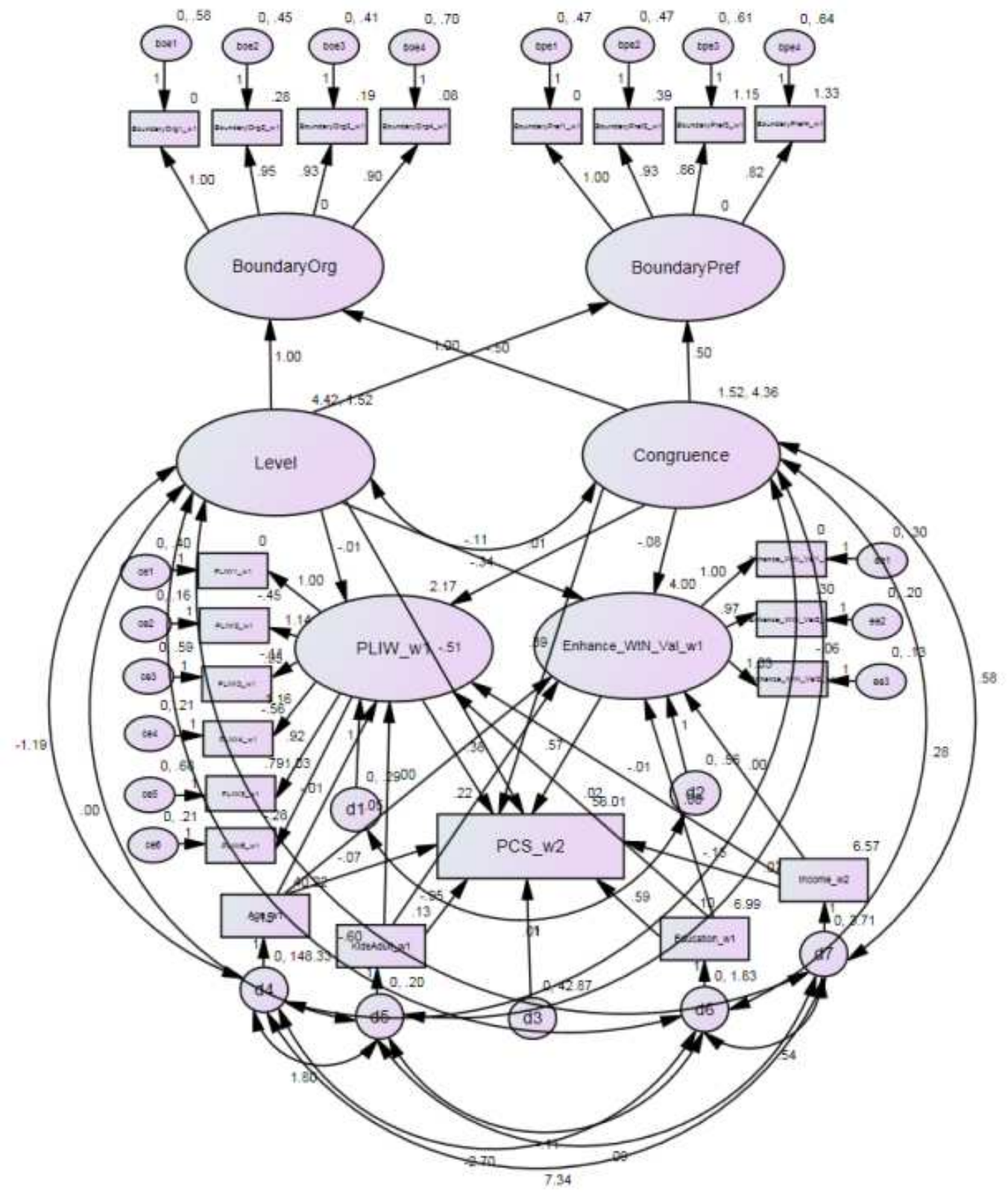

Fit Statistics:

- $\chi^{2}(178)=298.824, p<.001$

- CFI: 927

- RMSEA: .046 
$\underline{\text { Model } 10 \text { Output }}$

\begin{tabular}{|c|c|c|c|c|c|c|c|}
\hline & & & Est. & S.E. & C.R. & $\mathrm{p}$ & $\begin{array}{l}\text { Std. } \\
\text { Est. }\end{array}$ \\
\hline Enhance_WtN_Val_w1 & $<--$ & Age_w1 & 0.001 & 0.004 & 0.326 & 0.744 & 0.021 \\
\hline PCS_w2 & $<--$ & Age_w1 & -0.067 & 0.035 & -1.894 & 0.058 & -0.120 \\
\hline PLIW_w1 & $<--$ & Age_w1 & -0.006 & 0.003 & -1.932 & 0.053 & -0.133 \\
\hline BoundaryOrg1_w1 & $<--$ & BoundaryOrg & 1 & & & & 0.914 \\
\hline BoundaryOrg2_w1 & $<--$ & BoundaryOrg & 0.946 & 0.034 & 27.873 & 0.000 & 0.925 \\
\hline BoundaryOrg3_w1 & $<--$ & BoundaryOrg & 0.932 & 0.033 & 28.217 & 0.000 & 0.929 \\
\hline BoundaryOrg4_w1 & $<--$ & BoundaryOrg & 0.898 & 0.037 & 24.314 & 0.000 & 0.879 \\
\hline BoundaryPref1_w1 & $<--$ & BoundaryPref & 1 & & & & 0.910 \\
\hline BoundaryPref2_w1 & $<--$ & BoundaryPref & 0.934 & 0.038 & 24.297 & 0.000 & 0.899 \\
\hline BoundaryPref3_w1 & $<-$ & BoundaryPref & 0.857 & 0.039 & 21.761 & 0.000 & 0.855 \\
\hline BoundaryPref4_w1 & $<--$ & BoundaryPref & 0.821 & 0.039 & 20.913 & 0.000 & 0.839 \\
\hline BoundaryOrg & $<--$ & Congruence & -0.500 & & & & -0.607 \\
\hline BoundaryPref & $<--$ & Congruence & 0.500 & & & & 0.693 \\
\hline Enhance_WtN_Val_w1 & $<--$ & Congruence & -0.079 & 0.023 & -3.455 & 0.000 & -0.212 \\
\hline PCS_w2 & $<--$ & Congruence & 0.395 & 0.194 & 2.037 & 0.042 & 0.121 \\
\hline PLIW_w1 & $<--$ & Congruence & 0.013 & 0.016 & 0.793 & 0.428 & 0.050 \\
\hline Enhance_WtN_Val_w1 & $<--$ & Education_w1 & 0.001 & 0.035 & 0.021 & 0.984 & 0.001 \\
\hline PCS_w2 & $<--$ & Education_w1 & 0.589 & 0.293 & 2.011 & 0.044 & 0.117 \\
\hline PLIW_w1 & $<--$ & Education_w1 & 0.020 & 0.026 & 0.792 & 0.429 & 0.051 \\
\hline Enhance_WtN_Val1_w1 & $<--$ & Enhance_WtN_Val_w1 & 1 & & & & 0.820 \\
\hline Enhance_WtN_Val2_w1 & $<--$ & Enhance_WtN_Val_w1 & 0.966 & 0.055 & 17.645 & 0.000 & 0.859 \\
\hline Enhance_WtN_Val3_w1 & $<--$ & Enhance_WtN_Val_w1 & 1.025 & 0.056 & 18.445 & 0.000 & 0.914 \\
\hline PCS_w2 & $<--$ & Enhance_WtN_Val_w1 & 0.569 & 0.522 & 1.090 & 0.276 & 0.066 \\
\hline Enhance_WtN_Val_w1 & $<--$ & Income_w2 & 0.004 & 0.027 & 0.135 & 0.893 & 0.009 \\
\hline PCS_w2 & $<--$ & Income_w2 & -0.152 & 0.220 & -0.693 & 0.488 & -0.043 \\
\hline PLIW_w1 & $<--$ & Income_w2 & -0.006 & 0.019 & -0.291 & 0.771 & -0.020 \\
\hline Enhance_WtN_Val_w1 & $<--$ & KidsAdult_w1 & 0.217 & 0.112 & 1.936 & 0.053 & 0.123 \\
\hline PCS_w2 & $<--$ & KidsAdult_w1 & -0.948 & 0.934 & -1.015 & 0.310 & -0.062 \\
\hline PLIW_w1 & $<--$ & KidsAdult_w1 & 0.050 & 0.081 & 0.619 & 0.536 & 0.041 \\
\hline BoundaryOrg & $<--$ & Level & 1 & & & & 0.718 \\
\hline BoundaryPref & $<--$ & Level & 1 & & & & 0.819 \\
\hline Enhance_WtN_Val_w1 & $<--$ & Level & -0.114 & 0.039 & -2.925 & 0.003 & -0.180 \\
\hline PCS_w2 & $<--$ & Level & -0.510 & 0.327 & -1.561 & 0.118 & -0.093 \\
\hline PLIW_w1 & $<--$ & Level & -0.011 & 0.028 & -0.377 & 0.706 & -0.024 \\
\hline PCS_w2 & $<--$ & PLIW_w1 & -0.377 & 0.740 & -0.510 & 0.610 & -0.030 \\
\hline PLIW1_w1 & $<--$ & PLIW_w1 & 1 & & & & 0.648 \\
\hline PLIW2_w1 & $<--$ & PLIW_w1 & 1.139 & 0.095 & 12.023 & 0.000 & 0.843 \\
\hline PLIW3_w1 & $<--$ & PLIW_w1 & 0.954 & 0.110 & 8.665 & 0.000 & 0.557 \\
\hline PLIW4_w1 & $<--$ & PLIW_w1 & 1.156 & 0.099 & 11.672 & 0.000 & 0.805 \\
\hline PLIW5_w1 & $<--$ & PLIW_w1 & 0.918 & 0.113 & 8.119 & 0.000 & 0.517 \\
\hline PLIW6_w1 & $<--$ & PLIW_w1 & 1.028 & 0.091 & 11.317 & 0.000 & 0.772 \\
\hline
\end{tabular}




\section{Model 11. WIPL, WtN Val, PCS}

Work-to-nonwork conflict, work-to-nonwork value-based enhancement, and physical health

\section{Unstandardized Output:}

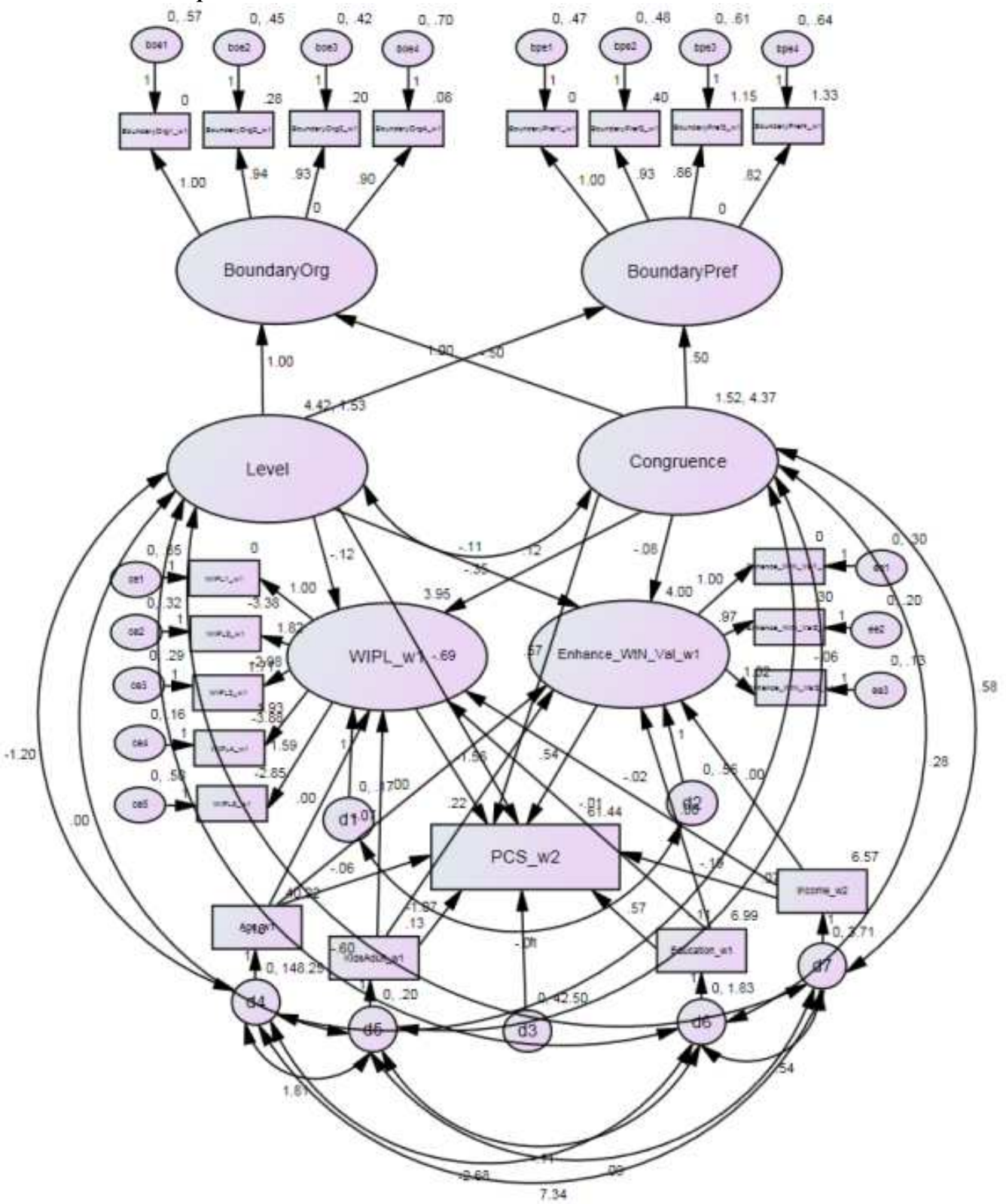

Fit Statistics:

- $\chi^{2}(158)=261.941 p<.001$

- CFI: .975

- RMSEA: .046 
Model 11 Output

\begin{tabular}{|c|c|c|c|c|c|c|c|}
\hline & & & Est. & S.E. & C.R. & $\mathrm{p}$ & $\begin{array}{l}\text { Std. } \\
\text { Est. }\end{array}$ \\
\hline Enhance_WtN_Val_w1 & $<--$ & Age_w1 & 0.001 & 0.004 & 0.318 & 0.751 & 0.021 \\
\hline PCS_w2 & $<--$ & Age_w1 & -0.063 & 0.035 & -1.810 & 0.070 & -0.113 \\
\hline WIPL_w1 & $<--$ & Age_w1 & 0.001 & 0.002 & 0.296 & 0.767 & 0.017 \\
\hline BoundaryOrg1_w1 & $<--$ & BoundaryOrg & 1 & & & & 0.916 \\
\hline BoundaryOrg2_w1 & $<--$ & BoundaryOrg & 0.945 & 0.034 & 28.074 & 0.000 & 0.924 \\
\hline BoundaryOrg3_w1 & $<--$ & BoundaryOrg & 0.930 & 0.033 & 28.327 & 0.000 & 0.928 \\
\hline BoundaryOrg4_w1 & $<--$ & BoundaryOrg & 0.896 & 0.037 & 24.415 & 0.000 & 0.879 \\
\hline BoundaryPref1_w1 & $<--$ & BoundaryPref & 1 & & & & 0.910 \\
\hline BoundaryPref2_w1 & $<--$ & BoundaryPref & 0.933 & 0.038 & 24.274 & 0.000 & 0.898 \\
\hline BoundaryPref3_w1 & $<--$ & BoundaryPref & 0.857 & 0.039 & 21.791 & 0.000 & 0.855 \\
\hline BoundaryPref4_w1 & $<--$ & BoundaryPref & 0.821 & 0.039 & 20.948 & 0.000 & 0.839 \\
\hline BoundaryOrg & $<--$ & Congruence & -0.500 & & & & -0.607 \\
\hline BoundaryPref & $<--$ & Congruence & 0.500 & & & & 0.693 \\
\hline Enhance_WtN_Val_w1 & $<--$ & Congruence & -0.079 & 0.023 & -3.455 & 0.000 & -0.212 \\
\hline PCS_w2 & $<--$ & Congruence & 0.568 & 0.225 & 2.521 & 0.012 & 0.175 \\
\hline WIPL_w1 & $<--$ & Congruence & 0.116 & 0.017 & 6.922 & 0.000 & 0.481 \\
\hline Enhance_WtN_Val_w1 & $<--$ & Education_w1 & 0 & 0.035 & 0.013 & 0.99 & 0.001 \\
\hline PCS_w2 & $<--$ & Education_w1 & 0.568 & 0.292 & 1.945 & 0.052 & 0.113 \\
\hline WIPL_w1 & $<--$ & Education_w1 & -0.010 & 0.020 & -0.487 & 0.626 & -0.026 \\
\hline Enhance_WtN_Val1_w1 & $<--$ & Enhance_WtN_Val_w1 & 1 & & & & 0.821 \\
\hline Enhance_WtN_Val2_w1 & $<--$ & Enhance_WtN_Val_w1 & 0.965 & 0.055 & 17.651 & 0.000 & 0.858 \\
\hline Enhance_WtN_Val3_w1 & $<--$ & Enhance_WtN_Val_w1 & 1.025 & 0.056 & 18.456 & 0.000 & 0.913 \\
\hline PCS_w2 & $<--$ & Enhance_WtN_Val_w1 & 0.544 & 0.520 & 1.047 & 0.295 & 0.063 \\
\hline Enhance_WtN_Val_w1 & $<--$ & Income_w2 & 0.004 & 0.027 & 0.152 & 0.879 & 0.010 \\
\hline PCS_w2 & $<--$ & Income_w2 & -0.188 & 0.220 & -0.853 & 0.394 & -0.053 \\
\hline WIPL_w1 & $<-$ & Income_w2 & -0.024 & 0.015 & -1.592 & 0.111 & -0.091 \\
\hline Enhance_WtN_Val_w1 & $<--$ & KidsAdult_w1 & 0.217 & 0.112 & 1.929 & 0.054 & 0.122 \\
\hline PCS_w2 & $<--$ & KidsAdult_w1 & -1.066 & 0.933 & -1.142 & 0.253 & -0.069 \\
\hline WIPL_w1 & $<--$ & KidsAdult_w1 & -0.066 & 0.062 & -1.061 & 0.289 & -0.058 \\
\hline BoundaryOrg & $<--$ & Level & 1 & & & & 0.718 \\
\hline BoundaryPref & $<--$ & Level & 1 & & & & 0.820 \\
\hline Enhance_WtN_Val_w1 & $<--$ & Level & -0.114 & 0.039 & -2.929 & 0.003 & -0.181 \\
\hline PCS_w2 & $<--$ & Level & -0.691 & 0.346 & -1.997 & 0.046 & -0.126 \\
\hline WIPL_w1 & $<--$ & Level & -0.117 & 0.024 & -4.793 & 0.000 & -0.288 \\
\hline PCS_w2 & $<--$ & WIPL_w1 & -1.564 & 0.971 & -1.611 & 0.107 & -0.116 \\
\hline WIPL1_w1 & $<--$ & WIPL_w1 & 1 & & & & 0.528 \\
\hline WIPL2_w1 & $<--$ & WIPL_w1 & 1.823 & 0.185 & 9.877 & 0.000 & 0.852 \\
\hline WIPL3_w1 & $<--$ & WIPL_w1 & 1.707 & 0.173 & 9.847 & 0.000 & 0.846 \\
\hline WIPL4_w1 & $<--$ & WIPL_w1 & 1.925 & 0.189 & 10.207 & 0.000 & 0.926 \\
\hline WIPL5_w1 & $<--$ & WIPL_w1 & 1.585 & 0.171 & 9.265 & 0.000 & 0.748 \\
\hline
\end{tabular}




\section{Model 12. WIPL, WtN Val, MCS}

Work-to-nonwork conflict, work-to-nonwork value-based enhancement, and mental health

\section{Unstandardized Output:}

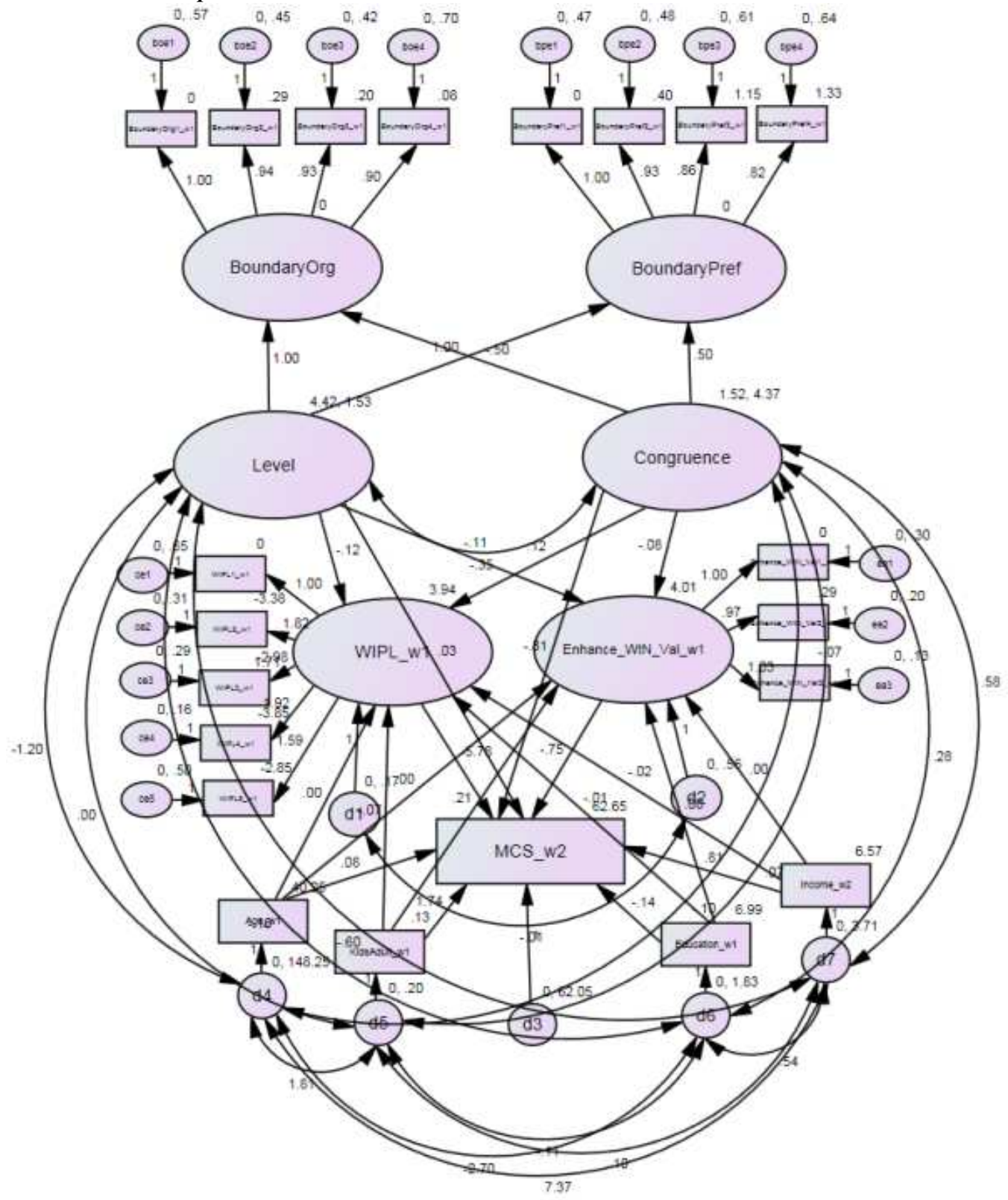

Fit Statistics:

- $\chi^{2}(158)=250.115 p<.001$

- CFI: .978

- RMSEA: .043 


\section{Model 12 Output}

\begin{tabular}{|c|c|c|c|c|c|c|c|}
\hline & & & Est. & S.E. & C.R. & $\mathrm{P}$ & $\begin{array}{l}\text { Std. } \\
\text { Est. }\end{array}$ \\
\hline Enhance_WtN_Val_w1 & $<--$ & Age_w1 & 0.001 & 0.004 & 0.322 & 0.748 & 0.021 \\
\hline MCS_w2 & $<--$ & Age_w1 & 0.082 & 0.042 & 1.940 & 0.052 & 0.110 \\
\hline WIPL_w1 & $<--$ & Age_w1 & 0.001 & 0.002 & 0.312 & 0.755 & 0.018 \\
\hline BoundaryOrg1_w1 & $<--$ & BoundaryOrg & 1 & & & & 0.916 \\
\hline BoundaryOrg2_w1 & $<--$ & BoundaryOrg & 0.944 & 0.034 & 28.068 & 0.000 & 0.924 \\
\hline BoundaryOrg3_w1 & $<--$ & BoundaryOrg & 0.929 & 0.033 & 28.339 & 0.000 & 0.928 \\
\hline BoundaryOrg4_w1 & $<--$ & BoundaryOrg & 0.896 & 0.037 & 24.439 & 0.000 & 0.879 \\
\hline BoundaryPref1_w1 & $<--$ & BoundaryPref & 1 & & & & 0.911 \\
\hline BoundaryPref2_w1 & $<--$ & BoundaryPref & 0.932 & 0.038 & 24.274 & 0.000 & 0.898 \\
\hline BoundaryPref3_w1 & $<--$ & BoundaryPref & 0.857 & 0.039 & 21.819 & 0.000 & 0.855 \\
\hline BoundaryPref4_w1 & $<--$ & BoundaryPref & 0.821 & 0.039 & 20.957 & 0.000 & 0.839 \\
\hline BoundaryOrg & $<--$ & Congruence & -0.500 & & & & -0.607 \\
\hline BoundaryPref & $<--$ & Congruence & 0.500 & & & & 0.693 \\
\hline Enhance_WtN_Val_w1 & $<--$ & Congruence & -0.079 & 0.023 & -3.45 & 0.000 & -0.212 \\
\hline MCS_w2 & $<--$ & Congruence & -0.812 & 0.274 & -2.965 & 0.003 & -0.187 \\
\hline WIPL_w1 & $<--$ & Congruence & 0.116 & 0.017 & 6.928 & 0.000 & 0.482 \\
\hline Enhance_WtN_Val_w1 & $<--$ & Education_w1 & 0.001 & 0.035 & 0.026 & 0.980 & 0.002 \\
\hline MCS_w2 & $<--$ & Education_w1 & -0.142 & 0.355 & -0.401 & 0.688 & -0.021 \\
\hline WIPL_w1 & $<--$ & Education_w1 & -0.009 & 0.020 & -0.477 & 0.633 & -0.025 \\
\hline Enhance_WtN_Val1_w1 & $<--$ & Enhance_WtN_Val_w1 & 1 & & & & 0.819 \\
\hline Enhance_WtN_Val2_w1 & $<--$ & Enhance_WtN_Val_w1 & 0.968 & 0.055 & 17.614 & 0.000 & 0.859 \\
\hline Enhance_WtN_Val3_w1 & $<--$ & Enhance_WtN_Val_w1 & 1.028 & 0.056 & 18.418 & 0.000 & 0.915 \\
\hline MCS_w2 & $<--$ & Enhance_WtN_Val_w1 & -0.752 & 0.633 & -1.189 & 0.235 & -0.065 \\
\hline Enhance_WtN_Val_w1 & $<--$ & Income_w2 & 0.003 & 0.027 & 0.106 & 0.916 & 0.007 \\
\hline MCS_w2 & $<--$ & Income_w2 & 0.814 & 0.268 & 3.043 & 0.002 & 0.173 \\
\hline WIPL_w1 & $<--$ & Income_w2 & -0.024 & 0.015 & -1.583 & 0.113 & -0.091 \\
\hline Enhance_WtN_Val_w1 & $<--$ & KidsAdult_w1 & 0.214 & 0.112 & 1.914 & 0.056 & 0.121 \\
\hline MCS_w2 & $<--$ & KidsAdult_w1 & 1.744 & 1.133 & 1.539 & 0.124 & 0.085 \\
\hline WIPL_w1 & $<--$ & KidsAdult_w1 & -0.066 & 0.063 & -1.061 & 0.289 & -0.058 \\
\hline BoundaryOrg & $<-$ & Level & 1 & & & & 0.718 \\
\hline BoundaryPref & $<--$ & Level & 1 & & & & 0.819 \\
\hline Enhance_WtN_Val_w1 & $<--$ & Level & -0.115 & 0.039 & -2.945 & 0.003 & -0.182 \\
\hline MCS_w2 & $<--$ & Level & 0.034 & 0.420 & 0.082 & 0.935 & 0.005 \\
\hline WIPL_w1 & $<--$ & Level & -0.117 & 0.024 & -4.788 & 0.000 & -0.287 \\
\hline MCS_w2 & $<--$ & WIPL_w1 & -5.783 & 1.283 & -4.507 & 0.000 & -0.320 \\
\hline WIPL1_w1 & $<--$ & WIPL_w1 & 1 & & & & 0.529 \\
\hline WIPL2_w1 & $<--$ & WIPL_w1 & 1.825 & 0.184 & 9.907 & 0.000 & 0.854 \\
\hline WIPL3_w1 & $<--$ & WIPL_w1 & 1.706 & 0.173 & 9.869 & 0.000 & 0.847 \\
\hline WIPL4_w1 & $<--$ & WIPL_w1 & 1.916 & 0.188 & 10.219 & 0.000 & 0.923 \\
\hline WIPL5_w1 & $<--$ & WIPL_w1 & 1.585 & 0.171 & 9.288 & 0.000 & 0.750 \\
\hline
\end{tabular}




\section{Model 13. WIPL, NtW Beh, MCS}

Work-to-nonwork conflict, nonwork-to-work behavior-based enhancement, and mental health

\section{Unstandardized Output:}

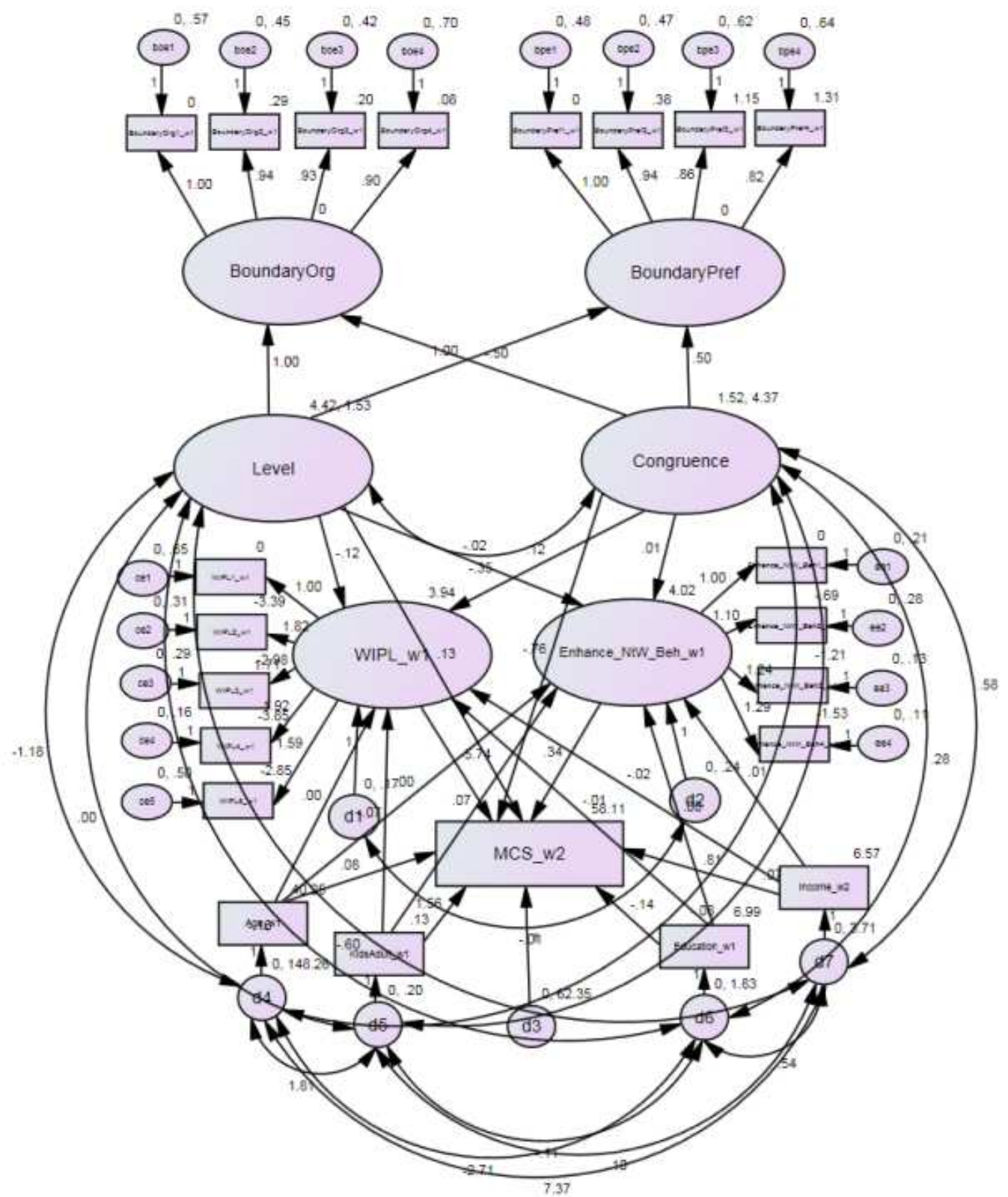

Fit Statistics:

- $\chi^{2}(178)=259.858 p<.001$

- CFI: .942

- RMSEA: .038 
Model 13 Output

\begin{tabular}{|c|c|c|c|c|c|c|c|}
\hline & & & Est. & S.E. & C.R. & $\mathrm{P}$ & $\begin{array}{l}\text { Std. } \\
\text { Est. }\end{array}$ \\
\hline Enhance_NtW_Beh_w1 & $<-$ & Age_w1 & 0.005 & 0.003 & 1.612 & 0.107 & 0.109 \\
\hline MCS_w2 & $<--$ & Age_w1 & 0.080 & 0.043 & 1.866 & 0.062 & 0.107 \\
\hline WIPL_w1 & $<--$ & Age_w1 & 0.001 & 0.002 & 0.338 & 0.735 & 0.019 \\
\hline BoundaryOrg1_w1 & $<-$ & BoundaryOrg & 1 & & & & 0.916 \\
\hline BoundaryOrg2_w1 & $<-$ & BoundaryOrg & 0.944 & 0.034 & 28.077 & 0.000 & 0.924 \\
\hline BoundaryOrg3_w1 & $<-$ & BoundaryOrg & 0.929 & 0.033 & 28.344 & 0.000 & 0.927 \\
\hline BoundaryOrg4_w1 & $<--$ & BoundaryOrg & 0.896 & 0.037 & 24.463 & 0.000 & 0.879 \\
\hline BoundaryPref1_w1 & $<-$ & BoundaryPref & 1 & & & & 0.909 \\
\hline BoundaryPref2_w1 & $<-$ & BoundaryPref & 0.936 & 0.039 & 24.265 & 0.000 & 0.899 \\
\hline BoundaryPref3_w1 & $<-$ & BoundaryPref & 0.857 & 0.04 & 21.677 & 0.000 & 0.854 \\
\hline BoundaryPref4_w1 & $<--$ & BoundaryPref & 0.824 & 0.039 & 20.952 & 0.000 & 0.841 \\
\hline BoundaryOrg & $<-$ & Congruence & -0.500 & & & & -0.606 \\
\hline BoundaryPref & $<-$ & Congruence & 0.500 & & & & 0.694 \\
\hline Enhance_NtW_Beh_w1 & $<--$ & Congruence & 0.007 & 0.015 & 0.440 & 0.660 & 0.027 \\
\hline MCS_w2 & $<-$ & Congruence & -0.760 & 0.270 & -2.813 & 0.005 & -0.175 \\
\hline WIPL_w1 & $<--$ & Congruence & 0.116 & 0.017 & 6.927 & 0.000 & 0.482 \\
\hline Enhance_NtW_Beh_w1 & $<--$ & Education_w1 & 0 & 0.023 & -0.011 & 0.991 & -0.001 \\
\hline MCS_w2 & $<--$ & Education_w1 & -0.143 & 0.355 & -0.402 & 0.688 & -0.021 \\
\hline WIPL_w1 & $<--$ & Education_w1 & -0.010 & 0.020 & -0.483 & 0.629 & -0.026 \\
\hline Enhance_NtW_Beh1_w1 & $<--$ & Enhance_NtW_Beh_w1 & 1 & & & & 0.738 \\
\hline Enhance_NtW_Beh2_w1 & $<--$ & Enhance_NtW_Beh_w1 & 1.099 & 0.088 & 12.426 & 0.000 & 0.722 \\
\hline Enhance_NtW_Beh3_w1 & $<-$ & Enhance_NtW_Beh_w1 & 1.242 & 0.084 & 14.875 & 0.000 & 0.863 \\
\hline Enhance_NtW_Beh4_w1 & $<--$ & Enhance_NtW_Beh_w1 & 1.293 & 0.085 & 15.182 & 0.000 & 0.889 \\
\hline MCS_w2 & $<--$ & Enhance_NtW_Beh_w1 & 0.336 & 0.963 & 0.349 & 0.727 & 0.019 \\
\hline Enhance_NtW_Beh_w1 & $<--$ & Income_w2 & 0.011 & 0.018 & 0.652 & 0.515 & 0.044 \\
\hline MCS_w2 & $<--$ & Income_w2 & 0.809 & 0.268 & 3.016 & 0.003 & 0.172 \\
\hline WIPL_w1 & $<--$ & Income_w2 & -0.024 & 0.015 & -1.586 & 0.113 & -0.091 \\
\hline Enhance_NtW_Beh_w1 & $<--$ & KidsAdult_w1 & 0.069 & 0.074 & 0.926 & 0.355 & 0.06 \\
\hline MCS_w2 & $<--$ & KidsAdult_w1 & 1.561 & 1.128 & 1.384 & 0.166 & 0.076 \\
\hline WIPL_w1 & $<--$ & KidsAdult_w1 & -0.070 & 0.063 & -1.116 & 0.264 & -0.061 \\
\hline BoundaryOrg & $<--$ & Level & 1 & & & & 0.717 \\
\hline BoundaryPref & $<--$ & Level & 1 & & & & 0.820 \\
\hline Enhance_NtW_Beh_w1 & $<--$ & Level & -0.023 & 0.026 & -0.916 & 0.360 & -0.058 \\
\hline MCS_w2 & $<--$ & Level & 0.134 & 0.415 & 0.322 & 0.748 & 0.018 \\
\hline WIPL_w1 & $<-$ & Level & -0.117 & 0.024 & -4.78 & 0.000 & -0.287 \\
\hline MCS_w2 & $<--$ & WIPL_w1 & -5.737 & 1.284 & -4.467 & 0.000 & -0.318 \\
\hline WIPL1_w1 & $<--$ & WIPL_w1 & 1 & & & & 0.529 \\
\hline WIPL2_w1 & $<--$ & WIPL_w1 & 1.825 & 0.184 & 9.905 & 0.000 & 0.854 \\
\hline WIPL3_w1 & $<--$ & WIPL_w1 & 1.705 & 0.173 & 9.866 & 0.000 & 0.847 \\
\hline WIPL4_w1 & $<--$ & WIPL_w1 & 1.917 & 0.188 & 10.218 & 0.000 & 0.924 \\
\hline WIPL5_w1 & $<--$ & WIPL_w1 & 1.586 & 0.171 & 9.286 & 0.000 & 0.750 \\
\hline
\end{tabular}




\section{Model 14. WIPL, NtW Beh, PCS}

Work-to-nonwork conflict, nonwork-to-work behavior-based enhancement, and physical health

\section{Unstandardized Output:}

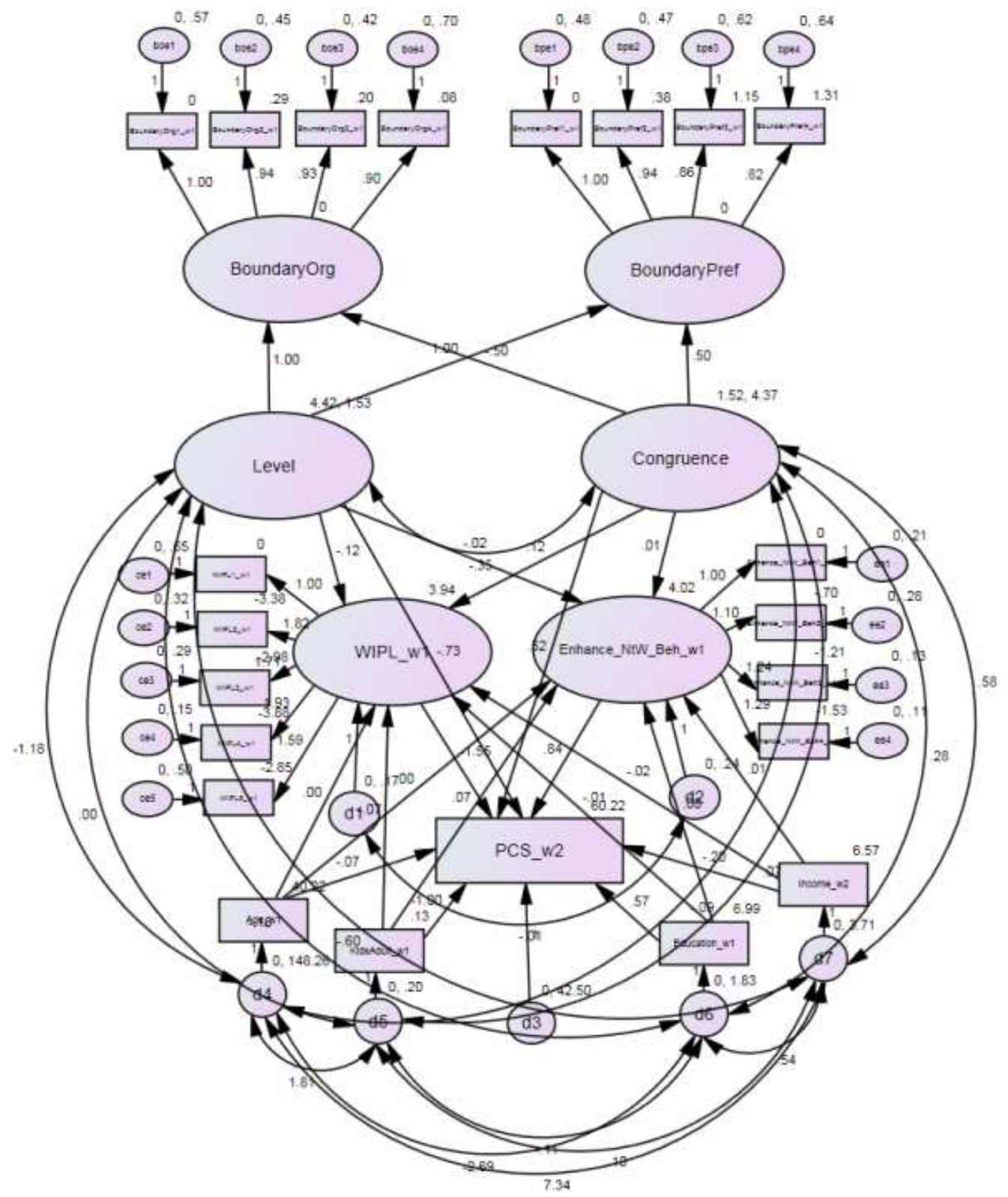

Fit Statistics:

- $\chi^{2}(178)=266.002 p<.001$

- CFI: 940

- RMSEA: .040 
Model 14 Output

\begin{tabular}{|c|c|c|c|c|c|c|c|}
\hline & & & Est. & S.E. & C.R. & $\mathrm{P}$ & $\begin{array}{l}\text { Std. } \\
\text { Est. }\end{array}$ \\
\hline Enhance_NtW_Beh_w1 & $<--$ & Age_w1 & 0.004 & 0.003 & 1.598 & 0.110 & 0.108 \\
\hline PCS_w2 & $<--$ & Age_w1 & -0.066 & 0.035 & -1.886 & 0.059 & -0.118 \\
\hline WIPL_w1 & $<--$ & Age_w1 & 0.001 & 0.002 & 0.325 & 0.745 & 0.018 \\
\hline BoundaryOrg1_w1 & $<--$ & BoundaryOrg & 1 & & & & 0.916 \\
\hline BoundaryOrg2_w1 & $<--$ & BoundaryOrg & 0.945 & 0.034 & 28.082 & 0.000 & 0.924 \\
\hline BoundaryOrg3_w1 & $<--$ & BoundaryOrg & 0.929 & 0.033 & 28.331 & 0.000 & 0.927 \\
\hline BoundaryOrg4_w1 & $<--$ & BoundaryOrg & 0.896 & 0.037 & 24.436 & 0.000 & 0.879 \\
\hline BoundaryPref1_w1 & $<--$ & BoundaryPref & 1 & & & & 0.909 \\
\hline BoundaryPref2_w1 & $<--$ & BoundaryPref & 0.936 & 0.039 & 24.260 & 0.000 & 0.900 \\
\hline BoundaryPref3_w1 & $<--$ & BoundaryPref & 0.858 & 0.040 & 21.656 & 0.000 & 0.854 \\
\hline BoundaryPref4_w1 & $<--$ & BoundaryPref & 0.824 & 0.039 & 20.941 & 0.000 & 0.841 \\
\hline BoundaryOrg & $<--$ & Congruence & -0.500 & & & & -0.606 \\
\hline BoundaryPref & $<--$ & Congruence & 0.500 & & & & 0.694 \\
\hline Enhance_NtW_Beh_w1 & $<--$ & Congruence & 0.006 & 0.015 & 0.429 & 0.668 & 0.027 \\
\hline PCS_w2 & $<--$ & Congruence & 0.517 & 0.222 & 2.333 & 0.020 & 0.159 \\
\hline WIPL_w1 & $<--$ & Congruence & 0.116 & 0.017 & 6.920 & 0.000 & 0.481 \\
\hline Enhance_NtW_Beh_w1 & $<--$ & Education_w1 & 0 & 0.023 & -0.019 & 0.985 & -0.001 \\
\hline PCS_w2 & $<--$ & Education_w1 & 0.569 & 0.292 & 1.950 & 0.051 & 0.113 \\
\hline WIPL_w1 & $<--$ & Education_w1 & -0.010 & 0.020 & -0.491 & 0.623 & -0.026 \\
\hline Enhance_NtW_Beh1_w1 & $<--$ & Enhance_NtW_Beh_w1 & 1 & & & & 0.738 \\
\hline Enhance_NtW_Beh2_w1 & $<--$ & Enhance_NtW_Beh_w1 & 1.101 & 0.089 & 12.442 & 0.000 & 0.723 \\
\hline Enhance_NtW_Beh3_w1 & $<--$ & Enhance_NtW_Beh_w1 & 1.243 & 0.084 & 14.873 & 0.000 & 0.863 \\
\hline Enhance_NtW_Beh4_w1 & $<--$ & Enhance_NtW_Beh_w1 & 1.292 & 0.085 & 15.168 & 0.000 & 0.889 \\
\hline PCS_w2 & $<--$ & Enhance_NtW_Beh_w1 & 0.836 & 0.792 & 1.055 & 0.291 & 0.062 \\
\hline Enhance_NtW_Beh_w1 & $<--$ & Income_w2 & 0.012 & 0.018 & 0.691 & 0.490 & 0.047 \\
\hline PCS_w2 & $<--$ & Income_w2 & -0.197 & 0.221 & -0.895 & 0.371 & -0.056 \\
\hline WIPL_w1 & $<--$ & Income_w2 & -0.024 & 0.015 & -1.597 & 0.110 & -0.091 \\
\hline Enhance_NtW_Beh_w1 & $<--$ & KidsAdult_w1 & 0.070 & 0.074 & 0.947 & 0.344 & 0.062 \\
\hline PCS_w2 & $<--$ & KidsAdult_w1 & -1.001 & 0.927 & -1.080 & 0.280 & -0.065 \\
\hline WIPL_w1 & $<--$ & KidsAdult_w1 & -0.070 & 0.062 & -1.115 & 0.265 & -0.061 \\
\hline BoundaryOrg & $<--$ & Level & 1 & & & & 0.717 \\
\hline BoundaryPref & $<--$ & Level & 1 & & & & 0.820 \\
\hline Enhance_NtW_Beh_w1 & $<--$ & Level & -0.023 & 0.026 & -0.908 & 0.364 & -0.057 \\
\hline PCS_w2 & $<--$ & Level & -0.733 & 0.341 & -2.150 & 0.032 & -0.133 \\
\hline WIPL_w1 & $<--$ & Level & -0.117 & 0.024 & -4.786 & 0.000 & -0.288 \\
\hline PCS_w2 & $<--$ & WIPL_w1 & -1.552 & 0.972 & -1.596 & 0.110 & -0.115 \\
\hline WIPL1_w1 & $<--$ & WIPL_w1 & 1 & & & & 0.528 \\
\hline WIPL2_w1 & $<--$ & WIPL_w1 & 1.824 & 0.185 & 9.873 & 0.000 & 0.852 \\
\hline WIPL3_w1 & $<--$ & WIPL_w1 & 1.707 & 0.173 & 9.843 & 0.000 & 0.846 \\
\hline WIPL4_w1 & $<--$ & WIPL_w1 & 1.926 & 0.189 & 10.204 & 0.000 & 0.926 \\
\hline WIPL5_w1 & $<--$ & WIPL_w1 & 1.586 & 0.171 & 9.262 & 0.000 & 0.748 \\
\hline
\end{tabular}




\section{Model 15. PLIW, NtW Beh, PCS}

Nonwork-to-work conflict, nonwork-to-work behavior-based enhancement, and physical health

\section{Unstandardized Output:}

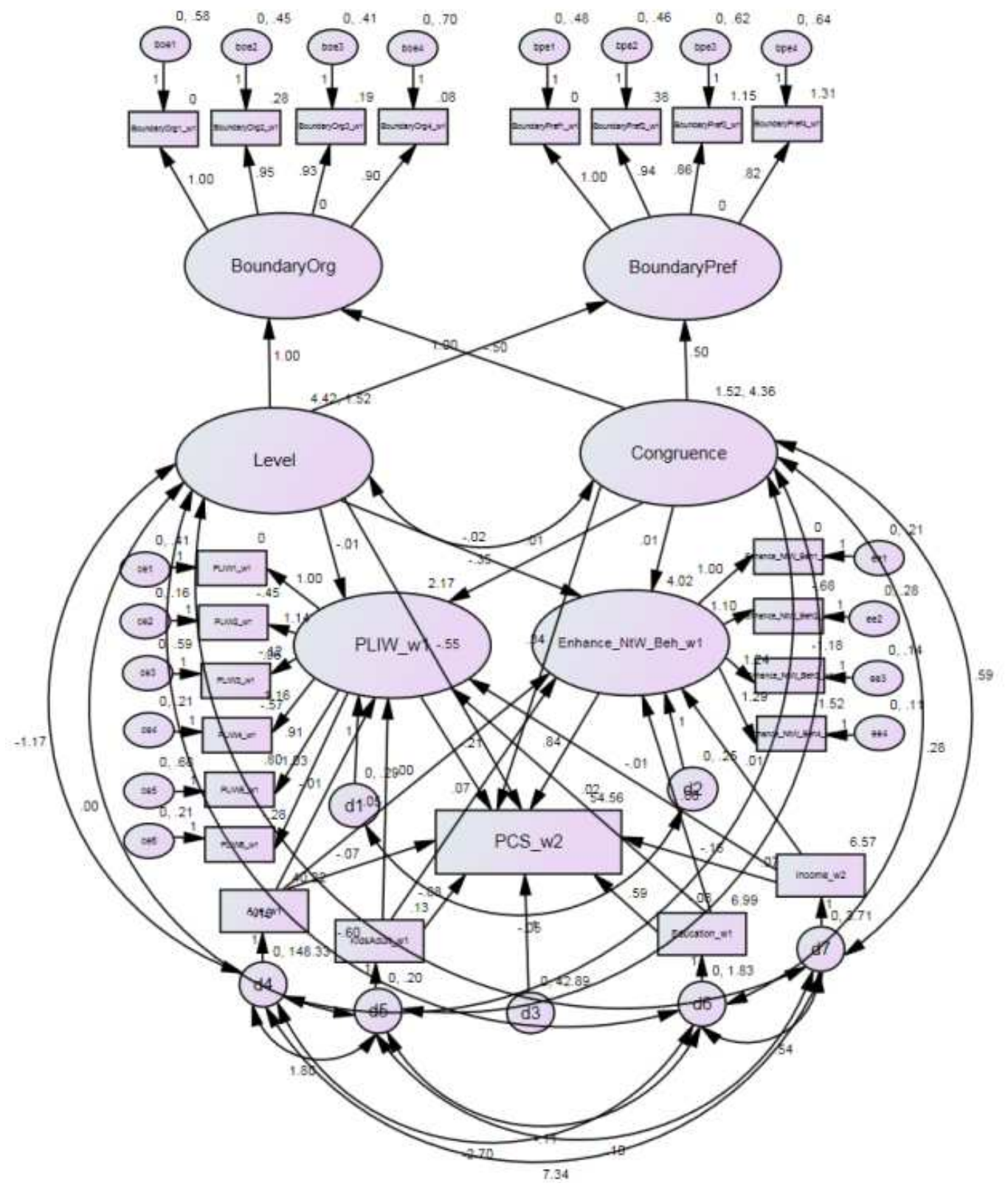

Fit Statistics:

- $\chi^{2}(199)=319.023, p<.001$

- CFI: .944

- RMSEA: .044 
Model 15 Output

\begin{tabular}{|c|c|c|c|c|c|c|c|}
\hline & & & Est. & S.E. & C.R. & $\mathrm{p}$ & $\begin{array}{l}\text { Std. } \\
\text { Est. }\end{array}$ \\
\hline Enhance_NtW_Beh_w1 & $<-$ & Age_w1 & 0.004 & 0.003 & 1.589 & 0.112 & 0.108 \\
\hline PCS_w2 & $<-$ & Age_w1 & -0.069 & 0.035 & -1.944 & 0.052 & -0.123 \\
\hline PLIW_w1 & $<-$ & Age_w1 & -0.006 & 0.003 & -1.949 & 0.051 & -0.135 \\
\hline BoundaryOrg1_w1 & $<-$ & BoundaryOrg & 1 & & & & 0.914 \\
\hline BoundaryOrg2_w1 & $<-$ & BoundaryOrg & 0.946 & 0.034 & 27.884 & 0.000 & 0.924 \\
\hline BoundaryOrg3_w1 & $<-$ & BoundaryOrg & 0.932 & 0.033 & 28.222 & 0.000 & 0.929 \\
\hline BoundaryOrg4_w1 & $<-$ & BoundaryOrg & 0.898 & 0.037 & 24.337 & 0.000 & 0.879 \\
\hline BoundaryPref1_w1 & $<-$ & BoundaryPref & 1 & & & & 0.909 \\
\hline BoundaryPref2_w1 & $<-$ & BoundaryPref & 0.938 & 0.039 & 24.281 & 0.000 & 0.901 \\
\hline BoundaryPref3_w1 & $<-$ & BoundaryPref & 0.858 & 0.040 & 21.623 & 0.000 & 0.854 \\
\hline BoundaryPref4_w1 & $<-$ & BoundaryPref & 0.824 & 0.039 & 20.903 & 0.000 & 0.840 \\
\hline BoundaryOrg & $<-$ & Congruence & -0.500 & & & & -0.607 \\
\hline BoundaryPref & $<-$ & Congruence & 0.500 & & & & 0.694 \\
\hline Enhance_NtW_Beh_w1 & $<-$ & Congruence & 0.006 & 0.015 & 0.374 & 0.708 & 0.023 \\
\hline PCS_w2 & $<-$ & Congruence & 0.342 & 0.189 & 1.811 & 0.070 & 0.105 \\
\hline PLIW_w1 & $<-$ & Congruence & 0.013 & 0.016 & 0.790 & 0.430 & 0.050 \\
\hline Enhance_NtW_Beh_w1 & $<-$ & Education_w1 & -0.001 & 0.023 & -0.031 & 0.975 & -0.002 \\
\hline PCS_w2 & $<-$ & Education_w1 & 0.588 & 0.293 & 2.007 & 0.045 & 0.117 \\
\hline PLIW_w1 & $<-$ & Education_w1 & 0.020 & 0.026 & 0.796 & 0.426 & 0.051 \\
\hline Enhance_NtW_Beh1_w1 & $<-$ & Enhance_NtW_Beh_w1 & 1 & & & & 0.740 \\
\hline Enhance_NtW_Beh2_w1 & $<-$ & Enhance_NtW_Beh_w1 & 1.097 & 0.088 & 12.463 & 0.000 & 0.722 \\
\hline Enhance_NtW_Beh3_w1 & $<-$ & Enhance_NtW_Beh_w1 & 1.235 & 0.083 & 14.899 & 0.000 & 0.861 \\
\hline Enhance_NtW_Beh4_w1 & $<-$ & Enhance_NtW_Beh_w1 & 1.292 & 0.085 & 15.261 & 0.000 & 0.890 \\
\hline PCS_w2 & $<-$ & Enhance_NtW_Beh_w1 & 0.838 & 0.809 & 1.036 & 0.300 & 0.062 \\
\hline Enhance_NtW_Beh_w1 & $<-$ & Income_w2 & 0.012 & 0.018 & 0.700 & 0.484 & 0.047 \\
\hline PCS_w2 & $<-$ & Income_w2 & -0.162 & 0.220 & -0.735 & 0.462 & -0.046 \\
\hline PLIW_w1 & $<-$ & Income_w2 & -0.005 & 0.019 & -0.285 & 0.776 & -0.019 \\
\hline Enhance_NtW_Beh_w1 & $<-$ & KidsAdult_w1 & 0.069 & 0.074 & 0.929 & 0.353 & 0.061 \\
\hline PCS_w2 & $<-$ & KidsAdult_w1 & -0.883 & 0.929 & -0.950 & 0.342 & -0.057 \\
\hline PLIW_w1 & $<-$ & KidsAdult_w1 & 0.053 & 0.081 & 0.656 & 0.512 & 0.043 \\
\hline BoundaryOrg & $<-$ & Level & 1 & & & & 0.717 \\
\hline BoundaryPref & $<-$ & Level & 1 & & & & 0.820 \\
\hline Enhance_NtW_Beh_w1 & $<-$ & Level & -0.023 & 0.026 & -0.910 & 0.363 & -0.057 \\
\hline PCS_w2 & $<-$ & Level & -0.555 & 0.322 & -1.724 & 0.085 & -0.101 \\
\hline PLIW_w1 & $<-$ & Level & -0.010 & 0.028 & -0.375 & 0.707 & -0.024 \\
\hline PCS_w2 & $<-$ & PLIW_w1 & -0.212 & 0.756 & -0.281 & 0.779 & -0.017 \\
\hline PLIW1_w1 & $<-$ & PLIW_w1 & 1 & & & & 0.648 \\
\hline PLIW2_w1 & $<-$ & PLIW_w1 & 1.139 & 0.095 & 12.014 & 0.000 & 0.842 \\
\hline PLIW3_w1 & $<-$ & PLIW_w1 & 0.956 & 0.110 & 8.673 & 0.000 & 0.558 \\
\hline PLIW4_w1 & $<-$ & PLIW_w1 & 1.159 & 0.099 & 11.684 & 0.000 & 0.806 \\
\hline PLIW5_w1 & $<-$ & PLIW_w1 & 0.913 & 0.113 & 8.069 & 0.000 & 0.514 \\
\hline PLIW6_w1 & $<-$ & PLIW_w1 & 1.029 & 0.091 & 11.317 & 0.000 & 0.773 \\
\hline
\end{tabular}




\section{Model 16. PLIW, NtW Beh, MCS}

Nonwork-to-work conflict, nonwork-to-work behavior-based enhancement, and mental health

\section{Unstandardized Output:}

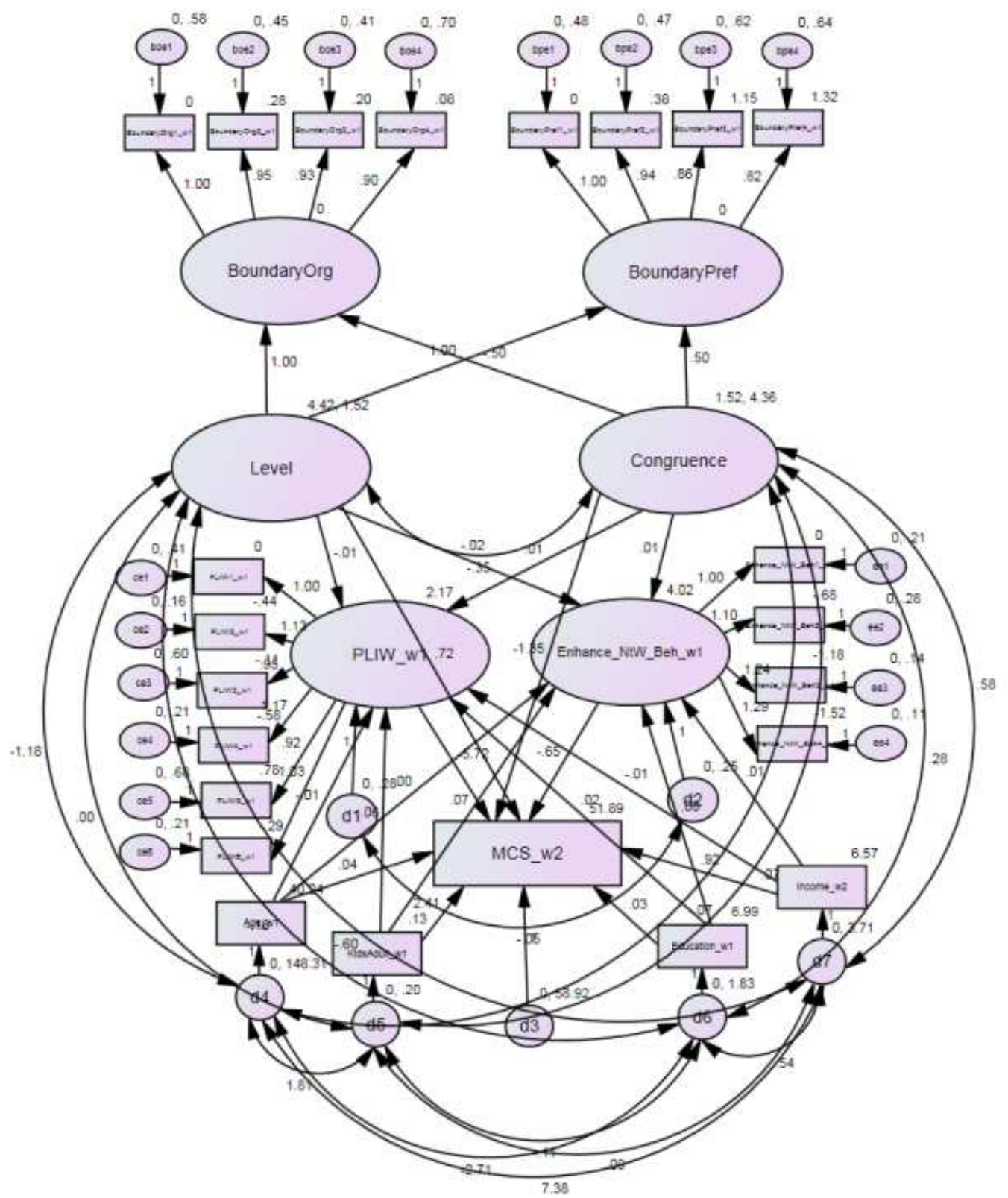

Fit Statistics:

- $\chi^{2}(199)=308.61, p<.001$

- CFI: 972

- RMSEA: .042 


\begin{tabular}{|c|c|c|c|c|c|c|c|}
\hline & & & Est. & S.E. & C.R. & $\mathrm{P}$ & Std. Est. \\
\hline Enhance_NtW_Beh_w1 & $<-$ & Age_w1 & 0.004 & 0.003 & 1.592 & 0.111 & 0.108 \\
\hline MCS_w2 & $<-$ & Age_w1 & 0.044 & 0.042 & 1.046 & 0.296 & 0.059 \\
\hline PLIW_w1 & $<-$ & Age_w1 & -0.006 & 0.003 & -1.971 & 0.049 & -0.136 \\
\hline BoundaryOrg1_w1 & $<--$ & BoundaryOrg & 1 & & & & 0.915 \\
\hline BoundaryOrg2_w1 & $<-$ & BoundaryOrg & 0.945 & 0.034 & 27.978 & $* * *$ & 0.924 \\
\hline BoundaryOrg3_w1 & $<-$ & BoundaryOrg & 0.931 & 0.033 & 28.272 & $* * *$ & 0.928 \\
\hline BoundaryOrg4_w1 & $<-$ & BoundaryOrg & 0.896 & 0.037 & 24.361 & $* * *$ & 0.879 \\
\hline BoundaryPref1_w1 & $<-$ & BoundaryPref & 1 & & & & 0.909 \\
\hline BoundaryPref2_w1 & $<-$ & BoundaryPref & 0.936 & 0.039 & 24.275 & $* * *$ & 0.9 \\
\hline BoundaryPref3_w1 & $<-$ & BoundaryPref & 0.857 & 0.04 & 21.662 & $* * *$ & 0.854 \\
\hline BoundaryPref4_w1 & $<--$ & BoundaryPref & 0.823 & 0.039 & 20.944 & $* * *$ & 0.84 \\
\hline BoundaryOrg & $<--$ & Congruence & -0.5 & & & & -0.607 \\
\hline BoundaryPref & $<-$ & Congruence & 0.5 & & & & 0.694 \\
\hline Enhance_NtW_Beh_w1 & $<-$ & Congruence & 0.006 & 0.015 & 0.372 & 0.71 & 0.023 \\
\hline MCS_w2 & $<--$ & Congruence & -1.346 & 0.226 & -5.969 & $* * *$ & -0.309 \\
\hline PLIW_w1 & $<--$ & Congruence & 0.013 & 0.016 & 0.796 & 0.426 & 0.05 \\
\hline Enhance_NtW_Beh_w1 & $<--$ & Education_w1 & -0.001 & 0.023 & -0.037 & 0.97 & -0.002 \\
\hline MCS_w2 & $<--$ & Education_w1 & 0.032 & 0.349 & 0.091 & 0.927 & 0.005 \\
\hline PLIW_w1 & $<-$ & Education_w1 & 0.021 & 0.025 & 0.82 & 0.412 & 0.052 \\
\hline Enhance_NtW_Beh1_w1 & $<--$ & Enhance_NtW_Beh_w1 & 1 & & & & 0.74 \\
\hline Enhance_NtW_Beh2_w1 & $<--$ & Enhance_NtW_Beh_w1 & 1.096 & 0.088 & 12.46 & $* * *$ & 0.721 \\
\hline Enhance_NtW_Beh3_w1 & $<-$ & Enhance_NtW_Beh_w1 & 1.235 & 0.083 & 14.901 & $* * *$ & 0.861 \\
\hline Enhance_NtW_Beh4_w1 & $<-$ & Enhance_NtW_Beh_w1 & 1.292 & 0.085 & 15.262 & $* * *$ & 0.89 \\
\hline MCS_w2 & $<-$ & Enhance_NtW_Beh_w1 & -0.647 & 0.962 & -0.672 & 0.501 & -0.036 \\
\hline Enhance_NtW_Beh_w1 & $<-$ & Income_w2 & 0.012 & 0.018 & 0.696 & 0.486 & 0.047 \\
\hline MCS_w2 & $<--$ & Income_w2 & 0.921 & 0.262 & 3.521 & $* * *$ & 0.195 \\
\hline PLIW_w1 & $<-$ & Income_w2 & -0.006 & 0.019 & -0.321 & 0.748 & -0.022 \\
\hline Enhance_NtW_Beh_w1 & $<-$ & KidsAdult_w1 & 0.065 & 0.074 & 0.879 & 0.379 & 0.057 \\
\hline MCS_w2 & $<-$ & KidsAdult_w1 & 2.412 & 1.105 & 2.182 & 0.029 & 0.117 \\
\hline PLIW_w1 & $<-$ & KidsAdult_w1 & 0.057 & 0.081 & 0.706 & 0.48 & 0.047 \\
\hline BoundaryOrg & $<-$ & Level & 1 & & & & 0.717 \\
\hline BoundaryPref & $<-$ & Level & 1 & & & & 0.82 \\
\hline Enhance_NtW_Beh_w1 & $<-$ & Level & -0.024 & 0.026 & -0.918 & 0.358 & -0.058 \\
\hline MCS_w2 & $<-$ & Level & 0.721 & 0.385 & 1.873 & 0.061 & 0.098 \\
\hline PLIW_w1 & $<-$ & Level & -0.011 & 0.028 & -0.383 & 0.702 & -0.024 \\
\hline MCS_w2 & $<-$ & PLIW_w1 & -5.718 & 0.983 & -5.816 & $* * *$ & -0.34 \\
\hline PLIW1_w1 & $<-$ & PLIW_w1 & 1 & & & & 0.647 \\
\hline PLIW2_w1 & $<-$ & PLIW_w1 & 1.134 & 0.095 & 11.999 & $* * *$ & 0.838 \\
\hline PLIW3_w1 & $<-$ & PLIW_w1 & 0.953 & 0.11 & 8.649 & $* * *$ & 0.555 \\
\hline PLIW4_w1 & $<-$ & PLIW_w1 & 1.166 & 0.099 & 11.743 & $* * *$ & 0.811 \\
\hline PLIW5_w1 & & PLIW_w1 & 0.92 & 0.113 & 8.125 & $* * *$ & 0.517 \\
\hline PLIW6_w1 & $<-$ & PLIW_w1 & 1.03 & 0.091 & 11.332 & $* * *$ & 0.773 \\
\hline
\end{tabular}




\section{Model 17. PLIW, NtW Aff, PCS}

Nonwork-to-work conflict, nonwork-to-work affective enhancement, and physical health

\section{Unstandardized Output:}

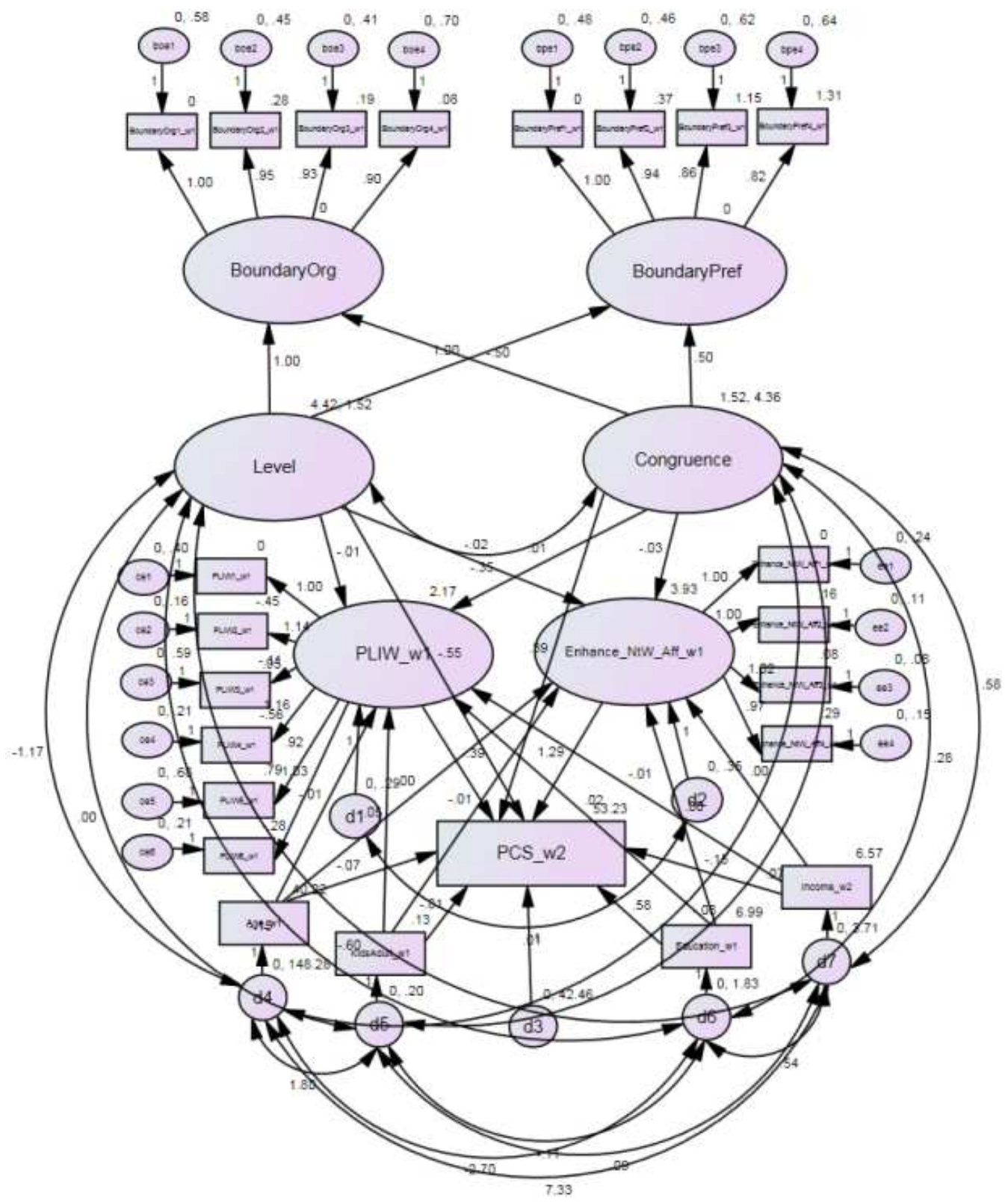

Fit Statistics:

- $\chi^{2}(199)=308.97, p<.001$

- CFI: .974

- RMSEA: .042 
Model 17 Output

\begin{tabular}{|c|c|c|c|c|c|c|c|}
\hline & & & Est. & S.E. & C.R. & $\mathrm{P}$ & $\begin{array}{l}\text { Std. } \\
\text { Est. }\end{array}$ \\
\hline Enhance_NtW_Aff_w1 & $<-$ & Age_w1 & 0.002 & 0.003 & 0.473 & 0.636 & 0.032 \\
\hline PCS_w2 & $<-$ & Age_w1 & -0.068 & 0.035 & -1.94 & 0.052 & -0.122 \\
\hline PLIW_w1 & $<-$ & Age_w1 & -0.006 & 0.003 & -1.948 & 0.051 & -0.134 \\
\hline BoundaryOrg1_w1 & $<-$ & BoundaryOrg & 1 & & & & 0.914 \\
\hline BoundaryOrg2_w1 & $<-$ & BoundaryOrg & 0.947 & 0.034 & 27.881 & $* * *$ & 0.925 \\
\hline BoundaryOrg3_w1 & $<--$ & BoundaryOrg & 0.933 & 0.033 & 28.217 & $* * *$ & 0.929 \\
\hline BoundaryOrg4_w1 & $<-$ & BoundaryOrg & 0.898 & 0.037 & 24.299 & $* * *$ & 0.879 \\
\hline BoundaryPref1_w1 & $<-$ & BoundaryPref & 1 & & & & 0.909 \\
\hline BoundaryPref2_w1 & $<-$ & BoundaryPref & 0.938 & 0.039 & 24.298 & $* * *$ & 0.901 \\
\hline BoundaryPref3_w1 & $<--$ & BoundaryPref & 0.857 & 0.04 & 21.605 & $* * *$ & 0.854 \\
\hline BoundaryPref4_w1 & $<--$ & BoundaryPref & 0.824 & 0.039 & 20.914 & $* * *$ & 0.84 \\
\hline BoundaryOrg & $<-$ & Congruence & -0.5 & & & & -0.607 \\
\hline BoundaryPref & $<-$ & Congruence & 0.5 & & & & 0.693 \\
\hline Enhance_NtW_Aff_w1 & $<-$ & Congruence & -0.03 & 0.018 & -1.683 & 0.092 & -0.105 \\
\hline PCS_w2 & $<--$ & Congruence & 0.388 & 0.189 & 2.053 & 0.04 & 0.119 \\
\hline PLIW_w1 & $<--$ & Congruence & 0.013 & 0.016 & 0.787 & 0.431 & 0.05 \\
\hline Enhance_NtW_Aff_w1 & $<-$ & Education_w1 & 0.005 & 0.028 & 0.176 & 0.861 & 0.011 \\
\hline PCS_w2 & $<-$ & Education_w1 & 0.584 & 0.292 & 2.003 & 0.045 & 0.116 \\
\hline PLIW_w1 & $<--$ & Education_w1 & 0.02 & 0.026 & 0.802 & 0.422 & 0.051 \\
\hline Enhance_NtW_Aff1_w1 & $<-$ & Enhance_NtW_Aff_w1 & 1 & & & & 0.771 \\
\hline Enhance_NtW_Aff2_w1 & $<-$ & Enhance_NtW_Aff_w1 & 0.999 & 0.06 & 16.675 & $* * *$ & 0.874 \\
\hline Enhance_NtW_Aff3_w1 & $<-$ & Enhance_NtW_Aff_w1 & 1.022 & 0.059 & 17.406 & $* * *$ & 0.911 \\
\hline Enhance_NtW_Aff4_w1 & $<--$ & Enhance_NtW_Aff_w1 & 0.965 & 0.061 & 15.736 & $* * *$ & 0.833 \\
\hline PCS_w2 & $<-$ & Enhance_NtW_Aff_w1 & 1.294 & 0.65 & 1.99 & 0.047 & 0.114 \\
\hline Enhance_NtW_Aff_w1 & $<-$ & Income_w2 & 0.002 & 0.021 & 0.099 & 0.921 & 0.007 \\
\hline PCS_w2 & $<-$ & Income_w2 & -0.153 & 0.219 & -0.7 & 0.484 & -0.043 \\
\hline PLIW_w1 & $<-$ & Income_w2 & -0.006 & 0.019 & -0.297 & 0.767 & -0.02 \\
\hline Enhance_NtW_Aff_w1 & $<-$ & KidsAdult_w1 & -0.011 & 0.088 & -0.126 & 0.9 & -0.008 \\
\hline PCS_w2 & $<-$ & KidsAdult_w1 & -0.808 & 0.923 & -0.875 & 0.381 & -0.053 \\
\hline PLIW_w1 & $<-$ & KidsAdult_w1 & 0.055 & 0.081 & 0.676 & 0.499 & 0.045 \\
\hline BoundaryOrg & $<-$ & Level & 1 & & & & 0.717 \\
\hline BoundaryPref & $<-$ & Level & 1 & & & & 0.82 \\
\hline Enhance_NtW_Aff_w1 & $<-$ & Level & -0.02 & 0.03 & -0.643 & 0.52 & -0.04 \\
\hline PCS_w2 & $<-$ & Level & -0.55 & 0.32 & -1.72 & 0.085 & -0.1 \\
\hline PLIW_w1 & $<-$ & Level & -0.011 & 0.028 & -0.378 & 0.705 & -0.024 \\
\hline PCS_w2 & $<-$ & PLIW_w1 & -0.39 & 0.736 & -0.529 & 0.597 & -0.031 \\
\hline PLIW1_w1 & $<-$ & PLIW_w1 & 1 & & & & 0.648 \\
\hline PLIW2_w1 & $<-$ & PLIW_w1 & 1.138 & 0.095 & 12.02 & $* * *$ & 0.842 \\
\hline PLIW3_w1 & $<-$ & PLIW_w1 & 0.955 & 0.11 & 8.668 & $* * *$ & 0.557 \\
\hline PLIW4_w1 & $<--$ & PLIW_w1 & 1.156 & 0.099 & 11.679 & $* * *$ & 0.805 \\
\hline PLIW5_w1 & $<-$ & PLIW_w1 & 0.918 & 0.113 & 8.119 & $* * *$ & 0.517 \\
\hline PLIW6_w1 & $<-$ & PLIW_w1 & 1.027 & 0.091 & 11.316 & $* * *$ & 0.772 \\
\hline
\end{tabular}




\section{Model 18. PLIW, NtW Aff, MCS}

Nonwork-to-work conflict, nonwork-to-work affective enhancement, and mental health

Unstandardized Output:

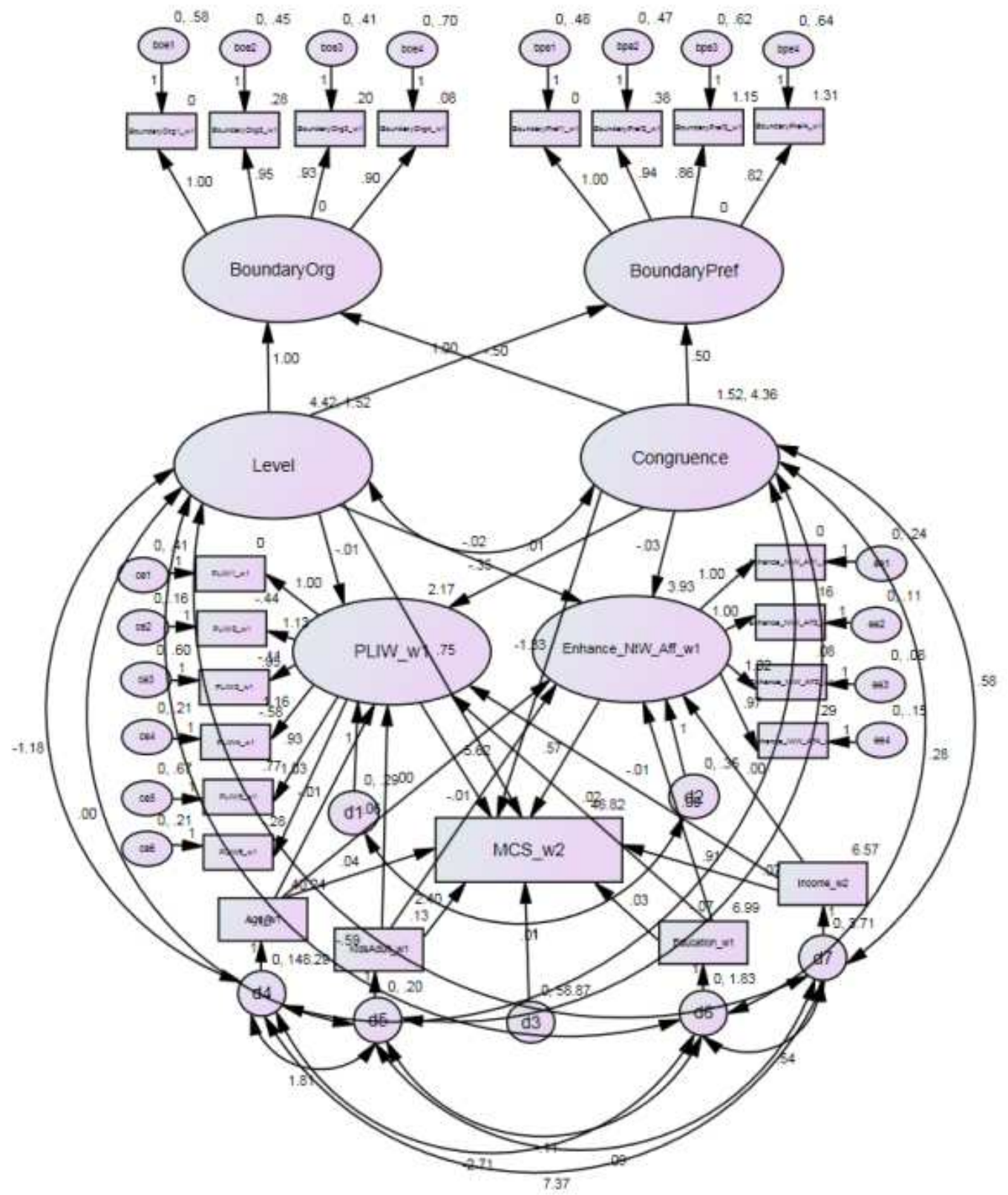

Fit Statistics:

- $\chi^{2}(199)=319.91, p<.001$

- CFI: 970

- RMSEA: .044 
Model 18 Output

\begin{tabular}{|c|c|c|c|c|c|c|c|}
\hline & & & Est. & S.E. & C.R. & $\mathrm{P}$ & $\begin{array}{l}\text { Std. } \\
\text { Est. }\end{array}$ \\
\hline Enhance_NtW_Aff_w1 & $<--$ & Age_w1 & 0.002 & 0.003 & 0.472 & 0.637 & 0.032 \\
\hline MCS_w2 & $<--$ & Age_w1 & 0.041 & 0.042 & 0.971 & 0.331 & 0.055 \\
\hline PLIW_w1 & $<--$ & Age_w1 & -0.006 & 0.003 & -1.969 & 0.049 & -0.136 \\
\hline BoundaryOrg1_w1 & $<--$ & BoundaryOrg & 1 & & & & 0.915 \\
\hline BoundaryOrg2_w1 & $<--$ & BoundaryOrg & 0.946 & 0.034 & 27.976 & $* * *$ & 0.925 \\
\hline BoundaryOrg3_w1 & $<--$ & BoundaryOrg & 0.931 & 0.033 & 28.267 & $* * *$ & 0.928 \\
\hline BoundaryOrg4_w1 & $<--$ & BoundaryOrg & 0.896 & 0.037 & 24.335 & $* * *$ & 0.879 \\
\hline BoundaryPref1_w1 & $<--$ & BoundaryPref & 1 & & & & 0.909 \\
\hline BoundaryPref2_w1 & $<--$ & BoundaryPref & 0.936 & 0.039 & 24.287 & $* * *$ & 0.9 \\
\hline BoundaryPref3_w1 & $<--$ & BoundaryPref & 0.857 & 0.04 & 21.645 & $* * *$ & 0.854 \\
\hline BoundaryPref4_w1 & $<--$ & BoundaryPref & 0.824 & 0.039 & 20.951 & $* * *$ & 0.841 \\
\hline BoundaryOrg & $<--$ & Congruence & -0.5 & & & & -0.607 \\
\hline BoundaryPref & $<--$ & Congruence & 0.5 & & & & 0.693 \\
\hline Enhance_NtW_Aff_w1 & $<--$ & Congruence & -0.03 & 0.018 & -1.676 & 0.094 & -0.105 \\
\hline MCS_w2 & $<--$ & Congruence & -1.335 & 0.227 & -5.889 & $* * *$ & -0.307 \\
\hline PLIW_w1 & $<--$ & Congruence & 0.013 & 0.016 & 0.794 & 0.427 & 0.05 \\
\hline Enhance_NtW_Aff_w1 & $<--$ & Education_w1 & 0.005 & 0.028 & 0.175 & 0.861 & 0.011 \\
\hline MCS_w2 & $<--$ & Education_w1 & 0.028 & 0.349 & 0.08 & 0.936 & 0.004 \\
\hline PLIW_w1 & $<--$ & Education_w1 & 0.021 & 0.026 & 0.825 & 0.41 & 0.053 \\
\hline Enhance_NtW_Aff1_w1 & $<--$ & Enhance_NtW_Aff_w1 & 1 & & & & 0.771 \\
\hline Enhance_NtW_Aff2_w1 & $<--$ & Enhance_NtW_Aff_w1 & 0.998 & 0.06 & 16.667 & $* * *$ & 0.873 \\
\hline Enhance_NtW_Aff3_w1 & $<--$ & Enhance_NtW_Aff_w1 & 1.023 & 0.059 & 17.413 & $* * *$ & 0.912 \\
\hline Enhance_NtW_Aff4_w1 & $<--$ & Enhance_NtW_Aff_w1 & 0.965 & 0.061 & 15.736 & $* * *$ & 0.833 \\
\hline MCS_w2 & $<--$ & Enhance_NtW_Aff_w1 & 0.573 & 0.774 & 0.741 & 0.459 & 0.038 \\
\hline Enhance_NtW_Aff_w1 & $<--$ & Income_w2 & 0.002 & 0.021 & 0.087 & 0.93 & 0.006 \\
\hline MCS_w2 & $<-$ & Income_w2 & 0.913 & 0.261 & 3.493 & $* * *$ & 0.193 \\
\hline PLIW_w1 & $<--$ & Income_w2 & -0.006 & 0.019 & -0.334 & 0.738 & -0.023 \\
\hline Enhance_NtW_Aff_w1 & $<--$ & KidsAdult_w1 & -0.013 & 0.088 & -0.151 & 0.88 & -0.01 \\
\hline MCS_w2 & $<--$ & KidsAdult_w1 & 2.398 & 1.102 & 2.176 & 0.03 & 0.117 \\
\hline PLIW_w1 & $<--$ & KidsAdult_w1 & 0.058 & 0.081 & 0.721 & 0.471 & 0.048 \\
\hline BoundaryOrg & $<--$ & Level & 1 & & & & 0.717 \\
\hline BoundaryPref & $<--$ & Level & 1 & & & & 0.82 \\
\hline Enhance_NtW_Aff_w1 & $<--$ & Level & -0.02 & 0.03 & -0.644 & 0.52 & -0.04 \\
\hline MCS_w2 & $<--$ & Level & 0.747 & 0.384 & 1.947 & 0.052 & 0.102 \\
\hline PLIW_w1 & $<--$ & Level & -0.011 & 0.028 & -0.388 & 0.698 & -0.025 \\
\hline MCS_w2 & $<--$ & PLIW_w1 & -5.618 & 0.961 & -5.844 & $* * *$ & -0.335 \\
\hline PLIW1_w1 & $<--$ & PLIW_w1 & 1 & & & & 0.648 \\
\hline PLIW2_w1 & $<--$ & PLIW_w1 & 1.133 & 0.094 & 12.001 & $* * *$ & 0.838 \\
\hline PLIW3_w1 & $<--$ & PLIW_w1 & 0.952 & 0.11 & 8.646 & $* * *$ & 0.555 \\
\hline PLIW4_w1 & $<--$ & PLIW_w1 & 1.163 & 0.099 & 11.732 & $* * *$ & 0.809 \\
\hline PLIW5_w1 & $<--$ & PLIW_w1 & 0.926 & 0.113 & 8.178 & $* * *$ & 0.521 \\
\hline PLIW6_w1 & $<--$ & PLIW_w1 & 1.029 & 0.091 & 11.329 & $* * *$ & 0.772 \\
\hline
\end{tabular}




\section{Model 19. WIPL, NtW Aff, MCS}

Work-to-nonwork conflict, nonwork-to-work affective enhancement, and mental health

\section{Unstandardized Output:}

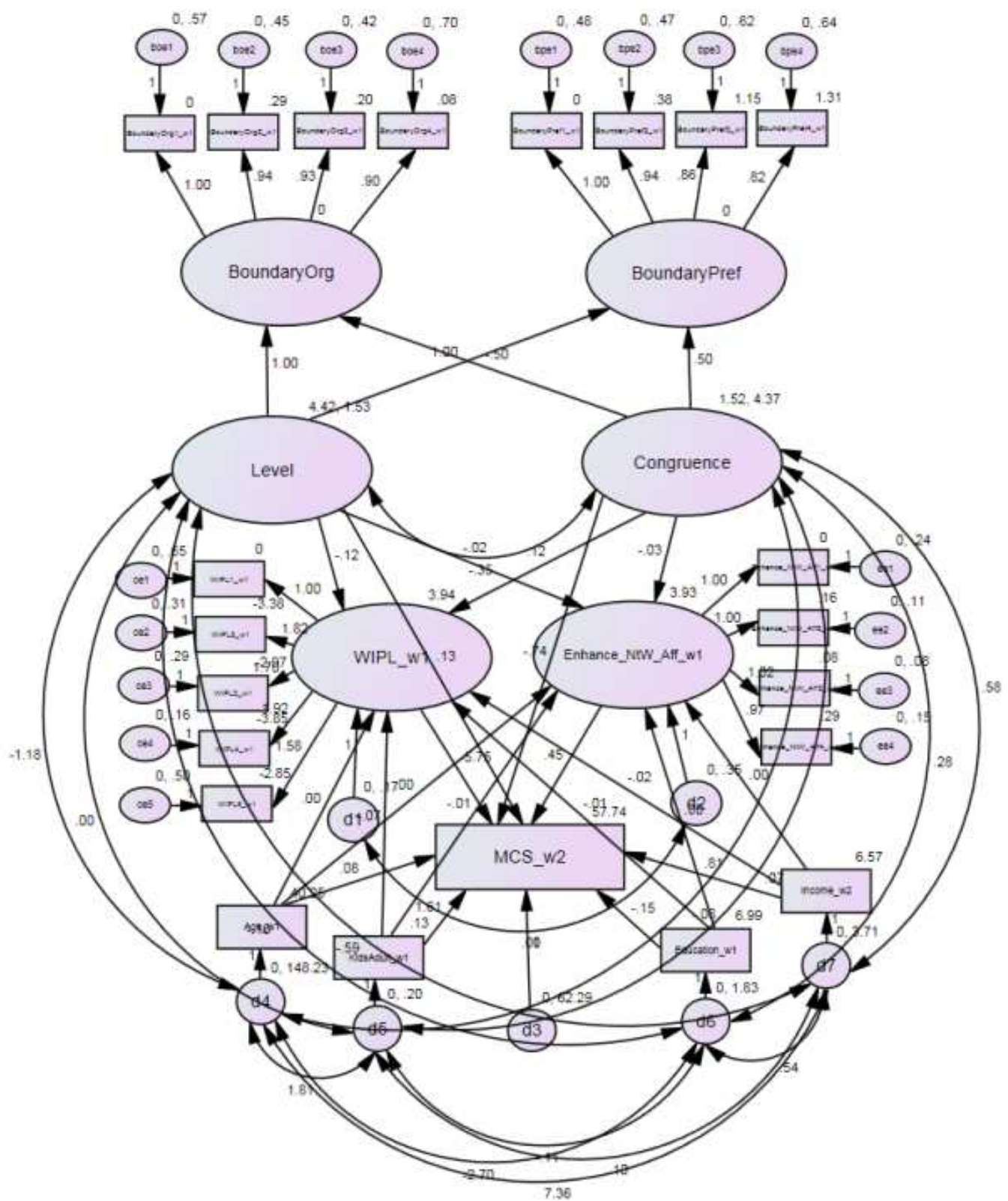

Fit Statistics:

- $\chi^{2}(178)=271.68, p<.001$

- CFI: .979

- RMSEA: .041 
Model 19 Output

\begin{tabular}{|c|c|c|c|c|c|c|c|}
\hline & & & Est. & S.E. & C.R. & $\mathrm{P}$ & Std. Est. \\
\hline Enhance_NtW_Aff_w1 & $<-$ & Age_w1 & 0.002 & 0.003 & 0.477 & 0.633 & 0.032 \\
\hline MCS_w2 & $<-$ & Age_w1 & 0.08 & 0.042 & 1.889 & 0.059 & 0.107 \\
\hline WIPL_w1 & $<-$ & Age_w1 & 0.001 & 0.002 & 0.319 & 0.749 & 0.018 \\
\hline BoundaryOrg1_w1 & $<-$ & BoundaryOrg & 1 & & & & 0.916 \\
\hline BoundaryOrg2_w1 & $<-$ & BoundaryOrg & 0.944 & 0.034 & 28.073 & $* * *$ & 0.924 \\
\hline BoundaryOrg3_w1 & $<-$ & BoundaryOrg & 0.929 & 0.033 & 28.339 & $* * *$ & 0.928 \\
\hline BoundaryOrg4_w1 & $<-$ & BoundaryOrg & 0.896 & 0.037 & 24.431 & $* * *$ & 0.879 \\
\hline BoundaryPref1_w1 & $<-$ & BoundaryPref & 1 & & & & 0.909 \\
\hline BoundaryPref2_w1 & $<-$ & BoundaryPref & 0.936 & 0.039 & 24.275 & $* * *$ & 0.9 \\
\hline BoundaryPref3_w1 & $<-$ & BoundaryPref & 0.857 & 0.04 & 21.661 & $* * *$ & 0.854 \\
\hline BoundaryPref4_w1 & $<-$ & BoundaryPref & 0.824 & 0.039 & 20.959 & $* * *$ & 0.841 \\
\hline BoundaryOrg & $<-$ & Congruence & -0.5 & & & & -0.607 \\
\hline BoundaryPref & $<-$ & Congruence & 0.5 & & & & 0.694 \\
\hline Enhance_NtW_Aff_w1 & $<-$ & Congruence & -0.03 & 0.018 & -1.671 & 0.095 & -0.104 \\
\hline MCS_w2 & $<-$ & Congruence & -0.743 & 0.271 & -2.743 & 0.006 & -0.171 \\
\hline WIPL_w1 & $<-$ & Congruence & 0.116 & 0.017 & 6.927 & $* * *$ & 0.481 \\
\hline Enhance_NtW_Aff_w1 & $<-$ & Education_w1 & 0.005 & 0.028 & 0.172 & 0.864 & 0.011 \\
\hline MCS_w2 & $<-$ & Education_w1 & -0.145 & 0.355 & -0.409 & 0.683 & -0.022 \\
\hline WIPL_w1 & $<-$ & Education_w1 & -0.01 & 0.02 & -0.483 & 0.629 & -0.026 \\
\hline Enhance_NtW_Aff1_w1 & $<-$ & Enhance_NtW_Aff_w1 & 1 & & & & 0.771 \\
\hline Enhance_NtW_Aff2_w1 & $<-$ & Enhance_NtW_Aff_w1 & 0.998 & 0.06 & 16.651 & $* * *$ & 0.873 \\
\hline Enhance_NtW_Aff3_w1 & $<-$ & Enhance_NtW_Aff_w1 & 1.024 & 0.059 & 17.408 & $* * *$ & 0.912 \\
\hline Enhance_NtW_Aff4_w1 & $<-$ & Enhance_NtW_Aff_w1 & 0.966 & 0.061 & 15.727 & $* * *$ & 0.833 \\
\hline MCS_w2 & $<-$ & Enhance_NtW_Aff_w1 & 0.452 & 0.788 & 0.573 & 0.567 & 0.03 \\
\hline Enhance_NtW_Aff_w1 & $<-$ & Income_w2 & 0.002 & 0.021 & 0.087 & 0.931 & 0.006 \\
\hline MCS_w2 & $<-$ & Income_w2 & 0.813 & 0.268 & 3.034 & 0.002 & 0.172 \\
\hline WIPL_w1 & $<-$ & Income_w2 & -0.024 & 0.015 & -1.579 & 0.114 & -0.09 \\
\hline Enhance_NtW_Aff_w1 & $<-$ & KidsAdult_w1 & -0.014 & 0.088 & -0.162 & 0.871 & -0.011 \\
\hline MCS_w2 & $<-$ & KidsAdult_w1 & 1.615 & 1.126 & 1.434 & 0.152 & 0.079 \\
\hline WIPL_w1 & $<-$ & KidsAdult_w1 & -0.069 & 0.063 & -1.103 & 0.27 & -0.061 \\
\hline BoundaryOrg & $<-$ & Level & 1 & & & & 0.717 \\
\hline BoundaryPref & $<-$ & Level & 1 & & & & 0.82 \\
\hline Enhance_NtW_Aff_w1 & $<-$ & Level & -0.02 & 0.03 & -0.644 & 0.52 & -0.04 \\
\hline MCS_w2 & $<-$ & Level & 0.133 & 0.414 & 0.321 & 0.748 & 0.018 \\
\hline WIPL_w1 & $<-$ & Level & -0.117 & 0.024 & -4.781 & $* * *$ & -0.287 \\
\hline MCS_w2 & $<-$ & WIPL_w1 & -5.749 & 1.283 & -4.482 & $* * *$ & -0.319 \\
\hline WIPL1_w1 & $<-$ & WIPL_w1 & 1 & & & & 0.529 \\
\hline WIPL2_w1 & $<-$ & WIPL_w1 & 1.824 & 0.184 & 9.91 & $* * *$ & 0.854 \\
\hline WIPL3_w1 & $<-$ & WIPL_w1 & 1.705 & 0.173 & 9.872 & $* * *$ & 0.847 \\
\hline WIPL4_w1 & $<-$ & WIPL_W1 & 1.916 & 0.187 & 10.223 & $* * *$ & 0.923 \\
\hline WIPL5_w1 & $<-$ & WIPL_w1 & 1.585 & 0.171 & 9.291 & $* * *$ & 0.75 \\
\hline
\end{tabular}




\section{Model 20. WIPL, NtW Aff, PCS}

Work-to-nonwork conflict, nonwork-to-work affective enhancement, and physical health

\section{Unstandardized Output:}

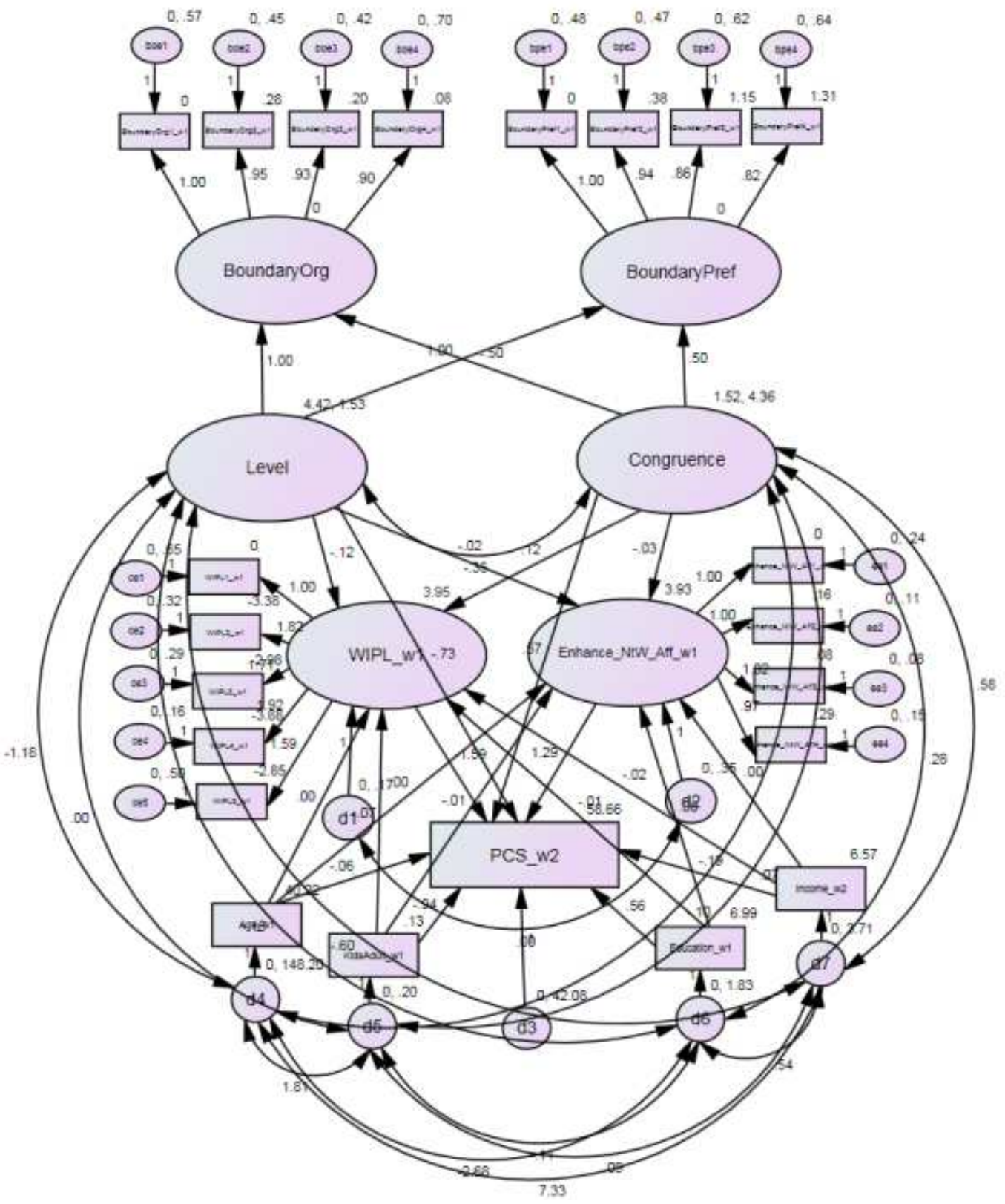

Fit Statistics:

- $\chi^{2}(178)=262.79, p<.001$

- $\quad$ CFI: .981

- RMSEA: .039 


\begin{tabular}{|c|c|c|c|c|c|c|c|}
\hline & & & Est. & S.E. & C.R. & $\mathrm{p}$ & Std. Est. \\
\hline Enhance_NtW_Aff_w1 & $<-$ & Age_w1 & 0.002 & 0.003 & 0.473 & 0.636 & 0.032 \\
\hline PCS_w2 & $<-$ & Age_w1 & -0.064 & 0.035 & -1.854 & 0.064 & -0.115 \\
\hline WIPL_w1 & $<-$ & Age_w1 & 0.001 & 0.002 & 0.304 & 0.761 & 0.017 \\
\hline BoundaryOrg1_w1 & $<-$ & BoundaryOrg & 1 & & & & 0.916 \\
\hline BoundaryOrg2_w1 & $<-$ & BoundaryOrg & 0.945 & 0.034 & 28.079 & $* * *$ & 0.925 \\
\hline BoundaryOrg3_w1 & $<-$ & BoundaryOrg & 0.93 & 0.033 & 28.327 & $* * *$ & 0.928 \\
\hline BoundaryOrg4_w1 & $<-$ & BoundaryOrg & 0.896 & 0.037 & 24.398 & $* * *$ & 0.879 \\
\hline BoundaryPref1_w1 & $<-$ & BoundaryPref & 1 & & & & 0.909 \\
\hline BoundaryPref2_w1 & $<-$ & BoundaryPref & 0.937 & 0.039 & 24.276 & $* * *$ & 0.9 \\
\hline BoundaryPref3_w1 & $<-$ & BoundaryPref & 0.857 & 0.04 & 21.638 & $* * *$ & 0.854 \\
\hline BoundaryPref4_w1 & $<-$ & BoundaryPref & 0.824 & 0.039 & 20.952 & $* * *$ & 0.841 \\
\hline BoundaryOrg & $<-$ & Congruence & -0.5 & & & & -0.607 \\
\hline BoundaryPref & $<-$ & Congruence & 0.5 & & & & 0.694 \\
\hline Enhance_NtW_Aff_w1 & $<-$ & Congruence & -0.03 & 0.018 & -1.68 & 0.093 & -0.105 \\
\hline PCS_w2 & $<-$ & Congruence & 0.566 & 0.222 & 2.554 & 0.011 & 0.174 \\
\hline WIPL_w1 & $<-$ & Congruence & 0.116 & 0.017 & 6.92 & $* * *$ & 0.481 \\
\hline Enhance_NtW_Aff_w1 & $<-$ & Education_w1 & 0.005 & 0.028 & 0.177 & 0.859 & 0.011 \\
\hline PCS_w2 & $<-$ & Education_w1 & 0.562 & 0.29 & 1.934 & 0.053 & 0.112 \\
\hline WIPL_w1 & $<-$ & Education_w1 & -0.01 & 0.02 & -0.492 & 0.622 & -0.026 \\
\hline Enhance_NtW_Aff1_w1 & $<-$ & Enhance_NtW_Aff_w1 & 1 & & & & 0.771 \\
\hline Enhance_NtW_Aff2_w1 & $<-$ & Enhance_NtW_Aff_w1 & 0.999 & 0.06 & 16.663 & $* * *$ & 0.873 \\
\hline Enhance_NtW_Aff3_w1 & $<-$ & Enhance_NtW_Aff_w1 & 1.023 & 0.059 & 17.403 & $* * *$ & 0.912 \\
\hline Enhance_NtW_Aff4_w1 & $<-$ & Enhance_NtW_Aff_w1 & 0.965 & 0.061 & 15.73 & $* * *$ & 0.833 \\
\hline PCS_w2 & $<-$ & Enhance_NtW_Aff_w1 & 1.287 & 0.648 & 1.987 & 0.047 & 0.113 \\
\hline Enhance_NtW_Aff_w1 & $<-$ & Income_w2 & 0.002 & 0.021 & 0.101 & 0.92 & 0.007 \\
\hline PCS_w2 & $<-$ & Income_w2 & -0.189 & 0.219 & -0.862 & 0.389 & -0.054 \\
\hline WIPL_w1 & $<-$ & Income_w2 & -0.024 & 0.015 & -1.59 & 0.112 & -0.091 \\
\hline Enhance_NtW_Aff_w1 & $<-$ & KidsAdult_w1 & -0.01 & 0.088 & -0.111 & 0.912 & -0.007 \\
\hline PCS_w2 & $<-$ & KidsAdult_w1 & -0.942 & 0.922 & -1.022 & 0.307 & -0.061 \\
\hline WIPL_w1 & $<-$ & KidsAdult_w1 & -0.069 & 0.063 & -1.106 & 0.269 & -0.061 \\
\hline BoundaryOrg & $<-$ & Level & 1 & & & & 0.717 \\
\hline BoundaryPref & $<-$ & Level & 1 & & & & 0.82 \\
\hline Enhance_NtW_Aff_w1 & $<-$ & Level & -0.02 & 0.03 & -0.643 & 0.52 & -0.04 \\
\hline PCS_w2 & $<-$ & Level & -0.731 & 0.339 & -2.157 & 0.031 & -0.133 \\
\hline WIPL_w1 & $<-$ & Level & -0.117 & 0.024 & -4.788 & $* * *$ & -0.288 \\
\hline PCS_w2 & $<-$ & WIPL_w1 & -1.589 & 0.967 & -1.642 & 0.1 & -0.118 \\
\hline WIPL1_w1 & $<-$ & WIPL_w1 & 1 & & & & 0.528 \\
\hline WIPL2_w1 & $<-$ & WIPL_w1 & 1.823 & 0.185 & 9.879 & $* * *$ & 0.852 \\
\hline WIPL3_w1 & $<-$ & WIPL_w1 & 1.707 & 0.173 & 9.849 & $* * *$ & 0.846 \\
\hline WIPL4_w1 & $<-$ & WIPL_w1 & 1.925 & 0.189 & 10.209 & $* * *$ & 0.926 \\
\hline WIPL5_w1 & $<-$ & WIPL_w1 & 1.585 & 0.171 & 9.266 & $* * *$ & 0.748 \\
\hline
\end{tabular}




\section{Model 21. WIPL, NtW Val, PCS}

Work-to-nonwork conflict, nonwork-to-work value-based enhancement, and physical health

\section{Unstandardized output:}

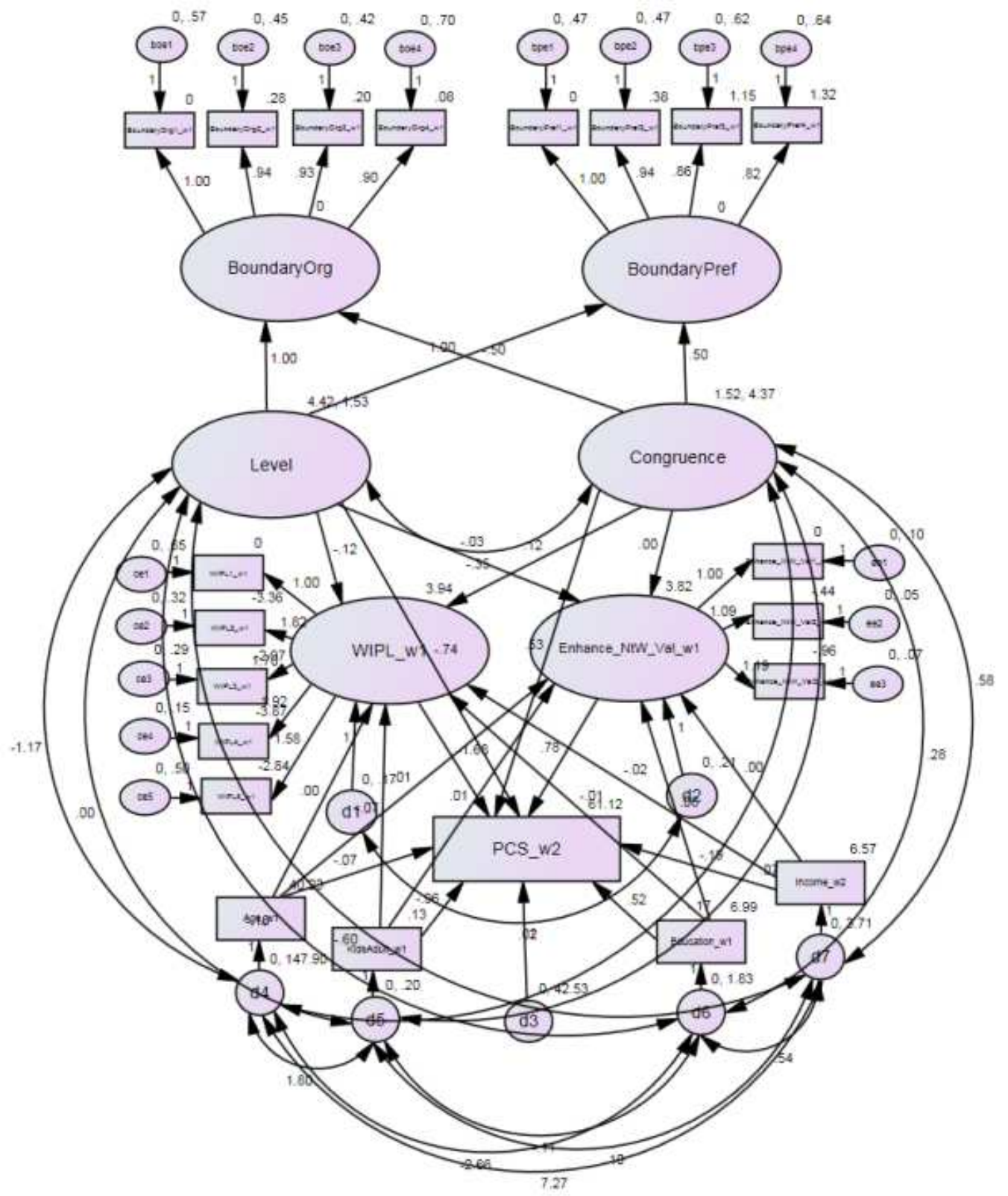

Fit Statistics:

- $\chi^{2}(158)=245.10, p<.001$

- CFI: 980

- RMSEA: .042 
Model 21 Output

\begin{tabular}{|c|c|c|c|c|c|c|c|}
\hline & & & Est. & S.E. & C.R. & $\mathrm{P}$ & Std. Est. \\
\hline Enhance_NtW_Val_w1 & $<--$ & Age_w1 & 0.011 & 0.003 & 4.403 & $* * *$ & 0.285 \\
\hline PCS_w2 & $<--$ & Age_w1 & -0.072 & 0.036 & -1.979 & 0.048 & -0.128 \\
\hline WIPL_w1 & $<-$ & Age_w1 & 0.001 & 0.002 & 0.363 & 0.717 & 0.021 \\
\hline BoundaryOrg1_w1 & $<-$ & BoundaryOrg & 1 & & & & 0.916 \\
\hline BoundaryOrg2_w1 & $<--$ & BoundaryOrg & 0.945 & 0.034 & 28.084 & $* * *$ & 0.924 \\
\hline BoundaryOrg3_w1 & $<--$ & BoundaryOrg & 0.93 & 0.033 & 28.328 & $* * *$ & 0.928 \\
\hline BoundaryOrg4_w1 & $<-$ & BoundaryOrg & 0.896 & 0.037 & 24.42 & $* * *$ & 0.879 \\
\hline BoundaryPref1_w1 & $<--$ & BoundaryPref & 1 & & & & 0.909 \\
\hline BoundaryPref2_w1 & $<-$ & BoundaryPref & 0.936 & 0.039 & 24.282 & $* * *$ & 0.9 \\
\hline BoundaryPref3_w1 & $<--$ & BoundaryPref & 0.857 & 0.04 & 21.668 & $* * *$ & 0.854 \\
\hline BoundaryPref4_w1 & $<--$ & BoundaryPref & 0.823 & 0.039 & 20.943 & $* * *$ & 0.84 \\
\hline BoundaryOrg & $<-$ & Congruence & -0.5 & & & & -0.607 \\
\hline BoundaryPref & $<-$ & Congruence & 0.5 & & & & 0.694 \\
\hline Enhance_NtW_Val_w1 & $<--$ & Congruence & 0.003 & 0.014 & 0.232 & 0.816 & 0.014 \\
\hline PCS_w2 & $<-$ & Congruence & 0.535 & 0.222 & 2.408 & 0.016 & 0.164 \\
\hline WIPL_w1 & $<-$ & Congruence & 0.116 & 0.017 & 6.93 & $* * *$ & 0.481 \\
\hline Enhance_NtW_Val_w1 & $<-$ & Education_w1 & 0.055 & 0.021 & 2.567 & 0.01 & 0.154 \\
\hline PCS_w2 & $<-$ & Education_w1 & 0.525 & 0.296 & 1.775 & 0.076 & 0.105 \\
\hline WIPL_w1 & $<-$ & Education_w1 & -0.01 & 0.02 & -0.486 & 0.627 & -0.026 \\
\hline Enhance_NtW_Val1_w1 & $<--$ & Enhance_NtW_Val_w1 & 1 & & & & 0.841 \\
\hline Enhance_NtW_Val2_w1 & $<-$ & Enhance_NtW_Val_w1 & 1.091 & 0.053 & 20.703 & $* * *$ & 0.917 \\
\hline Enhance_NtW_Val3_w1 & $<-$ & Enhance_NtW_Val_w1 & 1.195 & 0.058 & 20.513 & $* * *$ & 0.909 \\
\hline PCS_w2 & $<--$ & Enhance_NtW_Val_w1 & 0.78 & 0.851 & 0.916 & 0.36 & 0.056 \\
\hline Enhance_NtW_Val_w1 & $<--$ & Income_w2 & 0.001 & 0.016 & 0.047 & 0.963 & 0.003 \\
\hline PCS_w2 & $<-$ & Income_w2 & -0.19 & 0.22 & -0.861 & 0.389 & -0.054 \\
\hline WIPL_w1 & $<--$ & Income_w2 & -0.024 & 0.015 & -1.606 & 0.108 & -0.092 \\
\hline Enhance_NtW_Val_w1 & $<-$ & KidsAdult_w1 & 0.012 & 0.068 & 0.182 & 0.856 & 0.011 \\
\hline PCS_w2 & $<-$ & KidsAdult_w1 & -0.964 & 0.927 & -1.04 & 0.298 & -0.063 \\
\hline WIPL_w1 & $<-$ & KidsAdult_w1 & -0.07 & 0.063 & -1.12 & 0.263 & -0.062 \\
\hline BoundaryOrg & $<--$ & Level & 1 & & & & 0.717 \\
\hline BoundaryPref & $<--$ & Level & 1 & & & & 0.82 \\
\hline Enhance_NtW_Val_w1 & $<--$ & Level & -0.034 & 0.023 & -1.45 & 0.147 & -0.087 \\
\hline PCS_w2 & $<-$ & Level & -0.741 & 0.341 & -2.175 & 0.03 & -0.135 \\
\hline WIPL_w1 & $<-$ & Level & -0.117 & 0.024 & -4.79 & $* * *$ & -0.288 \\
\hline PCS_w2 & $<--$ & WIPL_w1 & -1.68 & 0.979 & -1.716 & 0.086 & -0.125 \\
\hline WIPL1_w1 & & WIPL_w1 & 1 & & & & 0.529 \\
\hline WIPL2_w1 & $<-$ & WIPL_w1 & 1.818 & 0.184 & 9.897 & $* * *$ & 0.851 \\
\hline WIPL3_w1 & $<-$ & WIPL_w1 & 1.704 & 0.173 & 9.871 & $* * *$ & 0.846 \\
\hline WIPL4_w1 & $<--$ & WIPL_w1 & 1.923 & 0.188 & 10.236 & $* * *$ & 0.927 \\
\hline WIPL5_w1 & $<--$ & WIPL_w1 & 1.581 & 0.17 & 9.28 & $* * *$ & 0.748 \\
\hline
\end{tabular}




\section{Model 22. WIPL, NtW Val, MCS}

Work-to-nonwork conflict, nonwork-to-work value-based enhancement, and mental health

\section{Unstandardized Output:}

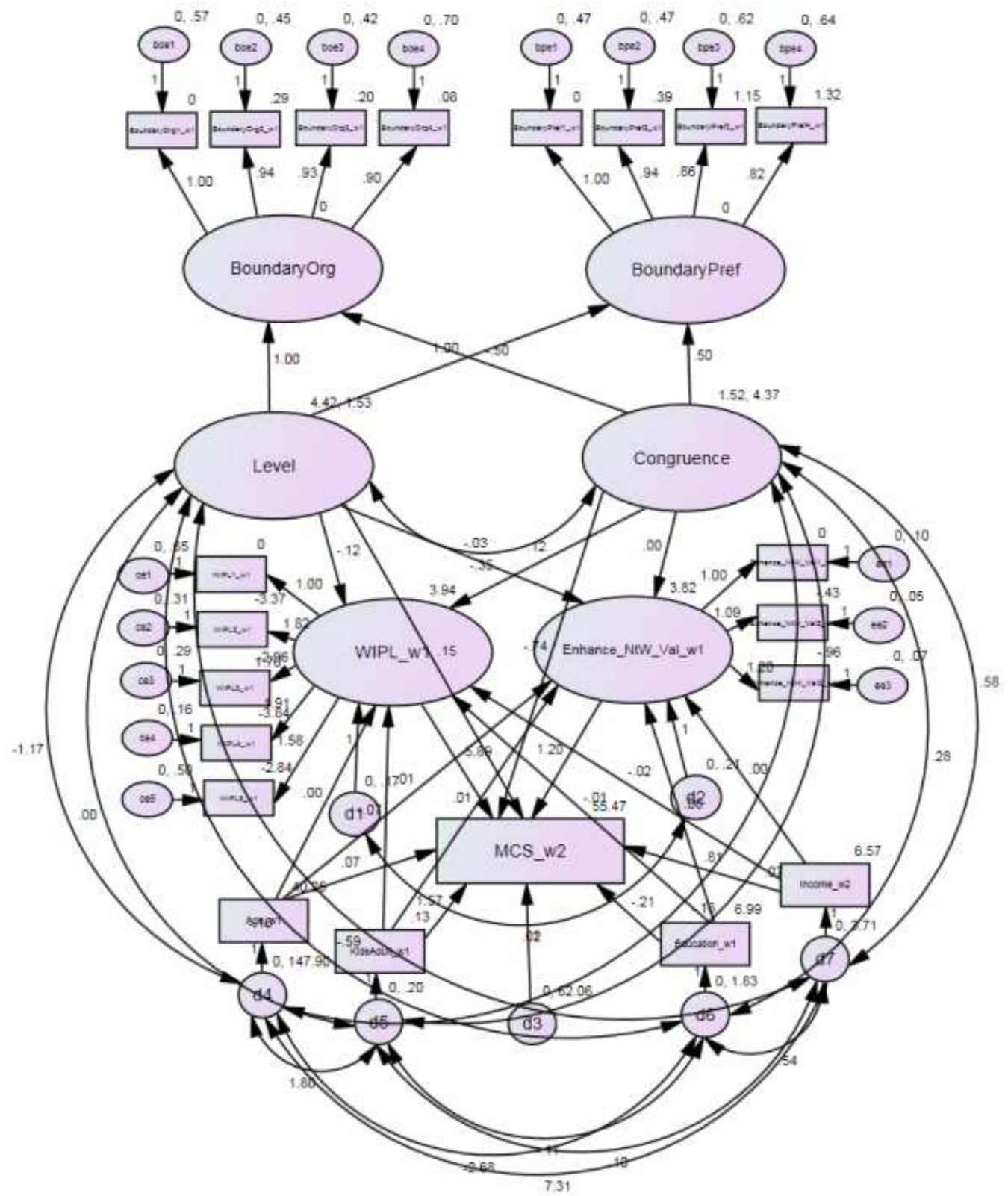

Fit Statistics:

- $\chi^{2}(158)=243.97, p<.001$

- CFI: 980

- RMSEA: .042 
Model 22 Output

\begin{tabular}{|c|c|c|c|c|c|c|c|}
\hline & & & Est. & S.E. & C.R. & $\mathrm{P}$ & $\begin{array}{l}\text { Std. } \\
\text { Est. }\end{array}$ \\
\hline Enhance_NtW_Val_w1 & $<-$ & Age_w1 & 0.011 & 0.003 & 4.4 & $* * *$ & 0.285 \\
\hline MCS_w2 & $<-$ & Age_w1 & 0.068 & 0.044 & 1.543 & 0.123 & 0.091 \\
\hline WIPL_w1 & $<-$ & Age_w1 & 0.001 & 0.002 & 0.371 & 0.71 & 0.021 \\
\hline BoundaryOrg1_w1 & $<-$ & BoundaryOrg & 1 & & & & 0.916 \\
\hline BoundaryOrg2_w1 & $<-$ & BoundaryOrg & 0.944 & 0.034 & 28.075 & $* * *$ & 0.924 \\
\hline BoundaryOrg3_w1 & $<-$ & BoundaryOrg & 0.929 & 0.033 & 28.341 & $* * *$ & 0.927 \\
\hline BoundaryOrg4_w1 & $<-$ & BoundaryOrg & 0.896 & 0.037 & 24.451 & $* * *$ & 0.879 \\
\hline BoundaryPref1_w1 & $<-$ & BoundaryPref & 1 & & & & 0.91 \\
\hline BoundaryPref2_w1 & $<-$ & BoundaryPref & 0.935 & 0.039 & 24.282 & $* * *$ & 0.899 \\
\hline BoundaryPref3_w1 & $<-$ & BoundaryPref & 0.857 & 0.04 & 21.687 & $* * *$ & 0.854 \\
\hline BoundaryPref4_w1 & $<-$ & BoundaryPref & 0.823 & 0.039 & 20.953 & $* * *$ & 0.84 \\
\hline BoundaryOrg & $<-$ & Congruence & -0.5 & & & & -0.607 \\
\hline BoundaryPref & $<-$ & Congruence & 0.5 & & & & 0.694 \\
\hline Enhance_NtW_Val_w1 & $<-$ & Congruence & 0.003 & 0.014 & 0.234 & 0.815 & 0.014 \\
\hline MCS_w2 & $<-$ & Congruence & -0.741 & 0.27 & -2.747 & 0.006 & -0.17 \\
\hline WIPL_w1 & $<-$ & Congruence & 0.116 & 0.017 & 6.938 & $* * *$ & 0.482 \\
\hline Enhance_NtW_Val_w1 & $<-$ & Education_w1 & 0.055 & 0.021 & 2.576 & 0.01 & 0.155 \\
\hline MCS_w2 & $<-$ & Education_w1 & -0.213 & 0.359 & -0.592 & 0.554 & -0.032 \\
\hline WIPL_w1 & $<-$ & Education_w1 & -0.009 & 0.02 & -0.478 & 0.633 & -0.025 \\
\hline Enhance_NtW_Val1_w1 & $<-$ & Enhance_NtW_Val_w1 & 1 & & & & 0.841 \\
\hline Enhance_NtW_Val2_w1 & $<-$ & Enhance_NtW_Val_w1 & 1.089 & 0.053 & 20.699 & $* * *$ & 0.916 \\
\hline Enhance_NtW_Val3_w1 & $<-$ & Enhance_NtW_Val_w1 & 1.195 & 0.058 & 20.554 & $* * *$ & 0.91 \\
\hline MCS_w2 & $<-$ & Enhance_NtW_Val_w1 & 1.204 & 1.033 & 1.165 & 0.244 & 0.064 \\
\hline Enhance_NtW_Val_w1 & $<-$ & Income_w2 & 0 & 0.016 & 0.031 & 0.975 & 0.002 \\
\hline MCS_w2 & $<-$ & Income_w2 & 0.81 & 0.267 & 3.029 & 0.002 & 0.172 \\
\hline WIPL_w1 & $<-$ & Income_w2 & -0.024 & 0.015 & -1.594 & 0.111 & -0.091 \\
\hline Enhance_NtW_Val_w1 & $<-$ & KidsAdult_w1 & 0.012 & 0.068 & 0.181 & 0.857 & 0.011 \\
\hline MCS_w2 & $<-$ & KidsAdult_w1 & 1.571 & 1.125 & 1.397 & 0.162 & 0.076 \\
\hline WIPL_w1 & $<-$ & KidsAdult_w1 & -0.07 & 0.063 & -1.121 & 0.262 & -0.062 \\
\hline BoundaryOrg & $<-$ & Level & 1 & & & & 0.717 \\
\hline BoundaryPref & $<-$ & Level & 1 & & & & 0.82 \\
\hline Enhance_NtW_Val_w1 & $<-$ & Level & -0.034 & 0.023 & -1.457 & 0.145 & -0.087 \\
\hline MCS_w2 & $<-$ & Level & 0.147 & 0.413 & 0.356 & 0.722 & 0.02 \\
\hline WIPL_w1 & $<-$ & Level & -0.117 & 0.024 & -4.786 & $* * *$ & -0.287 \\
\hline MCS_w2 & $<-$ & WIPL_w1 & -5.891 & 1.292 & -4.559 & $* * *$ & -0.327 \\
\hline WIPL1_w1 & $<-$ & WIPL_w1 & 1 & & & & 0.53 \\
\hline WIPL2_w1 & $<-$ & WIPL_w1 & 1.819 & 0.183 & 9.932 & $* * *$ & 0.853 \\
\hline WIPL3_w1 & $<-$ & WIPL_w1 & 1.702 & 0.172 & 9.898 & $* * *$ & 0.847 \\
\hline WIPL4_w1 & $<-$ & WIPL_w1 & 1.913 & 0.187 & 10.254 & $* * *$ & 0.924 \\
\hline WIPL5_w1 & $<-$ & WIPL_w1 & 1.58 & 0.17 & 9.308 & $* * *$ & 0.749 \\
\hline
\end{tabular}




\section{Model 23. PLIW, NtW Val, MCS}

Nonwork-to-work conflict, nonwork-to-work value-based enhancement, and mental health

\section{Unstandardized Output:}

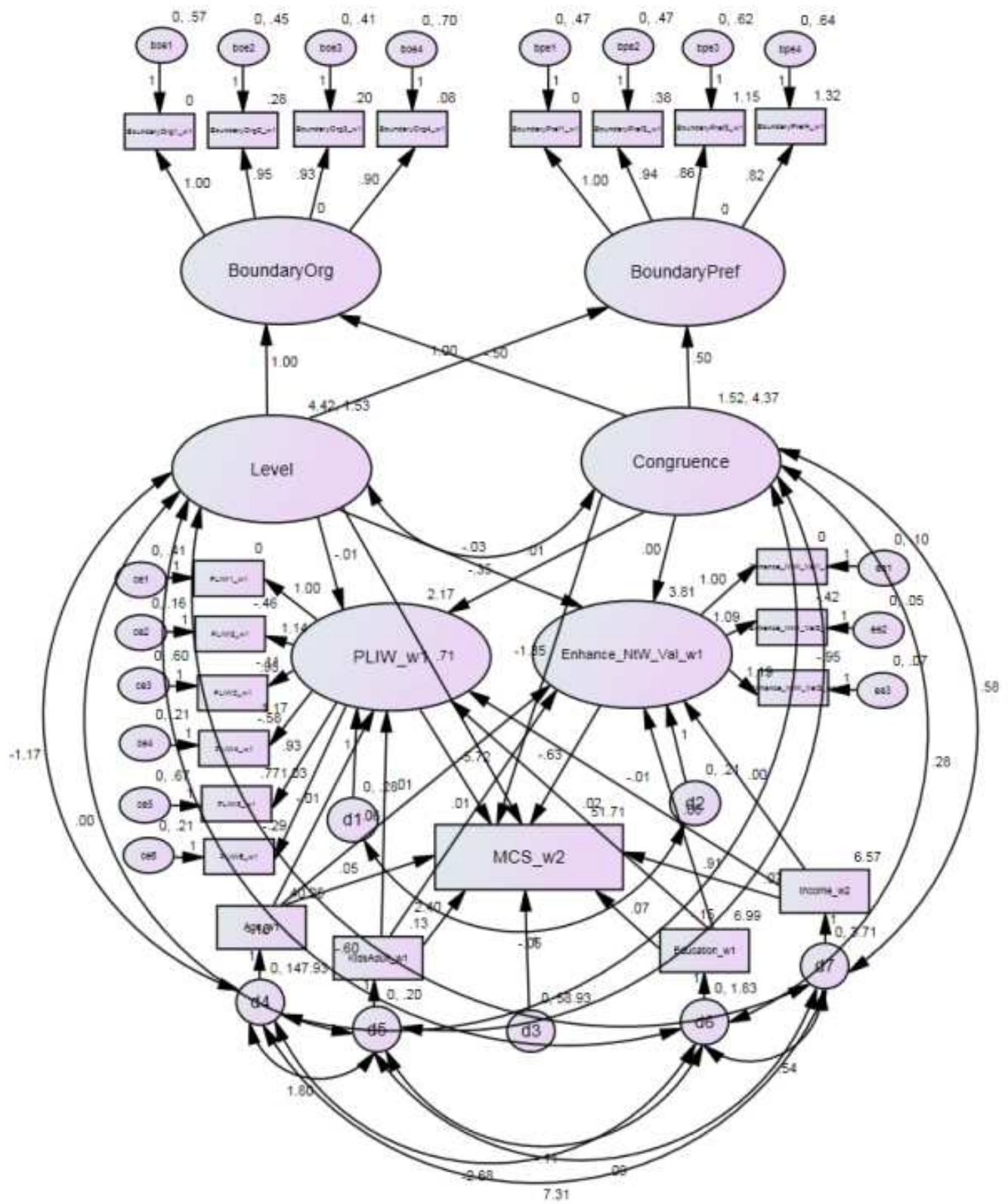

Fit Statistics:

- $\chi^{2}(178)=297.31, p<.001$

- CFI: .970

- RMSEA: .046 
Model 23 Output

\begin{tabular}{|c|c|c|c|c|c|c|c|}
\hline & & & Est. & S.E. & C.R. & $\mathrm{P}$ & $\begin{array}{l}\text { Std. } \\
\text { Est. }\end{array}$ \\
\hline Enhance_NtW_Val_w1 & $<--$ & Age_w1 & 0.011 & 0.003 & 4.404 & $* * *$ & 0.286 \\
\hline MCS_w2 & $<--$ & Age_w1 & 0.048 & 0.043 & 1.119 & 0.263 & 0.065 \\
\hline PLIW_w1 & $<--$ & Age_w1 & -0.006 & 0.003 & -1.97 & 0.049 & -0.136 \\
\hline BoundaryOrg1_w1 & $<--$ & BoundaryOrg & 1 & & & & 0.915 \\
\hline BoundaryOrg2_w1 & $<--$ & BoundaryOrg & 0.945 & 0.034 & 27.974 & $* * *$ & 0.924 \\
\hline BoundaryOrg3_w1 & $<--$ & BoundaryOrg & 0.931 & 0.033 & 28.277 & $* * *$ & 0.928 \\
\hline BoundaryOrg4_w1 & $<--$ & BoundaryOrg & 0.896 & 0.037 & 24.375 & $* * *$ & 0.879 \\
\hline BoundaryPref1_w1 & $<--$ & BoundaryPref & 1 & & & & 0.91 \\
\hline BoundaryPref2_w1 & $<--$ & BoundaryPref & 0.936 & 0.039 & 24.289 & $* * *$ & 0.9 \\
\hline BoundaryPref3_w1 & $<--$ & BoundaryPref & 0.857 & 0.04 & 21.668 & $* * *$ & 0.854 \\
\hline BoundaryPref4_w1 & $<--$ & BoundaryPref & 0.823 & 0.039 & 20.947 & $* * *$ & 0.84 \\
\hline BoundaryOrg & $<--$ & Congruence & -0.5 & & & & -0.607 \\
\hline BoundaryPref & $<--$ & Congruence & 0.5 & & & & 0.693 \\
\hline Enhance_NtW_Val_w1 & $<--$ & Congruence & 0.003 & 0.014 & 0.24 & 0.811 & 0.014 \\
\hline MCS_w2 & $<--$ & Congruence & -1.347 & 0.225 & -5.976 & $* * *$ & -0.31 \\
\hline PLIW_w1 & $<--$ & Congruence & 0.013 & 0.016 & 0.79 & 0.43 & 0.05 \\
\hline Enhance_NtW_Val_w1 & $<--$ & Education_w1 & 0.055 & 0.022 & 2.564 & 0.01 & 0.154 \\
\hline MCS_w2 & $<--$ & Education_w1 & 0.066 & 0.354 & 0.185 & 0.853 & 0.01 \\
\hline PLIW_w1 & $<--$ & Education_w1 & 0.021 & 0.025 & 0.825 & 0.409 & 0.053 \\
\hline Enhance_NtW_Val1_w1 & $<--$ & Enhance_NtW_Val_w1 & 1 & & & & 0.843 \\
\hline Enhance_NtW_Val2_w1 & $<--$ & Enhance_NtW_Val_w1 & 1.087 & 0.052 & 20.753 & $* * *$ & 0.915 \\
\hline Enhance_NtW_Val3_w1 & $<--$ & Enhance_NtW_Val_w1 & 1.193 & 0.058 & 20.613 & $* * *$ & 0.909 \\
\hline MCS_w2 & $<--$ & Enhance_NtW_Val_w1 & -0.634 & 1.027 & -0.617 & 0.537 & -0.034 \\
\hline Enhance_NtW_Val_w1 & $<--$ & Income_w2 & 0.001 & 0.016 & 0.05 & 0.96 & 0.003 \\
\hline MCS_w2 & $<--$ & Income_w2 & 0.914 & 0.261 & 3.501 & $* * *$ & 0.194 \\
\hline PLIW_w1 & $<--$ & Income_w2 & -0.006 & 0.019 & -0.337 & 0.736 & -0.023 \\
\hline Enhance_NtW_Val_w1 & $<--$ & KidsAdult_w1 & 0.01 & 0.068 & 0.144 & 0.885 & 0.009 \\
\hline MCS_w2 & $<--$ & KidsAdult_w1 & 2.398 & 1.103 & 2.175 & 0.03 & 0.117 \\
\hline PLIW_w1 & $<--$ & KidsAdult_w1 & 0.059 & 0.08 & 0.731 & 0.465 & 0.048 \\
\hline BoundaryOrg & $<--$ & Level & 1 & & & & 0.717 \\
\hline BoundaryPref & $<--$ & Level & 1 & & & & 0.82 \\
\hline Enhance_NtW_Val_w1 & $<--$ & Level & -0.034 & 0.024 & -1.445 & 0.149 & -0.086 \\
\hline MCS_w2 & $<--$ & Level & 0.714 & 0.386 & 1.851 & 0.064 & 0.097 \\
\hline PLIW_w1 & $<--$ & Level & -0.011 & 0.028 & -0.387 & 0.699 & -0.025 \\
\hline MCS_w2 & $<--$ & PLIW_w1 & -5.721 & 0.986 & -5.801 & $* * *$ & -0.339 \\
\hline PLIW1_w1 & $<--$ & PLIW_w1 & 1 & & & & 0.645 \\
\hline PLIW2_w1 & $<--$ & PLIW_w1 & 1.141 & 0.095 & 11.98 & $* * *$ & 0.841 \\
\hline PLIW3_w1 & $<--$ & PLIW_w1 & 0.95 & 0.111 & 8.588 & $* * *$ & 0.552 \\
\hline PLIW4_w1 & $<--$ & PLIW_w1 & 1.167 & 0.1 & 11.685 & $* * *$ & 0.809 \\
\hline PLIW5_w1 & $<--$ & PLIW_w1 & 0.927 & 0.114 & 8.143 & $* * *$ & 0.52 \\
\hline PLIW6_w1 & $<--$ & PLIW_w1 & 1.035 & 0.092 & 11.305 & $* * *$ & 0.774 \\
\hline
\end{tabular}




\section{Model 24. PLIW, NtW Val, PCS}

Nonwork-to-work conflict, nonwork-to-work value-based enhancement, and physical health

\section{Unstandardized Output:}

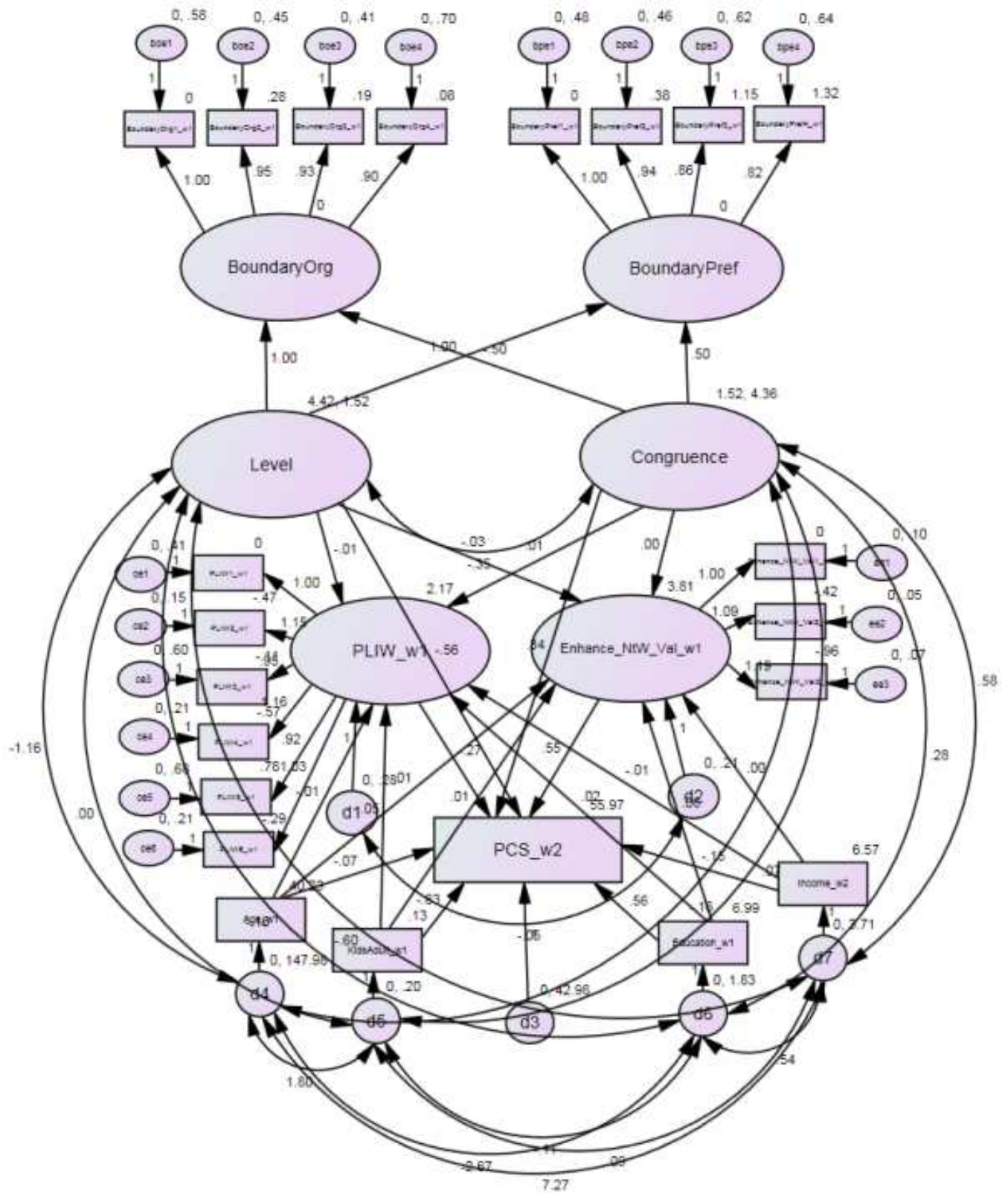

Fit Statistics:

- $\chi^{2}(178)=294.35, p<.001$

- $\quad$ CFI: .971

- RMSEA: .046 
Model 24 Output

\begin{tabular}{|c|c|c|c|c|c|c|c|}
\hline & & & Est. & S.E. & C.R. & $\mathrm{P}$ & $\begin{array}{l}\text { Std. } \\
\text { Est. }\end{array}$ \\
\hline Enhance_NtW_Val_w1 & $<-$ & Age_w1 & 0.011 & 0.003 & 4.405 & $* * *$ & 0.285 \\
\hline PCS_w2 & $<-$ & Age_w1 & -0.072 & 0.036 & -1.983 & 0.047 & -0.129 \\
\hline PLIW_w1 & $<-$ & Age_w1 & -0.006 & 0.003 & -1.947 & 0.052 & -0.134 \\
\hline BoundaryOrg1_w1 & $<-$ & BoundaryOrg & 1 & & & & 0.914 \\
\hline BoundaryOrg2_w1 & $<-$ & BoundaryOrg & 0.946 & 0.034 & 27.88 & $* * *$ & 0.924 \\
\hline BoundaryOrg3_w1 & $<-$ & BoundaryOrg & 0.932 & 0.033 & 28.227 & $* * *$ & 0.929 \\
\hline BoundaryOrg4_w1 & $<-$ & BoundaryOrg & 0.898 & 0.037 & 24.352 & $* * *$ & 0.879 \\
\hline BoundaryPref1_w1 & $<-$ & BoundaryPref & 1 & & & & 0.909 \\
\hline BoundaryPref2_w1 & $<-$ & BoundaryPref & 0.937 & 0.039 & 24.293 & $* * *$ & 0.9 \\
\hline BoundaryPref3_w1 & $<-$ & BoundaryPref & 0.857 & 0.04 & 21.629 & $* * *$ & 0.854 \\
\hline BoundaryPref4_w1 & $<-$ & BoundaryPref & 0.823 & 0.039 & 20.905 & $* * *$ & 0.84 \\
\hline BoundaryOrg & $<-$ & Congruence & -0.5 & & & & -0.607 \\
\hline BoundaryPref & $<-$ & Congruence & 0.5 & & & & 0.693 \\
\hline Enhance_NtW_Val_w1 & $<-$ & Congruence & 0.003 & 0.014 & 0.24 & 0.81 & 0.014 \\
\hline PCS_w2 & $<-$ & Congruence & 0.344 & 0.189 & 1.822 & 0.068 & 0.106 \\
\hline PLIW_w1 & $<-$ & Congruence & 0.013 & 0.016 & 0.784 & 0.433 & 0.05 \\
\hline Enhance_NtW_Val_w1 & $<-$ & Education_w1 & 0.055 & 0.022 & 2.568 & 0.01 & 0.154 \\
\hline PCS_w2 & $<-$ & Education_w1 & 0.558 & 0.298 & 1.876 & 0.061 & 0.111 \\
\hline PLIW_w1 & $<-$ & Education_w1 & 0.02 & 0.025 & 0.802 & 0.423 & 0.051 \\
\hline Enhance_NtW_Val1_w1 & $<-$ & Enhance_NtW_Val_w1 & 1 & & & & 0.843 \\
\hline Enhance_NtW_Val2_w1 & $<-$ & Enhance_NtW_Val_w1 & 1.086 & 0.052 & 20.739 & $* * *$ & 0.914 \\
\hline Enhance_NtW_Val3_w1 & $<-$ & Enhance_NtW_Val_w1 & 1.193 & 0.058 & 20.627 & $* * *$ & 0.91 \\
\hline PCS_w2 & $<-$ & Enhance_NtW_Val_w1 & 0.55 & 0.864 & 0.637 & 0.524 & 0.039 \\
\hline Enhance_NtW_Val_w1 & $<-$ & Income_w2 & 0.001 & 0.016 & 0.051 & 0.959 & 0.003 \\
\hline PCS_w2 & $<-$ & Income_w2 & -0.15 & 0.22 & -0.683 & 0.495 & -0.043 \\
\hline PLIW_w1 & $<-$ & Income_w2 & -0.006 & 0.019 & -0.302 & 0.763 & -0.021 \\
\hline Enhance_NtW_Val_w1 & $<-$ & KidsAdult_w1 & 0.013 & 0.068 & 0.187 & 0.852 & 0.012 \\
\hline PCS_w2 & $<-$ & KidsAdult_w1 & -0.831 & 0.928 & -0.895 & 0.371 & -0.054 \\
\hline PLIW_w1 & $<-$ & KidsAdult_w1 & 0.055 & 0.08 & 0.681 & 0.496 & 0.045 \\
\hline BoundaryOrg & $<-$ & Level & 1 & & & & 0.717 \\
\hline BoundaryPref & $<-$ & Level & 1 & & & & 0.82 \\
\hline Enhance_NtW_Val_w1 & $<-$ & Level & -0.034 & 0.024 & -1.444 & 0.149 & -0.086 \\
\hline PCS_w2 & $<-$ & Level & -0.555 & 0.323 & -1.72 & 0.085 & -0.101 \\
\hline PLIW_w1 & $<-$ & Level & -0.011 & 0.028 & -0.379 & 0.705 & -0.024 \\
\hline PCS_w2 & $<-$ & PLIW_w1 & -0.268 & 0.759 & -0.353 & 0.724 & -0.021 \\
\hline PLIW1_w1 & $<-$ & PLIW_w1 & 1 & & & & 0.646 \\
\hline PLIW2_w1 & $<-$ & PLIW_w1 & 1.146 & 0.096 & 11.996 & $* * *$ & 0.845 \\
\hline PLIW3_w1 & $<-$ & PLIW_w1 & 0.954 & 0.111 & 8.615 & $* * *$ & 0.555 \\
\hline PLIW4_w1 & $<-$ & PLIW_w1 & 1.16 & 0.1 & 11.63 & $* * *$ & 0.805 \\
\hline PLIW5_w1 & $<-$ & PLIW_w1 & 0.919 & 0.114 & 8.085 & $* * *$ & 0.516 \\
\hline PLIW6_w1 & $<-$ & PLIW_w1 & 1.033 & 0.091 & 11.291 & $* * *$ & 0.773 \\
\hline
\end{tabular}




\section{Appendix H: Qualitative Code List}

\begin{tabular}{|c|c|c|}
\hline Code & Definition & Example \\
\hline \multicolumn{3}{|l|}{ Description } \\
\hline $\begin{array}{l}\text { Self- } \\
\text { Employed }\end{array}$ & $\begin{array}{l}\text { Statement that the individual is self- } \\
\text { employed or the owner of a small company. }\end{array}$ & $\begin{array}{l}\text { "I'm the owner, so I set the } \\
\text { values." }\end{array}$ \\
\hline On-Call & $\begin{array}{l}\text { Statement that the individual is required to } \\
\text { be on-call some or all of the time. }\end{array}$ & $\begin{array}{l}\text { "I work for a political campaign } \\
\text { and am on call all the time." }\end{array}$ \\
\hline Job & Statement of job or job title. & "I am a social worker" \\
\hline $\begin{array}{l}\text { Flex-place or - } \\
\text { time }\end{array}$ & $\begin{array}{l}\text { Statement that the individual participates in } \\
\text { working from home on some or all days, or } \\
\text { that there workplace offers flextime } \\
\text { policies. }\end{array}$ & $\begin{array}{l}\text { "I work from home two days per } \\
\text { week." }\end{array}$ \\
\hline \multicolumn{3}{|l|}{$\begin{array}{l}\text { Boundary } \\
\text { Management }\end{array}$} \\
\hline Integration & $\begin{array}{l}\text { Examples of how individuals enjoy } \\
\text { integration }\end{array}$ & $\begin{array}{l}\text { "I sometimes enjoy working } \\
\text { through work problems at home, } \\
\text { usually on the computer." }\end{array}$ \\
\hline Segmentation & $\begin{array}{l}\text { Examples of how individuals enjoy } \\
\text { segmentation. }\end{array}$ & $\begin{array}{l}\text { "This is a bit tricky....as what I } \\
\text { 'like' is quite different from what } \\
\text { happens. I would certainly prefer } \\
\text { to leave work behind, but it does } \\
\text { not happen. }\end{array}$ \\
\hline \multicolumn{2}{|l|}{ Positive } & \\
\hline $\begin{array}{l}\text { Positive } \\
\text { Culture }\end{array}$ & $\begin{array}{l}\text { Description of how the organization } \\
\text { supports work-nonwork balance and } \\
\text { encourages employee efforts to maintain } \\
\text { balance. }\end{array}$ & $\begin{array}{l}\text { "Work/Life balance at my } \\
\text { company is stated as a top } \\
\text { priority, and the company } \\
\text { generally delivers in that regard." }\end{array}$ \\
\hline Enhancement & $\begin{array}{l}\text { Examples of work-nonwork enhancement, } \\
\text { in either direction. }\end{array}$ & $\begin{array}{l}\text { "I used to be very shy, but going } \\
\text { into a career that involved } \\
\text { interfacing with the public built } \\
\text { my interpersonal skills and } \\
\text { convinced me I was actually good } \\
\text { with people. Also, being a parent } \\
\text { has proven to be good practice for } \\
\text { being a supervisor/manager." }\end{array}$ \\
\hline Good Fit & $\begin{array}{l}\text { Examples of a positive fit between } \\
\text { boundary preferences and boundary } \\
\text { supplies. }\end{array}$ & $\begin{array}{l}\text { "My position is flexible and able } \\
\text { to be performed remotely - so I } \\
\text { can do work from a hospital room } \\
\text { if I need to - fits my needs } \\
\text { perfectly. I can still go to the track } \\
\text { meet and get my work done. Also } \\
\text { I am devoted to children and it is a }\end{array}$ \\
\hline
\end{tabular}


child care company - great fit!"

\section{Negative \\ Examples}

Poor Culture Description of how the organization does not support work-nonwork balance, or where the practices do not support stated policies to support balance.

Conflict

Poor Fit

Family-

Related Issues

Non-Family

Related Issues

Examples of nonwork home stressors related to issues other than family.

\section{It Depends on...}

My

Occupation/

Job/ Etc.

Individuals

\section{Alternative Perspectives}

Work is a Respite
A description of how work-nonwork balance and/or boundary management practices vary by the particular occupation, organization, department, or position.

A statement that work-nonwork balance and/or boundary management practices are not determined by the organization, but rather, but other individuals.

Illustration of how work is not a demand, but actually a respite of the demands of home life.
"The organization may model one thing and exercise another. Yes, they promote good health practices and then under staff the positions causing stress and work overload."

"My work causes me to make sacrifices to my personal life."

"It is encouraged to monitor email at home, which I don't like to do."

"Having a new child makes work even more stressful. It would be wonderful to get more than 1 week off of work to properly balance my home life and work life."

"In the last month I just bought a house so my home life has taken up an extraordinary amount of time."

"I have the benefit of working an entry level job, so have less responsibility to bleed into home life. I know my coworkers and supervisors frequently respond to email from home and work on projects at home as well."

"My immediate supervisor is very supportive and allows freedom and flexibility. It is not the policy of the organization."

"Currently outside work (personal life) is more challenging than work life. Work can be a nice, independent break from home life." 
Contrasting

Domains

\section{Individual \\ Differences}

Boundaries are a Choice

Boundaries are Learned

Conscious Decisions

\section{Challenges to Balance}

Intense

Demands

Commute

Travel

Technology
A description of how pleasant experiences in one domain may actually decrease happiness in the other domain.
"The better my life is outside of work, the more I realize how much I hate my job."
A position that boundaries are a personal choice, and depend on internal variables rather than external variables such as organizational policies.

A position that boundary management strategies can be learned and acquired over time, rather than being static.

Description of conscious decisions or efforts the individual makes to maintain proper work-nonwork balance.

"These are all personal choices, it's not up to my employer or work to create balance for me. I am an adult and make my own choices."

"It took several years and being put in antidepressants twice before I learned how to mostly balance my work and home life. It is a skill that too few people have learned and far too many companies don't value employees who separate work and home life."

"I feel so strongly about a balance of work and home life, that I have turned down a promotion because I would have been expected to work more hours."

Times of high stress or high demands at work, especially with too little resources, make work-nonwork balance especially difficult.

"Extremely heavy workloads frequently require me to work on my days off. There is no light at the end of the tunnel. Stresses caused by work also negatively impact my home life."

"My awful commute is more to blame for my craziness (too tired when I come home) fighting traffic both ways."

"I travel extensively and I find it more difficult to balance work/family when I am in a different time zone."

"Because of e-mail and texting, work is far more intrusive than it was, say, 15 years ago. It is much more difficult to truly leave work at work." 
Tired/ Description that the main health complaint

Exhausted

\section{Timing}

Calendar Year

Unusual

Circumstances is exhaustion, feeling tired, lack of sleep, or lack of leisure time.

Indications that the time of year the survey was administered may not be reflective of usual times.

Description of unusual circumstances that may have influenced their health or work status.
"My leisure time is so rare that it is usually spent sleeping/relaxing."

"Just a reminder that some of your respondents may have spent the past month or a good part of it on vacation."

"I have been pregnant (just gave birth this week)"

\section{Survey}

\section{Construction}

Feedback

Feedback can include suggestions for other topics that should be included, or comments on aspects of the survey the respondent did or did not like.
"I think asking about exercise habits would lend more information about frame of mind. People that exercise, in my opinion, are happier and more balanced." 


\section{Appendix I: Simplified LCM without Mediator Results}

Physical Health

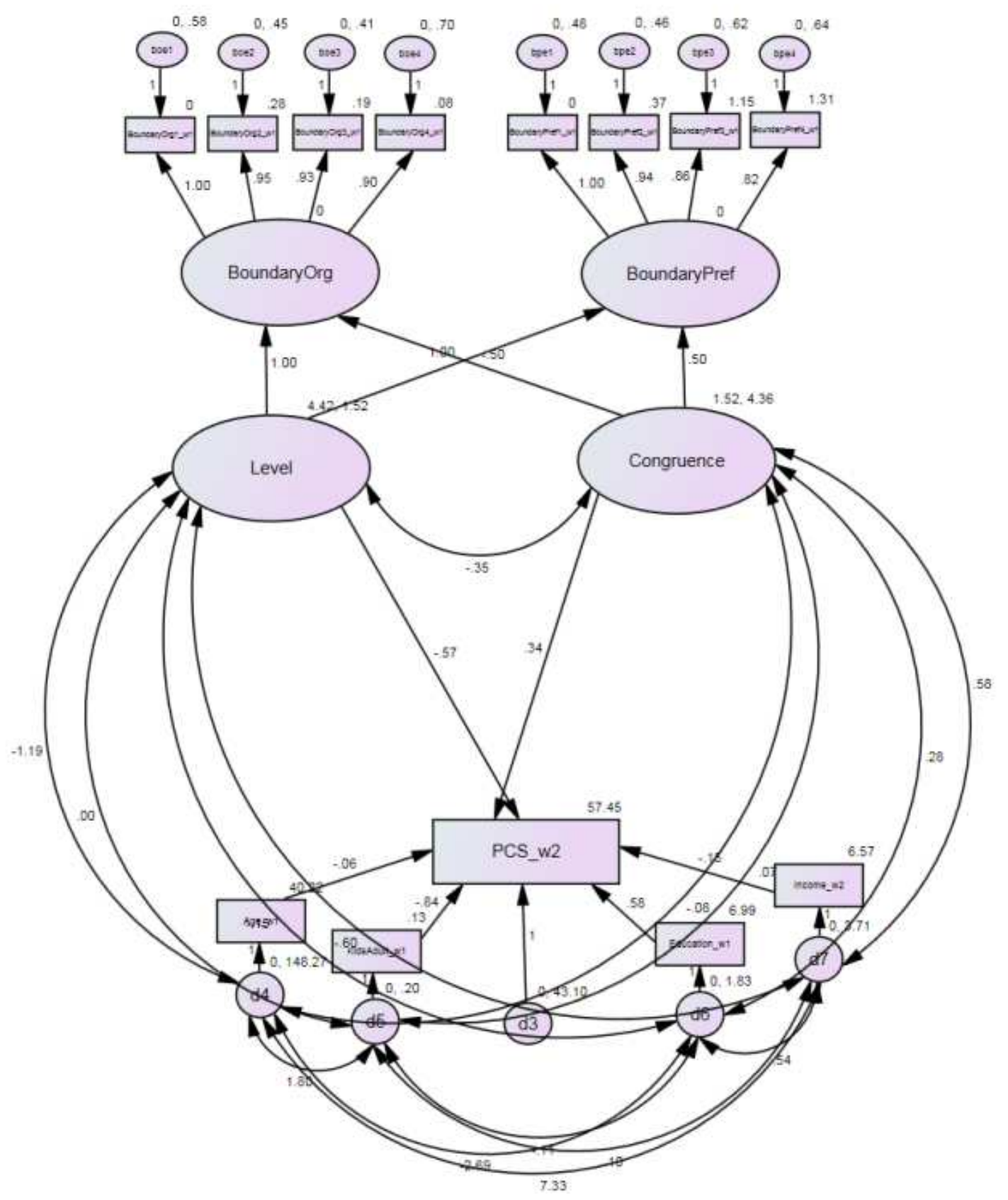

Fit Statistics:

- $\chi^{2}(49)=94.34, p<.001$

- CFI: .982

- RMSEA: .054 
$\underline{\text { Physical Health Output }}$

\begin{tabular}{lclrrrrr}
\hline & & & Est. & S.E. & C.R. & p & Std. Est. \\
\hline PCS_w2 & $<--$ & Age_w1 & -0.063 & 0.035 & -1.809 & 0.070 & -0.113 \\
BoundaryOrg1_w1 & $<--$ & BoundaryOrg & 1 & & & & 0.914 \\
BoundaryOrg2_w1 & $<--$ & BoundaryOrg & 0.947 & 0.034 & 27.888 & $* * *$ & 0.925 \\
BoundaryOrg3_w1 & $<--$ & BoundaryOrg & 0.932 & 0.033 & 28.210 & $* * *$ & 0.929 \\
BoundaryOrg4_w1 & $<--$ & BoundaryOrg & 0.898 & 0.037 & 24.309 & $* * *$ & 0.879 \\
BoundaryPref1_w1 & $<--$ & BoundaryPref & 1 & & & & 0.909 \\
BoundaryPref2_w1 & $<--$ & BoundaryPref & 0.938 & 0.039 & 24.275 & $* * *$ & 0.901 \\
BoundaryPref3_w1 & $<--$ & BoundaryPref & 0.858 & 0.040 & 21.620 & $* * *$ & 0.854 \\
BoundaryPref4_w1 & $<--$ & BoundaryPref & 0.824 & 0.039 & 20.907 & $* * *$ & 0.840 \\
BoundaryOrg & $<--$ & Congruence & -0.500 & & & & -0.607 \\
BoundaryPref & $<--$ & Congruence & 0.500 & & & & 0.693 \\
PCS_w2 & $<--$ & Congruence & 0.344 & 0.189 & 1.822 & 0.069 & 0.106 \\
PCS_w2 & $<--$ & Education_w1 & 0.584 & 0.293 & 1.990 & 0.047 & 0.116 \\
PCS_w2 & $<--$ & Income_w2 & -0.149 & 0.220 & -0.678 & 0.498 & -0.042 \\
PCS_w2 & $<--$ & KidsAdult_w1 & -0.841 & 0.928 & -0.906 & 0.365 & -0.055 \\
BoundaryOrg & $<--$ & Level & 1 & & & & 0.717 \\
BoundaryPref & $<--$ & Level & 1 & & & & 0.820 \\
PCS_w2 & $<--$ & Level & -0.572 & 0.322 & -1.780 & 0.075 & -0.104 \\
\hline
\end{tabular}




\section{Mental Health}

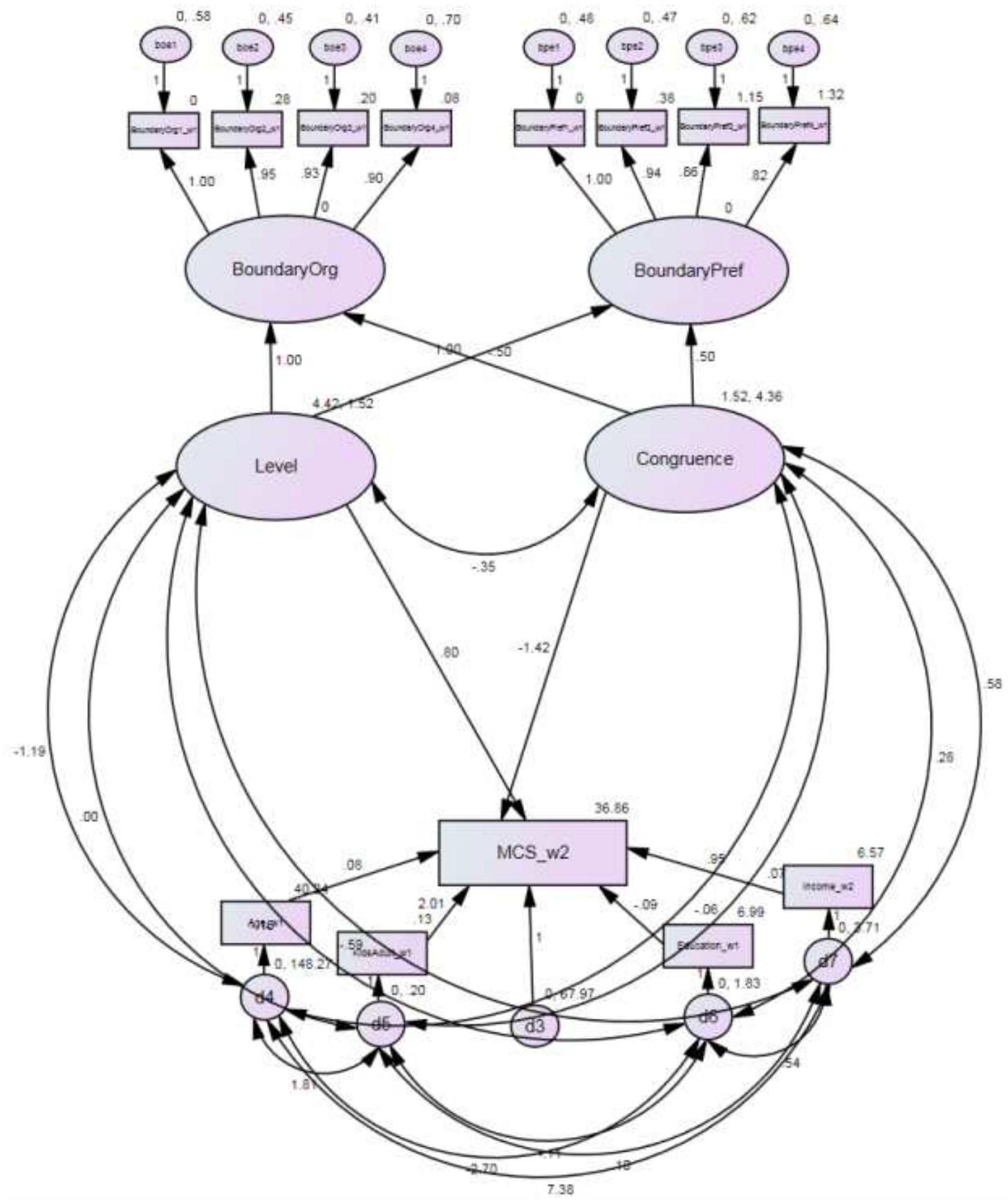

Fit Statistics:

- $\chi^{2}(49)=85.94, p>.001$

- CFI: .985

- RMSEA: 049 
Mental Health Output

\begin{tabular}{lclrrrrr}
\hline & & & Est. & S.E. & C.R. & P & Std. Est. \\
\hline MCS_w2 & $<--$ & Age_w1 & 0.076 & 0.044 & 1.720 & 0.085 & 0.102 \\
BoundaryOrg1_w1 & $<--$ & BoundaryOrg & 1 & & & & 0.915 \\
BoundaryOrg2_w1 & $<--$ & BoundaryOrg & 0.945 & 0.034 & 27.969 & $* * *$ & 0.924 \\
BoundaryOrg3_w1 & $<--$ & BoundaryOrg & 0.931 & 0.033 & 28.273 & $* * *$ & 0.928 \\
BoundaryOrg4_w1 & $<--$ & BoundaryOrg & 0.897 & 0.037 & 24.362 & $* * *$ & 0.879 \\
BoundaryPref1_w1 & $<--$ & BoundaryPref & 1 & & & & 0.909 \\
BoundaryPref2_w1 & $<--$ & BoundaryPref & 0.936 & 0.039 & 24.287 & $* * *$ & 0.900 \\
BoundaryPref3_w1 & $<--$ & BoundaryPref & 0.857 & 0.040 & 21.658 & $* * *$ & 0.854 \\
BoundaryPref4_w1 & $<--$ & BoundaryPref & 0.823 & 0.039 & 20.939 & $* * *$ & 0.840 \\
BoundaryOrg & $<--$ & Congruence & -0.500 & & & & -0.607 \\
BoundaryPref & $<--$ & Congruence & 0.500 & & & & 0.694 \\
MCS_w2 & $<--$ & Congruence & -1.424 & 0.239 & -5.958 & $* * *$ & -0.327 \\
MCS_w2 & $<--$ & Education_w1 & -0.089 & 0.370 & -0.241 & 0.809 & -0.013 \\
MCS_w2 & $<--$ & Income_w2 & 0.952 & 0.278 & 3.428 & $* * *$ & 0.202 \\
MCS_w2 & $<--$ & KidsAdult_w1 & 2.010 & 1.170 & 1.717 & 0.086 & 0.098 \\
BoundaryOrg & $<--$ & Level & 1 & & & & 0.717 \\
BoundaryPref & $<--$ & Level & 1 & & & & 0.820 \\
MCS_w2 & $<--$ & Level & 0.799 & 0.408 & 1.960 & 0.050 & 0.109 \\
\hline
\end{tabular}




\section{Appendix J: Significance Comparisons between Time 1 and Time 2 Mediator}

\section{Models}

\begin{tabular}{|c|c|c|c|c|}
\hline \multirow[t]{2}{*}{ Model } & \multicolumn{2}{|c|}{ Pathway } & \multicolumn{2}{|c|}{ Significance } \\
\hline & Predictor & Outcome & Using Time 1 Mediators & Using Time 2 Mediators \\
\hline \multirow[t]{5}{*}{1} & Congruence & WtN Beh & - & - \\
\hline & Congruence & PLIW & $n s$ & $n s$ \\
\hline & Congruence & MCS & - & - \\
\hline & WtN Beh & MCS & $n s$ & $n s$ \\
\hline & PLIW & MCS & - & - \\
\hline \multirow[t]{5}{*}{2} & Congruence & WtN Beh & - & - \\
\hline & Congruence & PLIW & $n s$ & $n s$ \\
\hline & Congruence & PCS & + & + \\
\hline & WtN Beh & PCS & $n s$ & + \\
\hline & PLIW & PCS & $n s$ & $n s$ \\
\hline \multirow[t]{5}{*}{3} & Congruence & WtN Beh & - & - \\
\hline & Congruence & WIPL & + & + \\
\hline & Congruence & PCS & + & + \\
\hline & WtN Beh & PCS & $n s$ & $n s$ \\
\hline & WIPL & PCS & $n s$ & - \\
\hline \multirow[t]{5}{*}{4} & Congruence & WtN Beh & - & - \\
\hline & Congruence & WIPL & + & + \\
\hline & Congruence & MCS & - & - \\
\hline & WtN Beh & MCS & $n s$ & $n s$ \\
\hline & WIPL & MCS & - & - \\
\hline \multirow[t]{5}{*}{5} & Congruence & WtN Aff & $n s$ & $n s$ \\
\hline & Congruence & WIPL & + & + \\
\hline & Congruence & MCS & - & - \\
\hline & WtN Aff & MCS & $n s$ & $n s$ \\
\hline & WIPL & MCS & - & - \\
\hline \multirow[t]{5}{*}{6} & Congruence & WtN Aff & $n s$ & $n s$ \\
\hline & Congruence & WIPL & + & + \\
\hline & Congruence & PCS & + & + \\
\hline & WtN Aff & PCS & + & + \\
\hline & WIPL & PCS & $n s$ & - \\
\hline \multirow[t]{5}{*}{7} & Congruence & WtN Aff & $n s$ & $n s$ \\
\hline & Congruence & PLIW & $n s$ & $n s$ \\
\hline & Congruence & PCS & + & + \\
\hline & WtN Aff & PCS & + & + \\
\hline & PLIW & PCS & $n s$ & $n s$ \\
\hline 8 & Congruence & WtN Aff & $n s$ & $n s$ \\
\hline
\end{tabular}




\begin{tabular}{|c|c|c|c|c|}
\hline & Congruence & PLIW & $n s$ & $n s$ \\
\hline & Congruence & MCS & - & - \\
\hline & WtN Aff & MCS & $n s$ & $n s$ \\
\hline & PLIW & MCS & - & - \\
\hline \multirow[t]{5}{*}{9} & Congruence & WtN Val & - & - \\
\hline & Congruence & PLIW & $n s$ & $n s$ \\
\hline & Congruence & MCS & - & - \\
\hline & WtN Val & MCS & $n s$ & + \\
\hline & PLIW & MCS & - & - \\
\hline \multirow[t]{5}{*}{10} & Congruence & WtN Val & - & - \\
\hline & Congruence & PLIW & $n s$ & $n s$ \\
\hline & Congruence & PCS & + & + \\
\hline & WtN Val & PCS & $n s$ & $n s$ \\
\hline & PLIW & PCS & $n s$ & $n s$ \\
\hline \multirow[t]{5}{*}{11} & Congruence & WtN Val & - & - \\
\hline & Congruence & WIPL & + & + \\
\hline & Congruence & PCS & + & + \\
\hline & WtN Val & PCS & $n s$ & $n s$ \\
\hline & WIPL & PCS & $n s$ & - \\
\hline \multirow[t]{5}{*}{12} & Congruence & WtN Val & - & - \\
\hline & Congruence & WIPL & + & + \\
\hline & Congruence & MCS & - & - \\
\hline & WIPL & MCS & - & - \\
\hline & WtN Val & MCS & $n s$ & $n s$ \\
\hline \multirow[t]{5}{*}{13} & Congruence & NtW Beh & $n s$ & $n s$ \\
\hline & Congruence & WIPL & + & + \\
\hline & Congruence & MCS & - & - \\
\hline & NtW Beh & MCS & $n s$ & $n s$ \\
\hline & WIPL & MCS & - & - \\
\hline \multirow[t]{5}{*}{14} & Congruence & NtW Beh & $n s$ & $n s$ \\
\hline & Congruence & WIPL & + & + \\
\hline & Congruence & PCS & + & + \\
\hline & NtW Beh & PCS & $n s$ & $n s$ \\
\hline & WIPL & PCS & $n s$ & - \\
\hline \multirow[t]{5}{*}{15} & Congruence & NtW Beh & $n s$ & $n s$ \\
\hline & Congruence & PLIW & $n s$ & $n s$ \\
\hline & Congruence & PCS & + & + \\
\hline & NtW Beh & PCS & $n s$ & $n s$ \\
\hline & PLIW & PCS & $n s$ & $n s$ \\
\hline \multirow[t]{4}{*}{16} & Congruence & NtW Beh & $n s$ & $n s$ \\
\hline & Congruence & PLIW & $n s$ & $n s$ \\
\hline & Congruence & MCS & - & - \\
\hline & NtW Beh & MCS & $n s$ & $n s$ \\
\hline
\end{tabular}


PLIW MCS

17 Congruence

Congruence

Congruence

NtW Aff

PLIW

$\mathrm{NtW}$ Aff

PLIW

PCS

PCS

PCS

18 Congruence

Congruence

Congruence

NtW Aff

PLIW

NtW Aff

PLIW

MCS

MCS

MCS

19 Congruence

Congruence

Congruence

NtW Aff

WIPL

NtW Aff

WIPL

MCS

MCS

MCS

20 Congruence

Congruence

Congruence

NtW Aff

WIPL

$\mathrm{NtW}$ Aff

WIPL

PCS

PCS

PCS

$\begin{array}{cc}n s & - \\ n s & n s \\ + & + \\ + & + \\ n s & n s\end{array}$

21 Congruence

Congruence

$\mathrm{NtW}$ Val

WIPL

Congruence

PCS

$\mathrm{NtW}$ Val

PCS

WIPL

PCS

$\begin{array}{cc}n s & - \\ n s & n s \\ - & - \\ n s & + \\ - & -\end{array}$

22 Congruence

Congruence

Congruence

NtW Val

WIPL

MCS

$\mathrm{NtW}$ Val

MCS

WIPL

MCS

23 Congruence

Congruence

$\mathrm{NtW}$ Val

ns

$-$

$+$

$-$

ns

ns

$-$

Congruence

PLIW

$\mathrm{NtW}$ Val

MCS

PLIW

MCS

MCS

24 Congruence

$\mathrm{NtW}$ Val

Congruence

PLIW

Congruence

PCS

$\mathrm{NtW}$ Val

PCS

PLIW

PCS

$\begin{array}{cc}n s & - \\ + & + \\ + & + \\ + & n s \\ n s & n s\end{array}$

ns ns

$+\quad+$

$+\quad+$

ns +

ns $\quad-$

ns ns

$+\quad+$

-

ns ns

-

ns $n s$

ns ns

-

ns ns

-

ns $n s$

$n s \quad n s$

ns ns

ns $n s$

$n s \quad n s$


Note. A " + " indicates a significant positive relationship between the predictor and the outcome at the $\mathrm{p}<$ .05 level. A "-" indicates a significant negative relationship between the predictor and the outcome at the $\mathrm{p}$ $<.05$ level. An "ns" indicates no significant relationship between the predictor and the outcome.

Appendix K. Supplementary Analyses for Non-Significant Polynomial Regression

\section{Models}

\section{Nonwork-to-Work Conflict}

Response Surface Map of Congruence \& Nonwork-to-Work Conflict

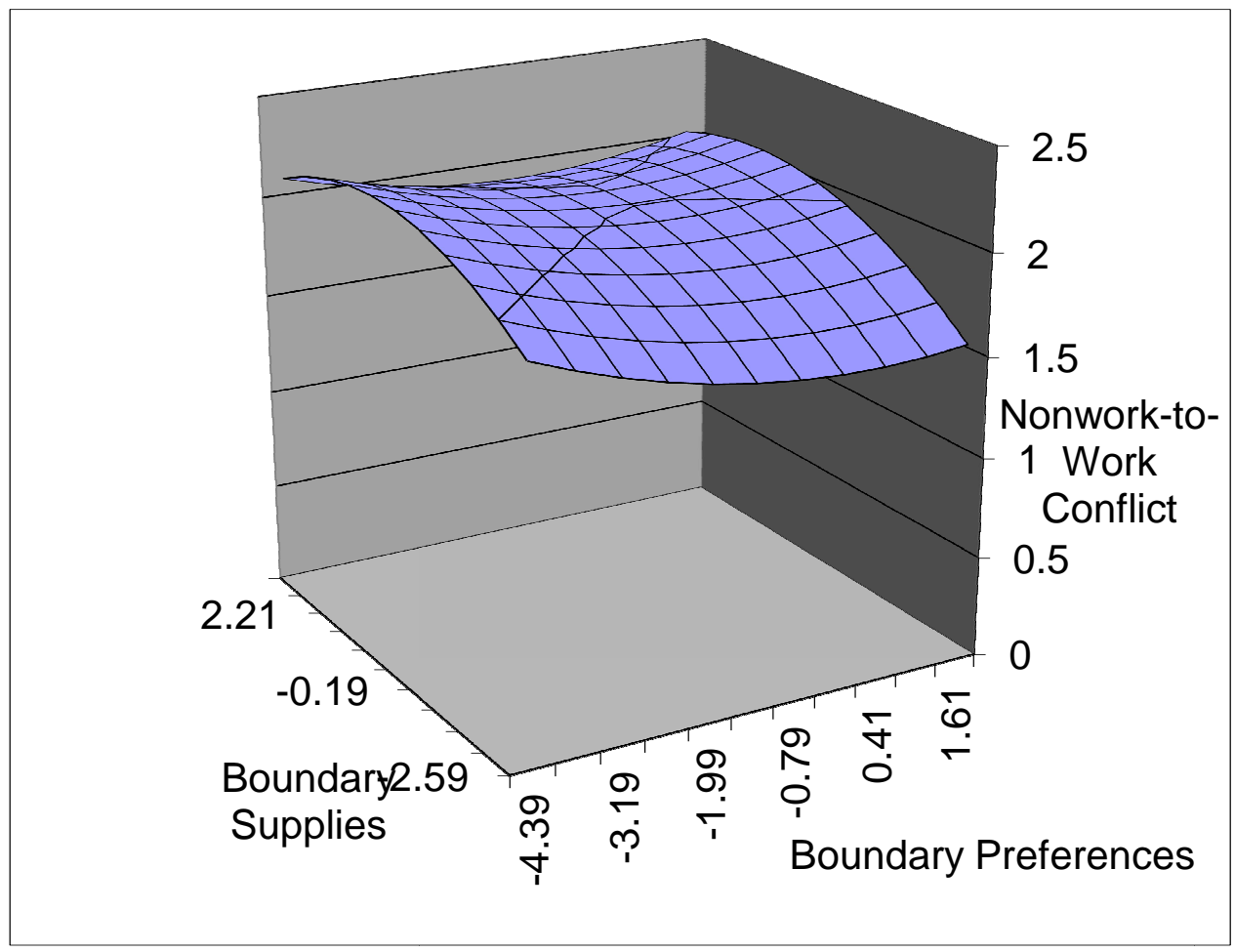

$P=S$ and $P=-S$ Slopes for Congruence \& Nonwork-to-Work Conflict 

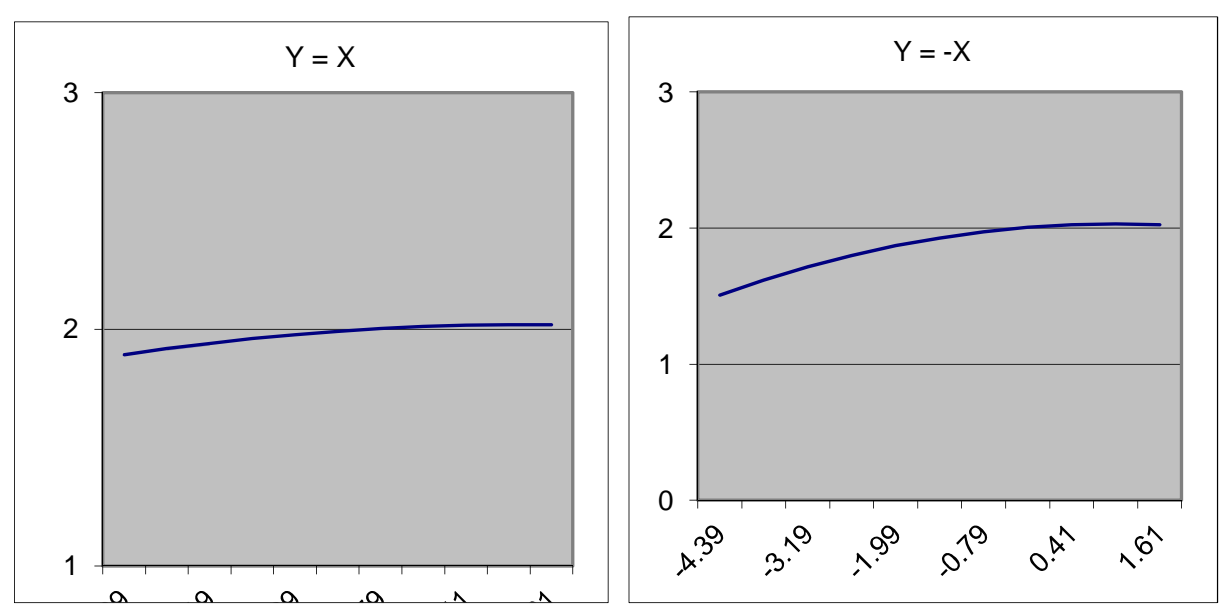

For the outcome of nonwork-to-work conflict, both coefficients along the $P=S$ line were nonsignificant, indicating that all instances of perfect fit have a comparable influence on nonwork-to-work conflict, regardless of where that "perfect fit" falls on the continuum.

In this case, significance testing of the polynomial regression coefficients indicated that neither of the coefficients along the $P=-S$ line were statistically significant. This indicates that certain types of misfit are more or less "bad" for nonwork-to-work conflict than others. Specifically, instances of low-preferences/highsupplies appear to result in higher levels of nonwork-to-work conflict than highpreferences/low-supplies. 


\section{Work-to-Nonwork Affective Enhancement.}

Response Surface Map of Congruence \& Work-to-Nonwork Affective Enhancement

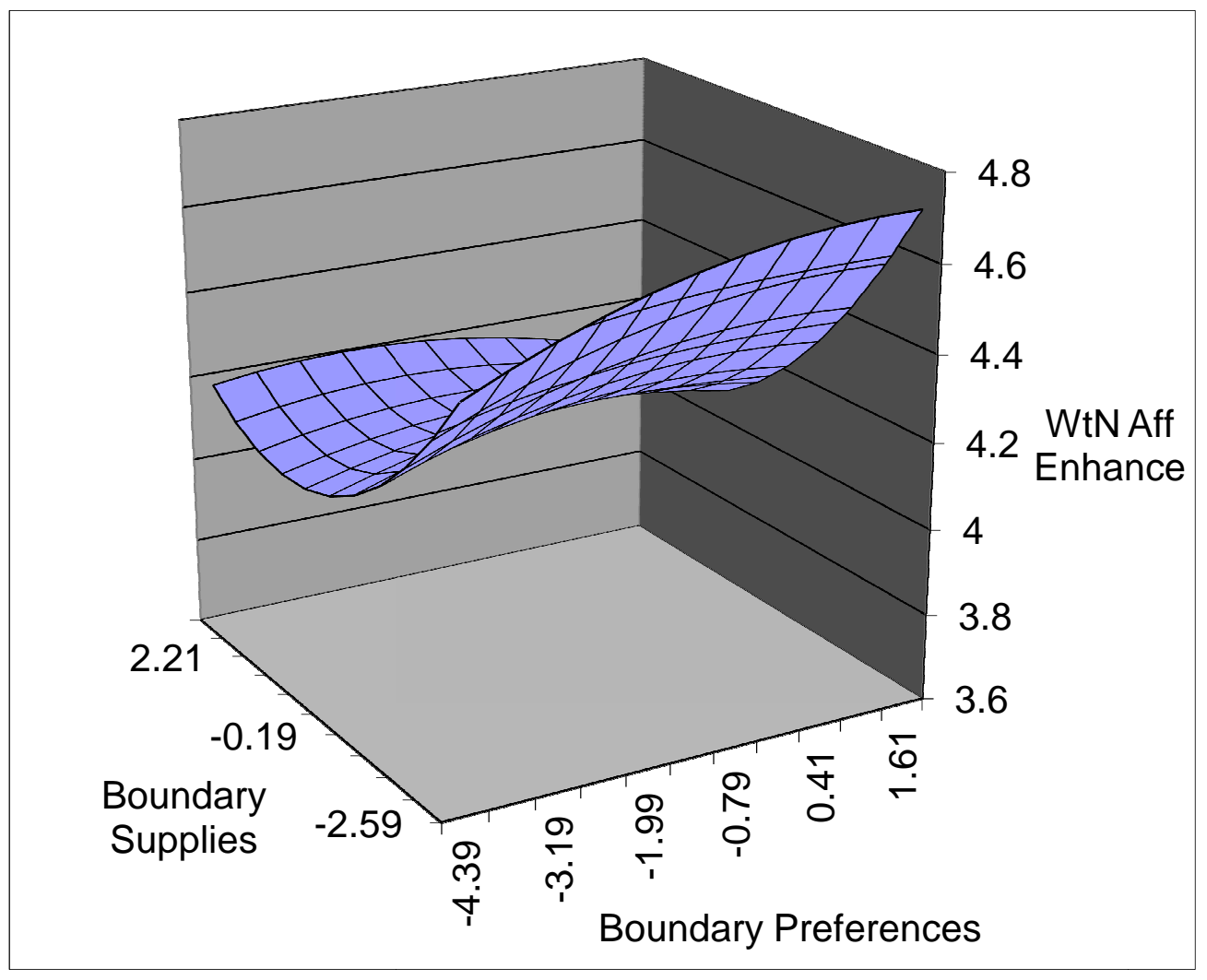

$P=S$ and $P=-S$ Slopes for Congruence \& Work-to-Nonwork Affective Enhancement
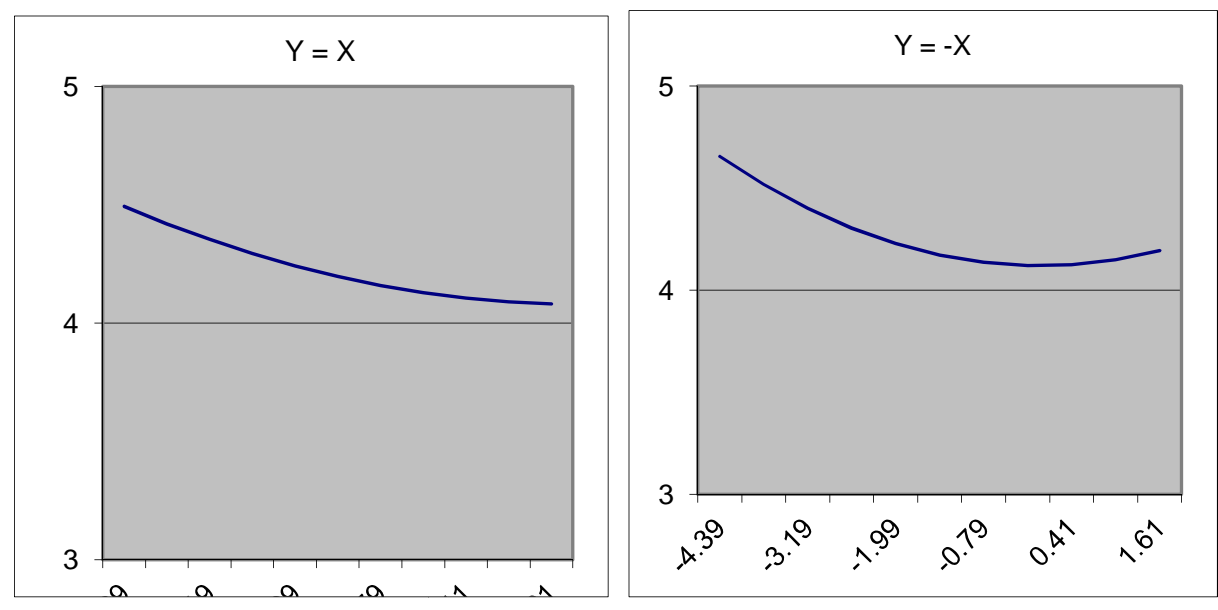
For the outcome of work-to-nonwork affective enhancement, both coefficients along the $P=S$ line were nonsignificant, indicating that all instances of perfect fit have a comparable influence on work-to-nonwork affective enhancement, regardless of where that "perfect fit" falls on the continuum.

Both coefficients along the $P=-S$ line were positive and nonsignificant. The response surface map indicates that work-to-nonwork affective enhancement levels appear to be highest in high-preferences/low-supplies misfit situations rather than lowpreferences/high-supplies. 


\section{Nonwork to Work Behavior-Based Enhancement.}

Response Surface Map of Congruence \& Nonwork-to-Work Behavior-Based Enhancement

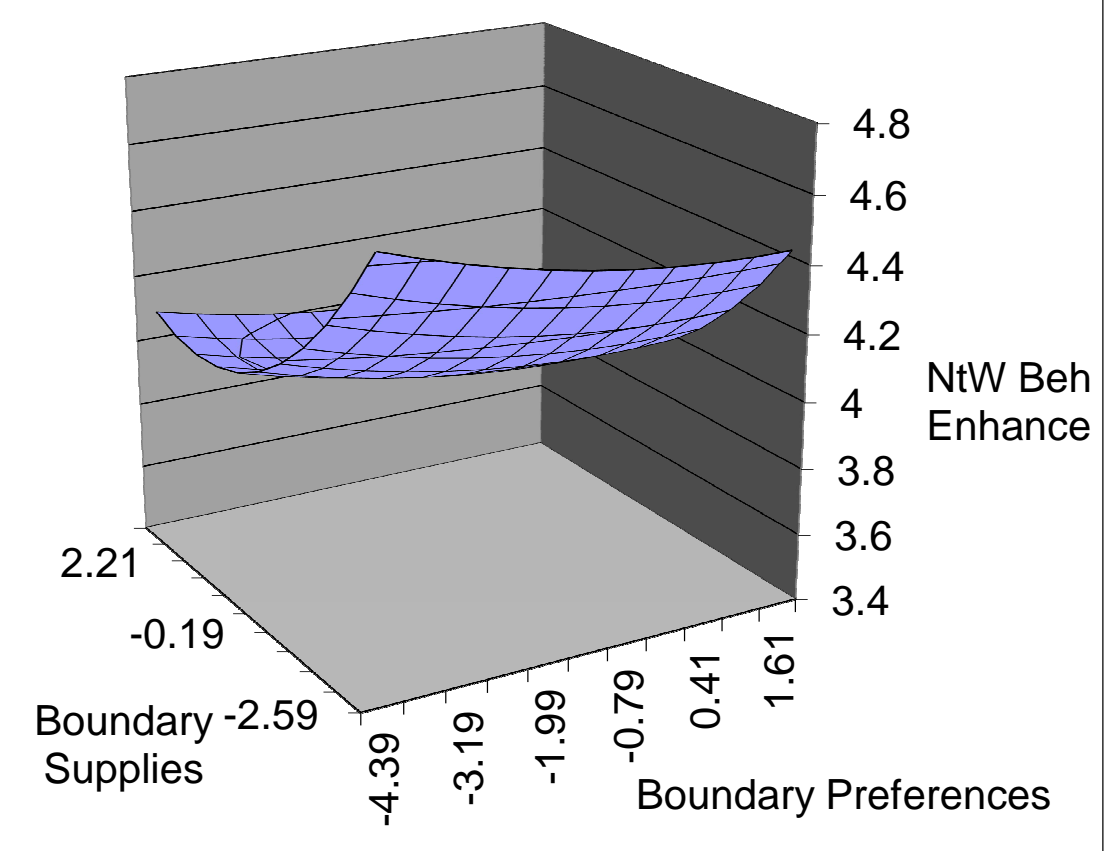

$P=S$ and $P=-S$ Slopes for Congruence \& Nonwork-to-Work Behavior-Based

Enhancement
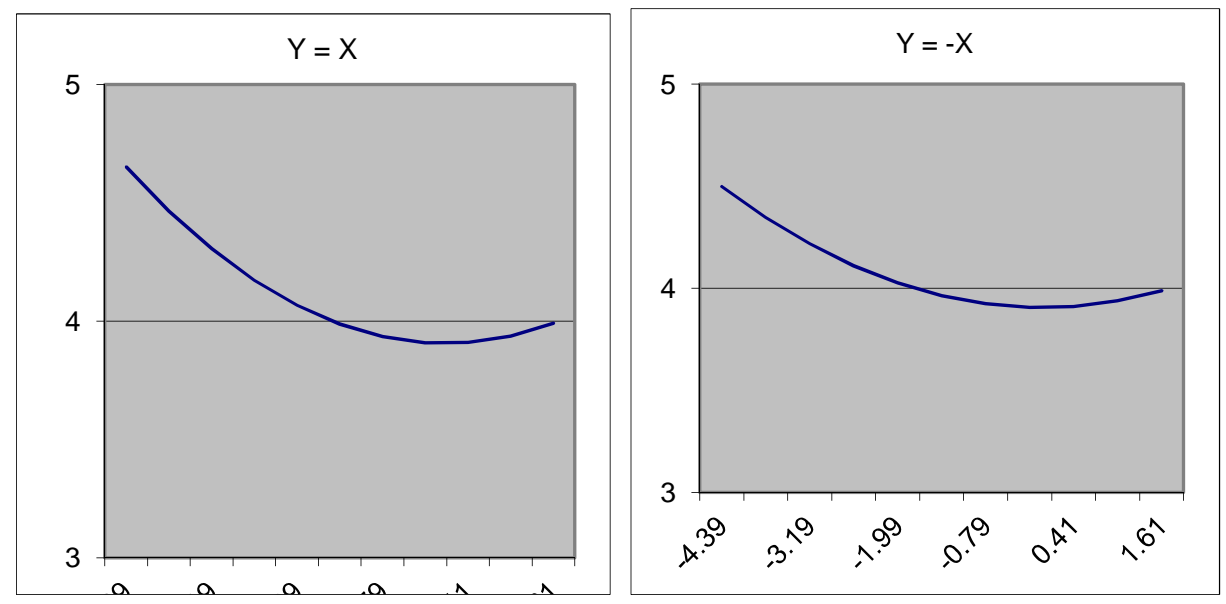
For the outcome of nonwork-to-work behavior-based enhancement, both coefficients along the $P=S$ line were nonsignificant, indicating that all instances of perfect fit have a comparable influence on nonwork-to-work behavior-based enhancement, regardless of where that "perfect fit" falls on the continuum.

Both coefficients along the $P=-S$ line were positive and nonsignificant, indicating that some instances of misfit are better for the outcome of nonwork-to-work behavior-based enhancement than others. Specifically, it appears that highpreferences/low-supplies appear to be more beneficial to this facet of enhancement than low-preferences/high-supplies. 


\section{Nonwork-to-Work Affective Enhancement.}

Response Surface Map of Congruence \& Nonwork-to-Work Affective Enhancement

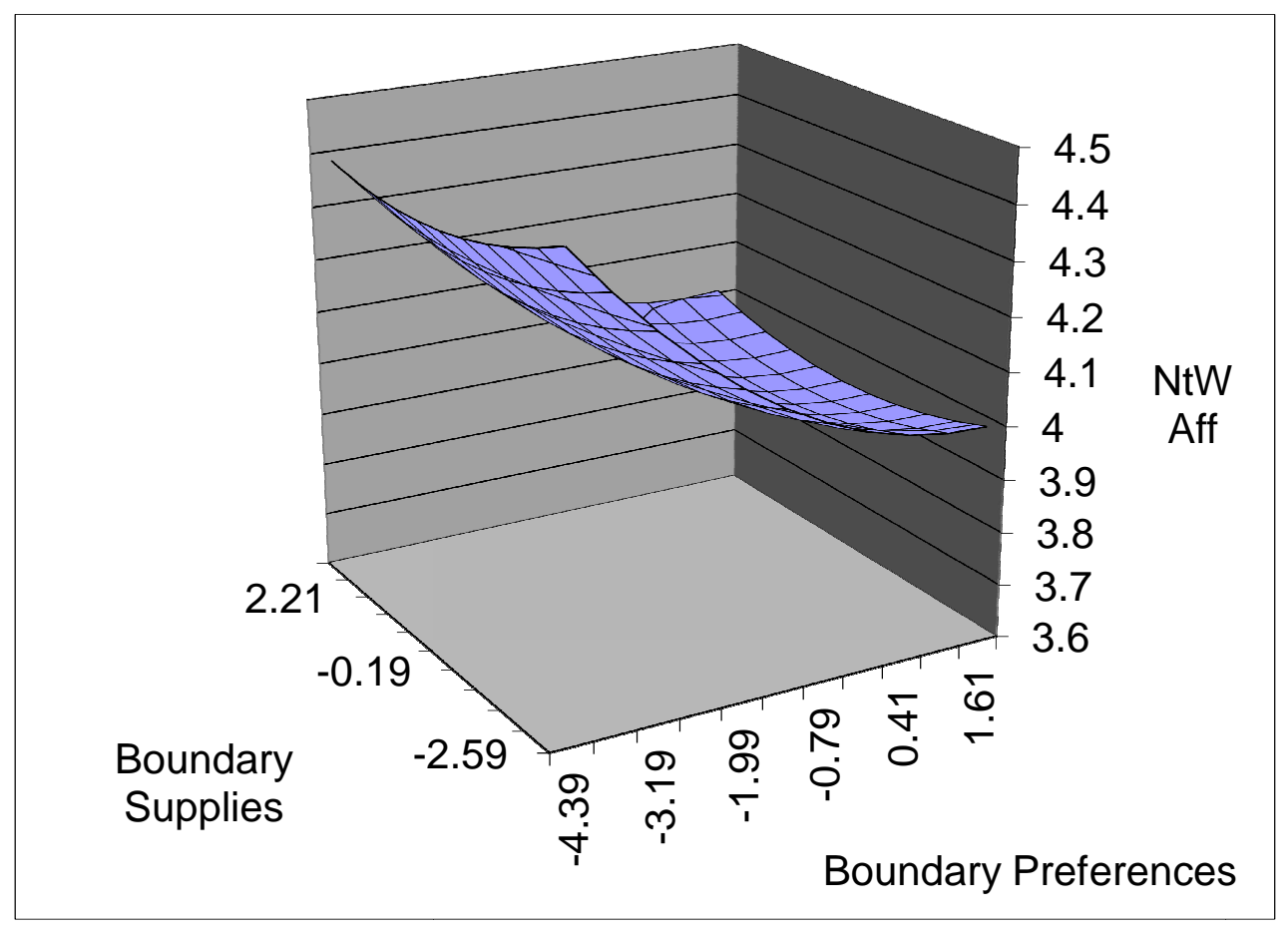

$P=S$ and $P=-S$ Slopes for Congruence \& Nonwork-to-Work Affective Enhancement
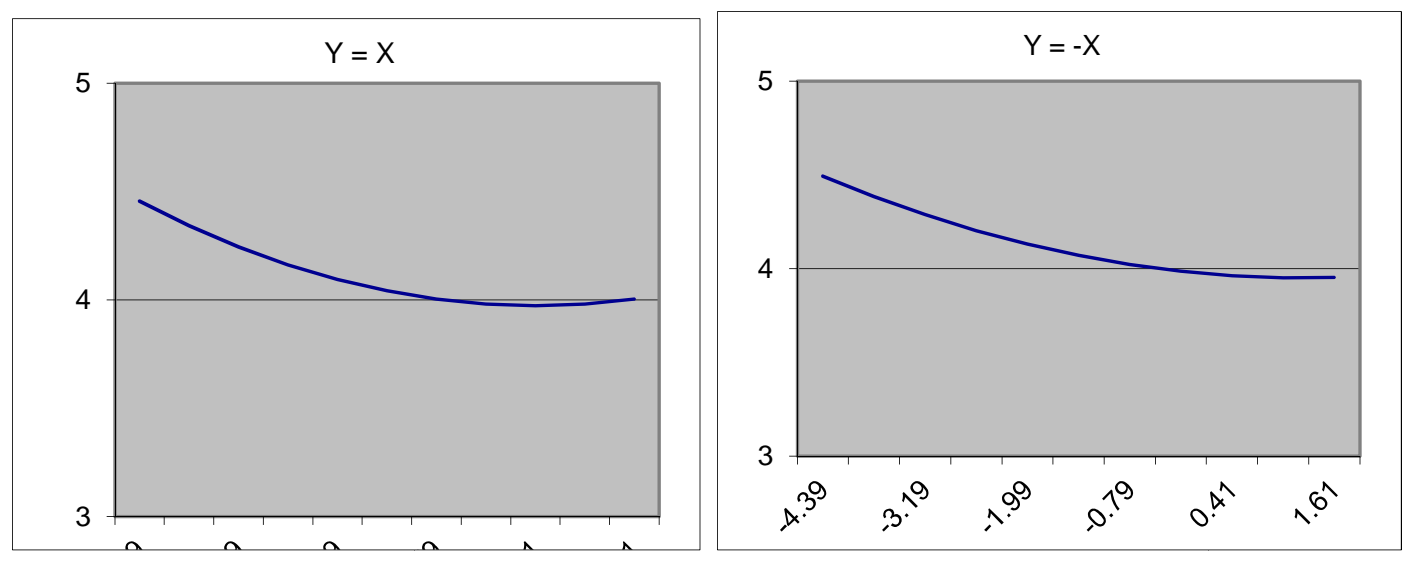
For the outcome of nonwork-to-work affective enhancement, both coefficients along the $P=S$ line were nonsignificant, indicating that all instances of perfect fit have a comparable influence on nonwork-to-work affective enhancement, regardless of where that "perfect fit" falls on the continuum.

Both coefficients along the $P=-S$ line were nonsignificant, indicating that some instances of misfit were more detrimental than others. It appears that lowpreferences/high-supplies misfit is more conducive to generating nonwork-to-work affective enhancement than high-preferences/low-supplies. 